\title{
Cellular mechanisms of odor detection in the olfactory system of the red flour beetle
}

\section{Tribolium castaneum}

\author{
Dissertation \\ for the award of the degree \\ "Doctor rerum naturalium" \\ of the Georg-August-University Göttingen \\ within the doctoral program "Sensory and Motor Neuroscience" \\ of the Georg-August-University School of Science (GAUSS)
}

submitted by

Alice Christine Montino, née Metzger

from Nürtingen a. N.

(Baden Württemberg, Germany)

Göttingen, 2015 



\section{Thesis Committee}

Prof. Dr. Ernst A. Wimmer (Supervisor, First Reviewer)

GZMB

Department of Developmental Biology

Georg-August-University, Göttingen

Prof. Dr. Martin Göpfert (Second Reviewer)

Schwann-Schleiden Research Centre

Department of Cellular Neurobiology

Georg-August-University, Göttingen

Prof. Dr. André Fiala

Schwann-Schleiden Research Centre

Department of Molecular Neurobiology of Behavior

Georg-August-University, Göttingen

\section{Further members of the Examination Board}

Prof. Dr. Gregor Bucher

GZMB

Department of Developmental Biology

Georg-August-University, Göttingen

Prof. Dr. Dr. Detlev Schild

University Medical Center Göttingen

Department of Neurophysiology and Cellular Biophysics

Georg-August-University, Göttingen

Prof. Dr. Reinhard Schuh

Max Planck Institute for Biophysical Chemistry

Dept. of Molecular Developmental Biology

Date of oral examination: August 31th 2015 

Affidavit:

Herewith I declare that I prepared this thesis on my own and with no other sources and aids than quoted.

Alice Christine Montino, née Metzger

Göttingen, June 2015 

"Ist es nicht kindisch, die Handlungen eines Insekts bis in die kleinsten Einzelheiten zu erkunden? Uns drücken viel schwerere Sorgen, als dass wir uns damit die Zeit vertreiben könnten. So lässt uns die bittere Erfahrung des Alters sprechen; so würde ich denken und meine Untersuchungen beenden, vermutete ich nicht im Wirrwarr der Beobachtungen die Aufklärung einiger der größten Probleme, die zu erörtern uns vergönnt ist. Was ist Leben?" 



\section{Content}

1 Summary 1

$\underline{2}$ Introduction 3

2.1 The peripheral olfactory system of insects $\quad 4$

2.1.1 Odorant binding proteins (OBPs) as carriers for odors in the sensillar lymph 5

$\begin{array}{ll}\text { 2.1.2 Odor detection at the OSN by odorant receptors } & 7\end{array}$

2.2 Signal transduction in OSNs $\quad 8$

$\begin{array}{ll}2.3 \text { Odor processing in the antennal lobe } & 10\end{array}$

2.4 Sensory neuron membrane proteins (SNMPs) 13

$\begin{array}{ll}\text { 2.4.1 SNMP function } & 14\end{array}$

2.4.2 SNMPs in T. castaneum 16

2.5 Aim of this thesis 18

3 Material and Methods $\quad 20$

$\begin{array}{ll}3.1 \text { Insect culture } & 20\end{array}$

$\begin{array}{ll}3.1 .1 \text { Tribolium castaneum culture } & 20\end{array}$

$\begin{array}{ll}\text { 3.1.2 Drosophila melanogaster culture } & 20\end{array}$

3.2 Molecular biology 20

3.2.1 Total RNA extraction $\quad 21$

3.2.2 cDNA synthesis 21

3.2.3 RACE (Rapid Amplification of cDNA Ends) cDNA synthesis 21

$\begin{array}{ll}3.2 .4 \text { Oligonucleotides } & 21\end{array}$

3.2.5 Polymerase chain reaction (PCR) 24

3.2.6 Cloning 24

3.2.7 DNA isolation of recombinant plasmids 25

3.2.8 Double-stranded RNA (dsRNA) synthesis 25

3.3 Sequence analysis and bioinformatics 26

$\begin{array}{ll}\text { 3.3.1 DNA sequence analysis } & 26\end{array}$

$\begin{array}{ll}\text { 3.3.2 Sequence alignments } & 26\end{array}$

$\begin{array}{ll}\text { 3.3.3 Phylogenetic calculations } & 26\end{array}$

$\begin{array}{ll}\text { 3.3.4 Statistical analysis } & 27\end{array}$

$\begin{array}{ll}3.4 \text { Histology } & 27\end{array}$

$\begin{array}{ll}3.4 .1 \text { Probe synthesis } & 27\end{array}$

$\begin{array}{ll}3.4 .2 \text { Probe fragmentation } & 28\end{array}$ 
3.4.3 Fluorescence in situ hybridization (FISH)

3.5 Genetics 29

$\begin{array}{ll}3.5 .1 \text { RNA interference (RNAi) } & 29\end{array}$

3.5.2 D. melanogaster transgenesis 30

3.6 Imaging 33

3.6.1 in vivo two-photon calcium imaging of D. melanogaster 33

3.6.2 Microscopy and image processing 34

3.7 Electroantennography (EAG) of T. castaneum 34

4 Results $\quad 36$

4.1 Sequence analysis of TCSNMPs 36

4.1.1 cDNA isolation and characterization of TcSNMPs 36

4.1.2 Analysis of the amino acid sequence and topology of TcSNMP proteins $\quad 40$

4.1.3 Phylogenetic analysis of TcSNMP proteins with SNMP proteins and other members of the CD36 protein family 42

4.2 Localization of SNMP expressing cells 46

4.2.1 Establishment of a FISH protocol for T. castaneum antennae 46

4.2.2 Establishment of a double FISH protocol for T. castaneum antennae 49

4.2.3 Visualization and characterization of SNMP expressing cells 51

4.2.4 Cellular organization of SNMP expressing cells within one segment 62

4.3 Examining the role of TcSNMPs in Tribolium castaneum 65

4.3.1 Examining the role of neuronal TcSNMPs in pheromone and beetle odor detection 66

4.3.2 Examining the role of neuronal TcSNMPs for the detection of fatty acid $\begin{array}{ll}\text { derivatives } & 67\end{array}$

4.3.3 Validation of the RNAi-induced knockdown by FISH 71

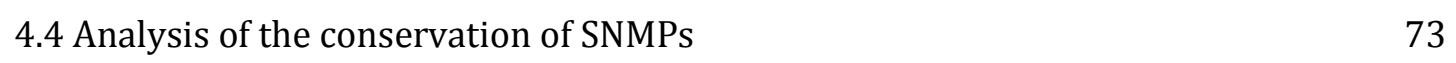

4.4.1 TcSNMPs do not rescue DmSNMP1 loss-of-function in D. melanogaster $\quad 76$

4.4.2 TcSNMPs modify the odor response profile of the DA1 glomerulus of

D. melanogaster 78

4.4.3 DmSNMP2B rescues DmSNMP1 loss-of-function in D. melanogaster 81

5 Discussion $\quad 84$

5.1 The organization of an olfactory sensillum of T. castaneum 84

5.2 Identification of six SNMPs in T. castaneum 86

5.3 TcSNMP1z is dissimilar to TcSNMP1 and TcSNMP2 homologs 87 
5.4 TcSNMP expressing cells are probably housed in sensilla trichodea and sensilla basiconica 88

5.5 TcSNMP2 expressing cells weren't detectable in antennal club segments 89

5.6 The potential role of TcSNMPs in supporting cells 90

5.7 TcSNMP1d expression is partially correlated with TcSNMP1z expression 91

5.8 The functional role of neuronal T. castaneum SNMPs in odor detection 92

5.8.1 The functional role of neuronal T. castaneum SNMPs in pheromone detection 93

5.8.2 T. castaneum SNMPs are involved in non pheromone odor detection 95

5.8.3 TcSNMP1a might be more important for female beetles 95

5.8.4 Outlook 96

5.9 T. castaneum SNMP1 diversification might mirror specialized coleopteran ecologic adaptation 96

5.10 TcSNMPs have conserved subdomains in the extracellular loop 98

5.11 DmSNMP2 is able to compensate for loss of DmSNMP1 function in cVA detection in $\begin{array}{ll}\text { the antenna } & 99\end{array}$

5.12 T. castaneum SNMP2 influences the odor response profile of the D. melanogaster pheromone receptor OR67d in snmp1 mutant flies 102

5.13 T. castaneum SNMP1a and SNMP1b.1 influence presynaptic inhibition within the antennal lobe of snmp1 mutant D. melanogaster 103

$\begin{array}{ll}5.14 \text { Novel roles of SNMPs } & 105\end{array}$

6 Supplemental 106

6.1 Full-length cDNA sequences and amino acid sequences of TcSNMPs 106

$\begin{array}{ll}\text { 6.1.1 TcSNMP1a } & 106\end{array}$

$\begin{array}{ll}\text { 6.1.2 TcSNMP1b.1 } & 107\end{array}$

$\begin{array}{ll}\text { 6.1.2 TcSNMP1b.2 } & 108\end{array}$

$\begin{array}{ll}\text { 6.1.2 TcSNMP1c } & 109\end{array}$

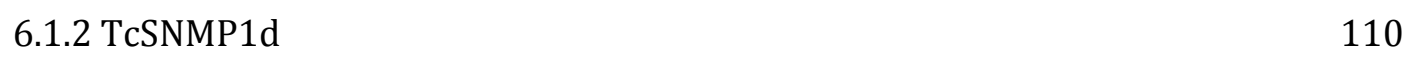

$\begin{array}{ll}\text { 6.1.2 TcSNMP1z } & 111\end{array}$

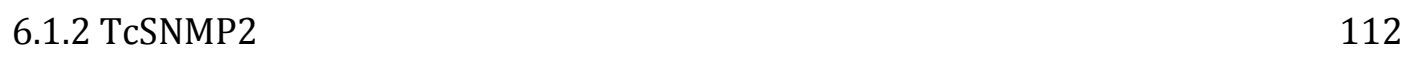

6.2 Comparison of the full-length TCSNMP cDNA sequences with the respective in silico annotation 113

\begin{tabular}{ll}
7 Bibliography & 119 \\
\hline
\end{tabular}

8 Acknowledgement 137

9 Curriculum vitae 139 


\section{List of Figures}

Figure 2.1 The antenna of T. castaneum 5

$\begin{array}{lll}\text { Figure 2.2 } & \text { Model of an olfactory sensillum } & 7\end{array}$

Figure 2.3 Possible signal transduction mechanisms of insect OSNs 10

$\begin{array}{lll}\text { Figure 2.4 The antennal lobe, the primary olfactory center in the insect brain } & 12\end{array}$

Figure 2.5 Current model of SNMP1 function in the pheromone detection 15 system

$\begin{array}{lll}\text { Figure 2.6 } & \text { Transcriptome data of } T \text {. castaneum SNMPs }\end{array}$

Figure 4.1 Full-length cDNA sequences of the isolated TcSNMPs 39

Figure 4.2 Alignment of amino acid sequences of all obtained TcSNMP 41 proteins

Figure 4.3 Amino acid identities among TcSNMP proteins 42

Figure 4.4 Phylogenetic analysis of SNMPs and other CD36 proteins 45

Figure 4.5 FISH visualizing marker transcripts of OSNs (Orco) and secretory 48 supporting cells $(O B P 9 b)$ in the most distal $T$. castaneum antennal segment

Figure 4.6 FISH and nuclear DAPI staining on T. castaneum antenna $\quad 49$

Figure 4.7 Double FISH visualizing marker transcripts of OSNs (OrCo) and $\quad 50$ secretory supporting cells $(O B P 9 b)$ in $T$. castaneum club segments

Figure 4.8 Expression pattern of SNMP1a 53

Figure 4.9 Expression pattern of SNMP1b 55

Figure 4.10 Expression pattern of SNMP1C 57

Figure 4.11 Expression pattern of SNMP1d 59

$\begin{array}{lll}\text { Figure 4.12 } & \text { Expression pattern of } S N M P 1 z & 61\end{array}$

Figure 4.13 Cellular organizations of different SNMPs within one segment 64

Figure 4.14 EAG responses of RNAi treated T. castaneum to Tribolium odors $\quad 67$

Figure 4.15 EAG responses of RNAi treated T. castaneum to fatty food odors $\quad 69$

Figure 4.16 EAG responses of RNAi treated T. castaneum to plant odors 70

Figure 4.17 Validation of the RNAi-induced knockdown 72

Figure 4.18 Functionality of the D. melanogaster rescue assay system 75

Figure 4.19 Rescue abilities of different SNMPs 77

Figure 4.20 Modified odor responses of the DA1 glomerulus to 1-hexanol 79

Figure 4.21 Modified odor responses of the DA1 glomerulus to isoamyl acetate 80 
induced by TcSNMPs

Figure 4.22 Structure prediction of different SNMPs of D. melanogaster 83

Figure 5.1 Model of an olfactory sensillum of T. castaneum 85

Figure 5.2 Potential chimeric constructs for further analysis 101

Figure S1 Comparison of the obtained TcSNMP1a cDNA sequence with the in 113 silico annotation

Figure S2 Comparison of the obtained TcSNMP1b.1 cDNA sequence with the in silico annotation

Figure S3 Comparison of the obtained TcSNMP1c cDNA sequence with the in silico annotation

Figure S4 Comparison of the obtained TcSNMP1d cDNA sequence with the in silico annotation

Figure S5 Comparison of the obtained TcSNMP1z cDNA sequence with the in silico annotation

Figure S6 Comparison of the obtained TCSNMP2 cDNA sequence with the in silico annotation

\section{List of Tables}

Table 1

Oligonucleotides used in this study

Table 2

D. melanogaster balancer lines used in this study

Table 3

D. melanogaster initial lines used in this study

Table 4

D. melanogaster parental lines of flies used for

functional analysis in this study 


\section{List of abbreviations}

\begin{tabular}{|c|c|}
\hline${ }^{\circ} \mathrm{C}$ & degree in Celsius \\
\hline AL & antennal lobe \\
\hline Amp & ampicillin \\
\hline $\mathrm{bp}$ & base pair \\
\hline CHAPS & 3-[(3-Cholamidopropyl)dimethylammonio]-1-propanesulfonate \\
\hline cVA & cis-vaccenyl acetate \\
\hline DAPI & 4',6-Diamidin-2-phenylindol \\
\hline DIG & digoxigenin \\
\hline Dm/Dmel & Drosophila melanogaster \\
\hline DMD & 4-8-dimethyl decanal \\
\hline DNA & desoxyribonucleic acid \\
\hline dNTP & desoxynucleosidtriphosphate \\
\hline ds & double stranded \\
\hline EAG & electroantennography \\
\hline E. coli & Escherichia coli \\
\hline e.g. & exempli gratia \\
\hline EDTA & ethylenediaminetetraacetic acid \\
\hline FISH & fluorescence in situ hybridization \\
\hline g & gram \\
\hline g & gravity \\
\hline GABA & gamma-aminobutyric acid; \\
\hline h & hour \\
\hline \multirow[t]{2}{*}{ HNPP/Fast Red } & 2-hydroxy-3-naphthoic acid-2'-phenylanilide phosphate/4-chloro- \\
\hline & 2-methylbenzene diazonium hemi-zinc chloride salt \\
\hline HRP & horseradish peroxidase \\
\hline $\mathrm{kb}$ & kilo base \\
\hline l & liter \\
\hline LB & lysogeny broth \\
\hline LN & local inter-neurons \\
\hline LSM & laser scanning microscope \\
\hline M & molar \\
\hline $\mathrm{m}$ & meter \\
\hline $\mathrm{m}$ & milli \\
\hline
\end{tabular}




\begin{tabular}{|c|c|}
\hline $\min$ & minute \\
\hline $\mathrm{nm}$ & nanometer \\
\hline OBP & odorant binding protein \\
\hline OR & odorant receptor \\
\hline Orco & odorant receptor co-receptor \\
\hline ORF & open reading frame \\
\hline OSN & olfactory sensory neurons; PBS, phosphate buffered saline \\
\hline PBP & pheromone binding protein \\
\hline PCR & polymerase chain reaction \\
\hline $\mathrm{pH}$ & negative logarithm of proton concentration \\
\hline PN & projection neuron \\
\hline RACE & rapid amplification of cDNA ends \\
\hline RNA & ribonucleic acid \\
\hline RPKM & reads per kilo base per million; \\
\hline RT & room temperature \\
\hline s & second \\
\hline SDS & sodium dodecyl sulfate \\
\hline SNMP & sensory neuron membrane protein \\
\hline SSC & saline-sodium citrate \\
\hline SSR & single sensillum recordings \\
\hline TBS & TRIS buffered saline \\
\hline Tc/Tcas & Tribolium castaneum \\
\hline TMD & transmembrane domain \\
\hline TRIS & tris(hydroxymethyl)aminomethane \\
\hline TSA & Tyramide Signal Amplification \\
\hline UAS & upstream activation sequence \\
\hline UTR & untranslated region \\
\hline $\mathrm{V}$ & Volt \\
\hline$\mu$ & micro \\
\hline
\end{tabular}




\section{Summary}

Olfaction, the detection of odors is pivotal for insects, since it leads them to food sources or mating partners and triggers important behaviors. For the detection of pheromones, CD36-related sensory neuron membrane proteins (SNMPs) play a crucial role. Despite their necessity, the functional role of SNMPs is not yet fully understood. Generally, two single SNMPs, namely SNMP1 and SNMP2, have been described in insects, whereas the pest beetle Tribolium castaneum has six genes expressed that encode putative SNMPs as indicated by transcriptome analysis.

To investigate these proteins in more detail, I determined the actual full-length transcript sequences of the six T. castaneum SNMPs by RACE-PCR on antennal cDNA pools. This enabled the identification of the actual TcSNMP protein sequences and allowed the phylogenetic classification of these proteins. To analyze the expression patterns of the T. castaneum SNMPs, I established a reliable double fluorescence in situ hybridization protocol for adult antennae of T. castaneum, which I used to visualize and characterize the TcSNMP-expressing cells. This revealed that - besides TCSNMP2 for which no specific expression pattern could be determined - the TCSNMPS are expressed differently with regard to number of cells, antennal segments, as well as cell types, which suggests a distinct role of the diversified TcSNMPs. In addition, by loss-of-function experiments it became apparent that the neuronal TcSNMPs are involved in the detection of fatty odors, emitted from various ecological important sources, such as pheromones, social odors, as well as potential food odors. These findings extend the known involvement of SNMPs for pheromone detection to other odors apart from pheromones.

Moreover, functional analyses of heterologously expressed TcSNMPs in snmp1 mutant D. melanogaster showed that the beetle TcSNMPs were not able to complement lacking DmSNMP1 function. This indicated that the diversified beetle TcSNMPs evolved differentially to the fly DmSNMP1, to probably serve different functions. Remarkably, instead of rescuing the snmp1 mutant situation, some TcSNMPs modified the odor response profile of the pheromone detecting neurons 


\section{Summary}

of D. melanogaster towards non-pheromone odors. This implicates that SNMPs have functions in addition to the known mediation of pheromones.

Heterologous expression of DmSNMP2 isoform B and C in snmp1 mutant $D$. melanogaster with subsequent functional analyses revealed that the DmSNMP2 isoform $\mathrm{B}$, but not isoform $\mathrm{C}$, is able to rescue the snmp1 mutant situation. Via protein prediction tools I calculated the tertiary structure of these isoforms, which pointed to a particular alpha helix forming the functional region. The DmSNMP1 protein contains a corresponding alpha helix which offers the great opportunity for elucidating this putative functional area of DmSNMP1 in further analyses. 


\section{Introduction}

Olfaction is the ability to perceive volatile compounds emitted from the environment. This chemical sense occurs in its most simple form in bacteria that detect for example airborne ammonia that is needed for their growth (Nijland and Burgess, 2010). The more developed sense of smell of complex organisms allows the detection of various odors that helps to find mating partners, to avoid environmental threats or toxins, and to localize potential food sources (Kurtovic et al., 2007; Stensmyr et al., 2012; Zarzo, 2007). In this context, the olfactory systems of insects have an outstanding importance regarding human welfare, since they for example mediate attraction of insects to humans and thereby allow the insects to transmit deadly diseases, such as malaria (Carey et al., 2010), or they lead pest insects to their desired food or egg laying substrate, potentially causing agricultural damage (Atwal, 1976; Zettler and Cuperus, 1990).

But not only because of this, has the olfactory system of insects become an important model in neuroscience. Compared to vertebrates, olfactory systems of insects are easily accessible and organized in a less complex manner (Martin et al., 2011b). Furthermore, olfaction in insects is often tightly coupled to stereotypical behavioral changes (Hall, 1994; Stensmyr et al., 2012). Therefore insect model systems allow studying the sense of smell at different levels of odor perception: from analyses about how an odor is detected by a single neuron, over processing of olfactory neuronal signals in the brain, to the final behavioral change (Heimbeck et al., 2001; Martin et al., 2011b; Ronderos et al., 2014). Especially the pioneering studies on the extraordinarily sensitive sex-pheromone detecting system of lepidopterans revealed basic principles about the functionality of the insect olfactory system (Hildebrand, 1996; Steinbrecht, 1996; Steinbrecht and Gnatzy, 1984; Steinbrecht et al., 1992). Meanwhile, powerful neurogenetic tools are available in insects, allowing the study of the neuronal activity of single neurons or complex neuronal circuits in odor processing (Akerboom et al., 2012; Lebreton et al., 2014; Pregitzer et al., 2012). Here, the neurogenetic model organism Drosophila melanogaster has to be mentioned. This fly is amenable to various genetic 
Introduction

manipulations, such as gene silencing, misexpression systems, thermogenetic or optogenetic activation or inhibition of single neurons or neuron classes and thereby allowing easy monitoring of neuronal activity (Pauls et al., 2015; Reichert, 2014).

In the present study, the olfactory system of the red flour beetle Tribolium castaneum, a pest species infesting stored agricultural products, was investigated (Zettler and Cuperus, 1990). Over the past years, T. castaneum - a representative of the largest insect order Coleoptera - together with its fully annotated genome (Kim et al., 2010; Richards et al., 2008; Wang et al., 2007) has developed to a beetle model organism used by developmental biologists (Denell, 2008; Dippel et al., 2014). Powerful genetic tools such as systemic RNA interference (Bucher et al., 2002; Tomoyasu and Denell, 2004) and misexpression systems (Schinko et al., 2010, 2012) are available in T. castaneum. This will facilitate the detailed examination of the olfactory system of this pest beetle, with regard to differences or similarities to the mechanisms of odor detection described in Diptera and Lepidoptera.

\subsection{The peripheral olfactory system of insects}

Olfaction in insects is mediated by olfactory sensory neurons (OSNs) located in the olfactory head appendages, typically the antennae and mouthparts (figure 2.1; Carey and Carlson, 2011). The dendrites of the OSNs are housed in hair like structures, the so called "olfactory sensilla" (figure 2.2; Steinbrecht, 1996). Olfactory sensilla allow the entry of odorants through characteristic pores (Chapman et al., 2013) and occur in different shapes. Based on their shape, they are classified into different morphological subtypes, such as long hair shaped sensilla trichodea, peg formed sensilla basiconica, and peg-like sensilla coeloconica, which protrude from deep pits (Steinbrecht, 1996; Venkatesh and Naresh Singh, 1984).

Whether the specific shape of a given sensillum type contributes to the detection of special classes of odorants has not been clarified. However, trichoid sensilla of various lepidopteran species as well as those of the vinegar fly D. melanogaster 
have been described to house dendrites of OSNs that are linked to the detection of pheromones (Clyne et al., 1997; Ha and Smith, 2006; Steinbrecht and Gnatzy, 1984). In contrast, D. melanogaster OSNs that are housed in s. basiconica are mainly known for their detection of odors emitted from food sources (de Bruyne et al., 2001).

On the antenna of T. castaneum, which consists of eleven segments (figure 2.1; Angelini et al., 2009), olfactory sensilla are covering the surface of the most distal three segments (segments 9, 10, and 11), called the club segments. Here, s. trichodea, s. basiconica and s. coeloconica have been identified (Dippel et al. in preparation). Whether OSNs housed in these sensilla show a similar response profile as in D. melanogaster and lepidopteran species has not been investigated, yet.

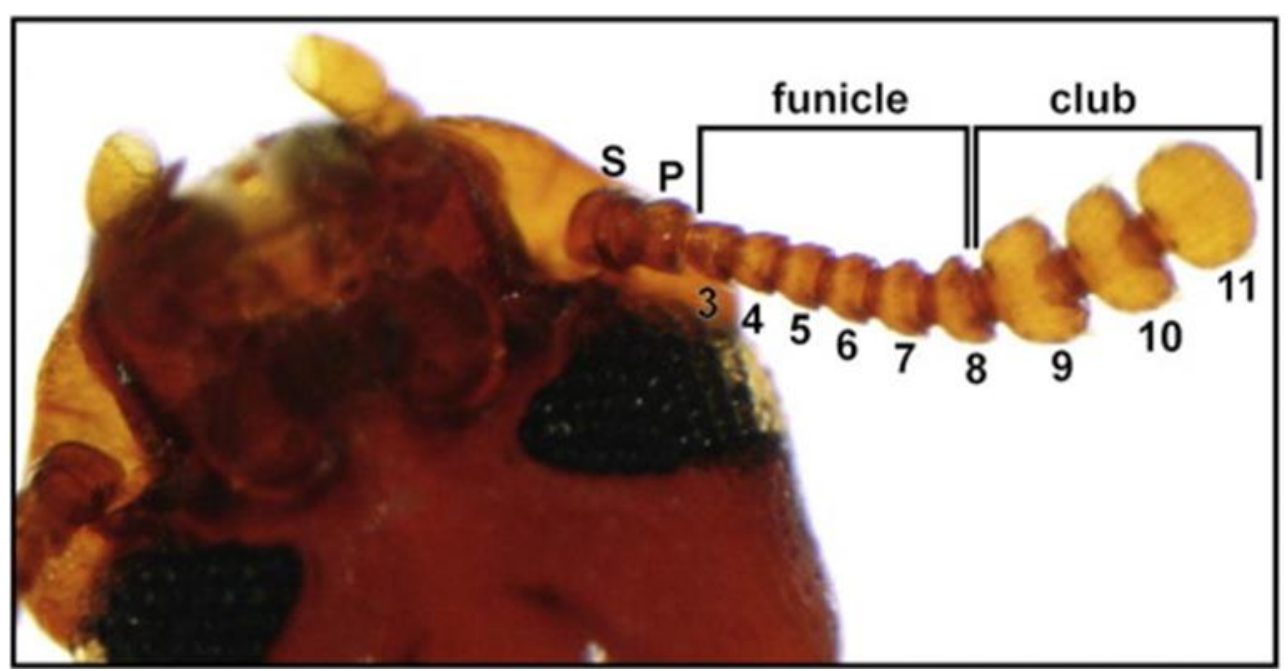

Figure 2.1 The antenna of $T$. castaneum

Shown is the adult head and all eleven segments of the antenna of T. castaneum. Segment one is also named scape (S), segment two pedicel (P), segments 3-8 together funicle and segments 9-11 together club. The club segments are covered with olfactory sensilla (Dippel et al. in preparation). Adapted from (Angelini et al., 2009).

\subsubsection{Odorant binding proteins (OBPs) as carriers for odors in the sensillar lymph}

Beneath the olfactory sensilla the cell bodies of the OSNs and three kinds of supporting cells, the thecogen cell, the trichogen cell, and the tormogen cell are located (figure 2.2; Merritt, 1989; Steinbrecht, 1996). While the thecogen cell 
Introduction

builds a sheath around the cell body and dendrites of the OSNs, the other two kinds of supporting cells are believed to produce and secrete the sensillum lymph that fills the olfactory sensillum (Steinbrecht, 1996; Steinbrecht and Gnatzy, 1984). The sensillar lymph imbeds the dendrites of the OSNs and builds a hydrophilic barrier for most odorants that enter the hair through pores (Steinbrecht, 1996). Therefore it is believed that odorant binding proteins (OBPs) act as carriers for hydrophobic odorants that bind and transfer them to the dendrite of the OSNs (Kaissling, 2001; Pelosi et al., 2006; Vogt, 2003). OBPs are small, soluble proteins that are produced by secretory supporting cells and occur in high concentration in the sensillar lymph (figure 2.2; Galindo and Smith, 2001; Kim and Smith, 2001; Vogt et al., 2002). Because the sensillum lymph of every olfactory sensillum is isolated, OSNs housed in different sensilla can be surrounded by different OBPs (Sakurai et al., 2014). The mechanism of odorant binding and release has been characterized in detail for the pheromone binding protein 1 (PBP1) of Bombyx mori. It is hypothesized that PBP1 changes its conformation depending on the $\mathrm{pH}$, facilitating the binding of the pheromone at the cuticle (neutral $\mathrm{pH}$ ) and the release of pheromone at the dendritic membrane of the OSN (acidic pH) (Tegoni et al., 2004; Wojtasek and Leal, 1999). The first OBP was identified in the sensillar lymph of the polyphemus moth Antheraea polyphemus (Vogt and Riddiford, 1981). Meanwhile, OBPs have been identified in more than 40 other insect species belonging to eight different orders (Galindo and Smith, 2001; Hekmat-Scafe et al., 2002; Leitch et al., 2015; Liu et al., 2015; McKenzie et al., 2014; Pelosi and Maida, 1995; Pelosi et al., 2006; Vieira and Rozas, 2011). In T. castaneum the expression of 49 different OBPs has been confirmed by transcriptome analysis (Dippel et al., 2014). 


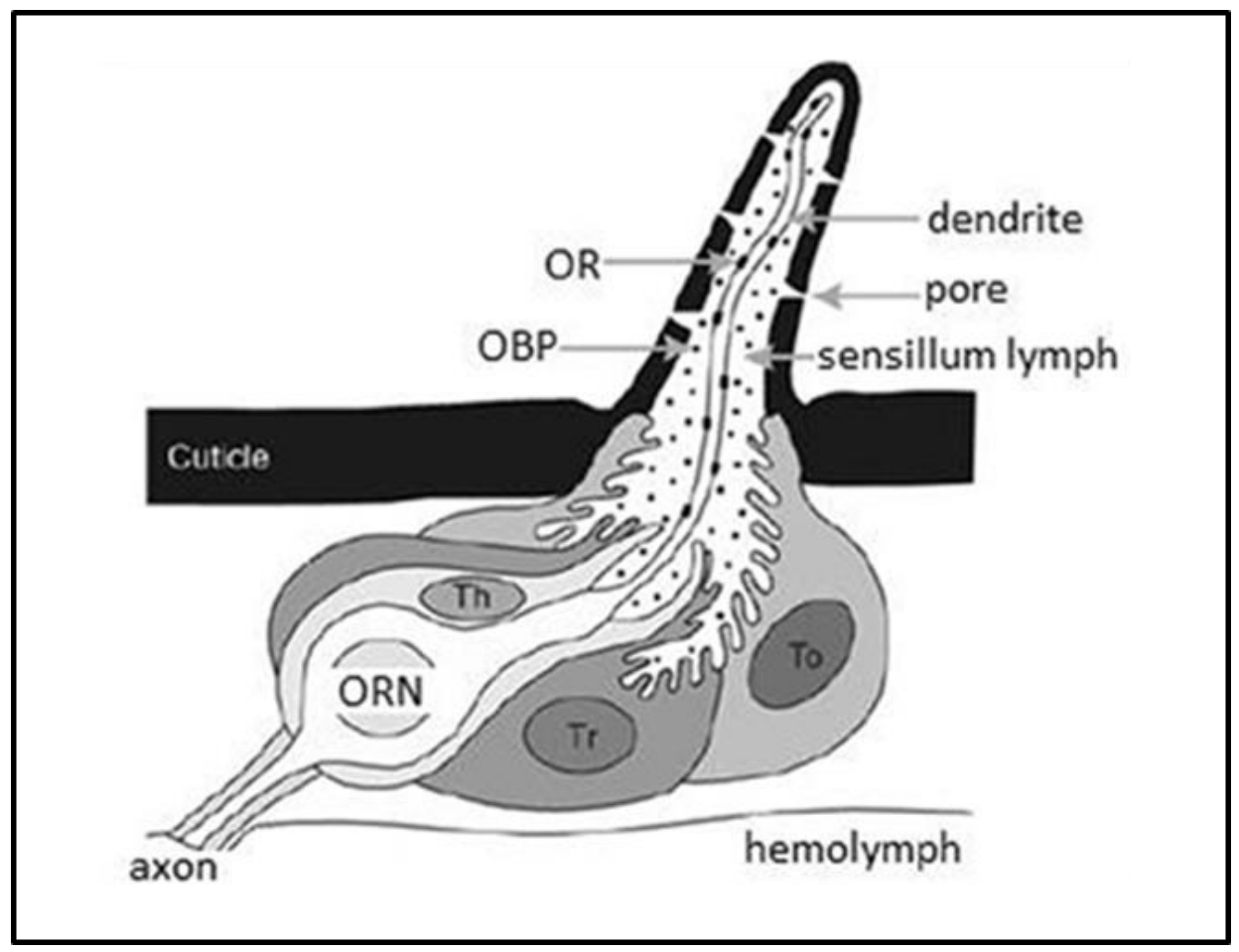

Figure 2.2 Model of an olfactory sensillum

Shown is a model of a moth olfactory sensillum. Depicted is the cellular organization of the different supporting cells that surround the cell body of the OSN (in the model "ORN"): the tormogen (To), trichogen ( $\mathrm{Tr}$ ), and thecogen cells (Th). While the thecogen cell ensheaths the OSN, tormogen and trichogen cells produce and secrete the sensillum lymph including the OBPs. The dendrites of the OSN, containing the odorant receptors (OR), are imbedded in the sensillum lymph. Adapted from (Sakurai et al., 2014).

\subsubsection{Odor detection at the OSN by odorant receptors}

At the OSN the recognition of odorants is carried out by odorant receptors (ORs) embedded in the dendritic membrane (figure 2.2). The insect ORs were first characterized in D. melanogaster. They belong to a class of seven-trans-membranedomain-receptors (7TMD) (Clyne et al., 1999; Gao and Chess, 1999; Vosshall, 2000; Vosshall et al., 1999). Meanwhile ORs were identified in plenty of other insects, e.g. different lepidopteran species (Grosse-Wilde et al., 2011; Gu et al., 2014; Zhang et al., 2014a), dipteran species (Andersson et al., 2014; Carey et al., 2010; Leitch et al., 2015), hymenopteran species (Smith et al., 2011, 2011; Zhang et al., 2014b), and coleopteran species (Andersson et al., 2013; Gu et al., 2015; Liu et al., 2015). Transcriptome analysis of $T$. castaneum revealed the expression of 129 ORs in the antenna (Dippel et. al. in preparation). 
Introduction

Subsequent functional analyses of ORs from insects belonging to the order Lepidoptera and Diptera were performed either in their native context (de Bruyne et al., 2001; Silbering et al., 2011; Yao et al., 2005) or in heterologous expression systems (Hallem and Carlson, 2006; Hallem et al., 2004; Pregitzer et al., 2014; Ronderos et al., 2014). These analyses revealed that ORs have ligand profiles of varying tuning width, which means that some ORs are broadly tuned and respond to a large number of different odors while others are narrowly tuned, responding to just a small set of odors (Carey et al., 2010; Hallem and Carlson, 2006; Wang et al., 2010). Some of the most selective ORs detect social odors. One very well characterized narrowly tuned OR is the D. melanogaster OR67d (DmOR67d). Dm0R67d is described to be narrowly tuned to the aggregation pheromone cisvaccenyl acetate (cVA) that is produced by male flies and regulates mating behavior of both sexes (Clyne et al., 1997; van der Goes van Naters and Carlson, 2007; Ha and Smith, 2006; Kurtovic et al., 2007). Remarkably, DmOR67d expressing neurons are not just highly specialized to cVA, but are also inhibited by most other odors (Vosshall et al., 1999). None of the identified T. castaneum ORs has been functionally characterized, yet.

\subsection{Signal transduction in OSNs}

After binding of a given odorant, the OR has the function to signal the identity and quantity of the odorant by inducing electrical activity in OSNs. In vertebrate OSNs, odorant binding at the OR activates the receptor-coupled G-protein and adenylyl cyclase (AC) (Pace et al., 1985; Sklar et al., 1986). Thereby cyclic adenosine monophosphate (cAMP) is produced, which opens ion channels that cause a depolarizing influx of sodium and calcium ions. Finally, the calcium ion influx opens calcium induced chloride channels that facilitate a chloride efflux and thereby a stronger depolarization (Kleene and Gesteland, 1991; Pifferi et al., 2010). Due to the fact that insect ORs, like vertebrate ORs, possess seven TMDs that are typical for G-Protein coupled receptors (GPCRs), it was believed that insect ORs trigger a similar signal transduction cascade (Nakagawa and Vosshall, 2009). However, several studies about the OR protein topology showed that the insect 
ORs have an inverted topology compared to other GPCRs (Benton, 2006; Lundin et al., 2007; Smart et al., 2008). While GPCRs, including vertebrate ORs, have a cytoplasmic $\mathrm{C}$-terminus and an extracellular $\mathrm{N}$-terminus, the termini of the insect ORs are located the other way around. This raised the suggestion that the insect ORs might use different signal transduction mechanisms than GPCRs (Nakagawa and Vosshall, 2009). Furthermore, in contrast to vertebrate OSNs, it was revealed that every insect OSN that expresses a ligand binding OR also expresses the highly conserved odorant receptor coreceptor (Orco) that does not bind to odors (Vosshall et al., 1999).

Orco is necessary for trafficking ORs to the dendritic membrane of the OSN and builds a heteromer with the ligand binding OR in a yet unknown stoichiometry (Benton et al., 2006; Mukunda et al., 2014; Neuhaus et al., 2005). Continuative functional analyses revealed that Orco forms an ion channel that can be gated by an activated OR (Jones et al., 2011; Sato et al., 2008; Wicher et al., 2008). This led to the following model of signal transduction in insect OSNs: binding of an odorant to a specific OR leads to a conformational change within the OR/Orco complex that, in turn, opens the ion channel (figure $2.3 \mathrm{~A}$ ). The resulting influx of sodium and calcium ions then leads to the depolarization of the OSN (Kaupp, 2010). However, an influence of G-protein-coupled signal transduction cascades in insect OSNs has not been ruled out. A different model assumes that Orco alone builds an ion channel that is directly gated in the presence of high odor concentrations. In contrast, low odor concentrations lead to G-protein activation by the ligand binding $\mathrm{OR}$, resulting in a signal amplification by the production of second messengers that are able to open the ion channel (figure $2.3 \mathrm{~B}$; Getahun et al., 2013; Ignatious Raja et al., 2014; Martin and Alcorta, 2011; Stengl, 2010; Wicher et al., 2008, 2009).

Recent publications even extend the role of Orco to a modulating protein, by suggesting that second messenger systems can lead to a phosphorylation of Orco which may enhance the responses towards a given odor (Getahun et al., 2013; Martin and Alcorta, 2011; Wicher et al., 2009). 


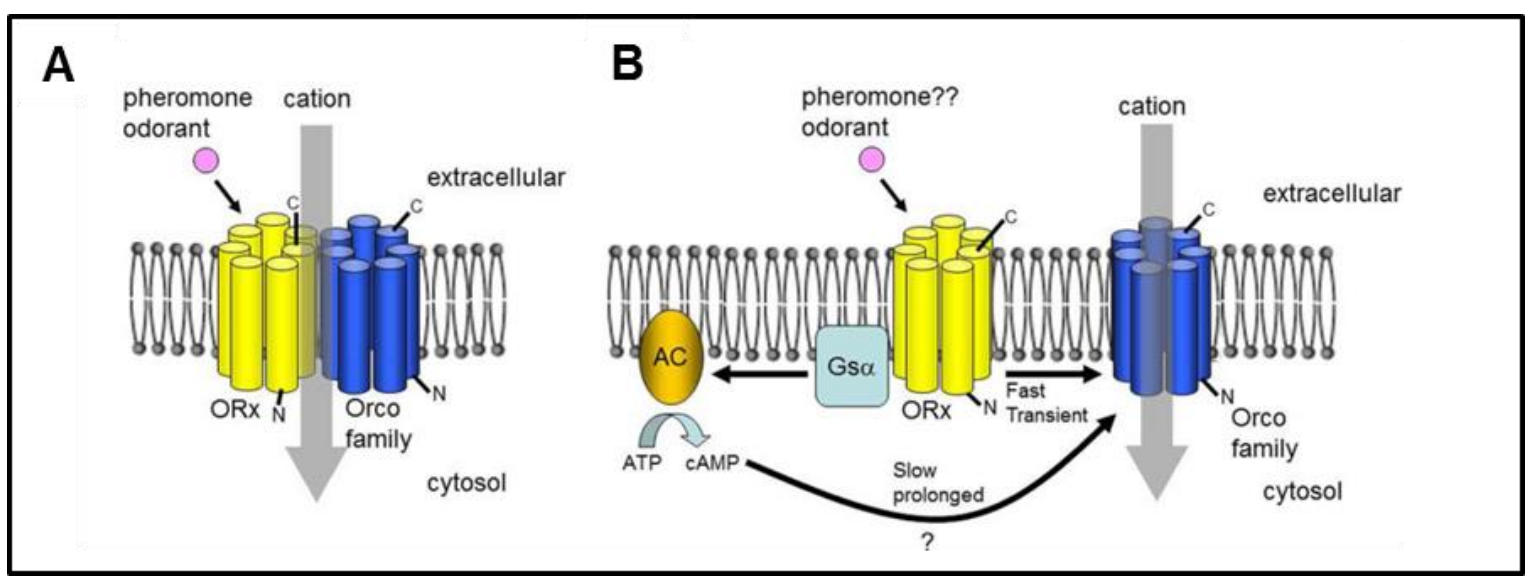

Figure 2.3 Possible signal transduction mechanisms of insect OSNs

Different models exist concerning the odor induced signal transduction in insect OSNs.

A Shown is one suggested signal transduction mechanism in which the OR/Orco heteromer forms an odorant-gated cation channel. Here, binding of a pheromone or an odorant to the OR results in the opening of the channel, allowing the influx of cations into the cytosol, leading to OSN depolarization.

B Depicted is another hypothesized signal transduction mechanism in which the cation channel is formed exclusively by Orco. Here, upon odor binding, the OR opens the cation channel for fast responses in the presence of high odorant concentrations. In case of low odor concentrations, a second messenger cascade is started by the activated OR for signal amplification.

Adapted from (Sakurai et al., 2014).

\subsection{Odor processing in the antennal lobe}

The primary olfactory center in the insect brain, the antennal lobe (AL), is composed of glomeruli (Tanaka et al., 2012). Glomeruli are spherical structures of high synaptic density, where the electrical responses of OSNs become integrated and modified before they are transmitted to higher brain areas (Schachtner et al., 2005). Axons of all OSNs that express the same OR converge into the same specific glomerulus (figure 2.4; Gao et al., 2000). Size, shape and localization of a given glomerulus are species specific, a fact that allows individual glomerulus identification (Berg et al., 2002; Laissue et al., 1999).

Imaging studies in different insect species revealed that a given odorant generates complex and identical patterns of activated glomeruli (Galizia et al., 2000; Sachse et al., 1999). Importantly, some odorants stimulate many classes of OSNs and therefore many glomeruli, while other odors cause more specific and restricted activation patterns. For example cVA is detected by only a small number of OSNs, such as the OR67d expressing OSNs, and consequently just activates a small group 
of glomeruli, in the given example the glomerulus DA1 (Datta et al., 2008; Kurtovic et al., 2007).

Within glomeruli, OSNs synapse to local interneurons (LNs) and projection neurons (PNs) (Aungst and Spehr, 2005). LNs are located lateral to the AL. They have no projections outside the AL but build a $\mathrm{LN}$ network that interconnects different glomeruli (Anton and Homberg, 1999; Meyer et al., 2013). Most LNs build inhibitory connections between glomeruli, allowing that an excited glomerulus inhibits other glomeruli via inhibitory LNs (Sachse and Galizia, 2002). This serves the contrast enhancement between glomeruli. LNs belong to different classes regarding their connectivity, physiological properties, and neurotransmitter profiles (Chou et al., 2010; Seki et al., 2010). The majority of LNs are described to release gamma-aminobutyric acid (GABA) (Mikael A Carlsson, 2010). Remarkably, pheromone sensing OSNs of D. melanogaster have been shown to express high levels of GABA receptors, which correlates with a high level of presynaptic inhibition in these OSNs (Wilson and Laurent, 2005). Aside from the GABAergic LNs, also glutamatergic or histaminergic inhibitory LNs have been described (Liu and Wilson, 2013; Root et al., 2008; Sachse et al., 2006). Furthermore neuropeptides are believed to modify the effect of these transmitters at the synapse (Binzer et al., 2014; Ignell et al., 2009; Joachim Schachtner, 2005; Mikael A Carlsson, 2010; Wilson, 2013). The processed electrical OSN responses are transmitted to PNs that have dendritic connections in the AL and axonal connections to higher brain areas, such as the calyces of the mushroom body, where olfactory memory is formed, and the lateral horn where behaviorally relevant olfactory information is processed (Guven-Ozkan and Davis, 2014; Strutz et al., 2014). 


\section{Introduction}

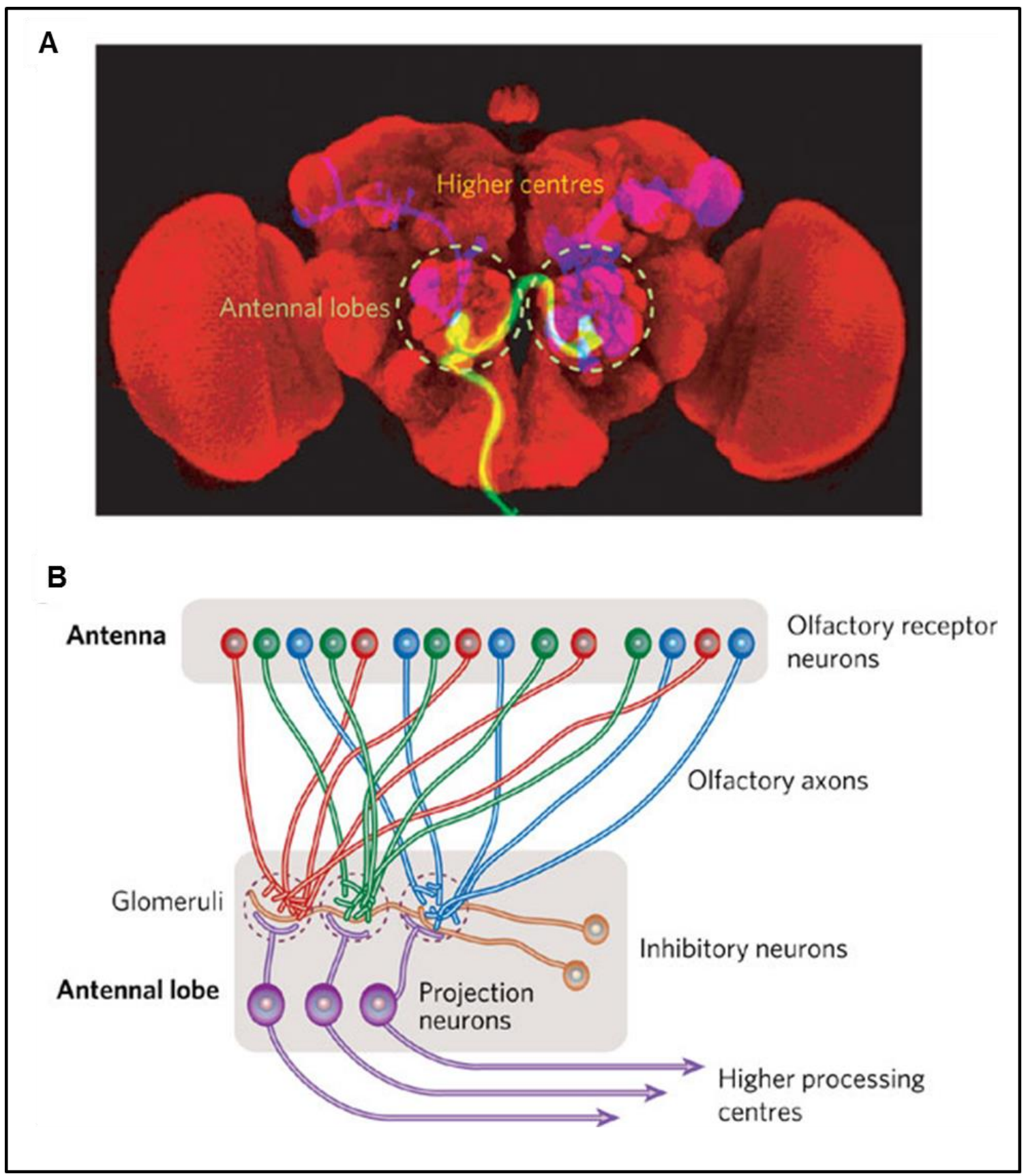

Figure 2.4 The antennal lobe, the primary olfactory center in the insect brain

A Antibody stainings targeting different structures of the D. melanogaster brain. Axons of OSNs expressing the same OR project to the same glomerulus within the antennal lobe (framed by a light green dotted circle). An unknown subclass of OSNs is labelled in yellow. In the antennal lobe the OSNs synapse to projection neurons (purple) and interneurons (not colorized). The projection neurons project to higher brain centers.

B Model of the glomerular organization within the antennal lobe. All OSNs with the same OR (indicated by red, green or blue colorization) converge to the same glomerulus. In the glomerulus, the electrical responses of OSNs become processed by local interneurons that connect different glomeruli (LNs, orange; in the image referred to as 'Inhibitory neurons'). Finally, the processed odor response is projected to higher brain centers by projection neurons (purple).

Adapted from (Bargmann, 2006). 


\subsection{Sensory neuron membrane proteins (SNMPs)}

Strong innate behavioral responses are mediated by OSNs that express specific ORs, that are narrowly tuned to pheromones, non-pheromonal social odors or important environmental odors (Hansson and Stensmyr, 2011; Kurtovic et al., 2007; Ronderos et al., 2014; Stensmyr et al., 2012). Remarkably, in D. melanogaster several of the identified OSNs that have these characteristics express a CD36 related receptor, namely the sensory neuron membrane protein 1 (SNMP1), in addition to the OR/Orco complex (Benton et al., 2007; Jin et al., 2008; Kurtovic et al., 2007; Ronderos et al., 2014). More precisely, SNMP1 expressing neurons have been primarily found in trichoid sensilla that are connected to the detection of pheromones, fly extracts (unknown fly body odors), and farnesol (Benton et al., 2007; van der Goes van Naters and Carlson, 2007; Ronderos et al., 2014). Farnesol is an odor emitted by ripe citrus peels, a potential egg laying site for $D$. melanogaster (Ronderos et al., 2014). SNMP1 expression in pheromone reactive neurons has also been described in different moth species, including the polyphemus moth Antheraea polyphemus, where the first SNMP1 had been identified (Forstner et al., 2008; Rogers et al., 1997, 2001).

A second SNMP type was identified in the antenna of the tobacco hornworm Manduca sexta, which shares 25 - $30 \%$ of amino acids with the cognate SNMP1 protein and thus was named SNMP2 (Robertson et al., 1999; Rogers et al., 2001). Interestingly, SNMP2 proteins of moth species are not expressed in pheromone sensitive neurons but in secretory supporting cells that surround these neurons (Forstner et al., 2008).

Sequence analyses of SNMP proteins revealed that they possess two transmembrane domains, one big extracellular loop, and two short intracellular termini. Because of this protein structure and further sequence similarities, SNMPs have been grouped into the large CD36 protein family (Rogers et al., 2001). In insects as well as vertebrates, members of this family are mainly known for their capacity to recognize lipids, fatty acids or lipid-protein-complexes (Martin et al., 2011a). Members of the CD36 protein family are located in various physiological systems, such as the immune system (Stewart et al., 2010; Stuart et al., 2005), the gastrointestinal system (Guijarro et al., 2010; Nassir et al., 2007; Schneider et al., 
Introduction

2014), or various sensory systems (Fushiki, 2014; Sun et al., 2006). In vertebrates, CD36 has been shown to be involved in dietary lipid detection (Dramane et al., 2014), whereas in macrophages the CD36 protein detects specific lipids and lipoprotein components of bacterial cell walls (Hoebe et al., 2005). The fatty acid translocase (FAT), a further member of the CD36 protein family, plays a role in the uptake of long chain fatty acids by intestinal enterocytes (Chen et al., 2001). Insect specific CD36 proteins such as Croquemort and Debris buster are involved in phagocytosis of neuronal debris or apoptotic cells as well as phagosome maturation (Franc et al., 1999; Han et al., 2014). Another example is NinaD (Neither inactivation nor afterpotential-D), which mediates cellular uptake of carotenoids (Kiefer et al., 2002).

SNMP1 and SNMP2 homologs have been found in several holometabolous insect species (Vogt et al., 2009). Apart from members of different dipteran species (e.g. Drosophila melanogaster, Anopheles gambiae, Mayetiola destructor), lepidopteran species (e.g. Heliothis virescens, Bombyx mori), and hymenopterans (Apis mellifera), SNMP homologs were also identified in coleopteran species (Andersson et al., 2013, 2014; Benton et al., 2007; Forstner et al., 2008; Liu et al., 2015; Rogers et al., 2001; Vogt et al., 2009).

\subsubsection{SNMP function}

Based on the location of SNMP1 in the dendritic membrane of OSNs that are housed in trichoid sensilla and due to their resemblance to other members of the CD36 protein family, it has long been assumed that SNMPs could work as coreceptors that interact with fatty acid-derived pheromones or PBP/pheromone complexes and that they could mediate the recognition of the pheromone by the OR (Rogers et al. 1997). Functional analyses supported this assumption by demonstrating that pheromone-reactive neurons of D. melanogaster were not able to detect the aggregation pheromone cis-vaccenyl acetate (cVA) without a functional DmSNMP1 (Benton et al., 2007; Jin et al., 2008; Li et al., 2014). Remarkably, heterologous expression of pheromone receptors of the moth species Bombyx mori and Heliothis virescens in OSNs of D. melanogaster showed the same 
necessity of DmSNMP1 for the detection of the cognate pheromone (Benton et al., 2007; Li et al., 2014). In more detail, without a functional DmSNMP1, the moth pheromone receptors were not able to fulfill their function in $D$. melanogaster OSNs. All tested pheromones - bombykol from B. mori, (Z)-11-hexadecenal from $H$. virescens, and cVA from D. melanogaster - are typical pheromones comprised of fatty-acid derived hydrocarbon chains (Howard and Blomquist, 2005). Therefore, it has been suggested that this hydrocarbon chain is the common structure, which needs the specific and conserved SNMP1 function to be detected (Benton et al., 2007). Furthermore, it has been proposed in a recent study that DmSNMP1 is not just mediating the detection of pheromones, but also mediates their dissociation from the receptor (figure 2.5; Li et al., 2014).

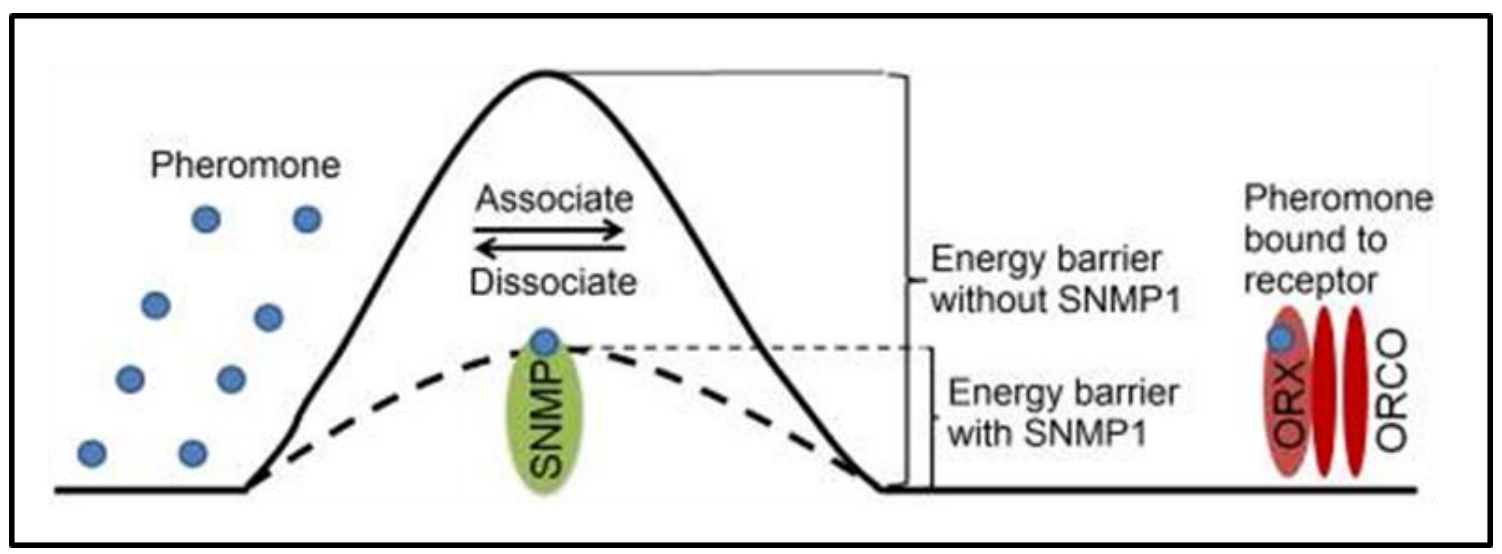

Figure 2.5 Current model of SNMP1 function in the pheromone detection system

SNMP1 proteins in pheromone sensitive neurons mediate pheromone detection (association) as well as dissociation of pheromones from the pheromone receptor (ORX). Adapted from (Li et al., 2014).

In addition to pheromone detection, SNMP1 function has been connected to the detection of non-pheromonal substances. Recently, it has been shown that SNMPs are also needed for normal response kinetics in farnesol perception. Farnesol is an odor present in the peel of citrus fruits, a potential egg laying substrate for $D$. melanogaster (Ronderos et al., 2014). However, OSNs lacking a functional DmSNMP1 were still activated by farnesol and did therefore not show the severe phenotype as snmp1 mutant OSNs of the pheromone detection system. 
Introduction

As mentioned above, SNMPs are not located exclusively in the OSNs but also in several secretory supporting cells that produce the sensillar lymph and control its composition (Benton et al., 2007; Forstner et al., 2008). Therefore, it was assumed that SNMPs in the membrane of supporting cells mediate the selective uptake of lipophilic molecules, such as degraded pheromones, and thereby may contribute to the cleaning of the sensillum lymph (Forstner et al., 2008). Interestingly, the SNMP types that are expressed in supporting cells differ between insect species. In the moth species $H$. virescens and $A$. polyphemus the SNMP1 homolog is expressed exclusively in OSNs, whereas SNMP2 is expressed in supporting cells (Forstner et al., 2008). In contrast in D. melanogaster, DmSNMP1 is expressed in both supporting cells and OSNs (Benton et al., 2007). Here, the D. melanogaster SNMP2 homolog is mainly expressed in the body and in a very low amount in the olfactory appendages (Chintapalli et al., 2007). DmSNMP2 has no described function in the olfactory system.

\subsubsection{SNMPs in T. castaneum}

During genome annotation of $T$. castaneum, seven genes were found that potentially encode for SNMPs (Nichols and Vogt, 2008; Vogt et al., 2009). Except for the hessian fly Mayetiola destructor, for which seven SNMPs were identified, for all other insects so far fewer SNMPs are described to be expressed (Andersson et al., 2013, 2014; Benton et al., 2007; Forstner et al., 2008; Liu et al., 2015; Rogers et al., 2001; Vogt et al., 2009). In general, a single SNMP1 and SNMP2 homolog each was found in various insect species (Benton et al., 2007; Forstner et al., 2008; Liu et al., 2015; Nichols and Vogt, 2008; Rogers et al., 2001; Vogt et al., 2009). Therefore the high number of putative SNMP genes in T. castaneum was an intriguing finding.

Recent tissue specific T. castaneum transcriptome data corrected the genome annotation, by showing that one of the annotated SNMPs (XP_969729) does not encode for an SNMP and that the gene model of TCSNMP1c (XM_001816389; Vogt et al., 2009) was a fusion of two separate TcSNMPs (Dippel et al. in preparation). This means that the expression of six TcSNMPs, namely TcSNMP1a, TcSNMP1b, 
TcSNMP1c, TcSNMP1d, TcSNMPX (XP_975606) and TcSNMP2 was confirmed by transcriptome analysis (figure 2.6; Dippel et al. in preparation). In the antennae, the main olfactory appendage of T. castaneum, these six TcSNMPs are expressed at varying levels. TcSNMP1a (2512.76 RPKM (reads per kilobase per million mapped reads)) and TcSNMP1c (791.42 RPKM) were the highest expressed TcSNMPs in antennal tissue, followed by a medium expression level of TcSNMP1b (23.53 RPKM) and TcSNMP1d (23.51 RPKM). Transcripts of TcSNMPX (2.3 RPKM) and TcSNMP2 (0.88 RPKM) were just expressed in low quantity in the antenna. Within the different tissue samples, all TcSNMP1 transcripts and the TcSNMPX transcript showed highest expression levels in the antennae and the mouthparts. Compared to these, TcSNMP2 is expressed much weaker in the antennae (0.88 RPKM) but strongly in the body (351.41 RPKM). Based on the chromosomal localization and its more TcSNMP1-like expression pattern (figure 2.6), the SNMPX (XP_975606) was preliminary named SNMP1z in the manuscript of Dippel et al. (in preparation). According to that, SNMPX will be named TcSNMP1z in this study.

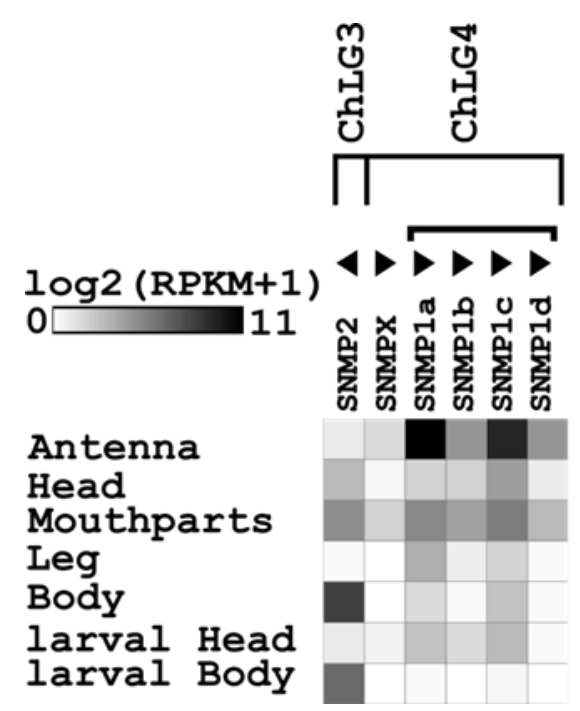

Figure 2.6 Transcriptome data of T. castaneum SNMPs

Shown are expression levels of the TCSNMP genes in different tissues and body parts: antenna, head with mouthparts, mouthparts, leg, body, larval head, and larval body. Expression levels are depicted as a heat map table in grey scale representing $\log 2[\mathrm{RPKM}+1]$ values. The darker the color of a table cell, the higher the amount of respective transcript in a given tissue. Arrowheads indicate the orientation of the ORF (open reading frame). As indicated by brackets, the SNMP2 gene locus is located on the 3rd chromosome, while SNMPX, SNMP1a, SNMP1b, SNMP1c, and SNMP1d are on the 4th. All SNMP1 gene loci (a-d) cluster closely together. Figure adapted fom Dippel et al. (in preparation). 
Introduction

\subsection{Aim of this thesis}

Despite the described importance of SNMPs in the olfactory systems of Diptera and Lepidoptera, the exact molecular mechanisms of SNMP function have not been elucidated yet. At the beginning of this work only a single SNMP1 homolog and SNMP2 homolog each had been described in all investigated insects. Therefore, the existence of six SNMPs in antennae of the pest beetle T. castaneum gives a great opportunity to investigate the olfactory function of these proteins in more detail, with special regards to differences and similarities to the described SNMPs of Diptera and Lepidoptera.

The expression of six TCSNMPs in antennal tissue was confirmed by transcriptome data, based on in silico annotations (Dippel et al. in preparation). As precondition for subsequent analyses, I wanted to determine the actual full-length transcript sequences of the six TCSNMPs by RACE-PCR on antennal cDNA pools. To enable the secure identification of TCSNMP expressing cells to narrow down the potential functions of this high number of TcSNMPs, I needed to establish a reliable and robust double fluorescence in situ hybridization (FISH) protocol for antennae of adult $T$. castaneum.

In order to find T. castaneum specific odorants that need a given TcSNMP for their detection, I wanted to perform loss-of-function studies via RNA interference with subsequent electroantennography measurements (EAG). In contrast to $D$. melanogaster, where only the aggregation pheromone $\mathrm{cVA}$ is characterized as social odor, more described social odors are available in T. castaneum, such as the aggregation pheromone 4-8-dimethyldecanal (DMD) (Kim et al., 2005) or 1-4benzoquinone as a defensive secretion component (Unruh et al., 1998). This gave the great opportunity to analyze the potential involvement of SNMPs to social odors, in addition to their role in pheromone detection.

To analyze, whether the observed conservation of SNMP1 function in $D$. melanogaster and moths is generally true for SNMPs, I wanted to develop an expression system to functionally analyze TCSNMPS and the body specific DmSNMP2 in DmSNMP1-deficient D. melanogaster. Subsequently, the functionality of the fly pheromone system was to be tested, with regard to a potential rescue of the snmp1 mutant situation, in order to identify conserved or adapted functions of 
Introduction

TcSNMPs. To conclude, the functional analyses of the diversified beetle SNMPs were performed to reveal new insights into the possible functions of SNMPs, a protein class that plays a crucial role for the detection of pheromones in $D$. melanogaster. 


\section{$3 \quad$ Material and Methods}

\subsection{Insect culture}

\subsubsection{Tribolium castaneum culture}

All experiments described in this study were carried out with beetles of the vermilion white strain $\left(v^{w}\right)$ (Lorenzen et al., 2002). They were kept at $28^{\circ} \mathrm{C}$ with $40 \%$ humidity on full grain flour with $5 \%$ dry yeast.

\subsubsection{Drosophila melanogaster culture}

D. melanogaster used in this study were raised at $25^{\circ} \mathrm{C}$ on standard cornmeal diet. $5 \mathrm{l}$ of food medium contained following components: $51 \mathrm{~g}$ agar agar strands dissolved in 2,5 $\mathrm{l}$ of demineralized water (dem. $\mathrm{H}_{2} \mathrm{O}$ ), $50 \mathrm{~g}$ soy flour and $90 \mathrm{~g}$ yeast dissolved in 0,5 $\mathrm{l}$ dem. $\mathrm{H}_{2} \mathrm{O}, 400 \mathrm{~g}$ corn flour dissolved in $1 \mathrm{l}$ dem. $\mathrm{H}_{2} \mathrm{O}, 110 \mathrm{~g}$ treacle dissolved in 0,5 $\mathrm{l}$ dem. $\mathrm{H}_{2} \mathrm{O}, 400 \mathrm{~g}$ malt dissolved in 0,5 $\mathrm{l}$ dem. $\mathrm{H}_{2} \mathrm{O}, 31,5 \mathrm{ml}$ propionic acid and 7,5 g nipagin dissolved in $40 \mathrm{ml}$ ethanol. Stocks were kept at $18^{\circ} \mathrm{C}$. In general, fly work was carried out as described in (Greenspan, 1997). Genotypes of the used flies are listed in tables 2-4.

\subsection{Molecular biology}

Unless otherwise noted, kits and reagents were used according to manufacturer`s manuals. Protocols from Sambrook and Russel were used for standard methods and solutions (Sambrook and Russell, 2001). 


\subsubsection{Total RNA extraction}

For total RNA extraction from insect antennae the "ZR Tissue \& Insect RNA MicroPrep $^{\text {TM" }}$ (Zymo Research, Freiburg, Germany) was used. Severed antennae were immediately collected in a ZR BashingBead ${ }^{\mathrm{TM}}$ Lysis Tube filled with ice cold RNA lysis buffer. Subsequently, the manufacturer`s protocol was used. After extraction, the final concentration of total RNA was determined by using the "NanoDrop ND-1000" (Thermo Fisher Scientific Germany BV \& Co KG, Braunschweig, Germany) and provided NanoDrop software.

\subsection{2 cDNA synthesis}

For the double strand cDNA synthesis from antennal total RNA of T. castaneum, the "SMARTer® PCR cDNA Synthesis Kit" (Takara Bio Europe/Clontech, SaintGermain-en-Laye, France) was used. For synthesis of D. melanogaster first strand cDNA, the "Maxima ${ }^{\circledR}$ First Strand cDNA Synthesis Kit" (Thermo Fisher Scientific Germany BV \& Co KG, Braunschweig, Germany) was used.

\subsubsection{RACE (rapid amplification of cDNA ends) cDNA synthesis}

5' and 3' RACE first strand cDNA pools were generated using the "SMARTer" RACE cDNA Amplification Kit" (Takara Bio Europe/Clontech, Saint-Germain-enLaye, France) following the manufacturer's manual.

\subsubsection{Oligonucleotides}

Oligonucleotides that were used in this study were synthesized by Eurofins MWG Operon (Ebersberg, Germany). Oligonucleotide sequences are listed in the following table1. 


\begin{tabular}{|c|c|}
\hline name & $5^{\prime}$ to $3^{\prime}$ sequence \\
\hline RACE_TC008209_S & AAGTTCTGAGGGGCGTCAAGGAATC \\
\hline RACE_TC008209_as & GGCAAGACCTTCCTCCTTTGTGAGA \\
\hline RACE_TC008210b_f & CCAGCACCCGAAAAGTGCCTACCTA \\
\hline RACE_TC008210b_r & CCCAACATTGGAGTTCCGGTTAAAGG \\
\hline RACE_TC008210c_f & ATGCCGATTTCGTTGGTCCTGCTAC \\
\hline RACE_TC008210c_r & TGACCCCGCGAATCAGACTTTCAT \\
\hline RACE_TC008210d_f & GTGGCACGAATTGGGTCGTTTAGTTG \\
\hline RACE_TC008210d_r & CGGGAACTCCGTGAAAACTCGTCTC \\
\hline RACE_TC010353_S & ACCGTGAAGAGAGGTCTCACCAACA \\
\hline RACE_TC010353_as & CTGCAGATGTCCGTCGAATAGATG \\
\hline RACE_TC008191_S & ATTCAAGGAACCGACCTCACCCTCA \\
\hline RACE_TC008191_as & GGTGTACATCCCGGCACCTCATTTT \\
\hline RACE_TC010356_S & GCTGTTGCTGTGTGTACATCATGTTTG \\
\hline RACE_TC010356_1r & GGGTAAGAGGGCGGCCATGTAAATC \\
\hline RACE_TC010356_2f & CTGCGAAAATCGCAACACTGACTCC \\
\hline RACE_TC010356_2r & CTCGTAACCGTCTCGGGTCAGAAGG \\
\hline RACE_356_s3 & GCAATGATCAATCTGTTCCTTGTAATAG \\
\hline RACE_356_s4 & CATGAGCAGTAATTTTATGCGAGTG \\
\hline RACE_356_as4 & CAGAGTCCTTTCCAACTCCAGG \\
\hline RACE_356_s5 & CCATTTCACCAAATGACTGGAGC \\
\hline RACE_356_as5 & GTACAAAGTGGCATCTGTGCCTTG \\
\hline RACE_UPM_short & CTAATACGACTCACTATAGGGC \\
\hline RACE_UPM_long & CTAATACGACTCACTATAGGGCAAGCAGTGGTATCAACGCAGAGT \\
\hline TC008191_start_s & CCTACGTTGATTTAACACTAGCACGC \\
\hline TC008191_end_as & CCTATCTAGGTCACACCTTATGTAAAGTAAGTGCT \\
\hline 353_start2 & GGGGAGGCGCAAAATG \\
\hline 8191_start2 & GCAAAATGCGCCTAAGTACG \\
\hline 8191_end2 & CTCTATTCCACGATTTTCCAAGTG \\
\hline TC010353_end_as & CAAATATATGATATTAATTTGTGCGCACCTATTA \\
\hline 353_end2 & CTATTATTATACATGGTGTTTAGCGTGTGAAC \\
\hline TC008210b_start_s & GTAAGCTTGCAACTTAGTATTGTGAAGTTATTTTAAAAC \\
\hline TC008210b_end_as & AAGTATTCTCAAAAATACAATAATTGTGCCAAACTAC \\
\hline
\end{tabular}




\begin{tabular}{|c|c|}
\hline TC008210c_start_s & ACATGGGGAGTGCAAT \\
\hline TC008210c_end_as & CCATCATTTGATTTAATTAAATAAA \\
\hline TC008210d_start_s & ACATGGGGCACTGTGGCC \\
\hline TC008210d_end_as & CAGTGATTCTACTAAGTGTGTTGAAATTACG \\
\hline 210d_start2 & CCATGCACAAGTCGAAGAAAATACTAG \\
\hline 210d_end2 & CGTTAAAATTTTCCAATTTTTTCGTC \\
\hline SNMP1a_start2 & GAATGAAACCGATTCGTCGG \\
\hline TC008209_end_as & CTAGAACAGAACCTGCCTAAACAAATC \\
\hline SNMP1a_end2 & CATCAGTATTTCTCтAтстCGTTAтCAGAC \\
\hline TC_356_start_s & ATGTCTCGAAAATCTGGAACGGTAC \\
\hline TC_356_end_as & TTATGAACTATTAGCTCTCTCAATGATCCCTTG \\
\hline TcSNMP1aExp_f & GTAAGATCTCAAAATGAAACCGATTCGTCGG \\
\hline TcSNMP1aExp_r & GTAGCGGCCGCTCAGTATTTCTCTATCTCGTTATCAGAC \\
\hline TcSNMP1cExp_f & GTAAGATCTCAAAATGCTTTCTTACAAGAAGATAACTATTATATC \\
\hline TcSNMP1cExp_r & GTAGCGGCCGCTTATTTTGAGCTAAGTACAATATTTTCC \\
\hline TcSNMP2Exp_f & GTAAGATCTCAAAATGGGGAGGCGCAAAATG \\
\hline TcSNMP2Exp_r & GTAGCGGCCGCTTATACATGGTGTTTAGCGTGTGAAC \\
\hline TcSNMP1bExp_f & GTAAGATCTCAAAATGACCACTTGCTTTAACACAAA \\
\hline TcSNMP1bExp_r & GTAGCGGCCGCTTAAGCATTTTTGGTATTTTCTTTATTTC \\
\hline DmSNMP1Exp_f & GTAAGATCTCAAAATGCAAGTACCTCGGGTTAAGCTG \\
\hline DmSNMP1Exp_r & GTAGCGGCCGCTTAGTAGCGCTCCCGATGCTC \\
\hline TcSNMP1dExp_f & GTAAGATCTCAAAATGCACAAGTCGAAGAAAATACTAG \\
\hline TcSNMP1dExp_r & GTAGCGGCCGCTTAAAATTTTCCAATTTTTTCGTC \\
\hline Tc008191Exp_f & GTAAGATCTCAAAATGCGCCTAAGTACGCCC \\
\hline Tc008191Exp_r & GTAGCGGCCGCCTATTCCACGATTTTCCAAGTGG \\
\hline pUASTattb_fw2 & GAATAGGGAATTGGGAATTCGTTAAC \\
\hline pUASTRV & GGTCTTCTACCTTTCTCTTC \\
\hline DmSNMP1_BamHI_f & GTAGGATCCCAAAATGCAAGTACCTCGGGTTAAGCT \\
\hline TcSNMP1a_Seq & CAGCTGAAAGAAGCACCAGACTTG \\
\hline pUASTattbhsp70_s & GTATAAATAGAGGCGCTTCGTCTAC \\
\hline DmSNMP1_800s & CACTTAAGGCGCCAGCTAATGAC \\
\hline DmSNMP1_1200s & GAGAAATTAAGCAGGCCAAACAAGTC \\
\hline Tc210b_1121s & GTGGCCTGAACCAGAATGTAACG \\
\hline Tc210b_820_f & СТСТCACTTACAACCCTTACGACAC \\
\hline
\end{tabular}




\begin{tabular}{|l|l|}
\hline DmSNMP2_BamHI_f & GTAGGATCCCAAAATGATCCACTGGTCTCTGATTGTCAG \\
\hline DmSNMP2_NotI_r & GTAGCGGCCGCTTACTTCACGGGCGGCTC \\
\hline DmSNMP2_r & TTACTTCACGGGCGGCTC \\
\hline T7 & GAATTGTAATACGACTCACTATAGG \\
\hline T7-SP6 & TAATACGACTCACTATAGGATTTAGGTGACACTATAGA \\
\hline
\end{tabular}

Table 1 Oligonucleotides used in this study

\subsubsection{Polymerase chain reaction (PCR)}

Polymerase Chain Reaction (PCR) was used to amplify specific nucleotide sequences, e.g. 5' or 3' cDNA ends, complete open reading frames (ORFs) or templates for dsRNA synthesis. Depending on the kind of desired amplificat and experiment, different polymerases were used. The "Phusion® High Fidelity DNA Polymerase" (New England Biolabs GmbH, Frankfurt am Main, Germany) was used for fast sequence verification of recombinant plasmids. For RACE-PCR and for amplification of full length sequences, the "Advantage ${ }^{\circledR} 2$ PCR Kit" (Takara Bio Europe/Clontech, Saint-Germain-en-Laye, France) was applied. In both cases PCR was performed according to manufacturer`s instructions. PCRs were conducted in the PCR cycler "Eppendorf Mastercycler personal" (Eppendorf, Hamburg, Germany).

\subsubsection{Cloning}

Amplificated DNA was analyzed by agarose gel electrophoresis with regard to its size and quality. DNA bands of the desired size were cut out from the $1 \%$ agarose gel with a clean scalpel and extracted with the "NucleoSpin Gel and PCR Clean-up Kit" (MACHEREY-NAGEL GmbH \& Co. KG, Düren, Germany). Subsequently, the extracted and purified PCR product was ligated into the pCRII vector using the "TA Cloning® Kit Dual Promoter (pCR®II)" (Invitrogen/Life Technologies GmbH, Darmstadt, Germany). The ligation product was transformed into chemically competent Escherichia coli DH5 $\alpha$. The transformed bacteria cells were plated on LB agar plates containing ampicillin as selective agent. Prior to plating of the bacteria solution, $50 \mu \mathrm{L} 4 \% \mathrm{X}$-Gal solution was plated on the LB-Ampicillin agar 
plate to allow blue-white-screening of the recombinant colonies as described in the manual of the pCRII vector.

\subsubsection{DNA isolation of recombinant plasmids}

Isolation of recombinant plasmids was achieved by using the "NucleoSpin $®$ Plasmid" system (MACHEREY-NAGEL GmbH \& Co. KG, Düren, Germany) as described in the manufacturer's protocol. If higher plasmid amounts and/or concentrations were needed, the "Plasmid Midi Kit" (QIAGEN GmbH, Hilden, Germany) was used. The concentration and purity of the plasmid solution was determined using the spectrophotometer NanoDrop ND-1000 (Thermo Fisher Scientific Germany BV \& Co KG, Braunschweig, Germany).

\subsubsection{Double-stranded RNA (dsRNA) synthesis}

The recombinant vector pCRII containing the gene of interest served as template for a PCR using primers with an attached T7 polymerase promoter sequence. The resulting PCR product was used as template in the following in vitro transcription. For the synthesis of dsRNA, the "Ambion $®$ MEGAscript $®$ T7 Kit" (Invitrogen/Life Technologies GmbH, Darmstadt, Germany) was used according to the manufacturer`s protocol. Finally, the precipitated and dried dsRNA was dissolved in $30 \mu$ injection buffer $\left(1.4 \mathrm{mM} \mathrm{NaCl}, 0.07 \mathrm{mM} \mathrm{Na}_{2} \mathrm{H} \mathrm{PO}_{4}, 0.03 \mathrm{mM} \mathrm{KH}_{2} \mathrm{PO}_{4}, 4 \mathrm{mM}\right.$ $\mathrm{KCL}$ ). The dsRNA was stored at $-20^{\circ} \mathrm{C}$. Gel electrophoreses using a $1 \%$ agarose gel was performed to determine the quality and size of the dsRNA. Furthermore, concentration was measured using the "NanoDrop ND-1000" (Thermo Fisher Scientific Germany BV \& Co KG, Braunschweig, Germany) with the provided NanoDrop software. 


\subsection{Sequence analysis and bioinformatics}

\subsubsection{DNA sequence analysis}

As a preliminary test of the identity of isolated plasmid DNA, restriction analysis using appropriate restriction enzymes (New England Biolabs GmbH, Frankfurt am Main, Germany) was performed. Subsequent gel electrophoresis with a $1 \%$ agarose gel allowed detailed restriction fragment analysis. If this indicated that a plasmid contained the desired insert, the plasmid was sent for sequencing to Macrogen (Seoul, Korea and Amsterdam, Netherlands) or LGC genomics (Berlin, Germany). Sequencing was carried out by these companies using Sanger sequencing (Sanger and Coulson, 1975). The obtained sequences were manually checked using "Chromas Lite 2.1" (Technelysium Pty Ltd, South Brisbane, Australia) and analyzed using "BLAST" (Basic Local Alignment Search Tool) provided by NCBI (http://blast.ncbi.nlm.nih.gov/Blast.cgi) as well as "Tribolium BLAST" (http://bioinf.uni-greifswald.de/blast/tribolium/blast.php).

\subsubsection{Sequence alignments}

Sequence alignments of DNA- or protein-sequences for comparative sequence analyses were performed by using Geneious ${ }^{\circledR}$ 6.1.7 (Biomatters Ltd, New Zealand), "BioEdit" (Hall, T.A., 1999), or "MEGA" version 5 (Tamura et al., 2011). The used multiple sequence alignment method was "Clustal W" (Thompson et al., 1994).

\subsubsection{Phylogenetic calculations}

All phylogenetic calculations were performed using MEGA version 5 (Tamura et al., 2011). To construct a phylogenetic tree, full-length amino acid sequences were aligned using the ClustalW algorithm (Thompson et al., 1994). Based on these alignments, phylogenetic calculations were done using the neighbor-joining method (Saitou and Nei, 1987) with the following parameter set: 1000 bootstrap replications, p-distance model, pairwise deletion. 


\subsubsection{Statistical analysis}

All obtained functional data of this study were statistically analyzed with “OriginLab® Origin 8.5” (Origin Lab, Friedrichsdorf, Germany). To test for normality the Kolmogorov-Smirnov test was applied. If this showed that the data were normally distributed, one-way analysis of variance (one-way ANOVA) with posthoc bonferroni pairwise comparison was conducted to compare the respective mean values.

\subsection{Histology}

\subsubsection{Probe synthesis}

Synthesis of digoxigenin (DIG) or biotin-labelled RNA probes was conducted using the respective "RNA Labeling Kit (SP6/T7)" (Roche Diagnostics Deutschland $\mathrm{GmbH}$, Mannheim, Germany) with the following modified protocol: $2 \mu \mathrm{g}$ of linearized recombinant pCRII vector carrying the gene of interest served as template. To this template, $2 \mu \mathrm{l}$ buffer, $2 \mu \mathrm{l}$ respective RNA labeling mix and $2 \mu \mathrm{l}$ SP6 or T7 RNA Polymerase was added. SP6 or T7 RNA Polymerase was used according to the orientation of the insert and whether a sense or antisense probe was desired to be synthesized. For synthesis of an antisense probe the polymerase that starts at the $5^{\prime}$ side towards the $3^{\prime}$ end of the antisense strain was used. Finally, autoclaved MilliQ water was added to a final volume of $20 \mu$ l. After an incubation time of $3 \mathrm{~h}, 1 \mu \mathrm{l}$ DNaseI (Roche Diagnostics Deutschland GmbH, Mannheim, Deutschland) was added to digest the DNA template. After adding $2.5 \mu$ lithium chloride (5 M) and $75 \mu \mathrm{l} 100 \%$ ethanol, the RNA probe was precipitated via incubation at $-80^{\circ} \mathrm{C}$ for $30 \mathrm{~min}$ and subsequent centrifugation at $4^{\circ} \mathrm{C}$ for $30 \mathrm{~min}$. The obtained RNA probe pellet was washed with $70 \%$ ethanol followed by a second centrifugation step. The dried pellet was dissolved in $50 \mu \mathrm{l}$ water. 


\subsubsection{Probe fragmentation}

By incubation in carbonate buffer ( $80 \mathrm{mM} \mathrm{NaHCO}_{3}, 120 \mathrm{mM} \mathrm{Na}_{2} \mathrm{CO}_{3}, \mathrm{pH} 10.2$ ) all RNA probes were fragmented to an average length of about $200 \mathrm{bp}$ following the protocol of Angerer \& Angerer (Angerer LM, Angerer RC., 1992). Fragmented probes were stored at $-20^{\circ} \mathrm{C}$ in a buffer containing $50 \%$ formamide, $10 \%$ dextran sulfate, $0,2 \mu \mathrm{g} / \mu \mathrm{l}$ yeast tRNA, $0,2 \mu \mathrm{g} / \mu \mathrm{l}$ sonicated salmon sperm DNA and $2 \mathrm{x}$ SSC.

\subsubsection{Fluorescence in situ hybridization (FISH)}

Fluorescence in situ hybridization (FISH) on T. castaneum antennae was performed as described for Anopheles gambiae antennae (Schultze et al., 2012) with several modifications: Unless otherwise noted, all steps were performed in $1.5 \mathrm{ml}$ Eppendorf tubes at room temperature and all used buffers contained 0,03\% Triton X-100 to avoid sticking of the antennae. Beetles were anesthetized on ice before the antennae were severed with forceps. Collected antennae were transferred into ice cold fixation solution (4\% paraformaldehyde in $0.1 \mathrm{M} \mathrm{NaCO}_{3}$, pH 9.5, $0.03 \%$ Triton X-100) and incubated for $24 \mathrm{~h}$ at $4^{\circ} \mathrm{C}$. The fixated tissue was then put into a silicon form to become embedded in tissue freezing medium (“Tissue-Tek® O.C.T. Compound”, Science Services GmbH, München, Germany). To freeze the embedded sample, it was kept at $-20^{\circ} \mathrm{C}$ for $10 \mathrm{~min}$. When completely frozen, the cryotome "Cryostat CM 1950" (Leica, Nussloch, Germany) was used to bisect the antennae by cutting them to slices of a thickness of $50 \mu \mathrm{m}$. Subsequently, the frozen slices were collected in a cold Eppendorf tube. $1000 \mu \mathrm{l}$ PBS were added to melt the tissue freezing medium. After removal of the melted freezing medium, the antennae were washed for $1 \mathrm{~min}$ in PBS, followed by a 10 min washing step in $0.2 \mathrm{M} \mathrm{HCl}$ and 1 min incubation in PBS $+1 \%$ Triton X-100.

Afterwards, the antennae were kept in the hybridization solution (50\% formamide, 5x SSC, $1 \mathrm{x}$ denhardt's reagent, $50 \mu \mathrm{g} / \mathrm{ml}$ yeast RNA, $1 \%$ Tween 20, $0.1 \%$ Chaps, $5 \mathrm{mM}$ EDTA, pH 8.0) for 1 to 10 days at $4^{\circ} \mathrm{C}$. Prehybridization was achieved by heating the antenna to $55^{\circ} \mathrm{C}$ for $5 \mathrm{~h}$ before adding the desired probes. Probes were diluted 1:50 or 1:100 (depending on the expression level of the transcript that had to be labelled) in hybridization solution (hyb). To ensure that 
the probe is single stranded the probe/hyb solution was heated to $65^{\circ} \mathrm{C}$ for $5 \mathrm{~min}$. Subsequently, the probe/hyb solution was chilled on ice for $10 \mathrm{~min}$ before it was added to the antennae.

After probe incubation for 3 days at $55^{\circ} \mathrm{C}$, the antennae were washed four times for $15 \mathrm{~min}$ each in $0,1 \mathrm{x}$ SSC at $60^{\circ} \mathrm{C}$. In order to block unspecific binding sites for the subsequent antibody incubation the antennae were treated with "Blocking reagent" (Roche Diagnostics Deutschland GmbH, Mannheim, Germany) for $5 \mathrm{~h}$ at $4^{\circ} \mathrm{C}$. For the detection of DIG-labelled probes, Fab fragments of anti-digoxigenin-AP antibodies (Roche Diagnostics Deutschland GmbH, Mannheim, Germany) were used, diluted 1:500 in blocking reagent. To detect biotin-labelled probes, a Streptavidin-HRP conjugate (PerkinElmer, Rodgau, Germany) was added to the blocking reagent. In case of a desired nuclear staining of the samples, DAPI was added in a dilution of $1: 1000$. After 3 days of incubation at $4^{\circ} \mathrm{C}$, the antennae were washed $5 \times 10$ min with TBS $+0.05 \%$ Tween 20 . To visualize transcripts labelled with DIG-probes, the "HNPP Fluorescent Detection Set" (Roche Diagnostics Deutschland GmbH, Mannheim, Germany) was used. The incubation time was $1 \mathrm{~h}$ to $3 \mathrm{~h}$ at room temperature, depending on the applied probe. Biotin-labelled probes were visualized by using the "TSA", Fluorescein System" or the "TSA ${ }^{\mathrm{TM}}$ Plus Fluorescein System" (PerkinElmer, Rodgau, Germany) after washing the respective samples three times for $5 \mathrm{~min}$ each in TBS. The incubation time was $3 \mathrm{~h}$ at room temperature.

Finally, antennae were washed three times for 5 min each in TBS and transferred to PBS before they were embedded in "Mowiol@ 4-88" (Sigma-Aldrich Chemie $\mathrm{GmbH}$, München, Germany). The embedded samples were stored at $-20^{\circ} \mathrm{C}$ and analyzed via microscopy (chapter 3.6.2).

\subsection{Genetics}

\subsubsection{RNA interference (RNAi)}

In order to knock-down a transcript of interest, RNA interference (RNAi) was performed. Therefore, T. castaneum pupae were injected with dsRNA (2000 ng/ $\mu \mathrm{l})$ 
of full-length ORF sequences of the desired gene. Injections were performed as already described in Posnien et al. (2009). After hatching, the beetles were checked for physical integrity, visible by normal vitality and absence of lacerations.

\subsubsection{D. melanogaster transgenesis}

Transgenesis was performed in D. melanogaster to create UAS-responder lines for functional rescue experiments.

\subsubsection{Cloning of UAS-constructs}

To enable GAL4/UAS-mediated expression of transgenes, the gene of interest had to be cloned into the pUASTattB vector (Bischof et al., 2007). In a first step, the genes of interest were amplified by PCR using a forward primer with an attached translation start consensus sequence (Cavener, 1987). Furthermore, the forward as well as the reverse primer were designed to carry restriction sites. The PCR product was subcloned into the pCRII vector (as described in chapters 3.2.6 and 3.2.7) and its identity was verified by sequencing (as described in chapter 3.3.1). Subsequently, the sequenced plasmids, carrying the gene of interest, as well as the pUASTattB vector were digested with corresponding restriction enzymes (New England Biolabs GmbH, Frankfurt am Main, Germany) and purified by gel electrophoresis and the "NucleoSpin Gel and PCR Clean-up Kit" (MACHEREYNAGEL GmbH \& Co. KG, Düren, Germany). After ligation of the desired, purified DNA sequences of the gene of interest in the pUASTattb with the T4 DNA Ligase (5 U/ $\mu \mathrm{L}$; Invitrogen/Life Technologies GmbH, Darmstadt, Germany), the UASconstructs were transformed into chemically competent Escherichia coli DH5 $\alpha$ as described in chapter 3.2.6. Afterwards, a Midi-preparation was conducted by using the "Plasmid Midi Kit” (QIAGEN GmbH, Hilden, Germany; chapter 3.2.7).

\subsubsection{Generation of transgenic D. melanogaster UAS-lines}

All UAS-lines used in this study were generated by Best Gene Inc. (Chino Hills, USA). The UAS-constructs were injected in Basler strain (FlyC31 strain) \#24482. 
After arrival, single male transformants were balanced by crossing with virgins of a balancer line (see table 2). Finally, the balanced stock was kept at $18^{\circ} \mathrm{C}$.

\begin{tabular}{|l|l|l|}
\hline genotype & source/publication & type \\
\hline $\mathrm{w} 1118 ; \mathrm{CyO} / \mathrm{Sp} ; \mathrm{TM} 2 / \mathrm{MKRS}$ & $\begin{array}{l}\text { wr135 stock collection } \\
\text { (Lindsley and Zimm, 2012) }\end{array}$ & balancer line \\
\hline \multirow{2}{*}[^{*}]{$; \mathrm{Bl} / \mathrm{Cyo} ; \mathrm{TM} 2 / \mathrm{TM} 6 \mathrm{~B}$} & $\begin{array}{l}\text { provided by Dept. of } \\
\text { Developmental Biochemistry, } \\
\text { University Göttingen }\end{array}$ & balancer line \\
\hline
\end{tabular}

Table 2: D. melanogaster balancer lines used in this study

\subsubsection{Generation of $D$. melanogaster lines used for functional analyses}

The D. melanogaster initial lines (table 3) were used to generate the parent stocks (table 4) of the flies measured in the functional analyses. Genetic crossings and homologous recombination of flies were performed as described in (Greenspan, 1997).

\begin{tabular}{|c|c|c|}
\hline genotype & source/publication & type \\
\hline $\mathrm{w}\left[{ }^{*}\right] ;$ SNMP-Gal4/CyO & $\begin{array}{l}\text { provided by Leslie Vosshall } \\
\text { (Benton et al., 2007) }\end{array}$ & driver line \\
\hline $\mathrm{w}\left[{ }^{*}\right] ; \mathrm{Bl}[1] / \mathrm{CyO} ; \operatorname{snmp} 1[2] / \mathrm{TM} 6 \mathrm{~B}$ & Bloomington \#25042 & mutant line \\
\hline w[*]; bw/bw; st, vainsD/TM6B & $\begin{array}{l}\text { provided by Dean Smith } \\
\text { (Jin et al., 2008) }\end{array}$ & mutant line \\
\hline w1118; P $\{20 X U A S-I V S-G C a M P 5 G\} a t t P 40$ & Bloomington \#42037 & UAS line \\
\hline w[*]; UAS-TcSNMP1a/Cy0; TM2/TM6B & this study & UAS line \\
\hline w[*]; UAS-TcSNMP1b.1/CyO; TM2/TM6B & this study & UAS line \\
\hline w["]; UAS-TcSNMP1b.2/Cy0; TM2/TM6B & this study & UAS line \\
\hline w[*]; UAS-TcSNMP1c/Cy0; TM2/TM6B, & this study & UAS line \\
\hline w[*]; UAS-TcSNMP1d/Cy0; TM2/TM6B & this study & UAS line \\
\hline w[*]; UAS-Tc008191/CyO; TM2/TM6B & this study & UAS line \\
\hline w[*]; UAS-TcSNMP2/CyO; TM2/TM6B & this study & UAS line \\
\hline $\mathrm{w}\left[^{*}\right] ;$ UAS-DmSNMP1/CyO; TM2/TM6B & this study & UAS line \\
\hline w["]; UAS-DmSNMP2.B/Cy0; TM2/TM6B & this study & UAS line \\
\hline
\end{tabular}




\begin{tabular}{|l|l|l|}
\hline w[*]; UAS-DmSNMP2.C/Cy0; TM2/TM6B & this study & UAS line \\
\hline
\end{tabular}

Table 3: D. melanogaster initial lines used in this study

\begin{tabular}{|c|c|}
\hline genotype & type \\
\hline w[*]; SNMP-Gal4, UAS-GCaMP5G/CyO; vainsD/TM6B & driver line \\
\hline $\mathrm{w}{ }^{*}$ ]; SNMP-Gal4, UAS-GCaMP5G/CyO; +/TM6B & wild type control \\
\hline w[*]; UAS-TcSnmp1a/Cy0; snmp1[2]/TM6B & UAS line \\
\hline w[*]; UAS-TcSNMP1b.1/Cy0; snmp1[2]/TM6B & UAS line \\
\hline w[*]; UAS-TcSNMP1b.2/CyO; snmp1[2]/TM6B & UAS line \\
\hline $\mathrm{w}\left[{ }^{*}\right] ;$ UAS-TcSnmp1c/Cy0; snmp1[2]/TM6B, & UAS line \\
\hline w[*]; UAS-TcSNMP1d/Cy0; snmp1[2]/TM6B & UAS line \\
\hline $\left.\mathrm{w}{ }^{*}\right] ;$ UAS-Tc008191/CyO; snmp1[2]/TM6B & UAS line \\
\hline w[*]; UAS-TcSnmp2/CyO; snmp1[2]/TM6B & UAS line \\
\hline $\mathrm{w}\left[{ }^{*}\right] ;$ UAS-DmSnmp1/Cy0; snmp1[2]/TM6B & UAS line \\
\hline w[*]; UAS-DmSNMP2.B/Cy0; snmp1[2]/TM6B & UAS line \\
\hline w[*]: UAS-DmSNMP2.C/CyO; snmp1[2]/TM6B & UAS line \\
\hline
\end{tabular}

Table 4: D. melanogaster parental lines of flies used for functional analysis in this study

For functional analyses virgins of the driver line

w[*]; SNMP-Gal4, UAS-GCaMP5G/Cy0; vainsD/TM6B (table 4) were crossed with males of the desired UAS-line (UAS-X) (table 4). The offspring was screened for their genotype. Flies with the genotype

$$
\text { w[*]; SNMP-Gal4, UAS-GCaMP5G/UAS-X; vainsD/ snmp1[2] }
$$

were measured as UAS-X rescue fly, whereas the siblings with the following genotype

$$
\text { w[*]; SNMP-Gal4, UAS-GCaMP5G/Cy0; vainsD/ snmp1[2] }
$$

were measured as snmp1 mutant control fly. 


\subsection{Imaging}

\subsection{1 in vivo two-photon calcium imaging of D. melanogaster}

To measure neuronal activity of olfactory sensory neuron (OSNs) in the antennal lobe, calcium imaging was performed as described in (Barth et al., 2014) with the following modifications: 2-7 days old female D. melanogaster, expressing the calcium sensor GCamP5G (Akerboom et al., 2012), were anesthetized on ice. After opening the head capsule, ice cold Ringer's solution (5 mM HEPES, pH 7.4, $130 \mathrm{mM}$ $\mathrm{NaCl}, 5 \mathrm{mM} \mathrm{KCl}, 2 \mathrm{mM} \mathrm{CaCl}$, $2 \mathrm{mM} \mathrm{MgCl}$, $36 \mathrm{mM}$ sucrose) was applied to cover the opening. Measurements were conducted using an "LSM 7 MP two-photon microscope" (Carl Zeiss Microscopy GmbH, Jena, Germany) equipped with a modelocked "Ti-sapphire Chameleon Vision II laser" (Coherent, Dieburg, Germany), a 500-550 nm bandpass filter and a Plan-Apochromat 20×/1.0 NA water-immersion objective (Carl Zeiss Microscopy $\mathrm{GmbH}$, Jena, Germany). A frame rate of $5 \mathrm{~Hz}$ with an excitation wavelength of $920 \mathrm{~nm}$ was used to monitor calcium dynamics. Odors were diluted with mineral oil. Hereby, 1-hexanol and isoamyl acetate (SigmaAldrich Chemie Gmbh, München, Germany) were diluted 1:100 and cVA (Biomol, Hamburg, Germany) was diluted 1: 10. Odorants were applied for $2 \mathrm{sec}$ at a constant flow rate of $1 \mathrm{ml} / \mathrm{s}$ directly to the fly's antennae by a custom-built "olfactometer" device as described by Riemensperger et al. (2005). To correct for slight movements of the preparation, acquired images were aligned in the X-Ydirection using a custom-written Fiji plugin (Schindelin et al., 2012). After determination of the region of interest, the baseline fluorescence $\left(\mathrm{F}_{0}\right)$ was calculated by computing the mean of five images before odor stimulus. This value was subtracted from the fluorescence intensity values during odor application. Finally, the resulting difference value was divided by $\mathrm{F}_{0}$ to get the difference of fluorescence emission $(\Delta \mathrm{F})$. Every fly was measured three times and each $\Delta \mathrm{F}$ was calculated. For subsequent analyses all three $\Delta \mathrm{F}$ were averaged. 
Material and Methods

\subsubsection{Microscopy and image processing}

Embedded T. castaneum antennae that had been subjected to FISH (chapter 3.4.3) were analyzed with a "Zeiss LSM780 laser scanning microscope" (Carl Zeiss Microscopy GmbH, Jena, Germany) using a $405 \mathrm{~nm}, 488 \mathrm{~nm}$, and a $561 \mathrm{~nm}$ laser. Confocal image stacks were taken from single antennal segments. These stacks were projected to a single picture. If desired, several of these were arranged to show coherent antennal stretches using "PowerPoint" (Microsoft, Unterschleißheim, Germany).

\subsection{Electroantennography (EAG) of T. castaneum}

Insect preparation and recording was conducted as described in Wibe et al. (2004) with following modifications: Studied beetles of 7-10 days age were starved for $24 \mathrm{~h}$. Subsequently, the beetle was wedged into a pipette tip, so that the antennae were directed towards the small end of the tip. The tip was cut just above the beetles's head to enable the antennae to come out of the opening. The pipette tip was cut a second time approximately $2 \mathrm{~mm}$ behind the beetle to fill the resulting opening with plasticine material. This prevented the beetle from moving backwards and pushed the antennae softly out of the front tip cut. A sharpened tungsten wire was used to make a small hole at the thorax region to insert the reference glass electrode. This electrode was filled with Ringer solution and contained a silver wire.

The beetle was placed under the high magnification compound microscope "Leica MZ16" (Leica microsystems, Wetzlar, Germany) and the antenna was stabilized using a sharpened tungsten wire, prepared with electrolyte solution. By using a micromanipulator and a sharpened tungsten wire, the last antennal segment was punctured and a sharpened glass capillary electrode (GB150F-8P, $0.86 \times 1.50 \times 80 \mathrm{~mm}$ with filament, Science products $\mathrm{GmbH}$, Hofheim, Germany) filled with Ringer solution, in contact with a silver wire, was inserted.

The DC potential was recorded on a computer using a custom-built amplifier (Universal AC/DC probe), processed with a data acquisition controller "IDAC-4 
A/D" converter and later analyzed using "EAG 2000" software (Syntech, Hilversum, Netherlands). During the EAG recordings the antenna was flushed with a constant flow (approximately $3 \mathrm{l} / \mathrm{min}$ ) of filtered and humidified air. $20 \mu \mathrm{L}$ of the diluted stimulus compound was applied to a $2 \mathrm{~cm}^{2}$ piece of filter paper $(7 \times 40 \mathrm{~mm}$, Whatman No.1) and transferred into a glass Pasteur pipette (Pasteur capillary pipette, 150mm Wu Mainz, Deutschland). For odor application an electrically controlled stimulus controller (CS-02, Syntech, Hilversum, Netherlands) delivered a $1 \mathrm{~s}$ puff of odor to the antenna. To confirm the activity of an antenna preparation, the positive control odors 4-8-dimethyldecanal (DMD) at 10-3 and 1-hexanol at $10^{-2}$ were applied at the beginning and the end of recording. The negative control odor silicon oil was used before and after each replication. The desired test odors were applied in random order. For testing dose dependent responses odors were applied with increasing concentrations. Between measurements a pause of at least one minute was allowed. For every sex, seven beetles were recorded and every beetle was recorded twice. The mean of both measurements was calculated and used as value for this beetle in subsequent analyses. 
Results

\section{$4 \quad$ Results}

\subsection{Sequence analysis of TcSNMPs}

The expression of six TCSNMPs in antennal tissue of adult T. castaneum was confirmed by transcriptome data (Dippel et al. in preparation). However, the originally predicted coding sequences were based on in silico annotations and were corrected during transcriptome-based reannotation. Therefore, the new gene predictions had to be verified and the actual full-length cDNA sequences of the different TCSNMPs had to be determined.

\subsection{1 cDNA isolation and characterization of TcSNMPS}

To determine the actual TCSNMP sequences, 5' and 3' RACE PCRs on antennal cDNA of T. castaneum were conducted. Specific oligonucleotides were designed to prime regions for which the sequence annotation was supported by available transcriptome data (Dippel et al. in preparation). It was possible to obtain $5^{\prime}$ and $3^{\prime}$ transcript sequences of TcSNMP1a, TcSNMP1b, TcSNMP1c, TcSNMP1d, TcSNMP1z, and TCSNMP2. The sequence information of these transcripts was sufficient to assemble the complete ORFs (open reading frame) in silico. Based on this assembly, primer pairs were designed to amplify the full length sequences of all TcSNMPs by PCR on antennal cDNA of T. castaneum, which verified the in silico cDNA assemblies.

The determined TCSNMP1a transcript is 1980 bp (base pairs) long and contains a five bp long 5' UTR and 304 bp long 3' UTR (figure 4.1). Eight exons contain the $1671 \mathrm{bp}$ long ORF which results in 557 amino acids in the corresponding putative protein (figures 4.1 and 4.2). By comparison of the isolated TcSNMP1a cDNA sequence and the originally annotated sequence the actual ORF is $126 \mathrm{bp}$ longer, due to the actual translation start being located $126 \mathrm{bp}$ upstream to the predicted start codon (figure S1). 
For $T c S N M P 1 b$, it was possible to isolate two isoforms of the TcSNMP1b transcript: TcSNMP1b.1 and TcSNMP1b.2 (figure 4.1). Here, due to alternative splicing, the transcript of the isoform TcSNMP1b.1 contains an additional exon that results in a 252 bp longer ORF (figure 4.1). More precisely, TcSNMP1b.1 has a 1587 bp long ORF and TcSNMP1b.2 a 1335 bp long ORF (figure 4.1). After in silico translation this results in 84 additional amino acids in the corresponding putative TcSNMP1b.1 protein (figure 4.2). The determined 5' UTR is 40 bp long and the 3' UTR is $59 \mathrm{bp}$ long (figure 4.1). Compared to the annotation, TcSNMP1b.1 (figure S2) as well as TcSNMP1b.2 (data not shown) have a translation start that is 92 base pairs upstream of the annotated start codon and a 15 bp longer 3'UTR than predicted (figure S2).

The full length TcSNMP1c transcript obtained via RACE-PCR, comprises eight exons containing an ORF of 1461 base pairs, resulting in a putative protein with 487 amino acids (figures 4.1 and 4.2). The 5' UTR of TcSNMP1c has a length of 8 bp and the 3' UTR of $26 \mathrm{bp}$ (figure 4.1). The actual TcSNMP1d transcript is $1686 \mathrm{bp}$ long with an ORF of 1572 bp (figure 4.1). The corresponding putative protein has 524 amino acids (figure 4.2). Here, TCSNMP1C represents the $5^{\prime}$ end of the annotated TcSNMP1C sequence and TcSNMP1d represents the $3^{\prime}$ end (figures S3 and S4). Given that both sequences were isolated by RACE PCR, this confirms the transcriptome based observations that TcSNMP1c and TcSNMP1d are independent transcripts (Dippel et al. in preparation).

The obtained full length cDNA of TcSNMP1z is $1860 \mathrm{bp}$ long containing a $35 \mathrm{bp}$ long 5' UTR and a 241 bp long 3' UTR (figure 4.1). Six exons contain a 1584 bp long ORF which results in a putative protein sequence of 528 amino acids (figures 4.1 and 4.2). This verifies the existence of this TCSNMP gene, which had been only fragmentally detected by bioinformatical tools (figure S5). Therefore, the obtained full length sequence will enable the determination of conserved domains and protein topology of the putative protein.

The isolated TcSNMP2 cDNA sequence has an ORF of 1539 bp resulting in 513 amino acids in the corresponding putative protein (figures 4.1 and 4.2). The full length cDNA of TCSNMP2 consists of nine exons and is $1600 \mathrm{bp}$ long with a $2 \mathrm{bp}$ long 5' UTR and a 59 bp long 3' UTR (figure 4.1). Comparison of the actual TCSNMP2 cDNA sequence with the in silico annotation showed an only marginal 
Results

shortening of the determined ORF at the $5^{\prime}$ end of $14 \mathrm{bp}$. The $3^{\prime} \mathrm{UTR}$ of the annotated transcript is 68 bp longer than the obtained 3'UTR (figure S6).

Generally, comparisons between the isolated full length transcripts with the annotated sequences show several single nucleotide polymorphisms (SNPs) (figures S1-S6). However, the majority of these polymorphisms do not lead to an amino acid exchange. These SNPs could be allelic variations of the same gene within different beetle strains, since different strains were used for the cDNA preparation and for the genome sequencing.

Taken together, it was possible to show that six of the seven originally annotated TCSNMP genes (Nichols and Vogt, 2008; Vogt et al., 2009) actually exist as independent transcripts in antennae of adult T. castaneum, confirming the result of the transcriptome analysis (Dippel et al. in preparation). Importantly, consistent full-length cDNA sequences of these genes were obtained, cloned, and are now available (sequences as fasta-format are listed in the supplemental section). They were used for all subsequent experiments in this work. 


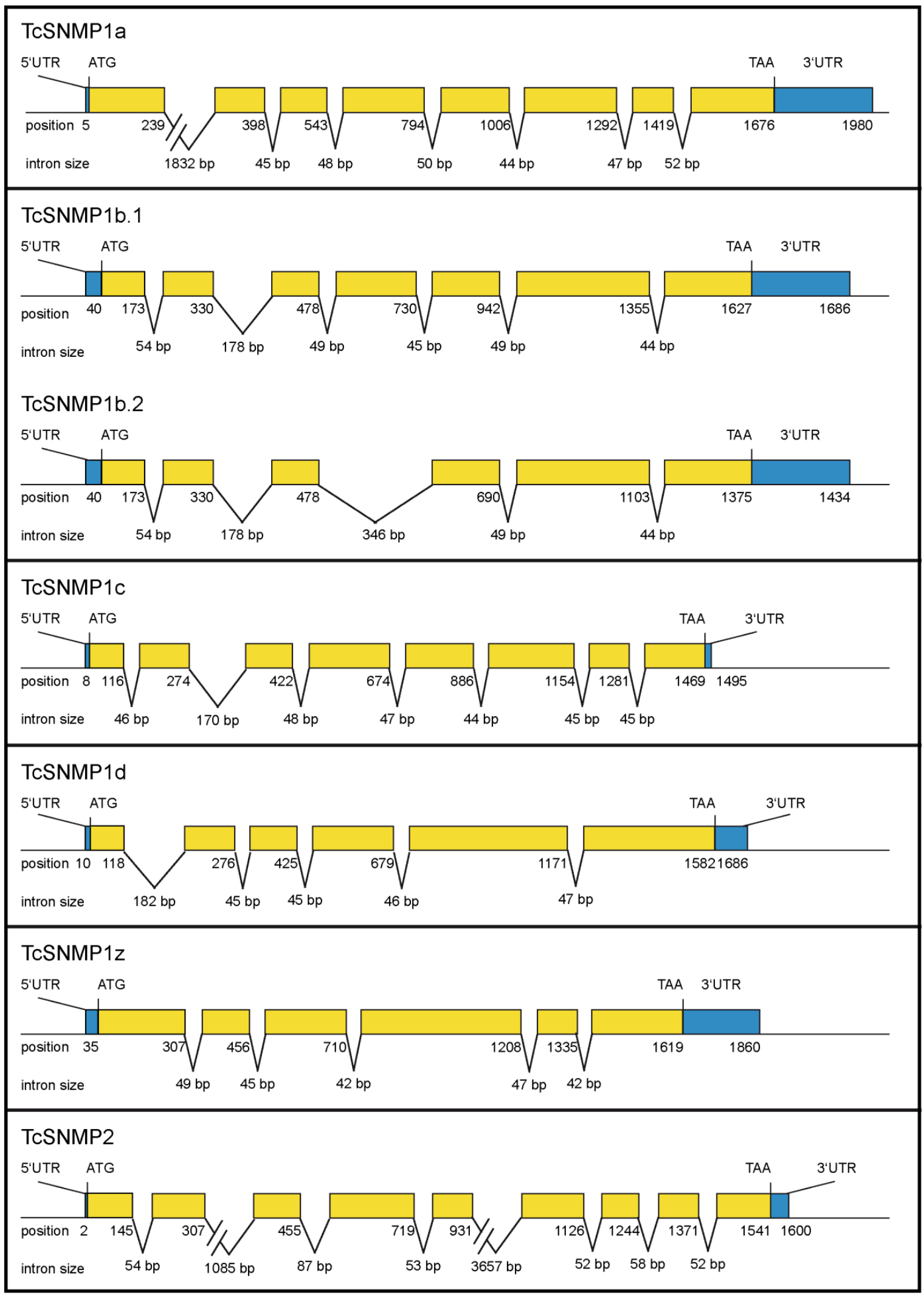

Figure 4.1 Full-length cDNA sequences of the isolated TcSNMPs

The genomic regions (black line) and the actual TcSNMP full-length cDNA sequences obtained by RACE-PCR (boxes) are schematically shown. The ORF is illustrated by yellow boxes and the $5^{\prime}$ and 3' UTR by blue boxes. Numbers below the boxes indicate the nucleotide position of the full-length cDNA sequence. Numbers below the depicted introns indicate their length in bp. The genomic and 
Results

cDNA areas are proportional in size. Exceptions are indicated as interruption of the depicted introns.

\subsubsection{Analysis of the amino acid sequence and topology of TcSNMP proteins}

The obtained ORFs (chapter 4.1.1) were translated in silico, to determine the TcSNMP amino acid sequences. These were used to characterize the TcSNMP proteins and to analyze their topology. By using BLAST algorithm, all putative TcSNMP protein sequences were confirmed as SNMP proteins. Direct comparison of the TcSNMP protein sequences with each other revealed a considerable number of identical amino acid residues (figure 4.2).

However, except of TcSNMP1z, the total sequence identity of two given TcSNMP1 proteins among each other was in the range of $28.3 \%$ to $44.3 \%$ (figure 4.3 ). Here, TcSNMP1a and TcSNMP1b.2 were the two TcSNMPs with the lowest identity of $28.3 \%$, whereas TcSNMP1c and TcSNMP1d showed the highest identity with $44.3 \%$ (figure 4.3 ). In contrast to the T. castaneum SNMP proteins, moth SNMP1 proteins of the tobacco budworm Heliothis virescens, the tobacco hornworm Manduca sexta and the silk moth Antheraea polyphemus showed a much higher sequence identity of $66 \%$ - $90 \%$ (Forstner et al., 2008).

The TcSNMP2 protein revealed a sequence identity of $23 \%$ to $26.2 \%$. with TcSNMP1a, both TcSNMP1b isoforms, TcSNMP1c and TcSNMP1d. Here, $23 \%$ shared amino acids were found with the TcSNMP1b.2 protein and $26.2 \%$ with the TcSNMP1d protein (figure 4.3). Similarly, the TcSNMP1z protein showed a sequence identity of $20.7 \%-24 \%$ to the other TcSNMP1 proteins and $24.5 \%$ to the TcSNMP2 protein (figure 4.3). These values are comparable to the described amino acid identities of 25\%-29\% between SNMP1 and SNMP2 proteins of $H$. virescens, M. sexta and A. polyphemus (Forstner et al., 2008). This low amino acid identity of TcSNMP1z to the other TcSNMP1 proteins and the TcSNMP2 protein indicates a special position of TcSNMP1z within the T. castaneum SNMP proteins.

SNMP proteins belong to the larger CD36 scavenger receptor protein family (Benton et al., 2007; Rogers et al., 1997). Like these, known SNMP proteins have two transmembrane domains (TMD), short intracellular N- and C-termini and a big 
extracellular loop (Martin et al., 2011; Rogers et al., 1997). To test whether the T. castaneum SNMPs also have putative transmembrane domains, the sequences were analyzed by the „TMHMM ExPASy Proteomics tool“ (Möller et al., 2001). In all TcSNMP protein sequences, two TMD were found (red frames, figure 4.2). They were all predicted with a very high probability.

Based on the predicted TMD, all TcSNMP proteins contain one huge extracellular loop. The N- and C-termini of the proteins are relatively small and are exposed to the intracellular compartment. Taken together, all T. castaneum SNMPs possess the typical characteristics and topology of SNMPs and CD36 proteins.

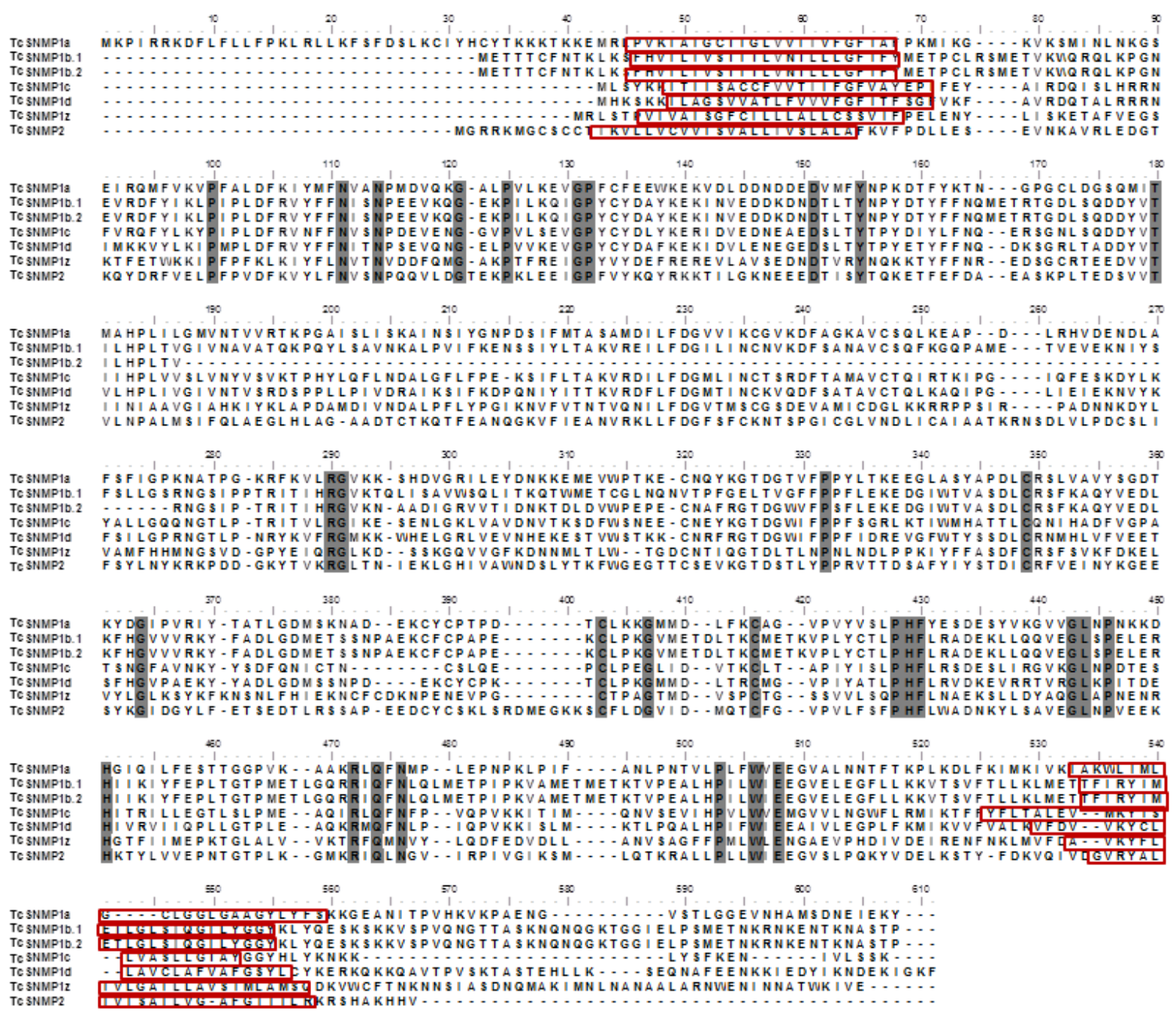

Figure 4.2 Alignment of amino acid sequences of all obtained TcSNMP proteins

Shown are the amino acid sequences of the indicated TcSNMPs in one letter code. Protein alignment was done using ClustalW (Thompson et al., 1994). Identical amino acids are highlighted in dark grey. Putative transmembrane domains were identified via TMHMM prediction tool (Möller et al., 2001) and are framed in red. 


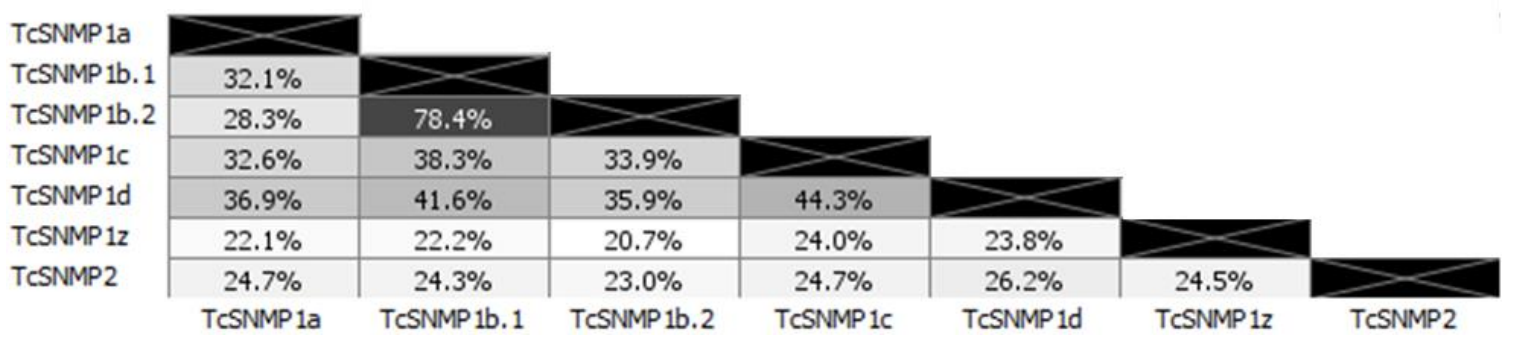

Figure 4.3 Amino acid identities among TcSNMP proteins

Amino acid identities of any two TcSNMP proteins are shown in percent. In addition, the percentages are depicted as a heat map. The darker the grey shade of the heat plot the higher the amino acid identity.

\subsubsection{Phylogenetic analysis of TcSNMP proteins with SNMP proteins and other members of the CD36 protein family}

To determine the degree of relationship between the SNMP proteins of T. castaneum and SNMPs and other CD36-related proteins of other species, a phylogenetic analysis was performed using the MEGA5 software (Tamura et al., 2011). Here, the protein alignment was conducted using ClustalW and the tree was calculated with the neighbor joining algorithm (Saitou and Nei, 1987; Thompson et al., 1994). This was done to clarify the identity of the numerous TcSNMP proteins, especially of the TcSNMP1z protein that showed low amino acid identities to the other TcSNMP proteins (figure 4.3).

In addition to the obtained TcSNMP members, also other protein sequences were included in this phylogenetic analysis. In this respect just protein sequences with a known full length protein sequence were taken. As representatives of the human CD36 protein family, two different well characterized CD36 proteins were included, the human CLA-1 (CD36 and LIMPII analogous-1) and the fatty acid translocase CD36 (Putri et al., 2015; Song et al., 2015). As further members of the mammalian CD36 protein family, the bovine SRB1 (scavenger receptor class B member 1) and the SCRB2 (scavenger receptor class B member 2) of mouse and rat were included. The used CD36 proteins of the vinegar fly D. melanogaster DmelSantaMaria (scavenger receptor acting in neural tissue and majority of rhodopsin is absent), DmelEmp (epithelial membrane protein), DmelNinaD (neither inactivation nor afterpotential D), DmelCrq (croquemort) and 
DmelCG1887 (DmelCG1887/debris buster) are all well described regarding their potential function (Franc et al., 1999; Han et al., 2014; Kiefer et al., 2002; Lemaitre, 2000; Wang et al., 2007). All these non-SNMP CD36 proteins served as outgroup.

SNMP1 and SNMP2 protein sequences were taken from insects that have an available transcriptome to prevent missing SNMP homologs. Furthermore phylogenetic relations of the species were considered. Here, the protein sequences of the SNMP homologs of the honeybee Apis mellifera (AmelSNMPs) as representative of the hymenoptera were taken. Of Diptera, the SNMP homologs of the yellow fever mosquito Aedes aegypti (AaegSNMPs), the malaria mosquito Anopheles gambiae (AgamSNMPs) and the vinegar fly D. melanogaster (DmelSNMPs) were included. For Lepidoptera the SNMP sequences of the tobacco budworm Heliothis virescens (HvirSNMPs), the tobacco hornworm Manduca sexta (MsexSNMPs) and the domesticated silkmoth Bombyx mori (BmorSNMPs) were used. To improve the tree resolution, additional beetle SNMP homologs of the yellow mealworm beetle Tenebrio molitor (TmolSNMPs) were included. Additionally, SNMP protein sequences of the hessian fly Mayetiola destructor (MdesSNMPs) and the brown blowfly Calliphora stygia (CstySNMPs), two insects that have a rather high number of described SNMPs, were included.

The resulting dendrogram (figure 4.4) shows that all SNMP proteins included in the analysis cluster together in one main group. This group subdivides further into a smaller branch that comprises the different SNMP1 proteins and into one that includes the SNMP2 proteins. The only exceptions are Calliphora SNMP3 that clusters in the SNMP1 protein branch and Tribolium SNMP1z that, similar to Apis SNMP2, groups neither into the SNMP1 or SNMP2 proteins. The SNMP1 proteins as well as the SNMP2 proteins, respectively, of the Diptera (figure 4.4 dark grey background) and of the Lepidoptera (figure 4.4 light grey background) cluster in their respective orders.

In contrast, the Tribolium SNMP1 proteins are distributed into two coleopteran branches. The one branch consists of TcSNMP1b, $c$ and $d$ and the other consists of TcSNMP1a and the SNMP1 of the beetle Tenebrio. Tribolium SNMP2 clusters with the SNMP2 protein of the beetle Tenebrio.

Another main group of the dendrogram consists of various non-SNMP members of the CD36 protein family. In this tree they are serving as outgroup. Various CD36 
Results

family members of D. melanogaster, but not DmelSNMP, are clustered in this branch. For example DmelNinaD, a protein that enables cells to uptake carotenoid and DmelSantaMaria, a protein, that is involved in defense response (Hauling et al., 2014; Voolstra et al., 2006). Additionally, mammalian scavenger receptors are part of this branch, e.g. the Human CLA-1, a receptor for high density lipoprotein (HDL) and apoptotic thymocytes (Murao et al., 1997). 
Results

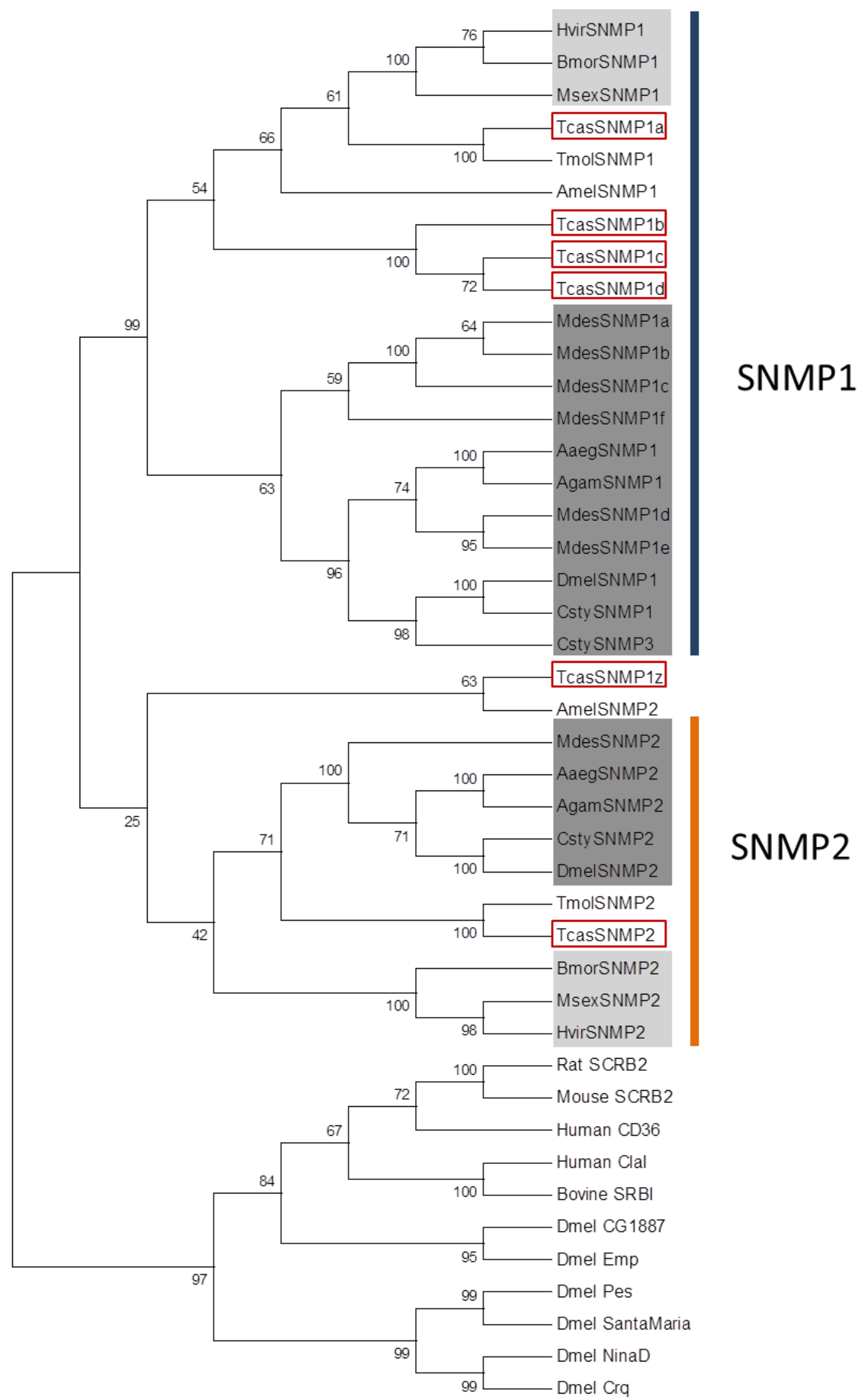

Figure 4.4 Phylogenetic analysis of SNMPs and other CD36 proteins

A bootstrap consensus tree was calculated using the neighbor joining algorithm (Saitou and Nei, 1987). Numbers next to the nodes indicate the percentage of replicate trees in which the proteins clustered together in the bootstrap test (1000 replicates). The positions of the T. castaneum SNMP 
Results

proteins are highlighted with a red frame. Dipteran SNMP proteins are shown with a dark grey background. Lepidopteran SNMP proteins are highlighted with a light grey background. Abbreviations: CD36 and LIMPII analogous-1 (CLA-1), Scavenger receptor class $B$ member 1 (SRB1), Scavenger receptor class $B$ member 2 (SCRB2), D. melanogaster scavenger receptor acting in neural tissue and majority of rhodopsin is absent (DmelSantaMaria), D. melanogaster epithelial membrane protein (DmelEmp), D. melanogaster neither inactivation nor afterpotential D (DmelNinaD), D.melanogaster croquemort (DmelCrq), D. melanogaster debris buster (DmelCG1887), D. melanogaster (Dmel), Apis mellifera (Amel), Aedes aegypti (Aaeg), Anopheles gambiae (Agam) Heliothis virescens (Hvir), the tobacco hornworm Manduca sexta (Msex), Bombyx mori (Bmor), Tenebrio molitor (Tmol) Mayetiola destructor (Mdes), Calliphora stygia (Csty)

\subsection{Localization of SNMP expressing cells}

The isolation of full length cDNA of six different TcSNMP homologs confirmed the previous finding from transcriptome data that these are expressed in antennae of T. castaneum. However, the identity of SNMP expressing cells within the antennae is diverse among insect species. In moths, SNMP1 is expressed exclusively in olfactory sensory neurons (OSNs), whereas SNMP2 is expressed in adjacent supporting cells (Forstner et al., 2008). In contrast, in D. melanogaster SNMP1 is expressed in both, OSNs and supporting cells (Benton et al., 2007). To clarify the localization of six different SNMPs within the antenna of T.castaneum, fluorescence in situ hybridization (FISH) was used. This technique has been successfully applied to visualize and identify cells within antennae of many different insect species that express a gene of interest (Forstner et al., 2008; Guo et al., 2013; Schultze et al., 2012). At the beginning of this work, this method had not been applied successfully on T. castaneum antennae. Therefore, it was necessary to establish a working FISH protocol for T.castaneum.

\subsubsection{Establishment of a FISH protocol for T. castaneum antennae}

Two different cell types have a described role for the olfactory system in insects: OSNs and associated supporting cells (Keil, 1999). The ability to visualize transcripts within both kinds of cells was a requirement for the localization and reliable identification (in double FISH, see below) of SNMP expressing cells. For 
that purpose, antisense probes targeting transcripts of highly expressed genes putatively found in OSNs and supporting cells were synthesized. As negative control also sense probes against these genes were synthesized. For OSNs, an antisense probe targeting the mRNA of the odorant receptor coreceptor (Orco) was prepared. This highly-conserved coreceptor is expressed in the majority of OSNs in different insect species (Stengl and Funk, 2013; Vosshall et al., 1999). In order to visualize supporting cells, a probe against the odorant binding protein $9 b(O B P 9 b)$ transcript was prepared. Odorant binding proteins (OBPs) are expressed in secretory supporting cells (Steinbrecht et al., 1992) and TcOBP9b as well as TcOBBP9a are some of the highest expressed OBPs in T. castaneum antennae (Dippel et al., 2014).

Due to the impermeability and hardness of the coleopteran cuticle, it was not possible to perform a whole mount FISH or FISH on thin sections as described for other insects (Krieger et al., 2004; Schymura et al., 2010). To enable penetration of the hybridization components, the antennae were longitudinally bisected (chapter 3.4.3). By using HNPP/Fast Red staining (chapter 3.4.3), it was possible to visualize transcripts of $\operatorname{Orco}$ (figure $4.5 \mathrm{~A}$ ) and $O B P 9 b$ (figure $4.5 \mathrm{~B}$ ) each.

In both images, the projected z-stack of a representative last antennal segment (segment eleven) is shown. The autofluorescence of the cuticle helps to visualize the structure of the segment. In both images distinct red signals are found within the segment. The cells labelled by the red signals of the Orco probe appear round in shape compared to the cells labeled by the $O B P 9 \mathrm{~b}$ probe which have a more elongated shape. These cell shapes correspond well to the previously described cell morphologies of Orco expressing OSNs and $O B P$ expressing secretory supporting cells (Forstner et al., 2006; Krieger et al., 2005; Schymura et al., 2010). In both FISH stainings the inner cell structure contains a darker area where the FISH staining appears ommitted. Nuclear stainings with DAPI revealed that this area represents the cell nucleus (figure 4.6). The nucleus does not contain transcripts in the same abundance as the cytoplasm and is therefore not visualized by the transcript targeting probe. Both, the typical cell morphology visible from the staining signal and the cell nucleus that lacks a visible signal confirm that specific cells of the antennal segment were labelled by FISH. Conclusively, it is possible to 
label antennal olfactory cells of T. castaneum using the established protocol of this study.

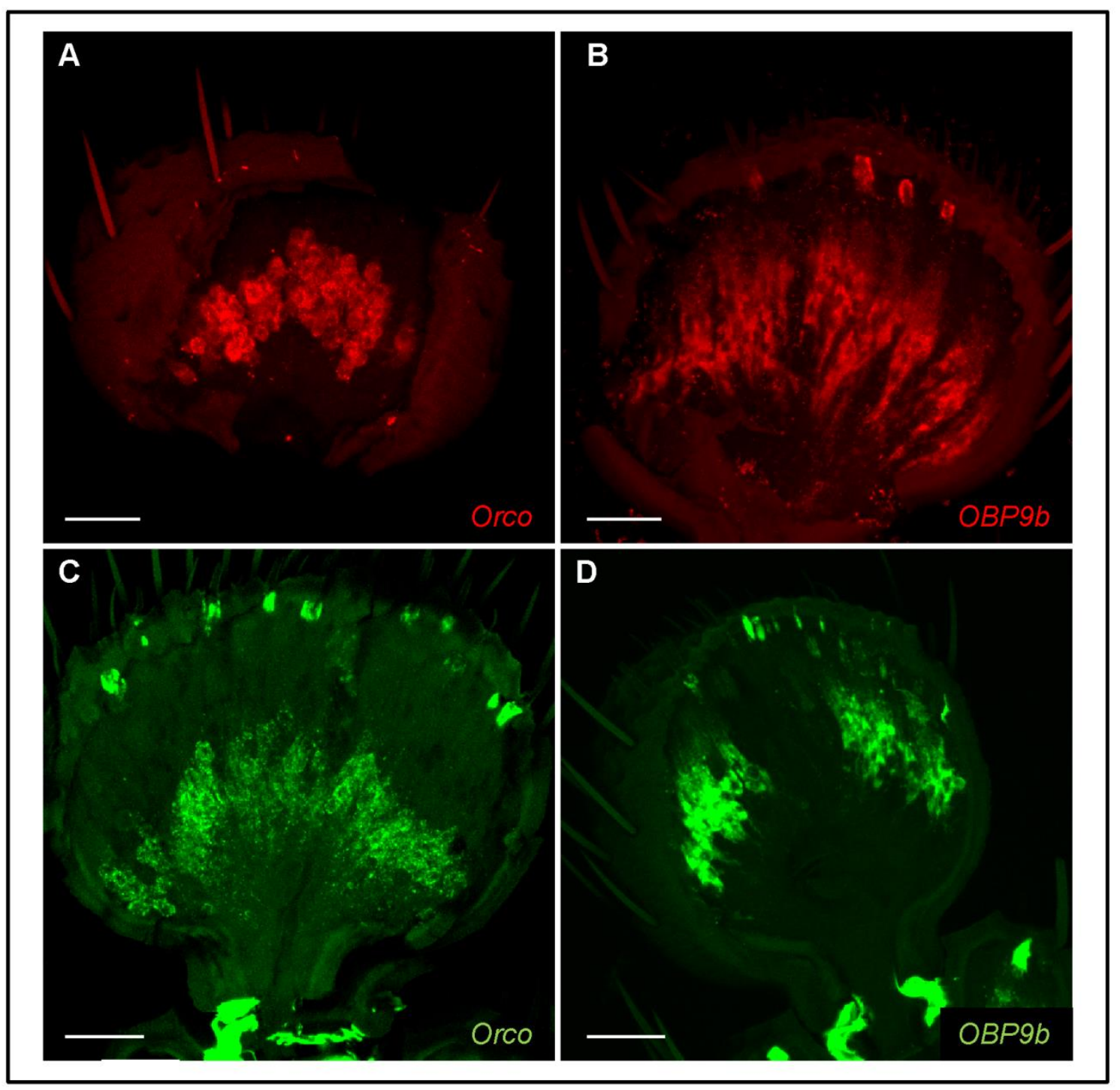

Figure 4.5 FISH visualizing marker transcripts of OSNs (Orco) and secretory supporting cells $(O B P 9 b)$ in the most distal $T$. castaneum antennal segment

A: Orco expression in antennal segment 11 of $T$. castaneum. Orco transcripts were detected using a digoxigenin-labelled antisense RNA probe and the HNPP/FastRed detection system, resulting in red fluorescence. B: $O B P 9 b$ expression in antennal segment 11 of $T$. castaneum. $O B P 9 b$ transcripts were detected using a digoxigenin-labelled RNA antisense probe and the HNPP/FastRed detection system, resulting in red fluorescence. C: Orco expression in antennal segment 11 of $T$. castaneum. Orco transcripts were detected using a biotin-labelled antisense RNA probe and the TSA detection system, resulting in green fluorescence D: $O B P 9 \mathrm{~b}$ expression in antennal segment 11 of $T$. castaneum. $O B P 9 b$ transcripts were detected using a biotin-labelled RNA antisense probe and the TSA detection system, resulting in green fluorescence.

Depicted are projected image stacks. Scale bar is $20 \mu \mathrm{m}$. 


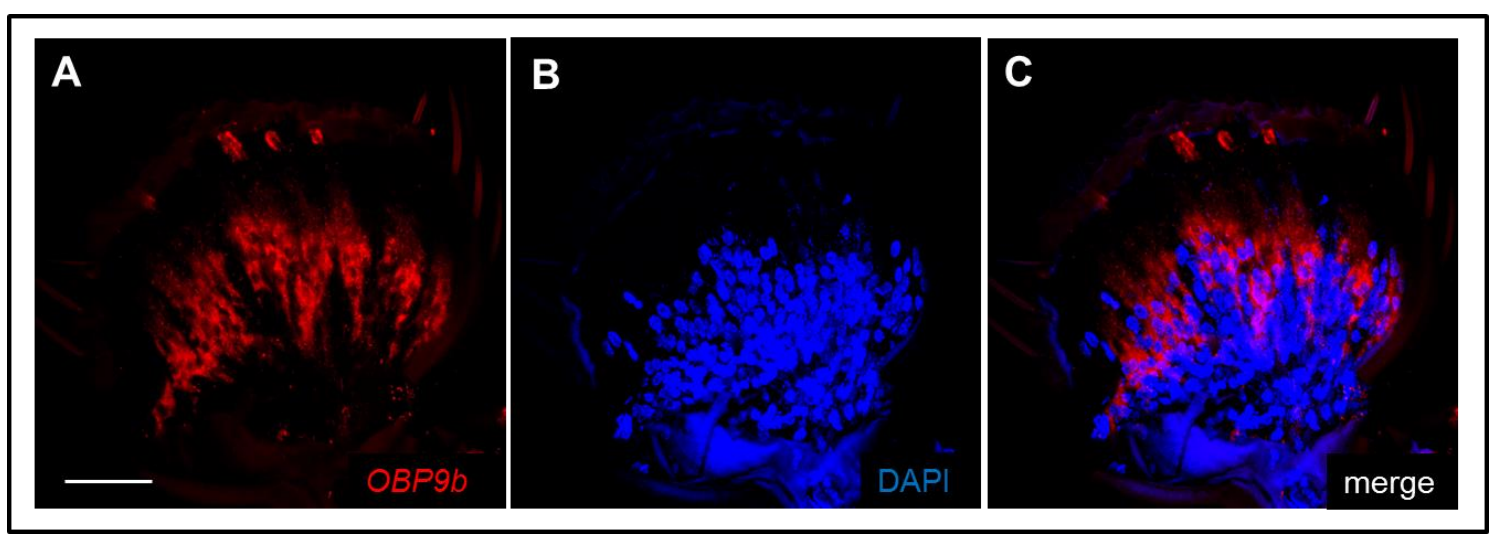

Figure 4.6 FISH and nuclear DAPI staining on T. castaneum antenna

Shown are projected images of the antennal segment 11 after FISH with a digoxigenin-labelled probe targeting $O B P 9 b$ transcripts and a nuclear DAPI staining (blue fluorescence B). Transcripts were visualized by the HNPP/FastRed detection system (red fluorescence, A). The separate fluorescence channels (A and B) and the overlay of both fluorescence channels are shown (C). Scale bar is $20 \mu \mathrm{m}$.

\subsubsection{Establishment of a double FISH protocol for T. castaneum antennae}

To reliably identify and visualize the expression of more than one type of gene transcript in different cells, it is a precondition to make use of alternative labeling methods. To test an alternative staining approach using green fluorescence, biotinlabelled probes against Orco and $O B P 9 b$ were synthesized and the TSA (tyramide signal amplification) detection system was applied (chapter 3.4.3). Cells containing Orco or $O B P 9 b$ transcripts were thereby visualized by green fluorescence and were distinctly detectable (figure 4.5 C, D). The Orco expressing cells show a round morphology, whereas the $O B P 9 b$ expressing supporting cells have an elongated cell body. The dark area of the cell nucleus is visible. In summary, the detection and visualization of transcripts within OSNs and supporting cells using biotinlabelled probes and the TSA detection system confirmed the results obtained by the HNPP/FastRed detection system.

By using both described methods and alternative staining procedures successively, it was possible to label two different transcripts with two different fluorescent dyes at once in one T. castaneum antenna (figure 4.7). All antennal club segments that are responsible for the sense of smell are shown. The Orco transcripts expressed by OSNs and $O B P 9 b$ transcripts expressed by secretory supporting cells were detected by digoxigenin- and biotin-labelled probes, respectively, and the 
hybridized probes were subsequently visualized by red or green fluorescence within the respective cells of all shown segments (figure 4.7). The red and the green staining are localized exclusively and therefore clearly distinguishable. Nonspecific fluorescence is detectable between single segments and at the segments distal cuticle where the olfactory sensilla project outwards (figure 4.7, white arrows). The performed double FISH also confirmed the previosly described cellular organization within one olfactory segment (Roth and Willis, 1951): OSNs and supporting cells are located distant from the sensillar hairs that are located at the distal end of each segment (figure 4.7). Furthermore, they are arranged in different layers within one segment. Whereas the cellbodies of the OSNs are in the proximal area of a segment (figure 4.7, red fluorescence), the supporting cells are located more distal (figure 4.7, green fluorescence). Taken together, a reliable double FISH protocol for T. castaneum antennae was established in this work.

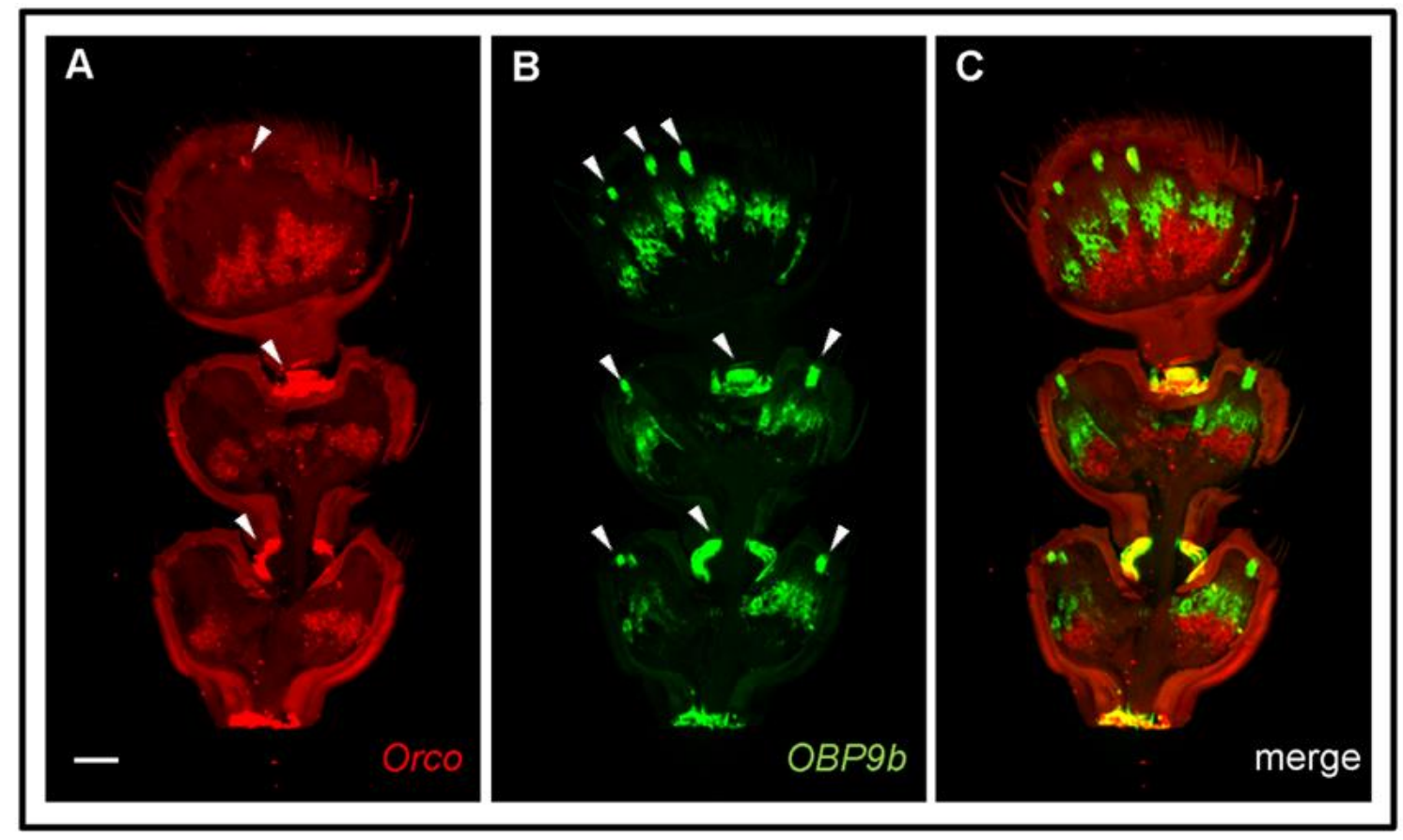

Figure 4.7 Double FISH visualizing marker transcripts of OSNs (Orco) and secretory supporting cells $(O B P 9 b)$ in $T$. castaneum club segments

Depicted are projected image stacks of an antennal stretch of segments $9-11$ of $T$. castaneum after double FISH with a digoxigenin-labelled probe targeting Orco transcripts and a biotin-labelled probe targeting $O B P 9 b$ transcripts. Hybridized transcripts were visualized by the HNPP/FastRed detection system for digoxigenin-labelled probes (red fluorescence, A) followed by the TSA detection system for biotin-labelled probes (green fluorescence, B). The separate fluorescence channels (A and B) and the overlay of both fluorescence channels are shown (C). White arrows 
point to non-specific fluorescence between single segments and at the segments distal cuticle where the olfactory sensilla rise. Scale bar is $20 \mu \mathrm{m}$.

\subsubsection{Visualization and characterization of SNMP expressing cells}

For the visualization of SNMP expressing cells the established FISH protocol was applied (chapter 4.2.1). Subsequently, the SNMP expressing cells were reliably identified by double FISH targeting the transcript of a desired SNMP and a marker transcript for OSNs or supporting cells (chapter 4.2.2). Here, visualized Orco transcripts were used as marker for OSNs, given that Orco is expressed in a majority of OSNs (figures 4.5 and 4.7). In contrast, each individual $O B P$ is expressed in a specific subset of supporting cells. Therefore double FISH with probes targeting a specific $O B P$ transcript is less effective as marker for supporting cells. However, due to the arrangement in different layers of OSNs and supporting cells (figure 4.7), a double FISH with a probe targeting the transcript of a desired SNMP with a probe targeting one of the different OBPS was used to get an impression of the localization of the SNMP expressing cell.

\subsubsection{SNMP1a is expressed in a wide range of OSNs}

To localize SNMP1a expressing cells, FISH was performed using a digoxigeninlabelled probe targeting SNMP1a transcripts on antennae of adult T. castaneum. (figure 4.8 A). All antennal club segments (segments 9 to 11) house many cells with hybridized and visualized SNMP1a transcripts. The numerous hybridization signals obtained are located in the proximal layer of the segments (figure 4.8 A). The labelled cells appear round in shape and are found exclusively within the antennal club segments. Segments 1-8 did not show any staining (data not shown). This indicates that SNMP1a is expressed in cells of the olfactory system, because the olfactory sensilla are also located exclusively on antennal club segments (Dippel et al. in preparation). To determine in which cell type SNMP1a is expressed, double FISH experiments were performed. For that purpose a digoxigenin-labelled SNMP1a probe was combined with a biotin-labelled probe 
Results

targeting Orco transcripts. In the representative eleventh antennal segment, SNMP1a expressing cells were visualized with red fluorescence (figure $4.8 \mathrm{~B}$ ) and Orco expressing cells were visualized with green fluorescence (figure $4.8 \mathrm{C}$ ). The overlay of both hybridized and visualized transcripts (figure 4.8 D) and the magnification of the orange box (figure $4.8 \mathrm{~F}, \mathrm{H}$ and $\mathrm{J}$ ) illustrates that the shown cell is visualized by both probes. All other SNMP1a expressing cells show also Orco expression.

This suggests that SNMP1a expressing cells are olfactory sensory neurons. However, there are additional Orco expressing cells that are not visualized by the SNMP1a probe. This is demonstrated by higher magnification of the blue framed area (figure 4.8 E, G and I). At the position of the green fluorescent signal of the Orco probe (figure $4.8 \mathrm{G}$ ) no red fluorescence is detectable (figure $4.8 \mathrm{E}$ ). In summary the results showed that SNMP1a is expressed in a majority of olfactory sensory neurons. 


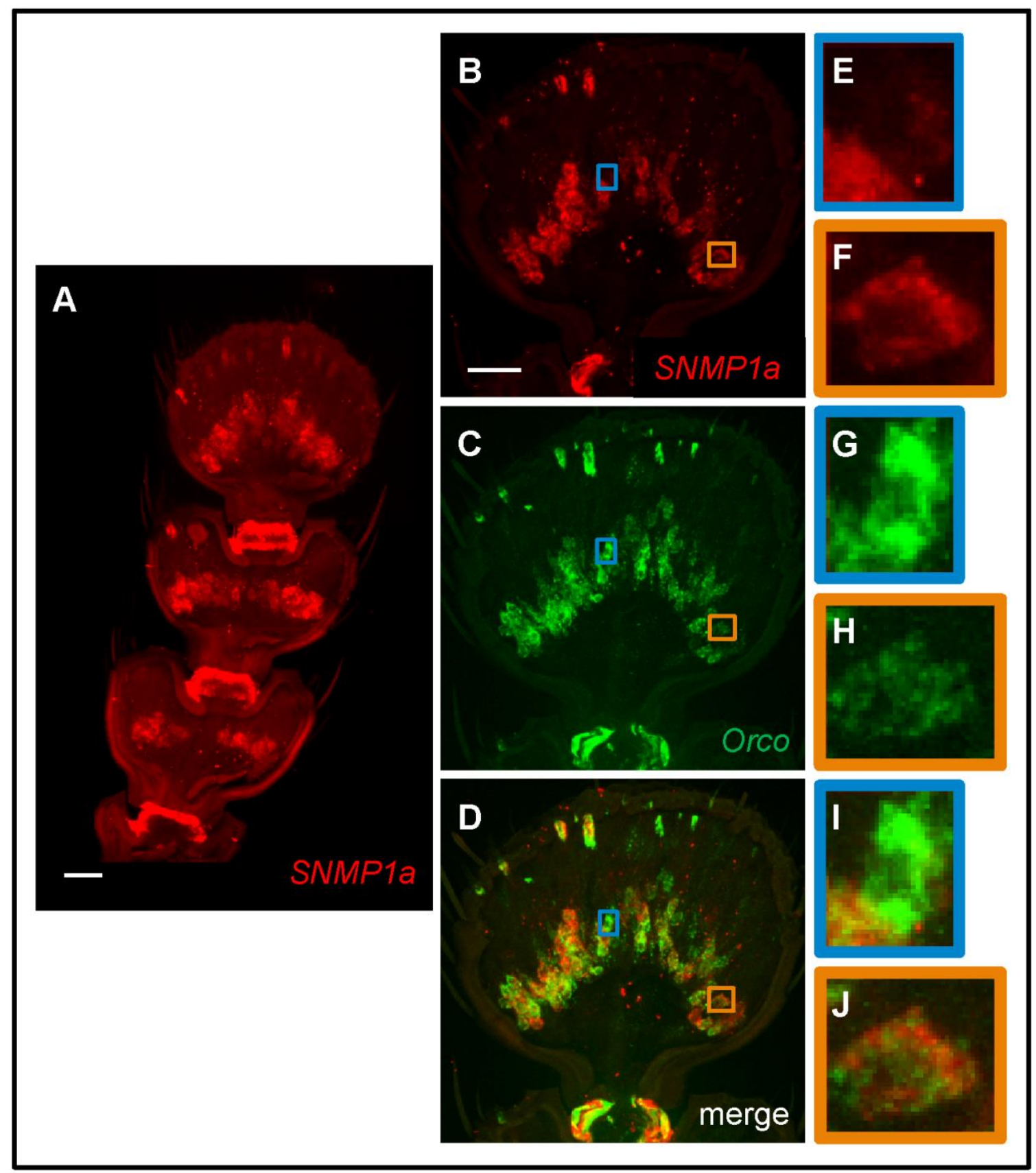

Figure 4.8 Expression pattern of SNMP1a

A: SNMP1a expression in antennal club segments of T. castaneum. SNMP1a transcripts were detected using a digoxigenin-labelled antisense RNA probe and the HNPP/FastRed detection system, resulting in red fluorescence. Confocal image stacks were projected. Scale bar is $20 \mu \mathrm{m}$.

B-D: Projected images of the antennal segment 11 after double FISH with a digoxigenin-labelled probe targeting SNMP1a transcripts and a biotin-labelled probe targeting Orco transcripts. Transcripts were visualized by the HNPP/FastRed detection system for digoxigenin-labelled probes (red fluorescence, B) followed by the TSA detection system for biotin-labelled probes (green fluorescence, C). The separate fluorescence channels (B and C) and the overlay of both fluorescence channels are shown (D). Scale bar is $20 \mu \mathrm{m}$.

E-J: Higher magnification of the boxed areas of B-D. Different areas are marked with an orange or blue box, respectively. The orange box illustrates a cell that was visualized by a probe targeting 
Results

Orco transcripts and by a probe targeting SNMP1a transcripts The blue box shows a cell that was visualized by a probe targeting Orco transcripts but not by a probe targeting SNMP1a transcripts.

\subsubsection{SNMP1b is expressed in a small number of supporting cells}

To localize SNMP1b expressing cells, FISH was carried out. Due to the high sequence similarity of the two isoforms SNMP1b.1 and SNMP1b.2, these isoforms were investigated together with the same biotin-labelled RNA probe. A representative antennal club (segments 9 -11) subjected to in situ hybridization with this probe showed that visualized cells are located in the distal region of the eleventh segment (figure 4.9 A). In the other segments no signal was detectable. The extended shape of the cells with a green signal in combination with the distal localization suggests that the hybridized cells are supporting cells. To clarify this, double FISH was performed with probes against SNMP1b and OrCo as OSN marker. Depicted is an eleventh segment of a T. castaneum antenna (figure 4.9 B-D). SNMP1b expressing cells are visualized with green fluorescence (figure $4.9 \mathrm{~B}$ ), Orco expressing cells with red fluorescence (figure $4.9 \mathrm{C}$ ). The Orco expressing cells are located in a more proximal layer of the antennal segment than the $S N M P 1 b$ expressing cells. This indicates that $S N M P 1 b$ is not expressed in OSNs. This finding, in combination with the localization and shape of the cells showing the SNMP1b signal suggests that $S N M P 1 b$ is expressed in supporting cells.

To test this suggestion double FISH targeting transcripts of SNMP1b as well as $O B P 9 a$ was conducted (figure $4.9 \mathrm{E}-\mathrm{G}$ ). In the shown representative segment the green visualized $S N M P 1 b$ expressing cells (figure $4.9 \mathrm{E}$ ) are located in the same layer as the $O B P 9 a$ expressing cells (figure $4.9 \mathrm{~F}$ ). Taken together, the observations that SNMP1b expressing cells do not show expression of the neuronal marker Orco, that they are located in the same tissue layer as $O B P 9 a$ expressing supporting cells and finally that they have an elongated cell shape, indicate that $S N M P 1 b$ is expressed in a subset of supporting cells. 


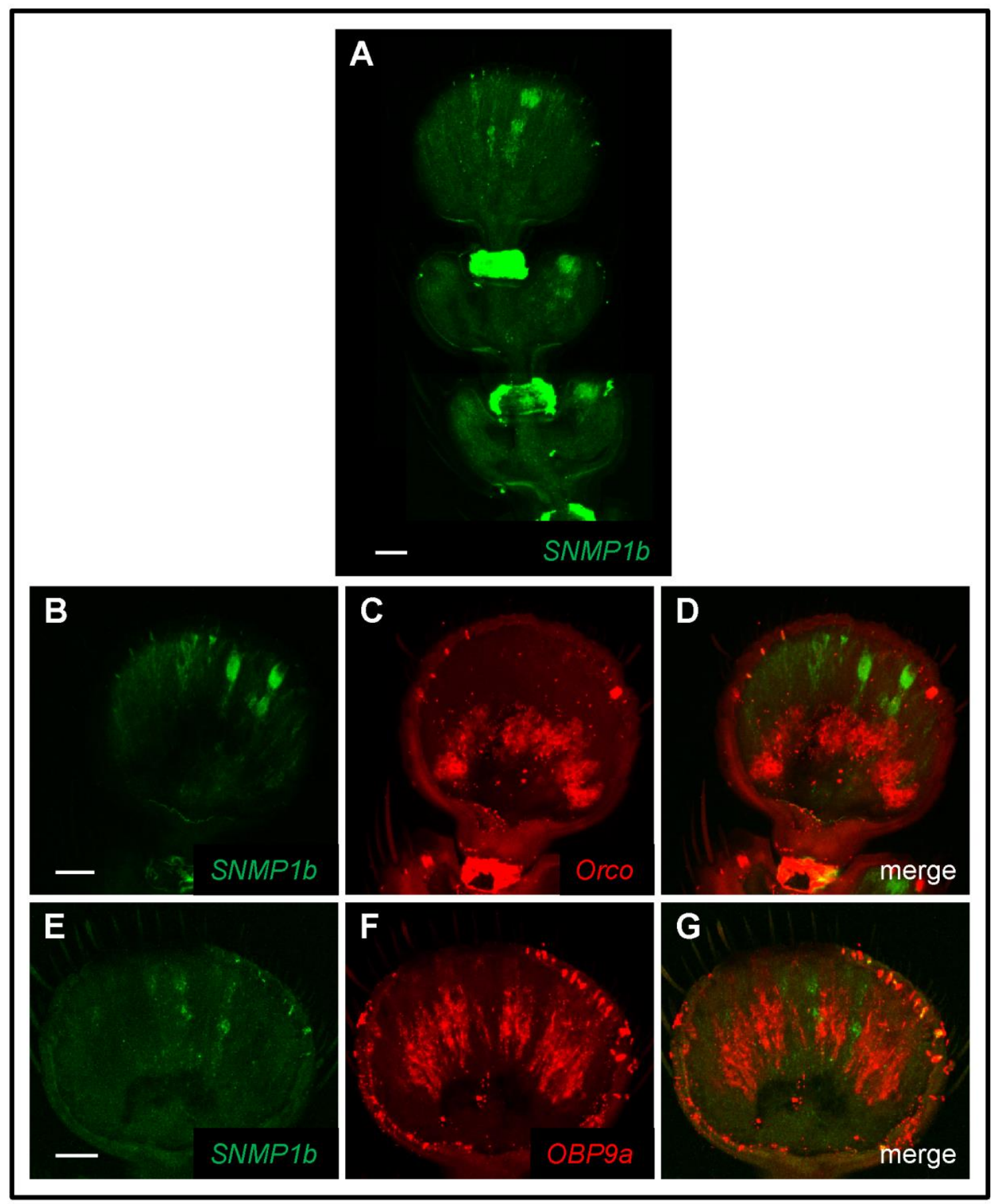

Figure 4.9 Expression pattern of SNMP1b

A: SNMP1b expression in antennal club segments (segments 9-11) of T. castaneum. SNMP1b transcripts were detected using a biotin-labelled antisense RNA probe and the TSA detection system, resulting in green fluorescence. Confocal image stacks were projected. Scale bar is $20 \mu \mathrm{m}$.

B-G: Shown are projected images of segment 11 of a T. castaneum antenna after double FISH using a biotin-labelled probe targeting $S N M P 1 b$ transcripts and a digoxigenin-labelled probe targeting either Orco (B-D) or OBP9a (E-G) transcripts. Hybridized probes were visualized by the HNPP/FastRed detection system for digoxigenin-labelled probes (red fluorescence, $\mathrm{C}$ and $\mathrm{F}$ ) followed by the TSA detection system for biotin-labelled probes (green fluorescence, $\mathrm{B}$ and $\mathrm{E}$ ). The separate fluorescence channels (B, C, E and F) and the overlay of both fluorescence channels are shown (D and G). Scale bar 20 is $\mu \mathrm{m}$. 
Results

\subsubsection{SNMP1c is expressed in numerous supporting cells}

SNMP1c expressing cells were detected using a digoxigenin-labelled probe targeting SNMP1C transcripts. In a representative eleventh segment of a T. castaneum antenna that was subjected to the decsribed FISH, red fluorescent, numerous extended shaped cells are visible in the distal part of the segment (figure $4.10 \mathrm{~A}$ ). In the other antennal club segments (segment 9 and 10) comparable hybridization signals were obtained (data not shown). To determine the identity of the SNMP1c expressing cells, double FISH was performed following the approach described for SNMP1a. A biotin-labelled probe targeting SNMP1c transcripts was combined with a digoxigenin-labelled probe targeting the OSN marker Orco. The localization and amount of the green fluorescent SNMP1C expressing cells (figure 4.10 B) is similar to the obtained hybridization signal using the digoxigenin-labelled SNMP1c probe (figure $4.10 \mathrm{~A}$ ). Compared to the Orco expressing cells, visualized by red fluorescence (figure $4.10 \mathrm{C}, \mathrm{D}$ ), the $S N M P 1 c$ expressing cells are in a more distal tissue layer (figure 4.10 D). This localization as well as the extended cell morphology of the SNMP1c expressing cells indicates that SNMP1c is expressed in supporting cells. However, when compared to the hybridization signals obtained using different $O B P$ probes (figures $4.9 \mathrm{~F}$ and $4.7 \mathrm{~B}$ ) SNMP1c expressing cells seem to be more rarely (figure 4.10). Taken together, the expression data indicate that $S N M P 1 c$ is expressed in a subclass of supporting cells that occurs numerous in the antennal club segments. 


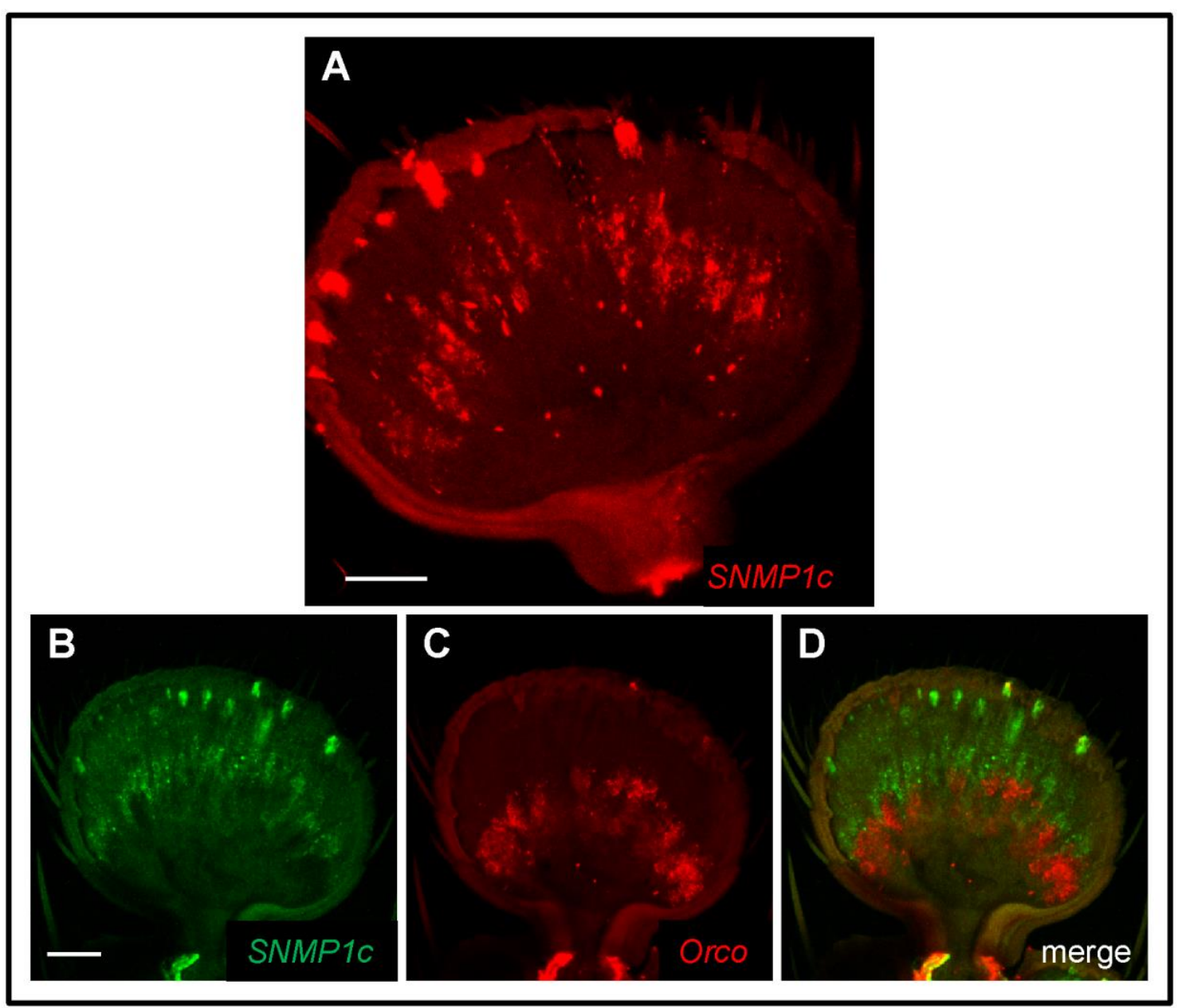

Figure 4.10 Expression pattern of SNMP1C

A: SNMP1c expression in antennal segment 11 of T. castaneum. SNMP1c transcripts were detected using a digoxigenin-labelled antisense RNA probe and the HNPP/FastRed detection system, resulting in red fluorescence. Confocal image stacks were projected. Scale bar is $20 \mu \mathrm{m}$.

B-D: Shown are projected images of segment 11 of an antenna of T. castaneum after double FISH using a biotin-labelled probe targeting SNMP1c transcripts and a digoxigenin-labelled probe targeting Orco transcripts. Hybridized probes were visualized by the HNPP/FastRed detection system for digoxigenin-labelled probes (red fluorescence, C) followed by the TSA detection system for biotin-labelled probes (green fluorescence, B). The separate fluorescence channels (B and C) and the overlay of both fluorescence channels are shown (D). Scale bar is $20 \mu \mathrm{m}$.

\subsubsection{SNMP1d is expressed in a small number of OSNs}

SNMP1d expressing cells in T. castaneum antennae were detected using FISH. A representative antennal club (segments 9 - 11) subjected to in situ hybridization with a digoxigenin-labelled probe targeting SNMP1d transcripts shows that the last segment (segment 11) contains several single visualized cells (figure $4.11 \mathrm{~A}$ ). In the case of the shown antenna, seven round shaped cells are visible. In general, 
Results

depending on the individual cutting plane of a given cryotome bisection, up to ten SNMP1d expressing cells were detectable. The SNMP1d expressing cells are exclusively located in the last club segment (segment 11; figure $4.11 \mathrm{~A}$ ). The round morphology of the visualized cells indicated that SNMP1d is expressed in OSNs. To confirm this suggestion, FISH using probes targeting SNMP1d transcripts (digoxigenin-labelled) and Orco transcripts (biotin-labelled) was conducted.

In a representative eleventh segment, subjected to the described double FISH, SNMP1d expressing cells visualized with red fluorescence and Orco expressing cells visualized with green fluorescence are detectable (figure $4.11 \mathrm{~B}-\mathrm{D}$ ). The white framed area shows a cell that contains Orco transcripts as well as SNMP1d transcripts (figure 4.11 E-G). This shows that this cell expresses both genes, SNMP1d and Orco. In summary, the results suggest, that SNMP1d is expressed in a small number of OSNs that are exclusively located in the last segment. 


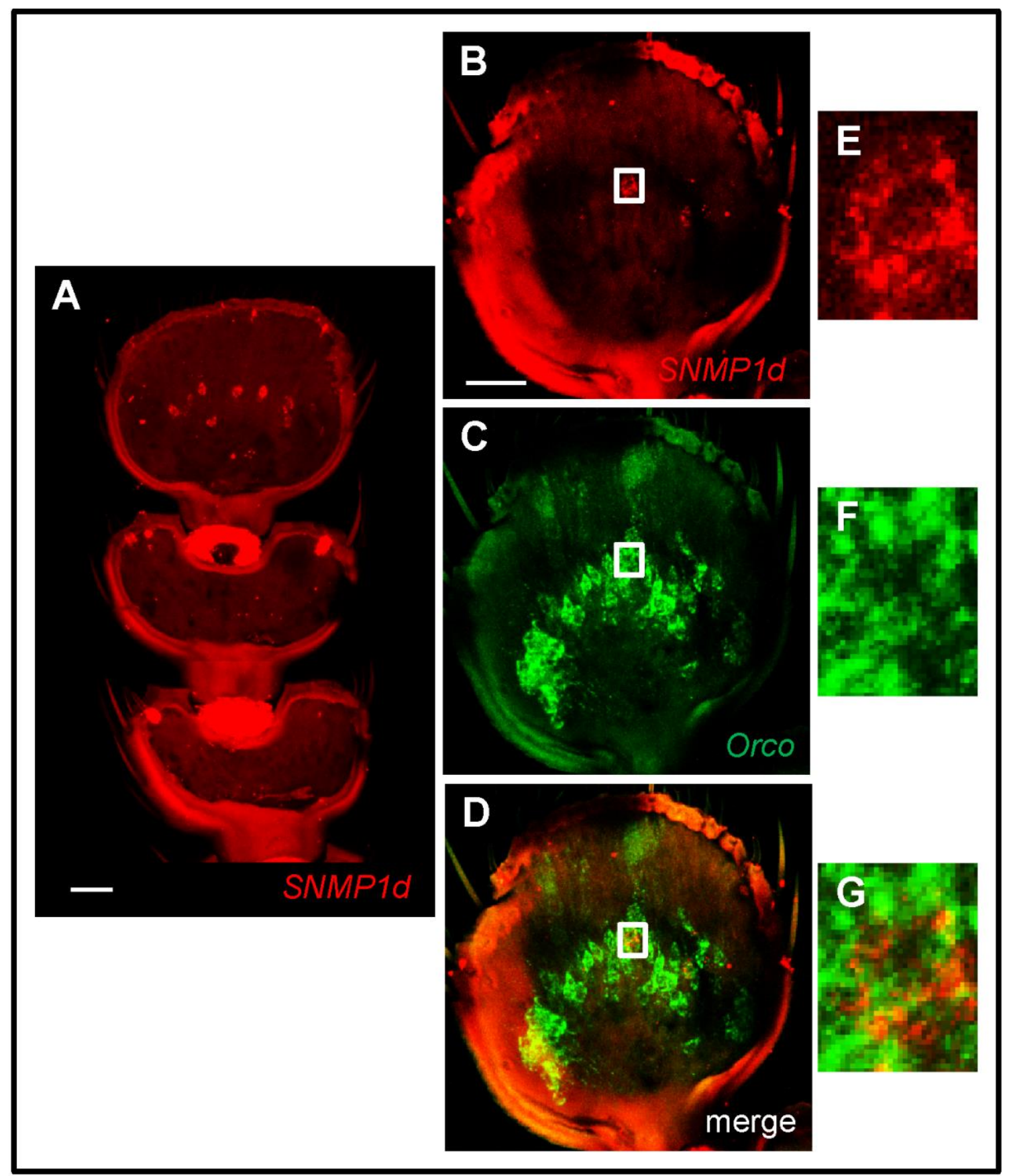

Figure 4.11 Expression pattern of SNMP1d

A: SNMP1d expression in antennal club segments (segments 9-11) of T. castaneum. SNMP1d transcripts were detected using a digoxigenin-labelled antisense RNA probe and the HNPP/FastRed detection system, resulting in red fluorescence. Confocal image stacks were projected. Scale bar is $20 \mu \mathrm{m}$.

B-D: Shown are projected images of the antennal segment 11 after double FISH with a digoxigeninlabelled probe targeting SNMP1d transcripts and a biotin-labelled probe targeting Orco transcripts. Transcripts were visualized by the HNPP/FastRed detection system for digoxigenin-labelled probes (red fluorescence, B) followed by the TSA detection system for biotin-labelled probes (green fluorescence, $C$ ). The separate fluorescence channels (B and C) and the overlay of both fluorescence channels are shown (D). Scale bar is $20 \mu \mathrm{m}$.

E-G: Higher magnification of the white framed areas of B-D. Shown is a cell that was visualized by a probe targeting Orco transcripts and by a probe targeting SNMP1d transcripts 
Results

\subsubsection{SNMP1z is expressed in single supporting cells}

To visualize SNMP1z expressing cells, FISH with a digoxigenin-labelled probe targeting SNMP1z transcripts was applied. Within the illustrated T. castaneum club segments (segment 9-11), a single cell located in segment eleven shows SNMP1z expression (figure $4.12 \mathrm{~A}$ ). In general, in one bisected half of an antenna a maximum of two cells showed a hybridization signal using an SNMP1z probe. These cells were always located in the last segment 11. To test, whether SNMP1z is expressed in OSNs or supporting cells, double stainings with a biotin-labelled probe either targeting Orco transcripts or targeting $O B P 9 b$ transcripts were performed. The SNMP1z expressing cell (red fluorescence, figure $4.12 \mathrm{~B}$ ) is in a different location than the Orco expressing OSNs (green fluorescence, figure 4.12 C). More precisely, the SNMP1z expressing cell is located more distal compared to the OSNs (figure $4.12 \mathrm{D}$ ), indicating that this $S N M P 1 z$ expressing cell is a supporting cell. The double FISH using probes targeting SNMP1z transcripts and $O B P 9 b$ transcripts (figure $4.12 \mathrm{E}-\mathrm{G}$ ) showed that the $S N M P 1 z$ expressing cell (figure $4.12 \mathrm{E}$ ) is located in the same tissue layer like the $O B P 9 b$ expressing supporting cells (figure $4.12 \mathrm{~F}$ ). In summary, the results that $S N M P 1 z$ is not expressed in Orco expressing OSNs but in the tissue layer of $O B P 9 b$ expressing supporting cells suggests that SNMP1z is expressed in single supporting cells. 


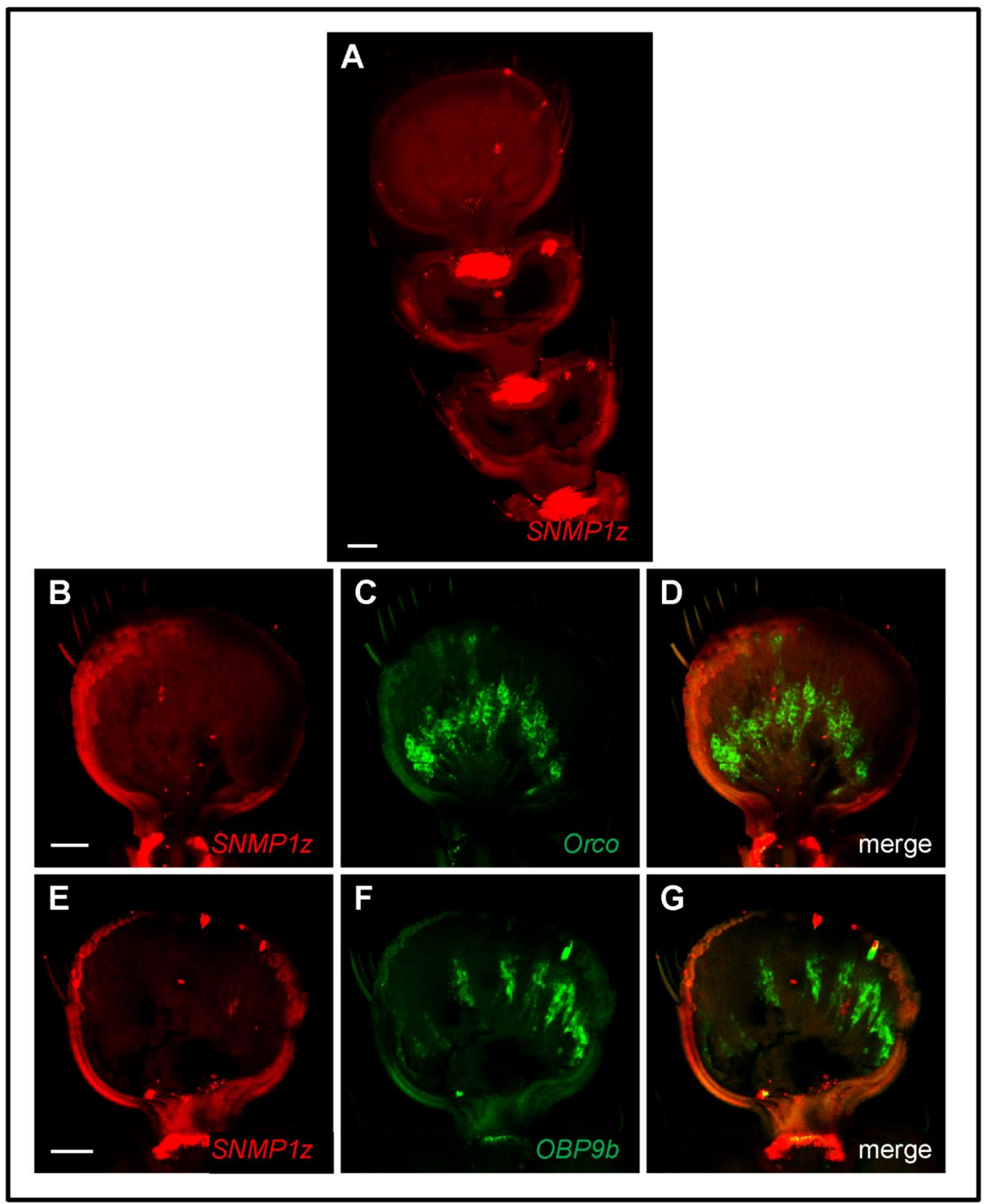

Figure 4.12 Expression pattern of SNMP1z

A: $S N M P 1 z$ expression in antennal club segments (segments 9-11) of T. castaneum. SNMP1z transcripts were detected using a digoxigenin-labelled antisense RNA probe and the HNPP/FastRed detection system, resulting in red fluorescence. Confocal image stacks were projected. Scale bar is $20 \mu \mathrm{m}$.

B-G: Shown are projected images of segment 11 of a T. castaneum antenna after double FISH using a digoxigenin-labelled probe targeting $S N M P 1 z$ transcripts and a biotin-labelled probe targeting either Orco (B-D) or OBP9b (E-G) transcripts. Hybridized probes were visualized by the HNPP/FastRed detection system for digoxigenin-labelled probes (red fluorescence, B and E) followed by the TSA detection system for biotin-labelled probes (green fluorescence, $\mathrm{C}$ and F). The separate fluorescence channels (B, C, E and F) and the overlay of both fluorescence channels are shown (D and G). Scale bar is $20 \mu \mathrm{m}$. 
Results

\subsubsection{FISH did not reveal SNMP2 expressing cells in the antennal club segments}

To visualize SNMP2 expressing cells of T. castaneum antenna, FISH was conducted. Digoxigenin-labelled probes as well as biotin-labelled probes against transcripts of SNMP2 were used and different hybridization conditions were tested. It was not possible to visualize transcripts of SNMP2 in antennal tissue under all tested conditions (data not shown).

\subsubsection{Cellular organization of SNMP expressing cells within one segment}

As shown previously, the number and distribution of SNMP expressing cells is diverse within the antennal club segments of T. castaneum (chapter 4.2.3). While SNMP1a and SNMP1c expression was found in many cells, SNMP1b, SNMP1d, and SNMP1z were found to be expressed in single cells. It was further shown that the $S N M P$ expressing cell types are different: $S N M P 1 b, S N M P 1 c$ and $S N M P 1 z$ are very likely expressed in supporting cells whereas both SNMP1a and SNMP1d are expressed in OSNs. To clarify the cellular organization of the different SNMP expressing cells within one segment, double FISH with probes targeting two different SNMPS were conducted.

\subsubsection{SNMP1a and SNMP1d are expressed in different subtypes of OSNs}

In other insects just one SNMP1 homolog has been shown to be expressed in OSNs (Benton et al., 2007; Forstner et al., 2008). However, FISH studies on antennal club segments of $T$. castaneum revealed that two different $T$. castaneum SNMP1 homologs, SNMP1a and SNMP1d, are expressed in OSNs (chapter 4.2.3).

To test whether T. castaneum has different subclasses of OSNs expressing two different SNMP1 homologs, double FISH experiments were performed using probes targeting the SNMP1a transcript (green fluorescence, figure $4.13 \mathrm{~B}$ ) and the SNMP1d transcript (red fluorescence, figure $4.13 \mathrm{~A}$ ). The obtained hybridization 
signals confirm the previous finding that a small number of OSNs express SNMP1d (figure $4.13 \mathrm{~A}$ ), while a large amount of OSNs express SNMP1a (figure $4.13 \mathrm{~B}$ ). The overlay of both fluorescence channels (figure $4.13 \mathrm{C}$ ) as well as the higher magnification of the area within the white box (figure $4.13 \mathrm{D}-\mathrm{I}$ ) shows that the expression of SNMP1d and SNMP1a is mutually exclusive. In summary, the results showed that SNMP1d and SNMP1a are expressed in different subtypes of OSN.

\subsubsection{T. castaneum SNMPs are partially expressed in adjacent cells}

In D. melanogaster and different moth species it has been shown that SNMP1 expressing OSNs are surrounded by secretory supporting cells, that also express an SNMP homolog (Benton et al., 2007; Forstner et al., 2008). Therefore it was tested, whether the SNMP expressing OSNs of T. castaneum are also adjacent to SNMP expressing secretory supporting cells.

To test for a spatially adjacent localization of SNMP1d expressing OSNs and SNMP1z expressing secretory supporting cells, FISH using probes targeting the transcripts of SNMP1z and SNMP1d was conducted. Hybridized SNMP1d transcripts were visualized with red fluorescence (figure $4.13 \mathrm{~J}$ ) and SNMP1z transcripts with green fluorescence (figure $4.13 \mathrm{~K}$ ). The overlay of both fluorescence channels (figure $4.13 \mathrm{~L}$ ) and the enlargement of the white marked area (figure $4.13 \mathrm{M}-0$ ) show that SNMP1d expressing OSNs and SNMP1z expressing supporting cells are located nearby. This fits to the described colocalization of SNMP expressing OSNs and supporting cells of D. melanogaster and different moth species (Benton et al., 2007; Forstner et al., 2008). However, the majority of SNMP1d expressing OSNs show no colocalization with SNMP1z expressing supporting cells (figure $4.13 \mathrm{~L}$ ). Taken together, SNMP1d expressing OSNs are partially located nearby SNMP1z expressing supporting cells.

To investigate whether SNMP1d expressing neurons are adjacent to $S N M P 1 b$ expressing supporting cells, FISH using probes targeting the transcripts of SNMP1d and $S N M P 1 b$ was performed. In the bisected antennae subjected to double FISH no colocalization was detectable, because these cells were never even found in the same bisection (data not shown). 
Results

In summary, double FISH using probes targeting different SNMP transcripts showed that SNMP1d expressing OSNs are not located nearby SNMP1b expressing supporting cells but that some SNMP1d expressing OSNs are spatially adjacent to SNMP1z expressing supporting cells. This suggests that SNMP1d and SNMP1z expressing cells can form a spatially concerted unit.

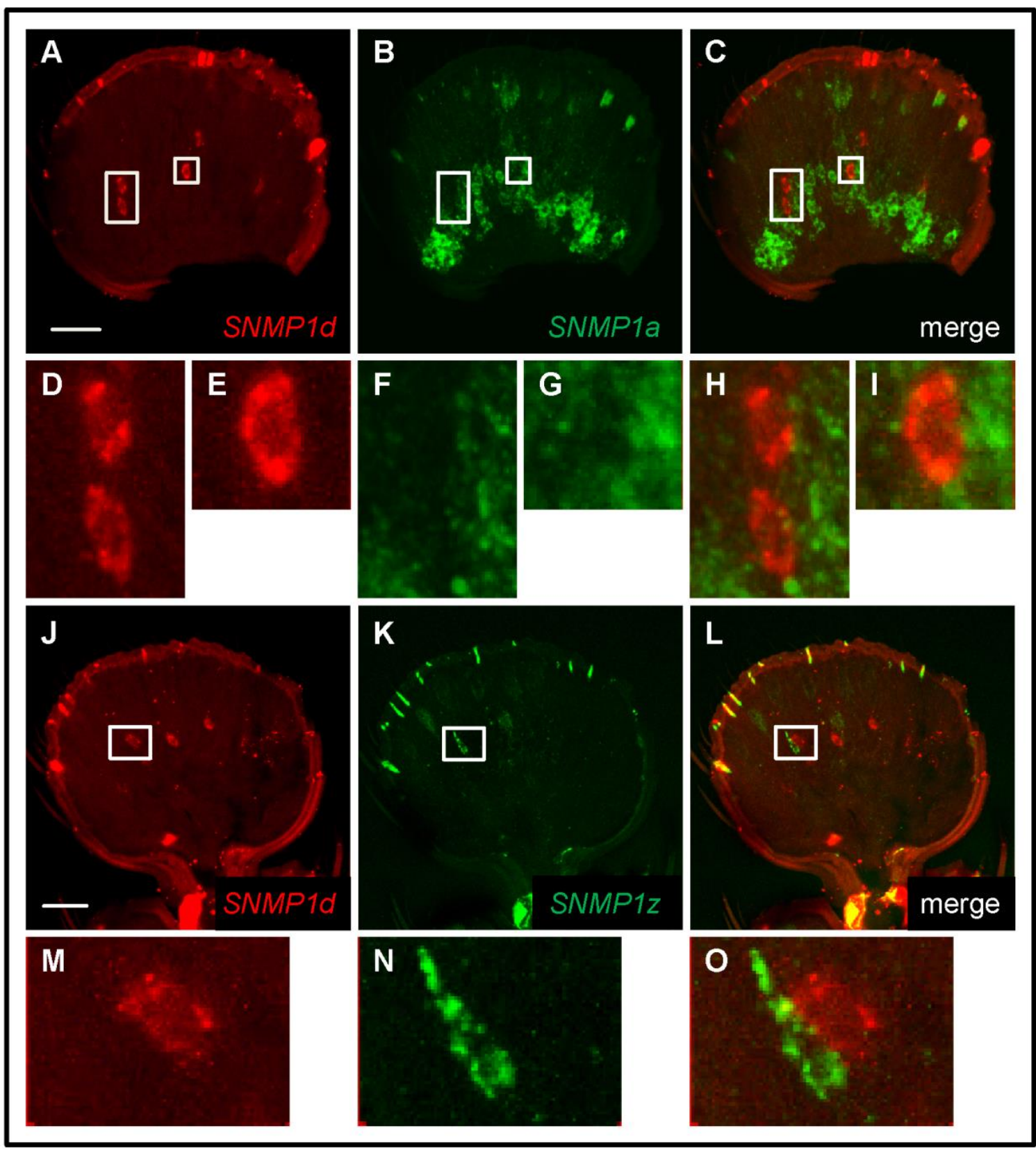

Figure 4.13 Cellular organizations of different SNMPs within one segment

A-C: Shown are projected images of the antennal segment 11 after double FISH with a digoxigeninlabelled probe targeting SNMP1d transcripts and a biotin-labelled probe targeting SNMP1a transcripts. Transcripts were visualized by the HNPP/FastRed detection system for digoxigeninlabelled probes (red fluorescence, A) followed by the TSA detection system for biotin-labelled 
probes (green fluorescence, B). The separate fluorescence channels (A and B) and the overlay of both fluorescence channels are shown (C). Scale bar is $20 \mu \mathrm{m}$. D-I: Higher magnification of the white framed areas of A-C. Shown are cells that were visualized by a probe targeting SNMP1d transcripts but not by a probe targeting SNMP1a transcripts

J-L: Shown are projected images of the antennal segment 11 after double FISH with a digoxigeninlabelled probe targeting SNMP1d transcripts and a biotin-labelled probe targeting $S N M P 1 z$ transcripts. Transcripts were visualized by the HNPP/FastRed detection system for digoxigeninlabelled probes (red fluorescence, J) followed by the TSA detection system for biotin-labelled probes (green fluorescence, K). The separate fluorescence channels (J and $\mathrm{K}$ ) and the overlay of both fluorescence channels are shown (L). Scale bar is $20 \mu \mathrm{m}$. M-O: Higher magnification of the white framed areas of J-L. Shown is the adjacent localization of two cells that were visualized by a probe targeting SNMP1d transcripts and a probe targeting SNMP1z transcripts.

\subsection{Examining the role of TcSNMPs in Tribolium castaneum}

As shown previously T. castaneum has two different TcSNMPs expressed in OSNs: TCSNMP1a is expressed in a wide range of OSNs in all three antennal club segments. In contrast, TcSNMP1d is expressed only in up to ten neurons per bisected antenna and these neurons are located exclusively in the last segment (chapter 4.3). Double FISH targeting transcripts of TcSNMP1a and TcSNMP1d revealed that these genes are expressed by a different class of OSNs (figure 4.13). Due to the described importance of neuronal SNMPs for the appropriate detection of odorants (Benton et al., 2007; Jin et al., 2008), the potential and maybe different role between these neuronal TcSNMPs was investigated in this study. Therefore each of these genes was silenced by injection of the respective dsRNA, leading to a robust systemic RNAi response in T. castaneum (Posnien et al., 2009). Additionally, control beetles were injected with dsRNA against the red fluorescent protein DsRed. DsRed is a gene from a Discosoma species and therefore has no target transcript in T. castaneum (Dietrich and Maiss, 2002). The effect of TcSNMP1a or TcSNMP1d knockdown was measured by electroantennography (EAG, conducted by Karthi Balakrishnan, Forest Zoology and Forest Conservation, University Göttingen). This method allows to measure the summation of receptor potentials of all OSNs in one antenna and gives therefore more clearly and reliably results than behavioral assays (Guo and Qing Li, 2009). After EAG measurements the knockdown of the particular transcript was confirmed by FISH. 
Results

\subsubsection{Examining the role of neuronal TcSNMPs in pheromone and beetle odor detection}

In other insects of the order Diptera and Lepidoptera, it has been shown that SNMPs are expressed in pheromone sensitive neurons and that they play a crucial role for the pheromone detection system (Benton et al., 2007; Pregitzer et al., 2014; Vogt et al., 2009). One pheromone is well described for T. castaneum, the aggregation pheromone 4-8-Dimethyldecanal (DMD) (Kim et al., 2005; Suzuki, 1980).

Furthermore, it has been shown that DmSNMP1 of D. melanogaster is expressed mainly in OSNs that are linked to the detection of unknown odors of fly extracts (Benton et al., 2007; van der Goes van Naters and Carlson, 2007). In contrast to D. melanogaster, in T. castaneum a typical beetle odor is described: 1-4benzoquinone, a substance that is produced within the stink glands (Li et al., 2013; Pappas and Morrison, 1995; Unruh et al., 1998). To test a potential involvement of TcSNMP1a or TcSNMP1d in the detection of DMD and 1-4-benzoquinone, each of these genes was silenced by RNA interference and the effect of the resulting knockdown was measured using EAG.

The control beetles (figure 4.14, grey bars) showed a dose response relation, meaning that the antennal activity increased with higher odor concentrations. Comparison of the averaged EAG responses obtained of TcSNMP1a dsRNA or TcSNMP1d dsRNA injected beetles with the control beetles revealed, that the knockdown beetles also showed a dose response relation, but with overall reduced EAG responses to all tested odor concentrations (figure 4.14). More precisely, the TCSNMP1a knockdown in females (figure 4.14, right, green bars) led to a slightly reduced detection of $\mathrm{DMD}$ as well as 1-4-benzoquinone compared to control beetles (figure 4.14, grey bars). In contrast, knockdown of TcSNMP1a in males (figure 4.14, left, green bars) had no significant effect on the detection of DMD and 1-4-benzoquinone. This is confirmed by statistical analysis (figure 4.14).

Knockdown of TCSNMP1d led to highly significant decreased responses in both sexes (figure 4.14, yellow bars). In particular, TcSNMP1d dsRNA injected beetles showed such a reduced sensitivity to DMD and 1-4-benzoquinone that high concentrated odors $\left(10^{-1}\right.$ dilution, figure 4.14$)$ resulted in a comparable response 
as responses of control beetles elicited by very diluted odors $\left(10^{-4}\right.$ dilution, figure 4.14).

In summary, knockdown of TcSNMP1a and TcSNMP1d transcripts led to decreased antennal responses towards DMD and 1-4-benzoquinone. Here, TcSNMP1a knockdown showed a stronger effect in female beetles. Generally, in both sexes TcSNMP1d knockdown led to a stronger effect than TcSNMP1a knockdown.

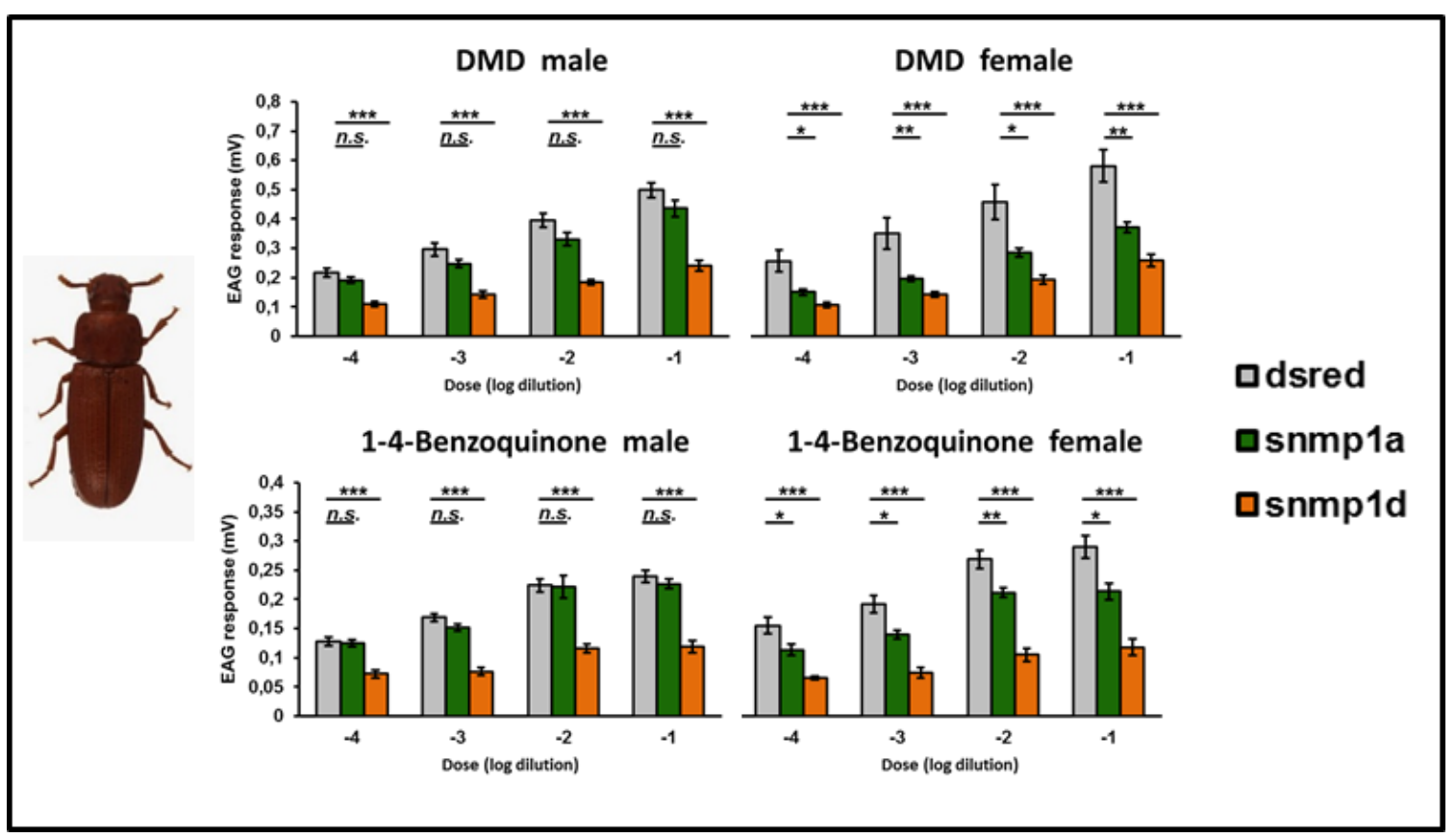

Figure 4.14 EAG responses of RNAi treated $T$. castaneum to Tribolium odors

Shown are EAG responses of indicated beetles $(n=7)$ and odor dilutions, plotted as mean values and s.e.m (standard error of the mean). EAG responses were measured by Karthi Balakrishnan (Forest Zoology and Forest Conservation, University Göttingen), after application of 4-8-dimethyldecanal (DMD) and 1-4-benzoquinone diluted in silicon oil. The asterisks indicate statistically significant differences between control beetles that were injected with dsRNA against DsRed (dsred, grey bars) and knockdown beetles injected with dsRNA against TCSNMP1a (snmp1a, green bar) or TcSNMP1d (snmp1d, yellow bar), respectively. Statistical significance was calculated using ANOVA with Bonferroni post hoc test: ${ }^{*} \mathrm{p}<0.05{ }^{* *} \mathrm{p}<0.01{ }^{* * *} \mathrm{p}<0.001$; n.s. no significant difference. Line ends indicate experimental groups showing significant differences as indicated.

\subsubsection{Examining the role of neuronal TcSNMPs for the detection of fatty acid derivatives}

To test an involvement of TcSNMP1a and TcSNMP1d in the detection of compounds that are not produced by T. castaneum but might be relevant for it, also other odors were analyzed. All known odors that require a functional SNMP for 
Results

normal response kinetics are fatty-acid derivatives (Benton et al., 2007; GomezDiaz et al., 2013; Pregitzer et al., 2014; Ronderos et al., 2014). Hence, fatty food odors and in addition pheromone like substances were tested for the requirement of TcSNMP1a and TcSNMP1d on their detection.

First, typical fatty food odors were analyzed. Phillips et al. (1993) showed that T. castaneum is attracted by wheat germ oil (WGO). It was hypothesized that older and damaged grain with a higher fatty acid content reflects the habitat preference of T. castaneum. For that purpose, antennal responses of RNAi treated beetles towards WGO and another compound of wheat, ß-ionone, were tested using EAG (Nixon, 1994).

The averaged EAG responses of seven beetles each and following statistical calculations showed that knockdown of TcSNMP1a and TcSNMP1d led to significantly decreased antennal responses in female beetles to wheat germ oil and ß-ionone for all tested odor concentrations (figure 4.15, right).

Also male beetles showed reduced EAG responses to WGO and $ß$-ionone after injection with dsRNA targeting transcripts of TcSNMP1a and TcSNMP1d (figure 4.15 left), but not as strongly reduced as in female beetles (figure 4.15, right). For both sexes and both odorants disruption of TcSNMP1d function had a stronger effect to all tested situations than knockdown of TcSNMP1a. Statistical analysis showed that this strong effect is highly significantly different when compared with the control beetles (figure 4.15).

The EAG measurements showed that SNMP1a dsRNA and SNMP1d dsRNA injected beetles of both sexes did not show a dose dependent response to different wheat germ oil concentration as obtained from the DsRed dsRNA injected control beetles (figure 4.15 upper row). These constant EAG values of the knockdown beetles are at a lower level than the response of the control group to the much diluted WGO (10-4 dilution, figure 4.15).

Taken together, knockdown of TCSNMP1a and TcSNMP1d transcripts led to decreased antennal responses towards the food odors WGO and $ß$-ionone. Here, female TcSNMP1a knockdown beetles showed a stronger effect. Generally, TcSNMP1d knockdown led to a stronger effect than TcSNMP1a knockdown in all beetles. 


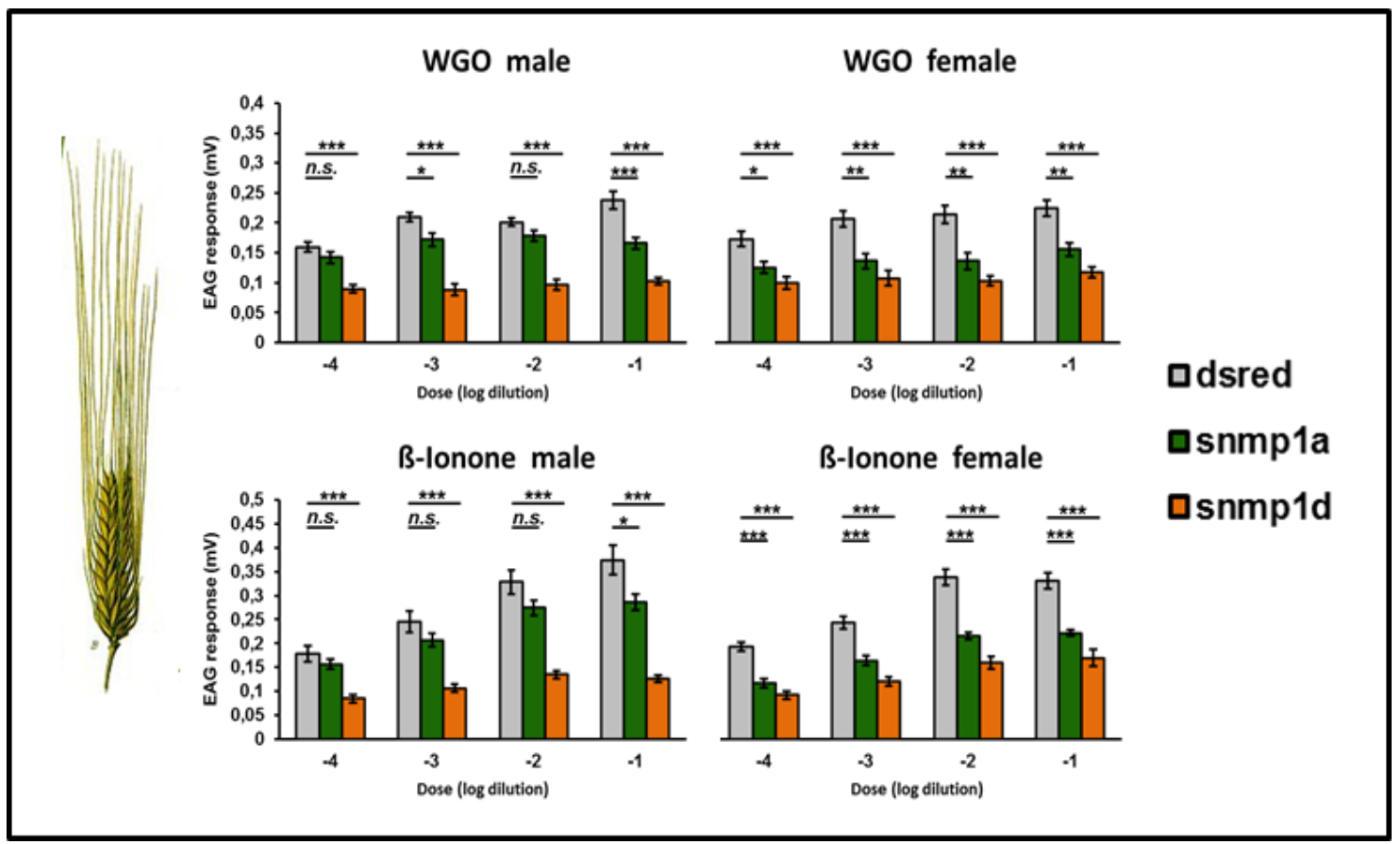

Figure 4.15 EAG responses of RNAi treated T. castaneum to fatty food odors

Shown are EAG responses of indicated beetles $(n=7)$ and odor dilutions, plotted as mean and s.e.m (standard error of the mean). EAG responses were measured by Karthi Balakrishnan (Forest Zoology and Forest Conservation, University Göttingen), after application of wheat germ oil (WGO) and $ß$-ionone diluted in silicon oil. The asterisks indicate statistically significant differences between control beetles that were injected with dsRNA against DsRed (dsred, grey bars) and knockdown beetles injected with dsRNA against TcSNMP1a (snmp1a, green bar) or TcSNMP1d (snmp1d, yellow bar), respectively. Statistical significance was calculated using ANOVA with Bonferroni post hoc test: ${ }^{*} \mathrm{p}<0.05 ;^{* *} \mathrm{p}<0.01{ }^{* * *} \mathrm{p}<0.001$; n.s. no significant difference. Line ends indicate experimental groups showing significant differences as indicated.

To test whether TcSNMP1a and TcSNMP1d are required for the detection of fatty, pheromone like plant odors, geraniol and cis-3-hexenol were analyzed. Geraniol is a component of many plant oils. It has been shown that this odor is attractive to the japanese beetle Popillia japonica (Fleming, 1969) and that it is an ingredient of the Nasonov pheromone of the honey bee Apis mellifera. This highly attractive odorant blend is used by bees to find the entrance to their colony or flowers with nectar (Schmidt, 1994; Williams et al., 1981). The fatty-acid derivative cis-3hexenol is a typical green-leaf-volatile (Abdullah and Butt, 2015).

To test for an involvement of TcSNMP1a and TcSNMP1d in the detection of geraniol and cis-3-hexenol, an analogous EAG approach as described above (chapter 4.3.1) was performed. Comparison of the obtained EAG responses of TcSNMP1a dsRNA injected male beetles (figure 4.16, left, green bars) with the EAG 
Results

responses of the control beetles (figure 4.16, left, grey bars) revealed that the measured sum potentials were in a similar range. Statistical analysis showed that there was no significant difference between TcSNMP1a dsRNA and DsRed dsRNA injected males and hereby confirming that finding. In contrast to that, TcSNMP1a dsRNA injected female beetles showed an effect (figure 4.16, right). In particular, for all tested concentrations of geraniol and cis-3-hexenol, the EAG responses were significantly decreased compared to the female control beetles.

Loss of TcSNMP1d function resulted in lower EAG responses for all tested concentrations of geraniol and cis-3-hexenol compared to control beetles (figure 4.16). This reduction was found for male and female beetles and is highly significant. In general, the effect of dsRNA injection against TcSNMP1d results in a more severe decrease of EAG responses than RNAi against TcSNMP1a.

In summary, knockdown of TCSNMP1a and TCSNMP1d led to decreased antennal responses towards the plant odors geraniol and cis-3-hexenol. Again, as for the other tested odors (chapter 4.3.1 and 4.3.2), the female beetles showed a stronger effect to theTcSNMP1a knockdown than the male beetles. Furthermore the TcSNMP1d knockdown led to a stronger effect than TcSNMP1a knockdown in both sexes.

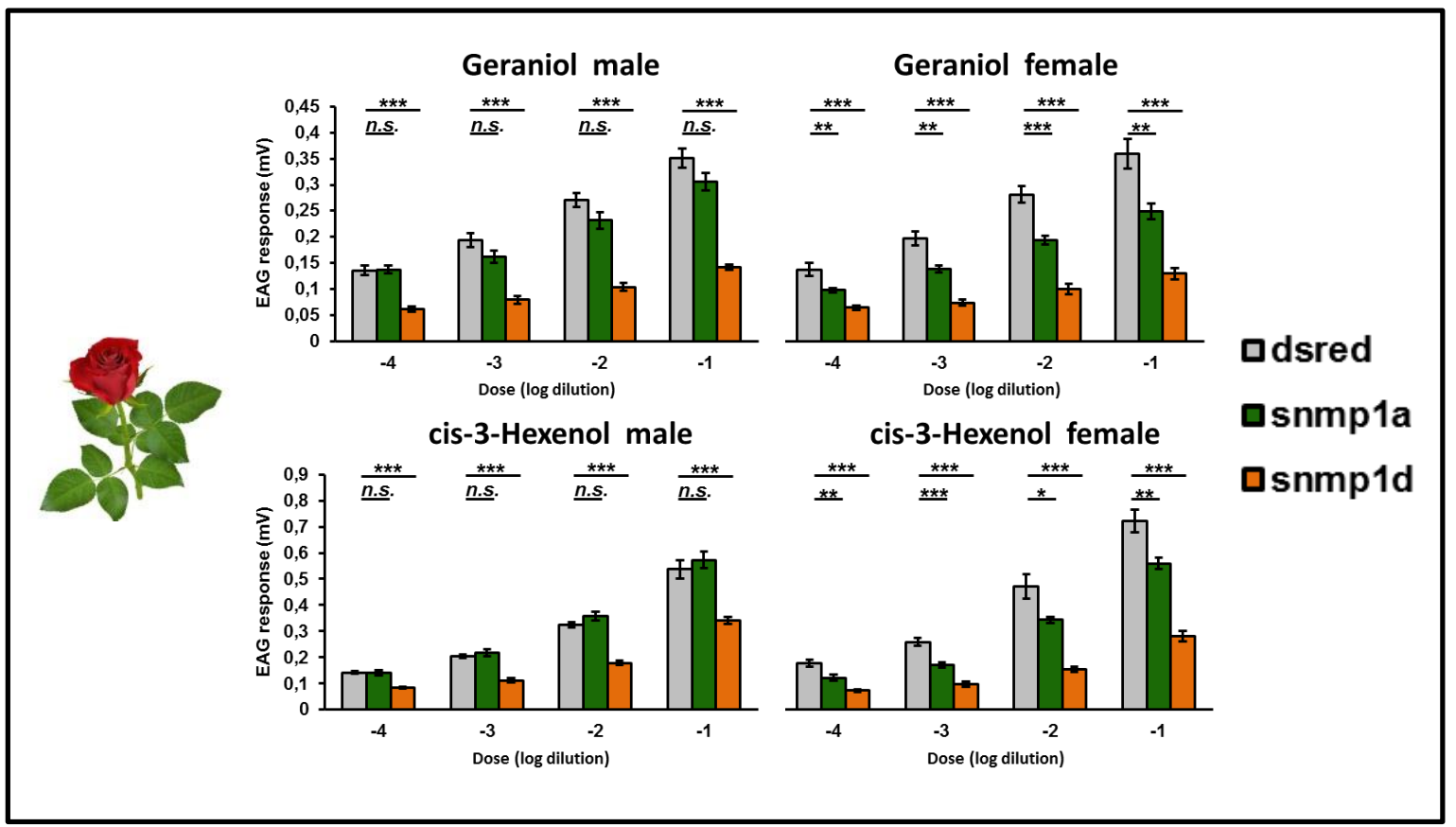

Figure 4.16 EAG responses of RNAi treated T. castaneum to plant odors

Shown are EAG responses of indicated beetles $(n=7)$ and odor dilutions, plotted as mean and s.e.m (standard error of the mean). EAG responses were measured by Karthi Balakrishnan (Forest 
Zoology and Forest Conservation, University Göttingen), after application of geraniol and cis-3hexenol diluted in silicon oil. The asterisks indicate statistically significant differences between control beetles that were injected with dsRNA against DsRed (dsred, grey bars) and knockdown beetles injected with dsRNA against TcSNMP1a (snmp1a, green bar) or TcSNMP1d (snmp1d, yellow bar), respectively. Statistical significance was calculated using ANOVA with Bonferroni post hoc test: ${ }^{*} p<0.05{ }^{* *} p<0.01 ;{ }^{* * *} p<0.001 ;$ n.s. no significant difference. Line ends indicate experimental groups showing significant differences as indicated.

\subsubsection{Validation of the RNAi-induced knockdown by FISH}

To confirm that dsRNA-injection against a given SNMP led to knock-down of the respective transcript, FISH was conducted after the EAG measurements. For that purpose, a digoxigenin-labelled SNMP1d probe was combined with a biotinlabelled probe targeting SNMP1a transcripts. In the representative eleventh segment of control beetles that were injected with dsRNA targeting DsRed (figure 4.17, upper row), SNMP1d transcripts (figure $4.17 \mathrm{~A}$ ) as well as $S N M P 1 a$ transcripts (figure $4.17 \mathrm{~B}$ ) were visualized. This confirms that dsRNA targeting DsRed has no influence on the transcripts of TcSNMP1a and TcSNMP1d, showing that this control experiment is suitable.

In beetles that were injected with dsRNA against transcripts of SNMP1a (figure 4.17, middle row) or SNMP1d (figure 4.17, bottom row), respectively, only the transcripts were hybridized and visualized by the FISH probe detecting the nonRNAi-targeted transcript (figure 4.17). Consequentially no hybridization signals were obtained of the RNAi targeted transcripts (figure 4.17). This indicates that the transcript levels of these genes were reduced to such an extent, that they were below the detection limit of FISH. Taken together, FISH showed that transcripts of SNMP1d as well as SNMP1a in OSNs of the control beetles were unaffected. Furthermore, the knock-down of SNMP1a and SNMP1d was verified. 


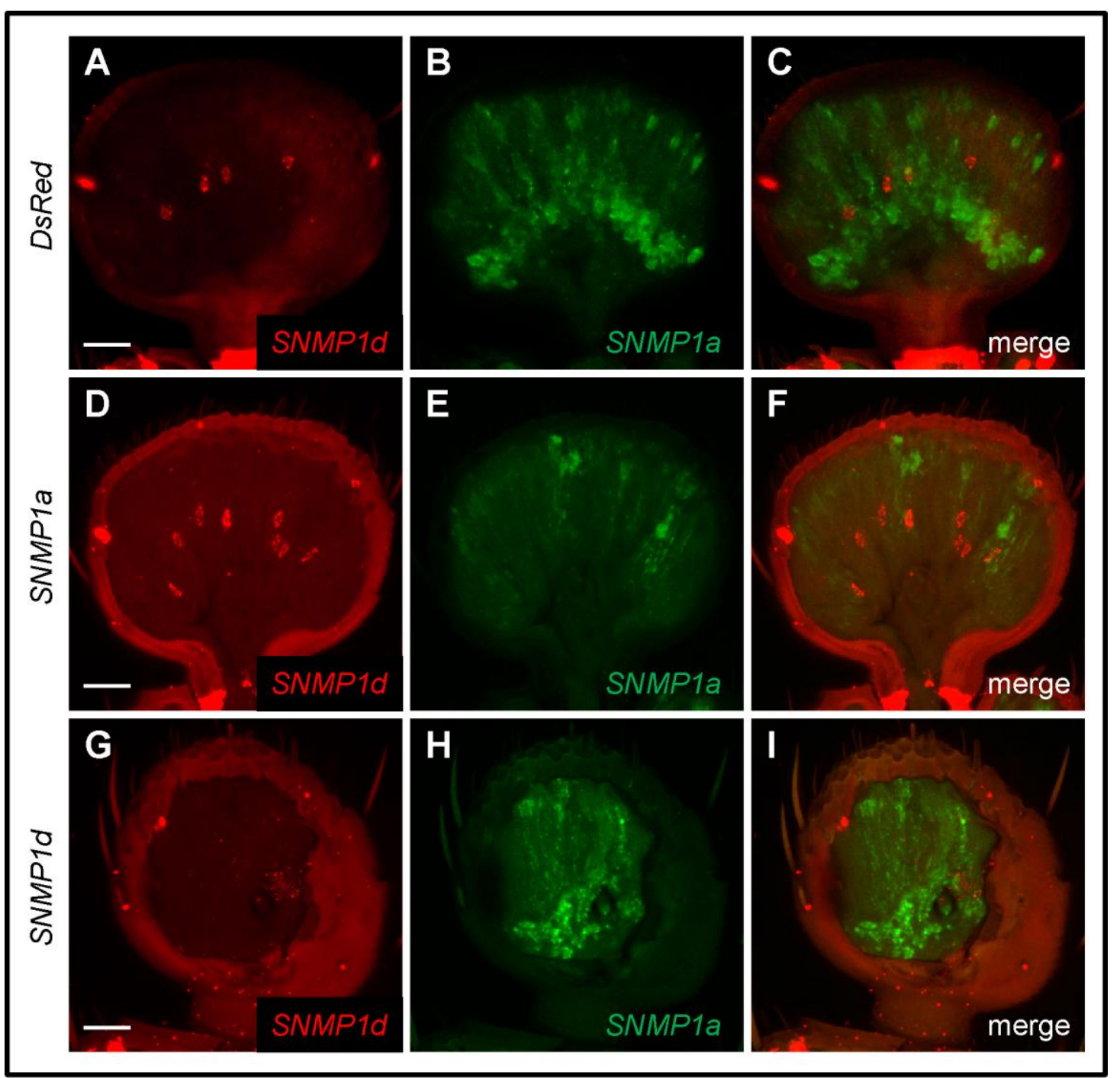

Figure 4.17 Validation of the RNAi-induced knockdown

Shown are projected image-stacks of the antennal segment 11 of $T$. castaneum, injected with dsRNA targeting transcripts as indicated on the left side. FISH was performed with a digoxigenin-labelled probe targeting SNMP1d transcripts and a biotin-labelled probe targeting SNMP1a transcripts. Transcripts were visualized by the HNPP/FastRed detection system for digoxigenin-labelled probes (red fluorescence, A, D, G) followed by the TSA detection system for biotin-labelled probes (green fluorescence, B, E, H). The separate fluorescence channels (A, D, G, B, E, H) and the overlay of both fluorescence channels are shown (C, F, I). Scale bar is $20 \mu \mathrm{m}$. 


\subsection{Analysis of the conservation of SNMPs}

SNMPs play a crucial role for the pheromone detection in insects (Benton et al., 2007; Jin et al., 2008; Li et al., 2014). Flies of the species D. melanogaster that lack a functional SNMP1 are not able to detect the fly aggregation pheromone cisvaccenyl acetate (cVA) anymore (Benton et al., 2007; Jin et al., 2008). Despite the importance of this protein class, the functional mechanism of these proteins is not clarified yet. However, it was shown that this mechanism is highly conserved. When heterologously expressed in D. melanogaster OSNs, the pheromone receptor of the moths Heliothis virescens and Bombyx mori are completely functional and responsive to their cognate pheromone (Kurtovic et al., 2007). In later studies it was shown that this functionality is dependent on the presence of DmSNMP1 (Benton et al., 2007; Li et al., 2014). Although the tested moth pheromone receptors are narrowly tuned to their respective pheromone, the DmSNMP1 is functionally conserved. More precisely, DmSNMP1 could deal with all tested pheromones independently of which species it was derived from (Benton et al., 2007; Li et al., 2014).

In the present study, it was tested, whether SNMPs are generally conserved regarding their function. For that purpose, the responses of pheromone reactive neurons of D. melanogaster expressing different SNMPs instead of the endogenous DmSNMP1 were measured after application of the fly pheromone cVA using twophoton calcium imaging. To this end, flies were generated with different genetic features. Except for the wild type control, all investigated flies were in a transheterozygotic snmp1 mutant background, resulting in complete loss of the endogenous DmSNMP1 function. Furthermore, in all measured flies GAL4 expression was driven by the endogenous DISNMP1 driver, leading to GAL4 expression in the DmSNMP1 expression pattern. GAL4 was used to activate two responder genes in one given fly, namely the $S N M P$ of interest and the calcium sensor GCaMP5G, which enabled calcium imaging. Two-photon calcium imaging was performed in cooperation with the laboratory of Prof. André Fiala (Molecular Neurobiology of Behaviour, University Göttingen). This method allowed to measure neuronal activity of glomeruli in the antennal lobe of D. melanogaster. After detection of an odorant, the cellular calcium level increases and this is 
Results

detectable by an increasing fluorescence of the calcium sensor GCaMP5G (Akerboom et al., 2012). For this study, the activity of the corresponding glomerulus of the cVA detecting neurons, namely glomerulus DA1, was particularly interesting (Vosshall et al., 1999). First, the functionality of this rescue assay system had to be verified. For that purpose, measurements of wild type flies, snmp1 mutant flies, and snmp1 mutant flies rescued by expression of the endogenous DmSNMP1 transgene after application of cVA were conducted and compared.

The representative antennal lobes of the different genotypes comprising the various glomeruli were distinctly recognizable due to expression of the calcium sensor GCamP5G, resulting in a visible basal fluorescence (figure $4.18 \mathrm{~A}$, upper row). In wild type flies and in flies that carry the endogenous DmSNMP1 to rescue the snmp1 mutant situation, cVA application led to an raising calcium level in the OSNs of the DA1 glomeruli, detectable by an increasing fluorescence (figure $4.18 \mathrm{~A}$, lower row). In contrast to that, the DA1 glomerulus of the snmp1 mutant fly did not react to $\mathrm{cVA}$ and as a consequence no increase of cellular calcium was detectable (figure $4.18 \mathrm{~A}$, lower row).

Five independent flies of each genotype were measured and the obtained curves, representing the relative fluorescence change over time, were averaged. In snmp1 mutant flies no cVA induced response was detectable (figure $4.18 \mathrm{~B}$, blue line). In contrast, pheromone sensitive neurons of flies with a wild type DmSNMP1 allele and of flies expressing the endogenous DmSNMP1 transgene in the snmp1 mutant background (DmSNMP1 rescue flies) responded to cVA (figure $4.18 \mathrm{~B}$, black and green curve). The curve progression of wild type and DmSNMP1 rescue flies was almost identical (figure $4.18 \mathrm{~B}$, black and green curve). This shows that the DmSNMP1 transgene fully restored the lack of endogenous DmSNMP1 function with regard to response kinetics and maximal fluorescence change. The comparability of both situations was confirmed by statistical analysis of the maximal fluorescence change (figure $4.18 \mathrm{C}$ ). Taken together, the results showed that the rescue assay system is an appropriate approach to analyze different SNMPs for conserved or adapted functions by testing their rescue abilities. 


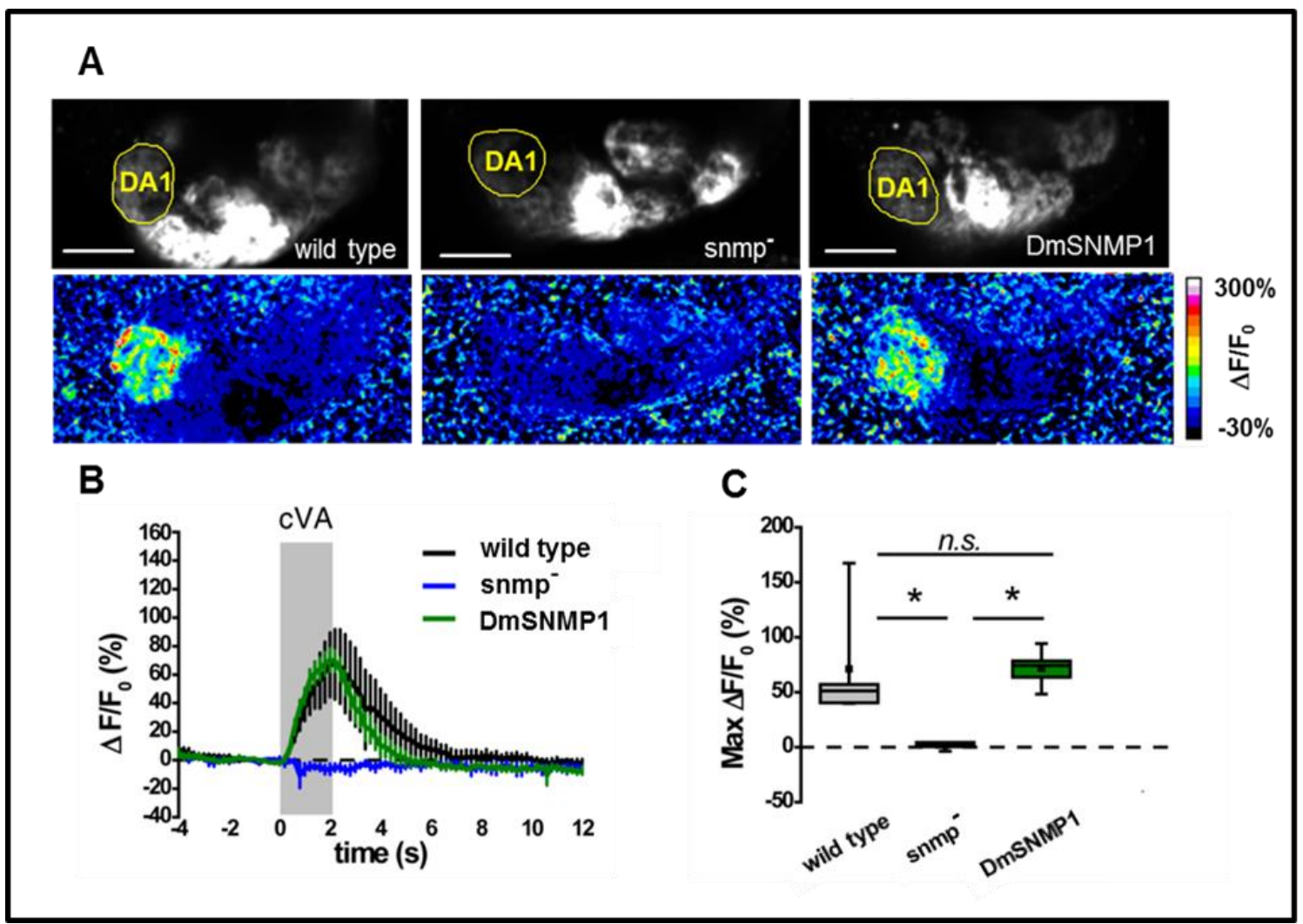

Figure 4.18 Functionality of the $D$. melanogaster rescue assay system

In vivo two-photon calcium imaging of the antennal lobe of D. melanogaster during cVA application (cVA diluted 1:10 in mineral oil). A: The upper row illustrates the prepared left antennal lobes of flies with the indicated genotype. Wild type: third chromosome is wild type with endogenous DmSNMP1 gene; snmp-: transheterozygotic snmp1 mutant resulting in complete loss of functional DmSNMP1; DmSNMP1: flies expressing DmSNMP1 under the control of the DmSNMP1-Gal4 transgene in a snmp1 mutant background. All flies bearing the SNMP-Gal4 transgene and the UASGCaMP5G transgene. The DA1 glomerulus is highlighted by a yellow circle. Scale bar is $20 \mu \mathrm{m}$. The false color pictures below show the quantification of the increasing fluorescence as response to cVA application. B: Averaged curves of 5 flies per genotype obtained from two-photon calcium imaging of the DA1 glomerulus. The grey bar indicates the duration of the stimulus delivery. C: Maximal measured calcium level of five flies per genotype were plotted as box plot and s.e.m (standard error of the mean). The asterisks indicate statistically significant differences $\left({ }^{*} \mathrm{p}<0.05\right)$, calculated using ANOVA with post hoc Bonferroni correction. n.s. no significant difference. 
Results

\subsubsection{TcSNMPs do not rescue DmSNMP1 loss-of-function in D. melanogaster}

The DmSNMP1 homolog of D. melanogaster is able to mediate the detection of cVA and pheromones of different Lepidoptera, which implicates a very specialized and conserved function (Benton et al., 2007; Li et al., 2014). To test whether the diversified TcSNMPs have the same conserved properties and function as the DmSNMP1, the rescue assay system (chapter 4.4, figure 4.18) was applied. For all tested SNMPs, at least five independent flies were measured. The siblings of the respective rescue flies that did not carry the rescue transgene served as snmp1 mutant control (snmp-).

The relative fluorescence change of the DA1 glomerulus before, during and after cVA application revealed that the wild type flies respond strongly to the fly aggregation pheromone, whereas the snmp1 mutant flies (snmp-) cannot detect this odor (figure 4.19 A). Flies expressing a TcSNMP did not show neuronal activity after cVA application, consequentially no fluorescence change was detectable (figure 4.19 A). Statistical analysis of the measured maximal fluorescence changes confirmed the results of the obtained curves (figure $4.19 \mathrm{~B}$ ), by showing that there is no significant difference between the snmp1 mutant situation and the different TCSNMP rescue situations. In summary, the experiments revealed that the diversified TcSNMPs of T. castaneum show not the conserved function described for DmSNMP1. This hints to an adapted function of the TcSNMPs. 


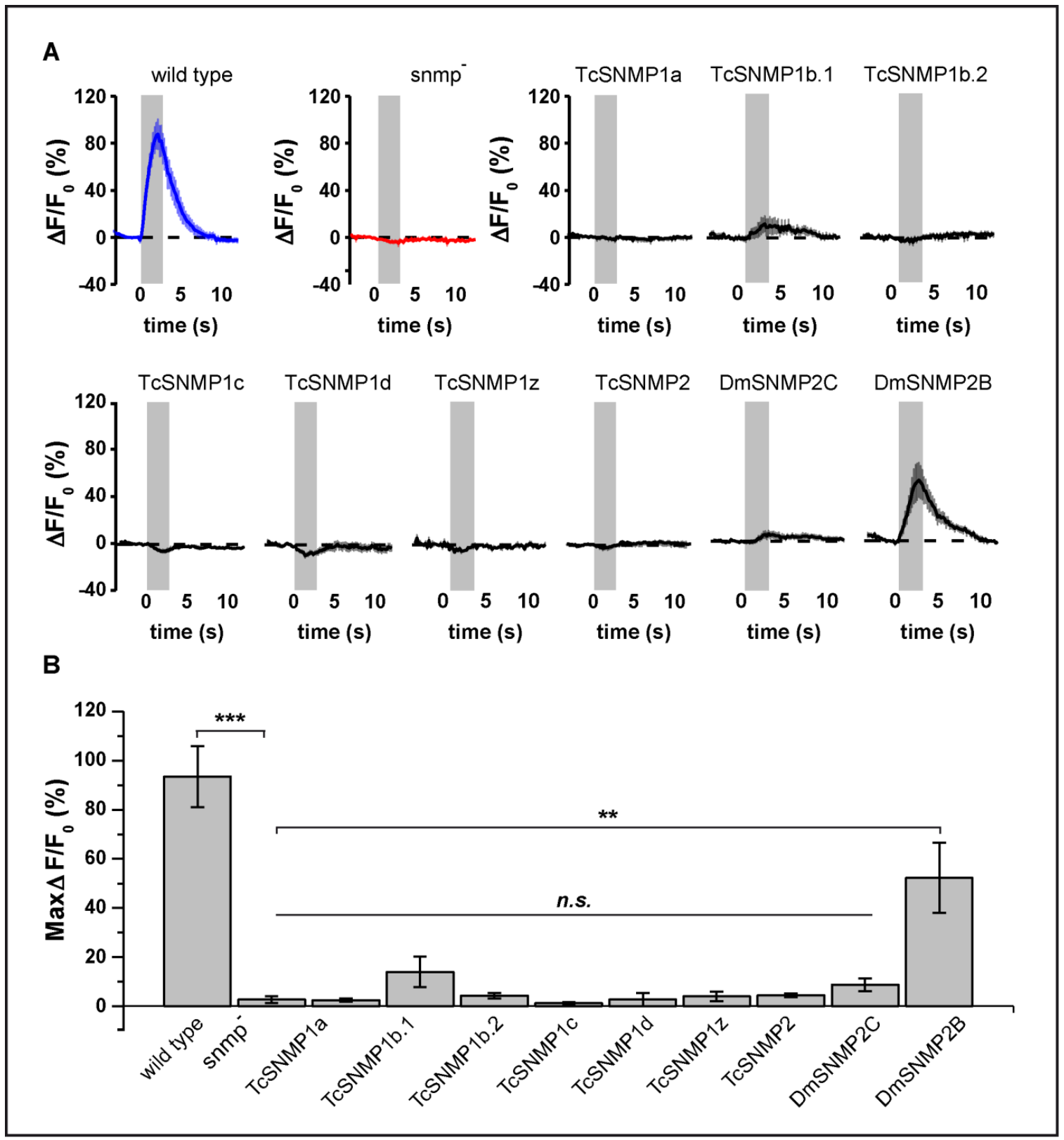

Figure 4.19 Rescue abilities of different SNMPs

In vivo two-photon calcium imaging of the DA1 glomerulus within the antennal lobe of $D$. melanogaster during cVA application (cVA diluted 1:10 in mineral oil).

A: Fluorescence change in percent indicating the cellular calcium level before, during and after cVA application illustrated as averaged curves of at least 5 flies per indicated genotype. Wild type: third chromosome is wild type with endogenous DmSNMP1 gene; snmp-: transheterozygotic snmp1 mutant resulting in complete loss of functional DmSNMP1, other: flies expressing the gene specified above the curve under the control of the SNMP1-Gal4 transgene in a snmp1 mutant background. All flies bear the SNMP-Gal4 transgene and the UAS-GCaMP5G transgene. The grey bar indicates the duration of the cVA delivery. B: Maximal measured fluorescence change of at least five flies per genotype was plotted as mean and s.e.m. The asterisks indicate statistically significant differences $\left({ }^{* *} \mathrm{p}<0.01 ;{ }^{* * *} \mathrm{p}<0.001\right)$ calculated using ANOVA with post hoc Bonferroni correction. n.s. no significant difference. Bracket ends indicate experimental groups showing significant differences. Lines show the statistical difference for all groups below. 
Results

\subsubsection{TcSNMPs modify the odor response profile of the DA1 glomerulus of $D$. melanogaster}

DmSNMP1 is not only expressed in neurons expressing the cVA sensitive odorant receptor 0R67d but also in neurons that are responsible for the detection of other odorants (Benton et al., 2007; Hallem and Carlson, 2006; Ronderos et al., 2014). Among other odors, 1-hexanol and isoamyl acetate are also detected by DmSNMP1 expressing neurons (Hallem and Carlson, 2006; Hallem et al., 2004). To test whether the response to these odors is also influenced or even absent in a DmSNMP1 loss-of-function situation and whether other SNMPs do influence the detection of these substances, the described rescue assay system was applied (chapter 4.4, figure 4.18). For that purpose, two glomeruli, that react to 1-hexanol and isoamyl acetate, were measured and evaluated: DC1 and DM6 (Hallem and Carlson, 2006; Hallem et al., 2004). The procedure of the measurements was conducted as described for the cVA measurements (chapter 4.4.1). These measurements revealed that the detection of 1-hexanol and isoamyl acetate is independent of the presence of DmSNMP1 and is also not influenced by the expression of all the tested SNMPs in the glomeruli DC1 and DM6 (data not shown).

However and most interestingly, the cVA responsive glomerulus DA1 that is normally inhibited by 1-hexanol (van der Goes van Naters and Carlson, 2007) responded to 1-hexanol in flies carrying the TcSNMP2 transgene (figure 4.20 A). The statistical analysis confirmed that the excitation of the TcSNMP2 expressing neurons, indicated by the maximal fluorescence change, is highly significantly different compared to wild type flies (figure 4.20 B).

The DA1 glomeruli of flies carrying the TcSNMP1a transgene or the TcSNMP1b.1 transgene, respectively, also showed a changed response to 1-hexanol: TcSNMP1a or TcSNMP1b.1 expressing neurons showed neither excitation nor inhibition by 1hexanol application (figure 4.20 A). The same was true for odorant stimulation by isoamyl acetate (figure 4.21). This indicates that TcSNMP1a and TcSNMP1b.1 are able to modify the response profile of the investigated DA1 glomerulus towards different odorants. 
In summary, the odors 1-hexanol and isoamyl acetate are detected independently of DmSNMP1 in D. melanogaster. Remarkably, in this artificial situation some TcSNMPs are able to change the odor response profile of the DA1 OSNs towards these odors. Both, the absent inhibition as well as the positive response of the DA1 induced by 1-hexanol suggests that SNMPs have more functions than just the mediation of odors. Unraveling of these unknown functions could explain the diversification of the TcSNMPs and could give new insights into the sensory mechanisms of odor detection in insects.

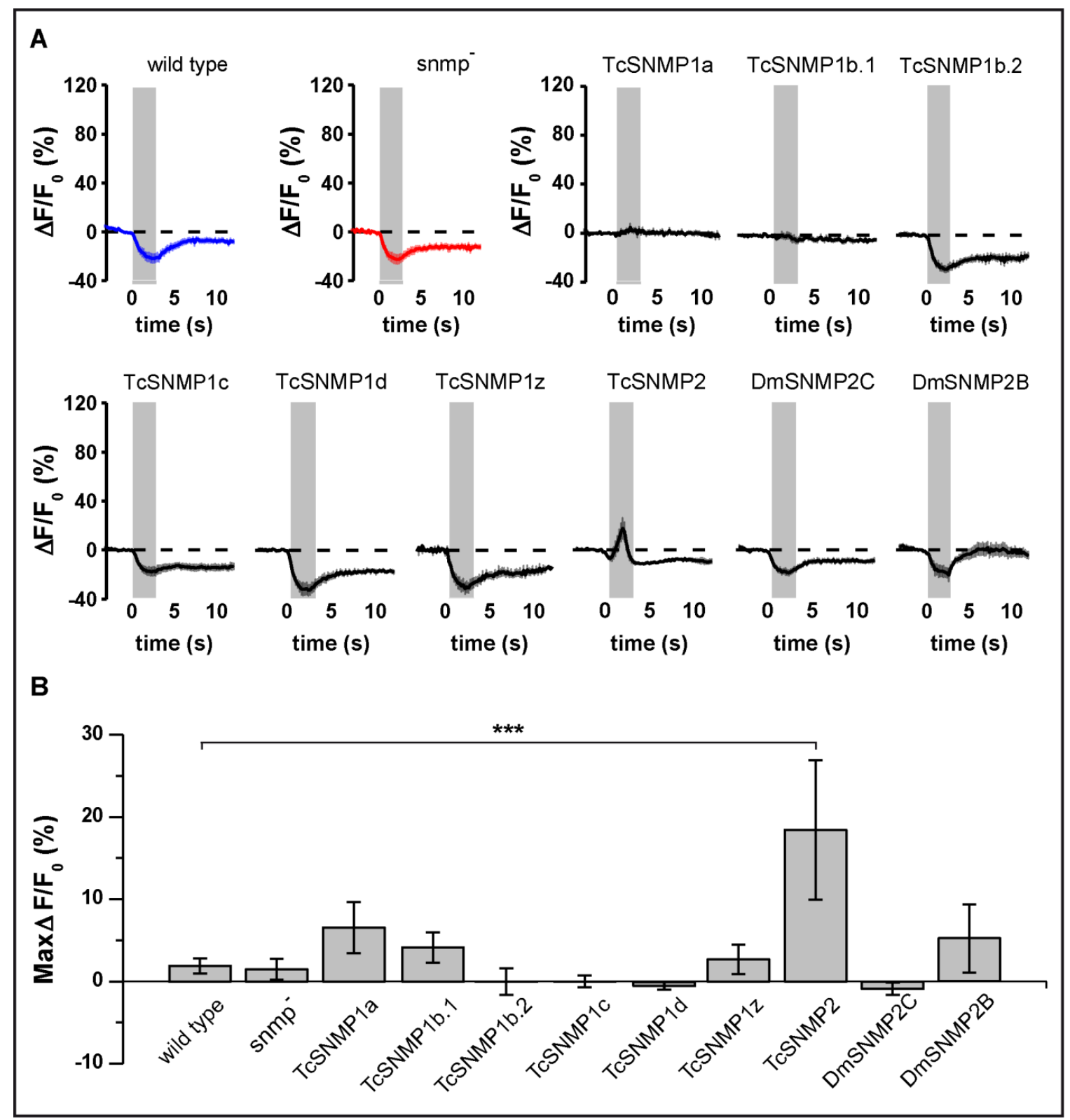

Figure 4.20 Modified odor responses of the DA1 glomerulus to 1-hexanol induced by TcSNMPs 
In vivo two-photon calcium imaging of the DA1 glomerulus within the antennal lobe of $D$. melanogaster during 1-hexanol application (1-hexanol diluted 1:100 in mineral oil).

A: Fluorescence change in percent indicating the cellular calcium level before, during and after 1hexanol application depicted as averaged curves of at least 5 flies per indicated genotype. Wild type: third chromosome is wild type with endogenous DmSNMP1 gene; snmp: : transheterozygotic DmSNMP1 mutant resulting in complete loss of functional DmSNMP1, other: flies expressing the gene specified above the curve under the control of the SNMP1-Gal4 transgene in a snmp1 mutant background. All flies bear the SNMP-Gal4 transgene and the UAS-GCaMP5G transgene. The grey bar indicates the duration of the 1-hexanol delivery. B: Maximal measured calcium level of at least five flies per genotype were plotted as mean and s.e.m. The asterisks indicate statistically significant differences $\left({ }^{* * *} \mathrm{p}<0.001\right)$, calculated using ANOVA with post hoc Bonferroni correction. If not mentioned otherwise no significant difference was calculated when compared to the wild type group. Bracket ends indicate experimental groups showing significant differences.

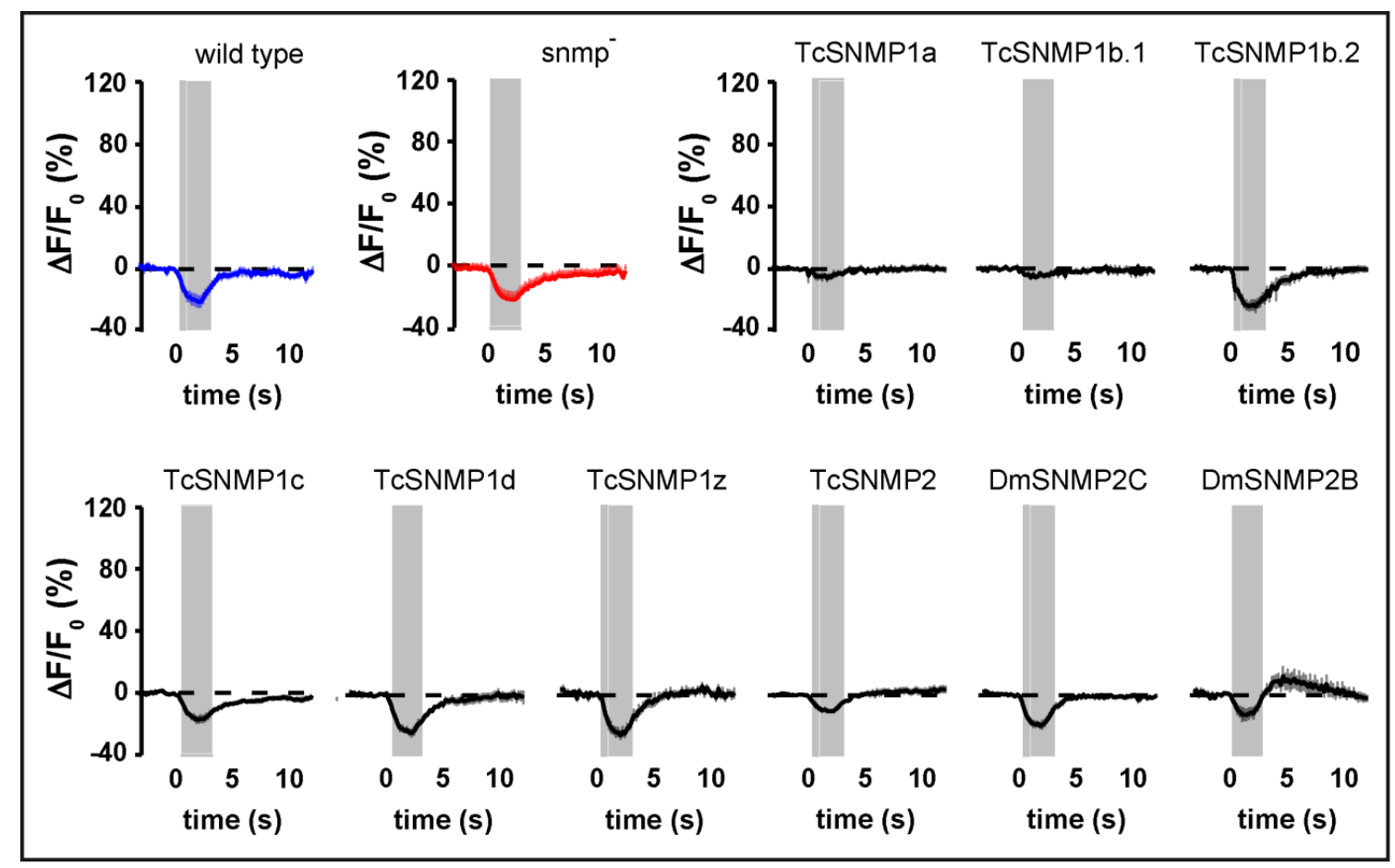

Figure 4.21 Modified odor responses of the DA1 glomerulus to isoamyl acetate induced by TcSNMPs

In vivo two-photon calcium imaging of the DA1 glomerulus within the antennal lobe of $D$. melanogaster after isoamyl acetate application (isoamyl acetate diluted 1:100 in mineral oil).

Fluorescence change in percent indicating the cellular calcium level before, during, and after isoamyl acetate application, depicted as averaged curves of at least 5 flies per indicated genotype. Wild type: third chromosome is wild type with endogenous DmSNMP1 gene; snmp: transheterozygotic snmp1 mutant resulting in complete loss of functional DmSNMP1, other: flies expressing the gene specified above the curve under the control of the SNMP1-Gal4 transgene in a snmp1 mutant background. All flies bearing the SNMP-Gal4 transgene and the UAS-GCaMP5G transgene. The grey bar indicates the duration of the isoamyl acetate delivery. 


\subsubsection{DmSNMP2B rescues DmSNMP1 loss-of-function in D. melanogaster}

In addition to the TcSNMPs, also DmSNMP2 was tested for its rescue abilities by using the rescue assay system (chapter 4.4, figure 4.18). DmSNMP2 occurs in two isoforms (DmSNMP2B/C) and is mainly expressed in the body, where it fulfills different functions besides of olfaction (Herboso et al., 2011). Therefore, it was interesting to investigate whether ectopically expressed DmSNMP2 is able to fulfill the functional task of DmSNMP1 in antennae of snmp1 mutant flies. Thus, DmSNMP2 was analyzed to clarify, whether these two D. melanogaster SNMPs might be functionally conserved despite their different tissue-specific expression. Analogously to the investigated TcSNMPs, at least five independent flies carrying the rescue transgene DmSNMP2B or DmSNMP2C, respectively, were measured. The siblings of the respective rescue flies that did not carry the rescue transgene served as snmp1 mutant control (snmp-).

Remarkably, the isoform B of DmSNMP2 (DmSNMP2B) was able to complement the lack of DmSNMP1 function (figure 4.19 A), whereas the DmSNMP2 isoform C (DmSNMP2C) was not (figure $4.19 \mathrm{~A}$ ). Comparison of the measured maximal fluorescence changes with subsequent statistical analyses confirmed that only the $D m S N M P 2 B$ rescue is significantly different to the snmp1 mutant situation (figure 4.19 B). However, comparison of the cVA response of wild type flies and DmSNMP2B rescue flies showed a decreased fluorescence change of the DmSNMP2B rescue flies, indicating a reduced functionality.

To verify these results, the measurements were repeated with additional snmp1 mutant flies carrying the DmSNMP2B or DmSNMP2C rescue construct, respectively. Here, the same results were obtained as previously (data not shown), meaning that the observations are reproducible. After these measurements, the identity of the heterologously expressed transgene of the measured flies was analyzed and confirmed by PCR (data not shown). Taken together, the results showed that the DmSNMPs of D. melanogaster are partially conserved with regard to their function. Interestingly, only isoform B of DmSNMP2 was able to rescue the lack of DmSNMP1 function, but in a reduced extend.

Remarkably, DmSNMP2C has one additional exon compared to DmSNMP2B. This suggests that the absence of this exon connects two parts of the protein to a 
Results

putative functional region that is able to complement lacking DmSNMP1 function. However, no detailed information about DmSNMP protein-structures are available yet. To get more insights into the putative functional region of the DmSNMP2B protein in comparison with the DmSNMP2C protein, as well as the corresponding regions in the DmSNMP1 protein, a structure prediction was performed using the Phyre2 software (Kelley et al., 2015). The CD36 protein LIMP-II (lysosome membrane protein 2) was used as the main homology template. Additional other proteins such as n-myristoyltransferase from Leishmania donovani or human ephrin type-a receptor 2 were included into the calculation. The calculated tertiary protein structures were illustrated by the PyMOL Molecular Graphics System (Version 1.7.4 Schrödinger, LLC; figure 4.22). The region of DmSNMP2B (figure $4.22 \mathrm{~A}$ ) that is disconnected by the additional exon of DmSNMP2C (figure 4.22 B, yellow colorized) represents a putative alpha helix (figure 4.22 B, red colorized). This suggests that the functional area of DmSNMP2B that is able to rescue the snmp1 mutant effect might be located in this helix. At the respective position, a similar helix is also located in DmSNMP1 (figure 4.22 C, white colorized). In order to characterize the functional area of DmSNMP1 in more detail it could be promising to focus on this helix in further analyses. 


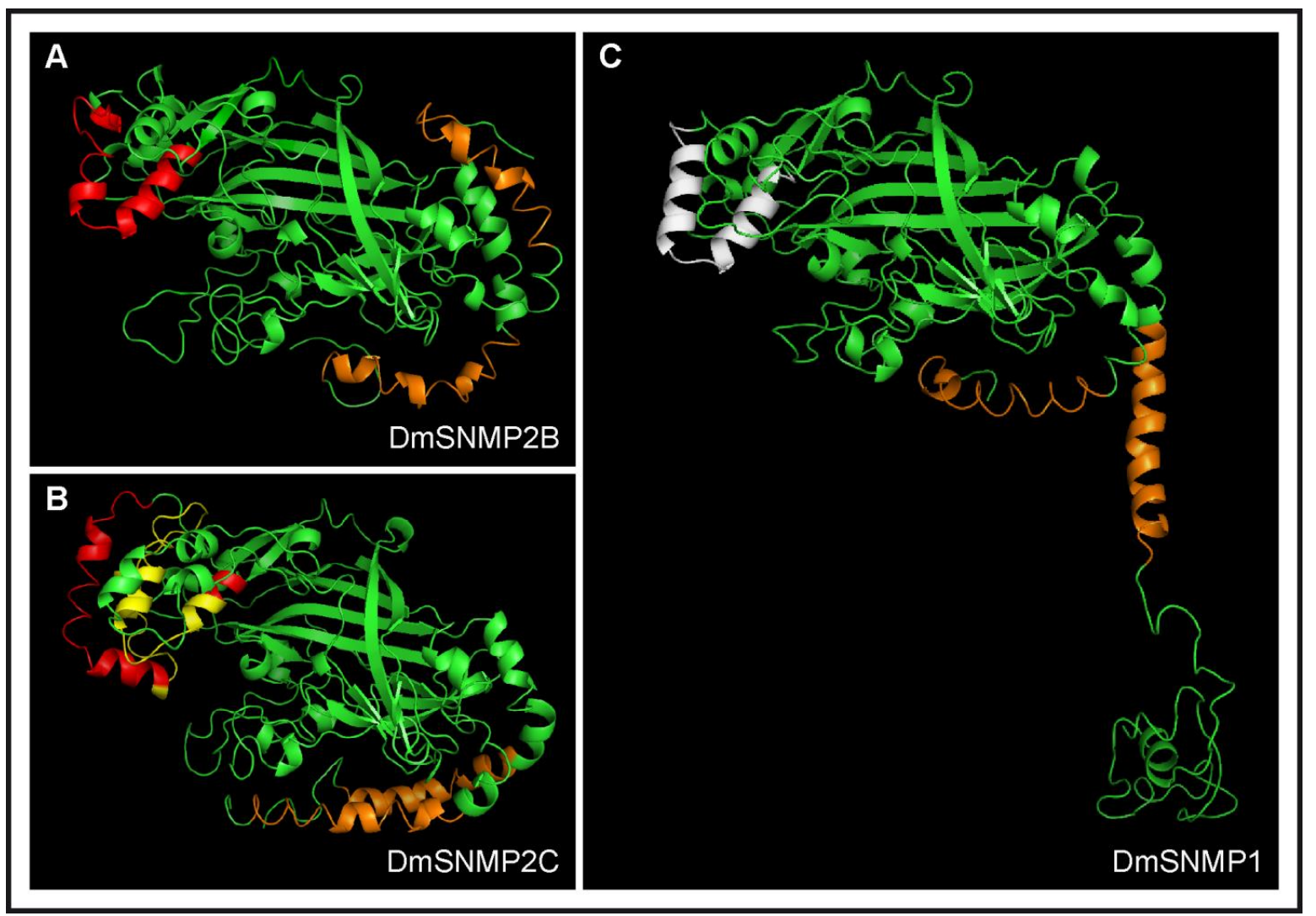

Figure 4.22 Structure prediction of different SNMPs of $D$. melanogaster

Several templates such as the CD36 protein LIMP-II (lysosome membrane protein 2) were used to predict the tertiary structure of indicated D. melanogaster SNMPs using Phyre2 prediction server (Kelley and Sternberg, 2009; Kelley et al., 2015). Illustration was conducted using PyMOL Molecular Graphics System (version 1.7.4 Schrödinger, LLC). TMDs are shown in orange.

A: Structure prediction of DmSNMP2 Isoform B (DmSNMP2B). 413 amino acid residues (81\%) were modelled at $>90 \%$ accuracy. The red visualized sequence represents the alpha helical region of interest that is connected by absence of the additional exon. This absence leads to the ability to rescue the snmp1 mutant effect.

B: Structure prediction of DmSNMP2 Isoform C (DmSNMP2C). 405 amino acid residues (73\%) were modelled at $>90 \%$ accuracy. The red visualized sequence represents the alpha helical region of interest. The additional exon that disconnects the red visualized alpha helix in this region is shown in yellow.

C: Structure prediction of DmSNMP1. 396 amino acid residues (72\%) were modelled at >90\% accuracy. The white visualized sequence represents the corresponding alpha helical region of interest. 


\section{Discussion}

\subsection{The organization of an olfactory sensillum of $\boldsymbol{T}$. castaneum}

In this study a FISH protocol for antennae of T. castaneum was established (chapters 4.2.1 and 4.2.2). Thereby, it was revealed that the cell bodies of the OSNs are located proximal within the segment and distant from the segment's distal cuticle, from which the olfactory sensilla project outwards. The OBP expressing supporting cells are located in a more distal layer within an antennal segment (figure 4.7). Such an arrangement in layers had already been described for single sensilla basiconica of T. castaneum (Roth and Willis, 1951) and was thus confirmed by the findings using FISH. However, this arrangement is different to the described model of the cellular organization of olfactory sensilla in other insects (Keil and Steinbrecht, 1984; Shields, 2008). In antenna of various insects, like $D$. melanogaster, the kissing bug Triatoma infestans, or B. mori it has been shown that the cell bodies of the OSNs that are enwrapped by supporting cells are located directly beneath the cuticle forming the olfactory sensilla (Diehl et al., 2003; Gnatzy et al., 1984; Kim and Smith, 2001; Shanbhag et al., 2000).

The supporting cells belong to three different types and fulfill different tasks. The trichogen cells and tormogen cells are believed to produce and secrete the sensillar lymph with its components into the sensilla hair (Gnatzy et al., 1984; Kim and Smith, 2001). In order to do so, the tormogen cell is tightly connected with the adjacent cuticle. The supporting cells visualized by the probe targeting OBP transcripts in antenna of T. castaneum were most likely tormogen or trichogen cells, given that they are producing a secreted OBP. The third type of supporting cells, the thecogen cells, are proposed to build a sheath around the cell body of the neuron (Gnatzy et al., 1984). Interestingly, in mechanosensitive sensilla of insects it has been shown that the thecogen cells also ensheath the cell body and dendrites of the respective neurons (Keil, 1997). Already during development, it secretes the dendrite sheath to enclose the dendrite with a thin layer (Akai and King, 2012). 
Because of this specific role, this kind of supporting cell is unlikely located distally to the cell bodies of the olfactory sensory neurons.

Assuming that T. castaneum has the same kind of supporting cells and that they fulfill the same functions as described for other insects, the obtained FISH stainings of transcripts in OSNs or supporting cells (figure 4.7) led to a potential model of an olfactory sensillum of T. castaneum (figure 5.1). This model has the same cell types as described for other insects (Keil and Steinbrecht, 1984), but the cell bodies of the secretory supporting cells are located in a more distal layer compared to the cell body of the OSN with the ensheathing thecogen cell. The latter ones are located proximal within a segment, distant to the olfactory sensillum.

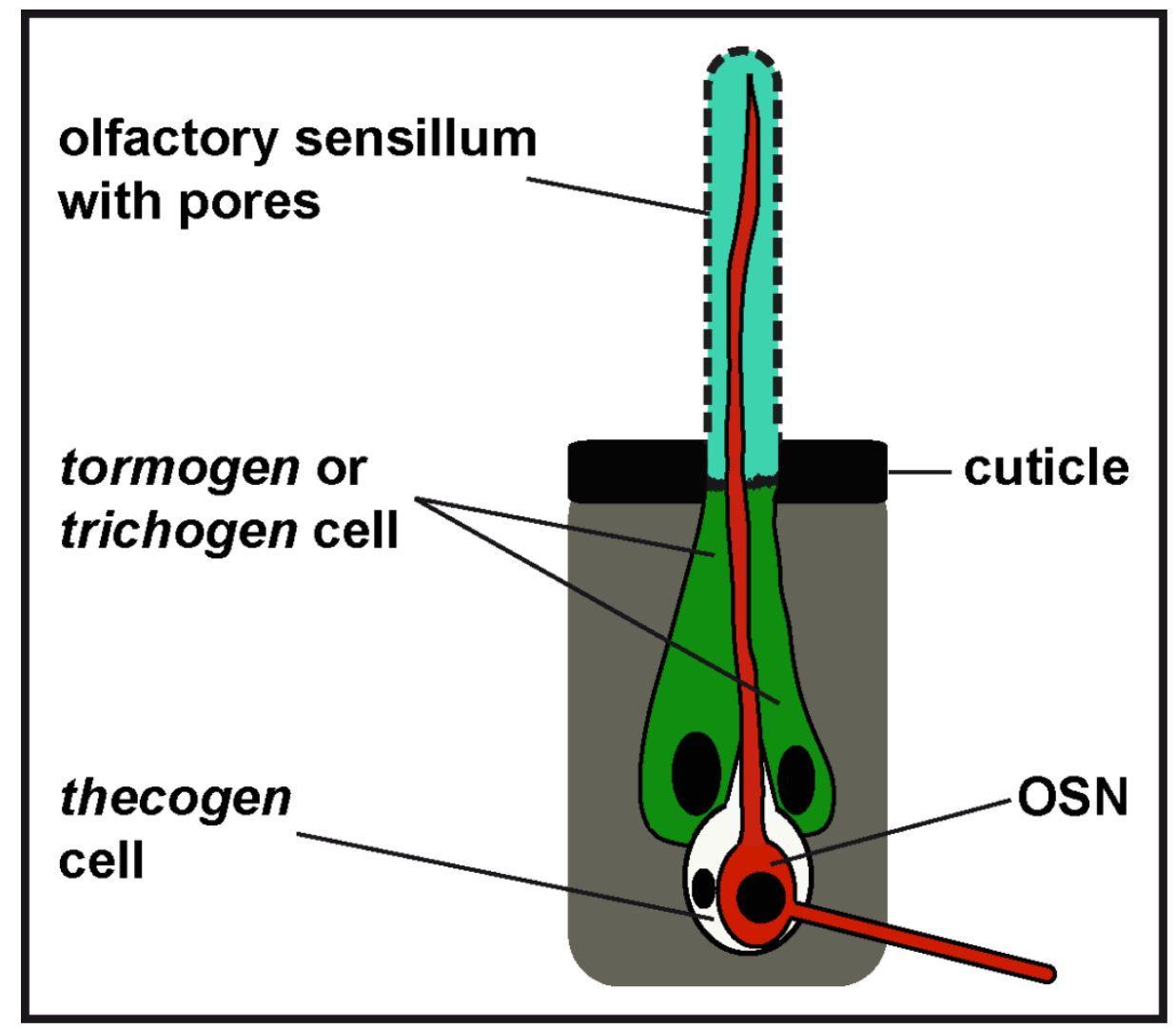

Figure 5.1 Model of an olfactory sensillum of T. castaneum

The obtained FISH signals by using probes targeting transcripts of OSNs or supporting cells as well as the assumption that $T$. castaneum antenna have the same cell types as indicated for other insects led to this model. Shown is the potential arrangement of indicated supporting cells and the olfactory sensory neuron (OSN) under an olfactory sensillum of T. castaneum.

Further experiments such as transmission electron microscopy will help to determine the localization of the different kinds of supporting cells and thereby test this model for correctness. Unfortunately, first approaches to investigate the 
Discussion

cellular structure within antennae of $T$. castaneum by transmission electron microscopy were not successful. A comprehensive set of available fixation approaches have been tested, but the cuticle of this beetle appears to be impermeable to all of them. For future approaches other fixation possibilities have to be tried out. Cryofixation by high pressure freezing or self-pressurized rapid freezing might be feasible (Leunissen and Yi, 2009; Moor, 1987).

\subsection{Identification of six SNMPs in T. castaneum}

OSNs of insects gain their function to detect odorants by a characteristic set of proteins: an odor-specific OR and the coreceptor Orco (Leal, 2013; Vosshall and Hansson, 2011). For the detection of hydrophobic pheromones a further receptor is needed, the CD36-related SNMP (Benton et al., 2007; Jin et al., 2008; Kurtovic et al., 2007). During annotation of the genome of the red flour beetle T. castaneum, seven genes were found, that could encode for SNMP homologs (Nichols and Vogt, 2008; Vogt et al., 2009). The antennal expression of six of the seven annotated SNMPs was verified in this study by RACE PCR (chapter 4.1.1). Generally just one SNMP1 and SNMP2 homolog each are described to be expressed in different insects of the order Diptera and Lepidoptera, therefore the high number of SNMP genes in T. castaneum was a novel and intriguing finding (Forstner et al., 2008; Nichols and Vogt, 2008; Vogt et al., 2009). This high number of SNMPs in T. castaneum raises the question whether insects of the order Coleoptera have in general more SNMPs. Recent transcriptome analysis of other coleopteran species showed that in the japanese pine sawyer Monochamus alternatus just one SNMP was found whereas in its parasitoid Dastarcus helophoroides four different SNMPs were identified (Wang et al., 2014). Here, it was not stated, whether these SNMPs are homologs of SNMP1 genes or SNMP2 genes. In the yellow mealworm Tenebrio molitor, a beetle within the same family (Tenebrionidae) as T. castaneum, one SNMP1 and SNMP2 homolog were identified (Liu et al., 2015), and in two tree killing bark beetles, Ips typographus and Dendroctonus ponderosae three SNMPS (two SNMP1 homologs and one SNMP2 homolog) were identified (Andersson et al., 2013). This indicates that different coleopteran show a diverse number of SNMP 
homologs and suggests that it is not a common rule that Coleoptera have more SNMPs, but that some beetles, like T. castaneum, have a special use for more SNMPs.

Also in other insect orders exceptions were found that have more SNMPs. In this respect it is intriguing that analysis of the hessian fly Mayetiola destructor transcriptome revealed the expression of seven MdSNMPS, six SNMP1 homologs and one SNMP2 homolog (Andersson et al., 2014). This fly feeds on grain and is known as wheat pest (Shukle, 2008; White and Lambkin, 1988). This means that both insects with the highest known number of expressed SNMPs, T. castaneum and $M$. destructor, live in a similar environmental background. Living in this environment might be an explanation for the need of more SNMPs. Loss of function experiments of the diversified SNMPs of T. castaneum (this study, discussed below) and M. destructor will reveal, whether these SNMPs are involved in the detection of odors that are emitted by typical environmental substances.

\subsection{TcSNMP1z is dissimilar to TcSNMP1 and TcSNMP2 homologs}

RACE-PCR allowed the identification of the actual full length SNMP sequences of T. castaneum (chapter 4.1.1). The identified transcripts were used to determine the putative amino acid sequences of the different TcSNMPs (figure 4.2). In the moth species tobacco budworm Heliothis virescens, tobacco hornworm Manduca sexta and the silk moth Antheraea polyphemus it has been shown that SNMP proteins are highly conserved regarding their amino acid sequences. The investigated moth SNMP1 proteins showed amino acid identities ranging from $66 \%-90 \%$ and the moth SNMP2 proteins shared $67 \%$ - $72 \%$ amino acids (Forstner et al., 2008). In contrast to that, SNMP2 proteins in moth just showed an identity of $26-27 \%$ when compared to the SNMP1 proteins. This is the reason for the different nomenclature, indicating that SNMP2 proteins are not that similar to SNMP1 proteins as SNMP1 or SNMP2 proteins among themselves (Rogers et al., 2001). Remarkably, when compared to the different TcSNMP1 proteins the TcSNMP1z protein shared just 20-24 \% amino acids (figure 4.3). However, when compared to TcSNMP2, also just $24.5 \%$ amino acids were identical (figure 4.3 ). Taken together, 
Discussion

this means that the protein sequence of TcSNMP1z showed an amino acid identity with low similarity to the TcSNMP1 proteins as well as to the TcSNMP2 protein. Following the previous approach of nomenclature, we therefore gave this TcSNMP the name TcSNMP3, indicating a clear difference to the TcSNMP1 and TcSNMP2 protein sequences in T. castaneum.

\subsection{TCSNMP expressing cells are probably housed in sensilla trichodea and sensilla basiconica}

In the vinegar fly D. melanogaster as well as in different moth species SNMP expressing cells have primarily been found in pheromone sensitive sensilla trichodea (Benton et al., 2007; Rogers et al., 1997). By scanning electron microscopy (SEM) of T. castaneum antenna, it has been shown that the about 88 beetle's $s$. trichodea are restricted to the eleventh segment (Dippel et al. in preparation). This finding goes well with the localization of TcSNMP1d, TcSNMP1b and TcSNMP1z expressing cells in the eleventh segment (chapter 4.2.3) and suggests a connection to s. trichodea. However, in the present study it was also shown that TCSNMP1a and TCSNMP1c are expressed in all three club segments (chapter 4.2.3). This indicates that these TCSNMPs are expressed in cells housed in a different sensillum type. On the segments that contain TcSNMP1c and TcSNMP1a expressing cells two other sensilla types have been described, namely sensilla basiconica and sensilla coeloconica (Dippel et al. in preparation). Here, it has been shown that the s. coeloconica are rare, in these only about twelve neurons are housed in each segment nine and ten. In contrast to that, TcSNMP1a and TcSNMP1c expressing neurons were detectable in a higher number (figures 4.8 and 4.10). Therefore these genes are most likely expressed in cells housed in s. basiconica. This sensilla hair type has been found about 100 times on the club segments (Dippel et al. in preparation). This suggestion could be tested by using antibodies against the respective TcSNMP proteins in immunohistochemical approaches. 


\subsection{TcSNMP2 expressing cells weren't detectable in antennal club segments}

Although antennal TCSNMP2 expression was indicated by transcriptome data (Dippel et al. in preparation) and RACE-PCR (chapter 4.1.1), no TcSNMP2 transcripts were detectable after conducting FISH against TcSNMP2. A reasonable explanation for that could be the low expression level of this gene in antenna, shown by transcriptome analysis (Dippel et al. in preparation). In particular, the low number of TCSNMP2 transcripts in antenna could be below the detection threshold of FISH, since especially an ubiquitous low expression would be very hard to distinguish against background staining. Another possibility could be that TCSNMP2 is not expressed in club segments of the antenna but in the more proximal antennal segments that are not involved in olfaction and therefore were not analyzed in this study. In accordance, transcriptome analysis showed that TCSNMP2 is mainly expressed in the body of T. castaneum (Dippel et al. in preparation). An equivalent expression has been described for the D. melanogaster SNMP2 (Chintapalli et al., 2007). Several roles of the DmSNMP2 homolog in the body of $D$. melanogaster have been indicated by expression patterns (Herboso et al., 2011). For example it has been shown that DmSNMP2 is highly expressed in anterior spiracles, the external tracheal apertures (Herboso et al., 2011). In this tissue spiracular glands are located that are linked to the secretion of lipids to coat the surface of the spiracular plate and branched hairs (Keilin, 1944; Keilin et al., 1935; Rizki, 1956). DmSNMP2 expression in this tissue indicates an involvement in these secretion processes (Herboso et al., 2011). Given that TcSNMP2 is expressed mainly in the body and that beetles have a similar structured respiratory system as D. melanogaster (Crowson, 2013), it is plausible that TcSNMP2 is expressed in similar tissues and is involved in similar tasks as DmSNMP2. FISH targeting TCSNMP2 transcripts in the body of T. castaneum will clarify, whether this assumption is correct. 
Discussion

\subsection{The potential role of TcSNMPs in supporting cells}

Double FISH with a combination of probes targeting an antennal TcSNMP and a marker gene for OSNs or secretory supporting cells, respectively, made it possible to identify the TCSNMP expressing cell types (chapter 4.2.3). Following that approach, it was shown that TCSNMP1a and TcSNMP1d are expressed in OSNs whereas TcSNMP1c, TcSNMP1b, and TcSNMP1z are expressed in supporting cells (figures 4.8 - 4.12). This situation, that the supporting cells express a different SNMP than the OSNs, has also been found in various moth species (Forstner et al., 2008). Here, the pheromone sensitive neurons express the moth SNMP1 homolog and the supporting cells express the moth SNMP2 homolog. The role of SNMPs expressed in supporting cells remains unclear but the occurrence in this non neuronal cell type indicates a task apart from odorant detection. Former studies have suggested that supporting cells regulate the composition of the sensillar lymph (Gnatzy et al., 1984). In this regard, they produce and secrete the lymph but also degrade and absorb proteins of the lymph, such as OBPs (Leal, 2003; Steinbrecht et al., 1992). Supporting cells control the ionic composition of the sensillar lymph and it has been proposed that they remove odorants or its degradation products (Blomquist et al., 2003; Thurm and Küppers, 1980). To manage this task the supporting cell needs to have the ability to absorb substances from the sensillar lymph to clean it. Remarkably, some members of the CD36 protein family are involved in endocytosis processes, like the HDL/LDL receptor or the cholesterol transporter in macrophages (Febbraio and Silverstein, 2007). This makes it plausible that SNMPs in the membrane of supporting cells fulfill a similar function and mediate endocytosis of degraded proteins or odors. Hereby they would facilitate a fast and effective cleaning of the sensillar lymph and therefore guarantee a functional and highly sensitive odorant detection system (Forstner et al., 2008).

This suggestion goes well with observations in D. melanogaster. In this fly it has been shown that the DmSNMP1 is expressed in supporting cells all over the antenna, whereas the DmSNMP1 expressing neurons are only located in a distal lateral neuron population (Benton et al., 2007). Furthermore, identification of the 
TcSNMP expressing cell types in T. castaneum by FISH indicates that there are also more kinds of TcSNMPs expressed in supporting cells than in OSNs.

With regard to the hypothesis that SNMPs in supporting cells are necessary for the cleaning of the sensillar lymph, it is plausible that SNMPs are expressed in many supporting cells, because clean sensillar lymph is the basis for an accurate odor detection system in every olfactory sensillum. In contrast, only special neurons need a functional SNMP for the detection of odorants. Therefore it is convincing that more supporting cells need an SNMP to mediate endocytosis of substances to clean the sensillar lymph.

Interestingly, RACE-PCR on antennal cDNA pools confirmed the presence of transcripts of further CD36 proteins within antennae: Tc015854, Tc014951 Tc015144 Tc014954, and Tc000948 (not shown). In this context a particular interesting candidate is Tc015854, the T. castaneum scavenger receptor class $B$ member 1 (XM_966824.3) that is mainly expressed in antennae and represents an orthologue of the D. melanogaster gene Debris buster (Dbs). Recently it has been shown that Dbs is necessary for the degradation of dendrite debris (Han et al., 2014). It might be possible, that the T. castaneum orthologue that is expressed in antenna, has a similar task and could also take part in clearance processes. FISH against transcripts of this gene will clarify whether this gene is expressed in secretory supporting cells and thereby would support this hypothesis or whether it is expressed in another cell type.

\subsection{TcSNMP1d expression is partially correlated with TcSNMP1z expression}

By double FISH with different TcSNMP probes on longitudinal bisected antennae, it was possible to show that TCSNMP1d expressing neurons and TCSNMP1z expressing supporting cells are located partially in neighbored cells (figure 4.13). In more detail, within the eleventh segment, this colocalization was observed in several instances but in the same segment also non-matching TcSNMP1d or TcSNMP1z expressing cells were detectable (figure 4.13). 
Discussion

Importantly, the bisecting cut was done in random angles for each antenna and thereby likely unequally between different antennae. Depending on the bisection plane, it might be that the neighboring cells have been separated by the bisecting cut. This may have led to the observation that TcSNMP1z expressing supporting cells are only partially colocalized with TcSNMP1d expressing neurons, although they were always located next to each other in the intact antenna. However, given that the observed only partial correlated expression was reproducible, it is more plausible that TCSNMP1z expressing supporting cells are indeed only sometimes neighbored to TcSNMP1d expressing neurons.

Since it has been described that SNMP expressing neurons are always connected to supporting cells that also express an SNMP (Benton et al., 2007; Forstner et al., 2008), I investigated whether another TCSNMP homolog is expressed next to the remaining TcSNMP1d expressing neurons. Two further TcSNMPs are expressed in supporting cells: TCSNMP1c and TcSNMP1b. But double stainings against transcripts of TcSNMP1d and TcSNMP1b never showed a localization in close vicinity. In further FISH experiments it has to be clarified whether TcSNMP1c expressing supporting cells are located next to the remaining TCSNMP1d expressing neurons. This will clarify whether it is also true for T. castaneum antenna that SNMP expressing neurons are always connected to supporting cells that also express an SNMP.

\subsection{The functional role of neuronal $T$. castaneum SNMPs in odor detection}

One prominent aim of this thesis was the analysis of the role of TcSNMPs in odor detection in T. castaneum. Given that two different TcSNMP1 homologs, namely TcSNMP1a and TcSNMP1d, are expressed in their own, individual set of neurons (figure 4.13), the hypothesis was prompted that neuronal TcSNMPs are necessary for the detection of distinct odor types. 


\subsubsection{The functional role of neuronal $T$. castaneum SNMPs in pheromone detection}

In D. melanogaster, the neuronal expressed DmSNMP1 has been shown to be indispensable for the detection of the fly pheromone cis-vaccenyl acetate (cVA; Benton et al., 2007; Jin et al., 2008). Remarkably, heterologous expression of pheromone receptors of the moth species Bombyx mori and Heliothis virescens in OSNs of D. melanogaster showed the same necessity of DmSNMP1 for the detection of the cognate pheromone (Benton et al., 2007; Li et al., 2014). Here, the tested moth and D. melanogaster pheromones, e.g. cVA or bombykol, had one similar structural feature: they contain a fatty-acid derived hydrocarbon chain (Benton et al., 2007; Regnier and Law, 1968). From these findings and due to their resemblance to the CD36 protein family, it had been hypothesized that the neuronal DmSNMP1 may mediate the detection of pheromones that have such a fatty acid chain (Benton et al., 2007; Pregitzer et al., 2014).

To test whether the identified neuronal TcSNMPs, TcSNMP1a and TcSNMP1d, are also involved in pheromone detection, their transcripts were disrupted by RNA interference, a potent method in T. castaneum, that lead to a strong and systemic response (Dönitz et al., 2014). The RNAi effect was investigated by electroantennography (EAG) (performed by Karthi Balakrishnan, Forest Zoology and Forest Conservation, University Göttingen). EAGs have been applied in several insect species, including Diptera, Lepidoptera as well as Coleoptera (Alcorta, 1991; Kurtovic et al., 2007; Nagai, 1981; Roelofs et al., 1971; Subaharan et al., 2013; 2013; Zhang et al., 2009). This technique is different to the method that was performed to study the DmSNMP1 function by Benton and colleagues (2007). These functional analyses in D. melanogaster were conducted using single sensillum recordings (SSR). This method allows to measure the neuronal activity of all OSNs housed in one single olfactory sensillum (Pellegrino et al., 2010). Unfortunately, due to missing functional analyses of single OSNs or ORs in $T$. castaneum, it was not possible to use SSR to investigate the RNAi effect. Therefore, alternative approaches, such as the mentioned EAGs were performed in this study. Additionally, the behavior of RNAi treated beetles was analyzed (not shown). To this end, an adapted circular arena was constructed and used as described in Duehl 
Discussion

et al. (2011). Here, an attractive odor of interest and a control odor (silicon oil) were added to two specific sites in the arena and it was analyzed whether the loss of neuronal TcSNMPs leads to reduced time durations spend in close proximity to the attractive odor. However, preliminary behavioral tests with different arrangements and odors (not shown), did not provide reliable data sets. No tested odor, including the aggregation pheromone 4-8-dimethyldecanal (DMD), led to a significant change of the walking behavior of the tested beetles. Even wild type beetles did not show the described attraction to the aggregation pheromone (Obeng-Ofori, 1990). This suggests a general problem of the used setup. Therefore, the RNAi treated beetles were tested by EAGs.

Knockdown of TcSNMP1a and TCSNMP1d transcripts, respectively, followed by EAG showed severe impairment in the detection of the aggregation pheromone DMD in female T. castaneum (figure 4.14). This goes well with the proposed model that SNMPs are important for pheromone detection, in particular for pheromones with a fatty acid chain (Benton et al., 2007).

Remarkably, a similar impairment was also detected in measurements with the beetle odor 1-4-benzoquinone and other pheromone like substances that have no fatty acid chain (figures 4.14 and 4.16). This is contrary to the general assumption that it is the fatty-acid derived hydrocarbon chain in pheromones that require a functional SNMP (Benton et al., 2007). This leads to the hypothesis, that SNMPs can also mediate the detection of substances without this structural feature. This hypothesis is reinforced by the identification of several antennal SNMPs in two tree killing bark beetles, Ips typographus and Dendroctonus ponderosae (Andersson et al., 2013). These beetles do use pheromones that have no long fatty acid chain, e.g. cis-verbenol (Schlyter et al., 1987). It would be interesting to know, whether the tree killing bark beetle SNMPs are involved in the detection of their cognate pheromones. Until now, no functional analyses of these SNMPs have been described. Given that the cis-verbenol detecting neurons have been characterized by SSR in I. typographus (Andersson et al., 2009), continuative SSR of snmp mutant I. typographus could test the hypothesis that SNMPs are involved in the detection of pheromones without a fatty acid chain. 


\subsubsection{T. castaneum SNMPs are involved in non pheromone odor detection}

The involvement of TcSNMPs in the detection of fatty, pheromone-like compounds without a fatty-acid derived hydrocarbon chain suggests that SNMPs are also able to mediate the detection of other fatty non-pheromone odors. This hypothesis was supported by several observations. First, SNMPs have been identified in insect species that do not even have a described pheromone system, e.g. the parasitoid beetle Dastarcus helophoroides or the malaria mosquito Anopheles gambiae (Vogt et al., 2009; Wang et al., 2014). Furthermore, in D. melanogaster it has been shown, that the neuronal DmSNMP1 is required for a proper response to the non pheromonal fatty odor farnesol (Ronderos et al., 2014). This odor has no simple fatty acid chain and is a component of ripe citrus peels, a potential egg laying substrate of D. melanogaster (Ronderos et al., 2014; Rowat et al., 2005).

The results of this study, showing that neuronal TcSNMPS of T. castaneum are involved in the detection of fatty food odors such as wheat germ oil and ß-ionone (figure 4.15), reinforce this hypothesis further. Wheat germ oil is a typical component of the preferred ecological habitat of T. castaneum: older and damaged grain with high fatty acid content. In behavioral analysis, it has been shown that $T$. castaneum is attracted by various grain oils, including wheat germ oil (WGO) (Phillips et al., 1993).

Taken together, this leads to the suggestion that different insects use SNMPs to detect fatty volatile chemicals emitted from sources that are crucial for their respective life requirement. These fatty volatile odorants can include different type of pheromones, food odorants or potential egg laying sites.

\subsubsection{TcSNMP1a might be more important for female beetles}

Knockdown of TCSNMP1a showed a differential effect in male and female $T$. castaneum. Except for few tested food odor concentrations (figure 4.15), the TCSNMP1a knockdown did not influence the neuronal responses toward the tested odorants in male T. castaneum. In females, however, TcSNMP1a knockdown led to slightly reduced responses to all tested odors. 
Discussion

This sex specific difference of TCSNMP1a knockdown beetles indicates that the tested odors are more important for female beetles and that TCSNMP1a is needed for an accurate detection of these substances. A possible explanation for that finding is that TcSNMP1a might be needed for the detection of volatiles emitted by substances that are more important for females, such as suitable egg laying sites. This hypothesis could be tested by behavioral tests of female beetles that were treated with dsRNA targeting TcSNMP1a-transcripts. Here, it would be interesting to analyze, whether RNAi treated beetles would find appropriate egg laying sites and consequently would lay the same amount of eggs in this area as control females.

\subsubsection{Outlook}

The loss-of-function experiments conducted in this study extended the selection of potential odors that need a functional neuronal TcSNMP. Continuative experiments are needed to investigate the role of TcSNMPs in T. castaneum in more detail. First, experimental replicates should be done with non-overlapping dsRNA fragments to exclude off target effects. Furthermore, a broader set of odorants has to be tested, also non fatty odors, like alcohols or fruit esters. In D. melanogaster, it has been shown for several fruit esters that they are detected independently of the DmSNMP1. Therefore it would be interesting to analyze, whether this is also true for TcSNMPs.

\subsection{T. castaneum SNMP1 diversification might mirror specialized coleopteran ecologic adaptation}

The function of the neuronal DmSNMP1 homolog of D. melanogaster is highly conserved between Diptera (D. melanogaster) and Lepidoptera (B. mori, $H$. virescens, A. polyphemus), despite an evolutional divergence of 290 million years (Benton et al., 2007; Kurtovic et al., 2007; Li et al., 2014). This conserved function has been shown by functional analyses of heterologously expressed moth 
pheromone receptors in D. melanogaster OSNs. These pheromone receptors were able to detect their cognate pheromone in dependence of a functional DmSNMP1 (Benton et al., 2007; Kurtovic et al., 2007; Li et al., 2014).

In contrast to that, heterologously expressed SNMP homologs from the coleopteran T. castaneum in pheromone sensitive OSNs of D. melanogaster were not able to mediate the detection of the fly pheromone cVA (figure 4.19). This indicates a divergent specialization of the beetle's and fly's SNMP1 function. The order Coleoptera diverged over 10 million years earlier within the holometabolous lineage than Diptera and Lepidoptera (Grimaldi and Engel, 2005). Hereby they became the most diverse insect order occupying many different ecological niches (Maddison et al., 2007). The potential for fast evolvement of existing genes might have helped to colonize new environments.

For ORs and OBPs gene duplication is believed to be a characteristic mechanism to adapt to new environments (Gardiner et al., 2008; Hansson and Stensmyr, 2011; Wang, 2009). Therefore one could presume, that SNMPs as further odor detection protein, have to coevolve with the OR/OBP protein family by similar mechanisms. Interestingly, the chromosomal localization of TcSNMP1a, TcSNMP1b, TcSNMP1c and TCSNMP1d suggests that these four genes are the result of gene duplication events (Vogt et al., 2009). This implies that the original set of SNMP genes (one SNMP1 homolog and one SNMP2 homolog) of a common ancestor underwent evolutionary radiation and enabled the adaptation to various and novel ecological situations.

This hypothesis is supported by the finding that the identity of amino acid sequences of the different TcSNMP1 homologs is low, which suggests fast molecular evolution and functional derivation. In contrast, SNMP1 homologs of different moth species show higher amino acid conservation even across species (Forstner et al., 2008). This indicates that after gene duplication events further alterations of the gene sequence were needed to become perfectly adapted to the occupied ecological niche. 


\subsection{TcSNMPs have conserved subdomains in the extracellular loop}

By prediction of the protein topology of the obtained putative amino acid sequences two transmembrane domains (TMDs) were identified (figure 4.2). These TMDs were flanked by short intracellular N- and C-termini. Between the two TMDs one big extracellular loop was predicted. Sequence analysis of SNMP proteins of other insects have been shown to have a similar protein topology (Nichols and Vogt, 2008). Because of this protein structure and further sequence similarities, SNMPs have been grouped to the big CD36 protein family (Rogers et al. 2001). For several members of this protein family it has been shown that they interact with fatty acids, lipids or lipid protein complexes (Febbraio and Silverstein, 2007; Lai et al., 2013; Sakudoh et al., 2013). Furthermore, studies about the human CD36 protein scavenger receptor class B type I (SR-BI) showed that subdomains of the extracellular loop are responsible for the binding of a given ligand (Kartz et al., 2014). Interestingly, although the TcSNMPs showed generally low identity values among each other (figure 4.3), within the putative, about 400 bp long extracellular loops, 31 scattered amino acids were identical between all TcSNMPs (figure 4.2). Moreover, 26 of these amino acids are also shared with the SNMP1 and SNMP2 homologs of D. melanogaster (not shown). The functional analysis of this study showed that the TcSNMPs have a different function compared to the DmSNMPs (chapter 4.4.1, figure 4.19). Therefore, the 26 identical amino acids shared by TcSNMPs and DmSNMPs are most likely important for structural purposes. The five amino acids that are identical between all $T$. castaneum SNMPs, but differ from D. melanogaster SNMPs are located between the amino acid aspartic acid at alignment position 151 and the proline at alignment position 332 (figure 4.2). To test whether these five amino acids are involved in the mediation of a given odorant, one could exchange this area within a neuronal TcSNMP (TcSNMP1a or TcSNMP1d) with the equivalent area of the DmSNMP1 by the CRISPR/Cas9 technology (Gilles and Averof, 2014). Subsequently, one could analyze the functionality of the chimeric TcSNMPs by electroantennography. 


\subsection{DmSNMP2 is able to compensate for loss of DmSNMP1 function in cVA detection in the antenna}

DmSNMP2 is prominently expressed in the body of D. melanogaster and has no described role for olfaction (Chintapalli et al., 2007). Surprisingly, heterologously expressed DmSNMP2 isoform B was able to facilitate the detection of the fly pheromone cVA in pheromone sensitive OSNs of transheterozygous snmp1 mutant flies (figure 4.19). In contrast to that, studies done in human embryonic kidney cells expressing the $H$. virescens pheromone receptor with or without $H$. virescens SNMPs (HvSNMPs), showed that HvSNMP2, in contrast to HvSNMP1, does not influence the detection of the moth pheromone (Z)-11-hexadecenal (Pregitzer et al., 2014). Even though HvSNMP2 is expressed in supporting cells in the moth antenna and is part of the pheromone detection system (Forstner et al., 2008), HvSNMP2 function in olfaction appears to be more specialized compared to the HvSNMP1 that is expressed in the pheromone detecting neurons (Forstner et al., 2008). This situation is entirely different to D. melanogaster, where DmSNMP2 does not have a described role in olfaction, but can fulfill similar functions like the DmSNMP1 (figure 4.19) that is essential for pheromone detection (Benton et al., 2007).

Interestingly, the DmSNMP2 isoform $\mathrm{C}$ was not able to mediate the detection of cVA (figure 4.19). Compared to DmSNMP2 isoform B, isoform C has one additional exon that leads to 46 additional amino acids (Hoskins et al., 2007). From my findings, it appears that the absence of these additional amino acids allowed cVA detection. This might be due to different protein folding. Alternatively, removal of this additional stretch may connect two parts of the protein to a functional area. Protein predictions indicated that in this area an alpha-helix is formed and that the connected parts are located in this helix (figure 4.22).

Therefore it might be possible that this helix is responsible for the interaction and mediation of cVA. In order to characterize the functional area of DmSNMPs in more detail it would be interesting to focus on the region containing this predicted helix. To test whether this helix is sufficient to enable cVA mediation one would replace this helix in the DmSNMP1 protein with the helices of DmSNMP2 isoform B and DmSNMP2 isoform C (figure 5.2). Subsequently, one would test the rescue abilities 


\section{Discussion}

of these chimeric SNMPs by using the rescue assay system. In case that the DmSNMP2C helix in the chimeric DmSNMP1 protein would abolish the capacity to detect cVA, this would indicate that the endogenous DmSNMP1 helix is necessary for cVA mediation. In case that this chimeric DmSNMP1 would still be able to mediate cVA detection this would indicate that other regions of the DmSNMP1 protein are still sufficient for cVA detection, and therefore involved in this process. Similarly, if the cVA mediation in a rescue experiment using a chimeric DmSNMP1 protein containing the DmSNMP2B helix, would be more effective than in the rescue with the native DmSNMP2B protein, detectable by a higher neuronal calcium response, this would also indicate that other parts of the DmSNMP1 protein are involved in the cVA mediation.

Another approach would be to analyze this particular helix of the DmSNMP1 protein by using other SNMPs as scaffold for the DmSNMP1-helix. Here, one would exchange the corresponding helices of the DmSNMP2B and the TcSNMP1a with the DmSNMP1 helix (figure 5.2). Analogously to the other chimeric proteins, subsequent functional analysis using the rescue assay system would clarify, whether this DmSNMP1 helix is sufficient to mediate cVA detection. If the DmSNMP1 helix in the TcSNMP1a protein would be able to mediate cVA detection, this would suggest that the DmSNMP1 helix is sufficient for a proper DmSNMP1 function. Analogously, if the DmSNMP2B protein containing the helix of the DmSNMP1 protein instead of its own helix would enhance the neuronal response compared to the native DmSNMP2B protein, this would also indicate that the DmSNMP1 helix contains the functional region of DmSNMP1.

Furthermore fusion of the DmSNMP1 protein with the DmSNMP2C protein within this helix would allow to test whether the $\mathrm{N}$ - or C-terminal region of the helix or the protein is equally involved in the function of DmSNMPs (figure 5.2). Depending on the results, chimeric proteins would subsequently be designed that contain increasing amino acid stretches of the native DmSNMP1. This would allow the identification of the functional area of the DmSNMP1 and therefore give the opportunity for further experiments that will help to clarify the exact molecular mechanism of DmSNMPs. 


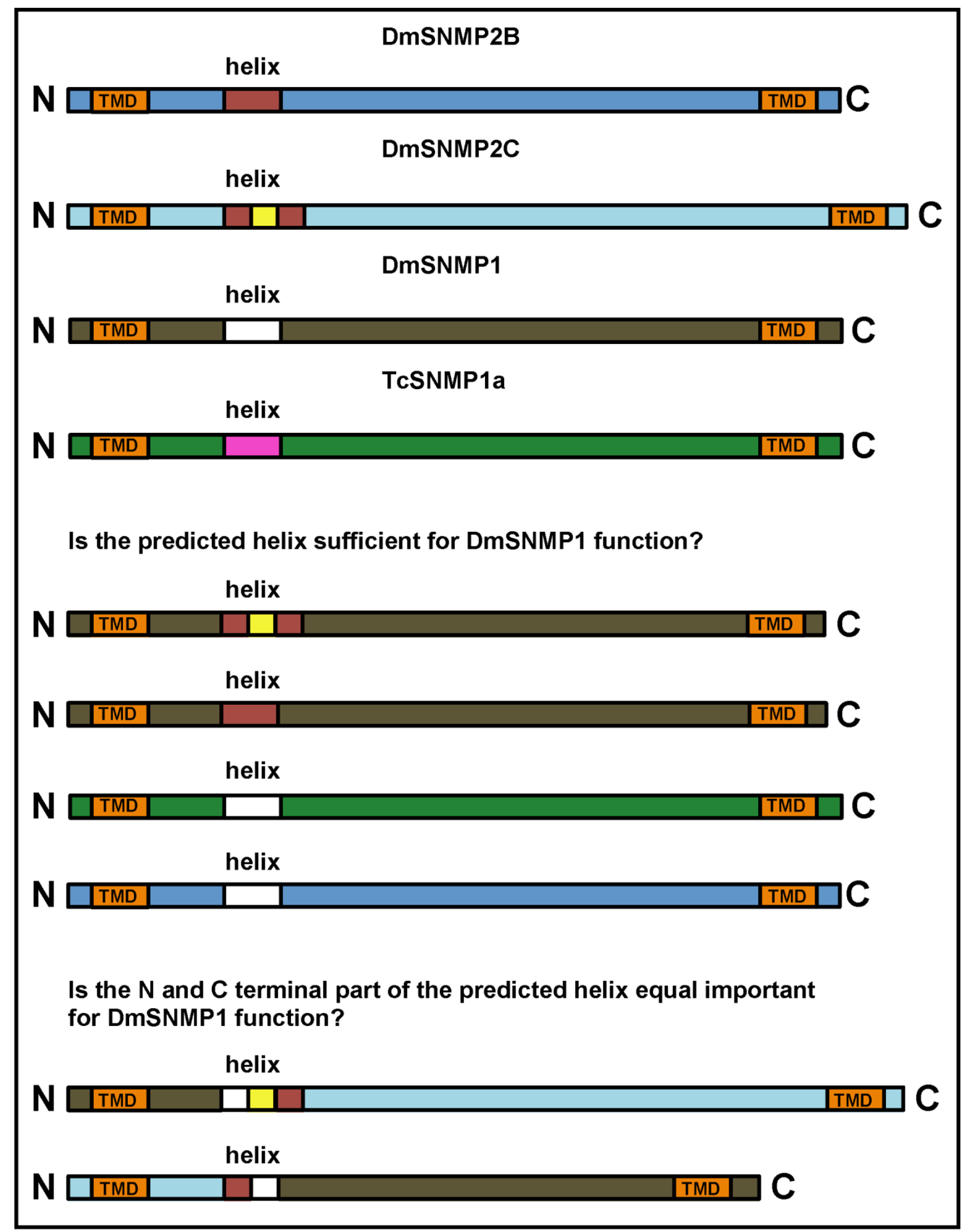

Figure 5.2 Potential chimeric constructs for further analysis

Shown are schematic representations of different SNMPs as indicated and potential chimeric proteins. DmSNMP2 isoforms are shown in blue, DmSNMP1 is shown in grey, and TcSNMP1a is shown in green. Important predicted protein domains are shown in boxes as indicated. Helix boxes of the same color indicate same sequence. Abbreviations: N: N-terminus, C: C-terminus, TMD: transmembrane domaine, helix: predicted alpha helix of interest. 


\subsection{T. castaneum SNMP2 influences the odor response profile of the $D$. melanogaster pheromone receptor 0R67d in snmp1 mutant flies}

In this work, the D. melanogaster DA1 glomerulus was used for in vivo calcium imaging measurements. Neurons that form the DA1 glomerulus express the cVA sensitive receptor 0R67d (van der Goes van Naters and Carlson, 2007; Ha and Smith, 2006; Kurtovic et al., 2007). As discussed below, neurons expressing this odorant receptor normally do not respond to 1-hexanol (Vosshall et al., 1999). In contrast, they are even inhibited by 1-hexanol (figure 4.20). Surprisingly, when heterologously expressed in snmp1 mutant D. melanogaster, TcSNMP2 mediated the detection of 1-hexanol in these neurons. This indicates that TcSNMP2 changes the response profile of this highly specific pheromone receptor, that is narrowly tuned to cVA (Kurtovic et al., 2007). A recent study hypothesized that DmSNMP1 is necessary for the binding of cVA to the $0 R 67 d$ as well as for its removal from this receptor (Li et al., 2014). Based on this model, the hypothesis states that snmp1 mutant flies are no longer able to detect cVA after the 0R67d is blocked by bound cVA (Li et al., 2014). This hypothesis was confirmed by SSRs of snmp1 mutant OR67d OSNs of female flies that had never smelled cVA before (cVA-virgins), because these flies were able to detect the first cVA application of their life (Li et al., 2014).

From this hypothesis and from the findings in this work, regarding 1-hexanol detection in snmp1 mutant flies that express TcSNMP2, the following hypothesis could be assumed: concerted action of TcSNMP2 and the solvent 1-hexanol might be able to remove bound cVA, thus mimicking endogenous DmSNMP1 function. Afterwards, the dissociated cVA would be able to bind again to the unblocked OR67d and, hence, be detected anew. This proposed mechanism would also explain the delayed and rather weak response elicited by 1-hexanol (figure 4.20). To test this model, a first set of measurements of cVA-virgins were conducted. According to the proposed mechanism, cVA-virgins would not show a response to 1-hexanol, since the observed 1-hexanol response would be restricted to flies that have already smelled cVA at least once. In case that the cVA virgins were able to detect 1-hexanol, this would suggest that TcSNMP2 indeed changes the response profile of the pheromone receptor 0R67d. In case they don't, this would indicate a 
cleaning function of 1-hexanol and a partial ability of TcSNMP2 to substitute for DmSNMP1 in an snmp1 mutant background. No 1-hexanol response was observed in these flies. However, the survival rate of them was very low, indicating a general problem of the physiology and vitality of the tested flies. Therefore, this experiment has to be repeated.

\subsection{T. castaneum SNMP1a and SNMP1b.1 influence presynaptic inhibition within the antennal lobe of snmp1 mutant D. melanogaster}

Generally, pheromone sensitive neurons are inhibited by most other odors (Agarwal and Isacoff, 2011; Pregitzer et al., 2012). In this study this observation was confirmed by calcium imaging measurements of the glomerulus DA1, the first relay station of the cVA reactive neurons (Datta et al., 2008), after application of 1hexanol and isoamyl acetate, respectively (figures 4.20 and 4.21). Presynaptic inhibition of olfactory sensory neurons has been described to be mediated by inhibitory local interneurons (LNs) (Ignell et al., 2009). The majority of these LNs release gamma-aminobutyric acid (GABA) (Mikael A Carlsson, 2010). Many other substances have been described to influence the presynaptic inhibition as well, such as different neuropeptides, glutamate or serotonine (Dacks et al., 2009; Ignell et al., 2009; Mikael A Carlsson, 2010; Olsen and Wilson, 2008; Root et al., 2008; Wilson, 2013). Interestingly, this presynaptic inhibition was not detectable in D. melanogaster snmp1 mutant DA1 neurons that heterologously expressed TcSNMP1a or TcSNMP1b.1 (figures 4.20 and 4.21). This indicates an extended function of SNMPs apart from the mediation of odorants. It suggests that TcSNMP1a and TcSNM1b.1 are able to modify the inhibitory antennal lobe circuitry, leading to missing inhibition of DA1 neurons.

The observation that the isoform TcSNMP1b.2, that has an 84 amino acids shorter putative extracellular loop, did not show this effect indicates that the area that is responsible for the reduced presynaptic inhibition is located in this loop (figures 4.20 and 4.21). Therefore one could assume that TcSNMP1b.1 has a special functional structure within its extracellular loop. This might allow the binding and capturing of one or more of the several described inhibitory components, such as 
Discussion

GABA and thereby thinning the amount of inhibitory components. However, generally, these inhibitory components are no fatty acid derivatives and therefore no classical target for SNMPs. This argues against the described titration effect.

Because SNMPs are members of the CD36 protein family, further molecular mechanisms are possible that would allow TcSNMP1a and TcSNMP1b.1 to modulate the presynaptic inhibition. Recently, it has been shown that human CD36 mediates the recognition and hetero-dimerization of different Toll-like receptors (Stewart et al., 2010). Analogously, it could be possible that TcSNMP1a and TcSNMP1b.1 influence and modify the response of receptors that interact with inhibitory components, such as the GABA receptors (GABA-R) (Wilson and Laurent, 2005). One precondition for this hypothesis is that TcSNMPs are located in proximity to GABA-Rs. Immunohistochemical approaches with antibodies against GABA-R and TcSNMP1a or TcSNMP1b.1 would clarify whether this condition is fulfilled.

To discriminate between both hypotheses, one would express e.g. GABA-R with or without TcSNMP1a or TcSNMP1b.1 in D. melanogaster cell lines, such as Schneider 2 cells (Schneider, 1972). Here, one would test whether the GABA induced GABA-R response is modified in the presence of TcSNMP1a or TcSNMP1b.1. If there is a change in response, one would subsequently analyze the different hypotheses for their correctness. If the first hypothesis that TcSNMP1b.1 capture specific inhibitory components (in this case GABA) would be correct, this effect would be titratable by addition of more GABA. If not, this would hint to the second hypothesis, that TcSNMP1a and TcSNMP1b.1 modify the GABA-R. Subsequently, one would study the mechanisms behind that modification. Following this approach, one could also analyze other inhibitory components and their receptors like glutamate/glutamate receptor or short neuropeptide F (sNPF)/sNPF receptor (Liu and Wilson, 2013; Mertens et al., 2002). This will allow to understand how SNMPs are able to influence the presynaptic inhibition, a capability that extends the known function and importance of SNMPs. 


\subsection{Novel roles of SNMPs}

The different functional analyses of the numerous TcSNMPs made it possible to extend the knowledge of the functional capacities of these proteins. For a long time the role of SNMPs was believed to be restricted to the mediation of pheromones (Benton et al., 2007; Jin et al., 2008; Li et al., 2014). The heterologous expression of TcSNMPs in D. melanogaster showed that SNMPs might have additional molecular functions in addition to the mediation of odors. They appear to be able to reduce presynaptic inhibition of a given OSNs. Thereby they could support the detection of any given odor. The functional analysis of TcSNMPs in T. castaneum hinted to the idea that SNMPs help to detect odors that are emitted by sources that are crucial for the specific life requirements of an insect. Recent studies in D. melanogaster support this suggestion. It has been shown that the maximal firing rate was decreased in farnesol detecting neurons without a functional DmSNMP1 (Ronderos et al., 2014). Farnesol is a volatile component of citrus fruit peels, a substrate that female flies prefer as egg laying site (Dweck et al., 2013). Taken together I propose the following functions for SNMPs: SNMPs are indispensable for the mediation and removal of pheromones. Without an appropriate neuronal SNMP1, pheromones cannot be detected anymore (Benton et al., 2007; Jin et al., 2008; Li et al., 2014; this study). Furthermore, SNMPs intensify the neuronal responses towards specific odors that play an fundamental ecological role for a given insect (Ronderos et al., 2014, this study). Depending on the occupied ecological niche this odors can be different, which might explain the varying number and high level of divergence of identified SNMPs in different insects. Due to the extended importance of SNMPs it will be crucial to identify further SNMP mediated odorants and to clarify the molecular mechanisms of SNMP function in more detail. 


\section{Supplemental}

\subsection{Full-length cDNA sequences and amino acid sequences of TcSNMPS}

\subsubsection{TcSNMP1a}

\section{cDNA sequence}

>AGTGAATGAAACCGATTCGTCGGAAGGACTTCTTATTTTTACTTTTTCCTAAGTTGCGTTTACTTAAGT TCTCTTTTGATTCGTTAAAGTGCATTTATCATTGTTACACGAAGAAGAAAACTAAAAAGGAAATGCGGCT GCCGGTCAAAATCGCGATCGGATGCACGATTGGGCTCGTTGTTATCATTGTTTTCGGATTTATTGCTTTT CCTAAAATGATCAAAGGCAAAGTGAAGAGTATGATAAATTTGAACAAGGGGAGCGAAATCCGCCAAATGT TCGTCAAAGTCCCGTTCGCTCTAGACTTTAAAATTTATATGTTTAACGTAGCAAATCCGATGGATGTCCA GAAGGGTGCTTTGCCTGTCCTAAAAGAAGTGGGACCGTTCTGTTTCGAAGAATGGAAGGAGAAGGTTGAC CTTGACGATAACGACGACGAGGATGTCATGTTTTACAATCCCAAGGACACGTTTTACAAAACTAACGGGC CTGGATGCCTCGACGGAAGCCAGATGATTACAATGGCACATCCTTTAATACTCGGGATGGTGAACACAGT GGTGCGGACTAAACCAGGCGCCATTTCCTTGATTAGCAAAGCCATTAATTCCATTTACGGCAATCCTGAT TCTATTTTTATGACTGCTTCGGCTATGGACATCCTGTTTGATGGTGTTGTAATTAAATGCGGGGTCAAAG ATTTCGCCGGAAAGGCCGTTTGTTCGCAGCTGAAAGAAGCACCAGACTTGAGGCATGTCGACGAAAATGA TTTGGCGTTTTCATTTATAGGGCCTAAAAATGCAACTCCCGGGAAGAGATTTAAAGTTCTGAGGGGCGTC AAGAAATCGCACGATGTGGGACGGATTCTCGAGTATGACAATAAGAAAGAAATGGAAGTGTGGCCTACGA AAGAATGTAACCAATATAAAGGAACAGACGGGACGGTATTTCCCCCTTATCTCACAAAGGAGGAAGGTCT TGCCTCCTATGCGCCCGACTTGTGCCGTTCGTTAGTTGCCGTCTACAGTGGAGACACAAAATACGACGGT ATTCCTGTGCGAATATACACGGCGACTTTAGGCGACATGTCCAAAAATGCAGACGAGAAGTGTTACTGCC CCACACCTGACACTTGCCTGAAGAAGGGCATGATGGACCTGTTCAAGTGTGCAGGAGTTCCTGTCTATGT CTCACTACCACATTTCTACGAATCGGACGAAAGCTACGTCAAAGGAGTCGTCGGCCTTAACCCTAACAAG AAAGACCACGGAATTCAGATTTTGTTCGAATCGACAACTGGGGGTCCTGTCAAAGCCGCGAAACGCCTCC AGTTCAACATGCCCCTGGAGCCGAATCCAAAATTGCCAATTTTTGCAAACTTGCCCAACACAGTTTTGCC ACTGTTCTGGGTGGAGGAAGGCGTGGCGCTGAATAATACGTTTACGAAACCGTTGAAAGACCTTTTCAAA ATAATGAAGATTGTGAAGATCGCGAAATGGTTGATTATGCTGGGATGTTTGGGGGGTTTGGGAGCCGCCG GTTATCTCTACTTTTCCAAAAAAGGGGAAGCGAATATTACGCCGGTACATAAGGTTAAACCGGCCGAAAA TGGGGTTAGCACTCTCGGGGGTGAAGTCAACCATGCCATGTCTGATAACGAGATAGAGAAATACTGATGA TTTGTTTAGGCAGGTTCTGTTCTAGTCTTGTTGGAAGATTGCTAGTTTATTTTTATATATTTTTGTATTA AAGCGGTGTGTTTAGTTAACGATTAGTTAATTATTTGTGCACGATTTTTTAACTGTTTTAGTCTGTGATT TAGTGATTATGTATGAGATATGGTTAGTTTATTTAACTTTAGAAAAATGTTATATTGACAGGGTATGCTA GTCAGAAAAATAAATATATTATTTGTAATTTGCTTTTATTTCAAATACACTTTATCTTGAAAAAAAAA AAAAAAAAAAAAAAAAAA GT

\section{Amino acid sequence}

>MKPIRRKDFLFLLFPKLRLLKFSFDSLKCIYHCYTKKKTKKEMRLPVKIAIGCTIGLVVIIVFGFIAFP KMIKGKVKSMINLNKGSEIRQMFVKVPFALDFKIYMFNVANPMDVQKGALPVLKEVGPFCFEEWKEKVDL DDNDDEDVMFYNPKDTFYKTNGPGCLDGSQMITMAHPLILGMVNTVVRTKPGAISLISKAINS IYGNPDS IFMTASAMDI LFDGVVIKCGVKDFAGKAVCSQLKEAPDLRHVDENDLAFSFIGPKNATPGKRFKVLRGVK KSHDVGRI LEYDNKKEMEVWPTKECNQYKGTDGTVFPPYLTKEEGLASYAPDLCRSLVAVYSGDTKYDGI PVRIYTATLGDMSKNADEKCYCPTPDTCLKKGMMDLFKCAGVPVYVSLPHFYESDESYVKGVVGLNPNKK DHGIQILFESTTGGPVKAAKRLQFNMPLEPNPKLPI FANLPNTVLPLFWVEEGVALNNTFTKPLKDLFKI MKIVKIAKWLIMLGCLGGLGAAGYLYFSKKGEANITPVHKVKPAENGVSTLGGEVNHAMSDNEIEKY* 


\subsubsection{TcSNMP1b.1}

\section{cDNA sequence}

>AGTAAGCTTGCAACTTAGTATTGTGAAGTTATTTTAAAACATGACCACTTGCTTTAACACAAAATTAAA ATCGTTTCATGTAATTTTGATGTCTCCACAATAACTCTCGTTAATACCCTATTACTCGGCTTCATATTT TACATGCCATGTCTAAGGTCTATGGTCAAATGGCAAAGACAGCTAAAACCGGGAAACGAAGTGCGCGATT TCTACATCAAACTACCAATTCCTTTAGATTTCCGAGTCTATTTTTTTAACATTTCTAATCCGGAGGAAGT AAAACAAGGCGAAAAACCCATTCTGAAACAGATTGGTCCGTACTGTTACGATGCCTACAAGGAAAAAATC AACGTCGAAGACGACAAAGACAATGACACTCTCACTTACAACCCTTACGACACTTATTTTTTTAACCAAA TGAGAACGGGTGATTTATCACAAGATGATTACGTTACAATTCTTCACCCCCTCACTGTGGGGATTGTAAA CGCAGTCGCCACCCAAAAACCCCAATACCTCTCAGCGGTAAATAAGGCCCTCCCTGTCATTTTCAAAGAA AACAGCTCGATTTACCTCACTGCAAAAGTGCGCGAGATACTTTTCGACGGCATTTTGATAAATTGCAACG TTAAAGACTTTTCGGCAAATGCTGTGTGTTCACAATTCAAGGGCCAACCGGCCATGGTGGAAGTCGAAAA AAATATTTACAGTTTTTCCCTACTAGGCTCGCGAAATGGTTCCATCCCCACACGTATCACCATACACAGA GGGGTTAAAAACGCAGCTGATATCGGCCGTGTGGTCACAATTGATAACAAAACAGACCTGGATGTGTGGC CTGAACCAGAATGTAACGCCTTTCGGGGAACTGACGGTTGGGTTTTTCCCTCATTTTTGGAAAAGGAAGA CGGGATCTGGACCGTAGCCTCGGATTTGTGCAGAAGTTTCAAAGCTCAGTACGTGGAAGATTTGAAGTTT CACGGTGTCGTTGTCCGAAAATACTTTGCCGATCTTGGTGACATGTCATCAAACCCTGCCGAGAAATGTT TCTGTCCAGCACCCGAAAAGTGCCTACCTAAGGGCGTAATGGACTTGACCAAGTGCATGAAAGTGCCTTT ATACTGCACTTTGCCCCATTTTCTGAGAGCCGATGAAAAATTATTGCAACAAGTCGAGGGTCTCAGTCCT GAGCTTGAAAGACACATTATAAAAATCTACTTTGAGCCTTTGACCGGAACTCCAATGTTGGGTCAAAGAC GAATTCAGTTCAATTTGCAACTAATGCCAATTCCCAAAGTTGCGATGATGAAAACGGTACCTGAAGCCCT CCATCCGATTTTGTGGATCGAAGAAGGAGTCGAACTTGAAGGGTTTCTTTTGAAAAAAGTGACCTCAGTG TTTACATTGCTTAAACTAATGACTTTTATTCGGTACATAATGTTGGGGCTTTCCATCCAGGGAATATTAT ATGGAGGCTACAAGCTGTATCAAGAGTCCAAAAGTAAGAAAGTTAGTCCGGTGCAAAATGTGACGACGGC ATCCAAAAACCAAAATCAAGGAAAAACTGGTGGCATTGAACTACCATCGATGAATAAGAGAAATAAAGAA AATACCAAAAATGCTAATATTGTAGTTTGGACAATTATGTATTTTTGAGAATACTTGTAATAAACGA TTTTTAC

\section{$\underline{\text { Amino acid sequence }}$}

>MTTCFNTKLKSFHVILIVSTITLVNTLLLGF I FYMPCLRSMVKWQRQLKPGNEVRDFYIKLPIPLDFRV YFFNISNPEEVKQGEKPILKQIGPYCYDAYKEKINVEDDKDNDTLTYNPYDTYFFNQMRTGDLSQDDYVT ILHPLTVGIVNAVATQKPQYLSAVNKALPVIFKENSS IYLTAKVREI LFDGILINCNVKDFSANAVCSQF KGQPAMVEVEKNIYSESLLGSRNGS I PTRITIHRGVKNAAD I GRVVT I DNKTDLDVWPEPECNAFRGTDG WVFPSFLEKEDGIWTVASDLCRSFKAQYVEDLKFHGVVVRKYFADLGDMS SNPAEKCFCPAPEKCLPKGV MDLTKCMKVPLYCTLPHFLRADEKLLQQVEGLSPELERHIIKIYFEPLTGTPMLGQRRIQFNLQLMPIPK VAMMKTVPEALHP ILWIEEGVELEGFLLKKVTSVFTLLKLMTF IRYIMLGLS IQG I LYGGYKLYQESKSK KVSPVQNVTTASKNQNQGKTGGIELPSMNKRNKENTKNA* 


\title{
Supplemental
}

\subsubsection{TcSNMP1b.2}

\section{cDNA sequence}

>AGTAAGCTTGCAACTTAGTATTGTGAAGTTATTTTAAAACATGACCACTTGCTTTAACACAAAATTAAA ATCGTTTCATGTAATTTGATTGTCTCCACAATAACTCTCGTTAATACCCTATTACTCGGCTTCATATTT TACATGCCATGTCTAAGGTCTATGGTCAAATGGCAAAGACAGCTAAAACCGGGAAACGAAGTGCGCGATT TCTACATCAAACTACCAATTCCTTTAGATTTCCGAGTCTATTTTTTTAACATTTCTAATCCGGAGGAAGT AAAACAAGGCGAAAAACCCATTCTGAAACAGATTGGTCCGTACTGTTACGATGCCTACAAGGAAAAAATC AACGTCGAAGACGACAAAGACAATGACACTCTCACTTACAACCCTTACGACACTTATTTTTTTAACCAAA TGAGAACGGGTGATTTATCACAAGATGATTACGTTACAATTCTTCACCCCCTCACTGTGCGAAATGGTTC CATCCCCACACGTATCACCATACACAGAGGGGTTAAAAACGCAGCTGATATCGGCCGTGTGGTCACAATT GATAACAAAACAGACCTGGATGTGTGGCCTGAACCAGAATGTAACGCCTTTCGGGGAACTGACGGTTGGG TTTTTCCCTCATTTTTGGAAAAGGAAGACGGGATCTGGACCGTAGCCTCGGATTTGTGCAGAAGTTTCAA AGCTCAGTACGTGGAAGATTTGAAGTTTCACGGTGTCGTTGTCCGAAAATACTTTGCCGATCTTGGTGAC ATGTCATCAAACCCTGCCGAGAAATGTTTCTGTCCAGCACCCGAAAAGTGCCTACCTAAGGGCGTAATGG ACTTGACCAAGTGCATGAAAGTGCCTTTATACTGCACTTTGCCCCATTTTCTGAGAGCCGATGAAAAATT ATTGCAACAAGTCGAGGGTCTCAGTCCTGAGCTTGAAAGACACATTATAAAAATCTACTTTGAGCCTTTG ACCGGAACTCCAATGTTGGGTCAAAGACGAATTCAGTTCAATTTGCAACTAATGCCAATTCCCAAAGTTG CGATGATGAAAACGGTACCTGAAGCCCTCCATCCGATTTTGTGGATCGAAGAAGGAGTCGAACTTGAAGG GTTTCTTTTGAAAAAAGTGACCTCAGTGTTTACATTGCTTAAACTAATGACTTTTATTCGGTACATAATG TTGGGGCTTTCCATCCAGGGAATATTATATGGAGGCTACAAGCTGTATCAAGAGTCCAAAAGTAAGAAAG TTAGTCCGGTGCAAAATGGGACGACGGCATCCAAAAACCAAAATCAAGGAAAAACTGGTGGCATTGAACT ACCATCGATGAATAAGAGAAATAAAGAAAATACCAAAAATGCTTAATATTGTAGTTTGGCACAATTATTG TATTTTGAGAATACTTGTAATAAACGATTTTAC

\section{Amino acid sequence}

\begin{abstract}
$>$ MTTCFNTKLKSFHVILIVSTITLVNTLLLGFIFYMPCLRSMVKWQRQLKPGNEVRDFYIKLPI PLDFRV YFFNI SNPEEVKQGEKP I LKQ IGPYCYDAYKEKINVEDDKDNDTLTYNPYDTYFFNQMRTGDLSQDDYVT I LHPLTVRNGS I PTRITIHRGVKNAADIGRVVTIDNKTDLDVWPEPECNAFRGTDGWVFPSFLEKEDGIW TVASDLCRSFKAQYVEDLKFHGVVVRKYFADLGDMS SNPAEKCFCPAPEKCLPKGVMDLTKCMKVPLYCT LPHFLRADEKLLQQVEGLSPELERH I IKIYFEPLTGTPMLGQRRIQFNLQLMP I PKVAMMKTVPEALHP I LWIEEGVELEGFLLKKVTSVFTLLKLMTFIRY IMLGLS IQG I LYGGYKLYQESKSKKVS PVQNGTTASKN QNQGKTGGIELPSMNKRNKENTKNA*
\end{abstract}




\subsubsection{TcSNMP1c}

\section{cDNA sequence}

>AGTGCAATATGCTTTCTTACAAGAAGATAACTATTATATCAGCATGTTGCTTTGTTGTGACGATCATTT TTGGGTTCGTTGCTTACGAACCAATTTTTGAATATGCAATTAGAGACCAAATTTCTCTGCATCGGAGGAA CTTTGTGAGACAGTTTTACCTAAAGTACCCAATACCGTTGGATTTCCGTGTCAATTTTTTCAACGTTTCA AACCCTGACGAAGTAGAAAACGGTGGAGTCCCTGTTTTGAGTGAAGTCGGACCTTACTGCTATGATCTTT ACAAGGAGAGAATAGACGTGGAGGACAACGAGGCCGAGGACAGCCTCACCTACACCCCTTACGACATTTA СTTATTTAATCAGGAGAGATCGGGAAACTTAAGTCAGGATGATTACGTTACAATTATTCACCCCCTTGTC GTGAGCCTTGTCAATTACGTGTCAGTGAAGACGCCTCACTATTTACAGTTTCTAAACGACGCACTTGGAT TTCTGTTCCCTGAAAAATCCATCTTTCTTACCGCCAAAGTCAGAGATATTCTATTTGACGGAATGTTAAT AAACTGCACGTCTCGGGATTTCACAGCGATGGCAGTTTGTACACAAATACGAACAAAAATTCCCGGAATA CAGTTTGAAAGTAAAGATTATTTGAAATATGCGCTCCTTGGACAACAAAATGGAACTTTACCTACTAGAA TAACGGTTTTGAGAGGCATAAAAGAGTCAGAGAATTTGGGCAAATTGGTGGCTGTTGATAACGTGACAAA GAGTGATTTCTGGTCAAATGAAGAATGTAATGAGTATAAGGGCACCGATGGTTGGATTTTCCCCCCGTTT TCTGGGAGGTTGAAGACGATCTGGATGCACGCAACAACACTGTGCCAAAATATCCATGCCGATTTCGTTG GTCCTGCTACAAGTAACGGTTTTGCAGTAAATAAATACTACTCTGACTTTCAAAATATTTGTACGAATTG CTCGCTTCAAGAGCCTTGCCTTCCGGAAGGATTAATCGACGTCACGAAATGTCTAACAGCTCCTATTTAC ATCAGTTTACCCCACTTTTTGCGAAGCGATGAAAGTCTGATTCGCGGGGTCAAAGGCTTGAATCCTGATA CTGAAAGTCACATCACGAGAATTCTCCTCGAGGGGACACTGTCGCTTCCGATGGAGGCCCAGATTCGTCT CCAGTTCAATTTTCCTGTCCAACCAGTCAAGAAAATAACTATTATGCAAAACGTGTCTGAAGTAATTCAT CCGGTTTTATGGGTTGAAATGGGAGTGGTTCTGAACGGATGGTTTCTCCGAATGATTAAAACTTTCTTTT ACTTCCTCACAGCTTTAGAAGTTATGAAATACATCTCGCTGGTCGCAAGTCTCCTTGGAACGGCCTACGG AgGCTACCACTTGTACAAAAACAAGAAACTTTACTCGTTCAAGGAAAATATTGTACTTAGCTCAAAATAA TTTATTTAATTAAATCAAATGATGGC

\section{$\underline{\text { Amino acid sequence }}$}

>MLSYKKITIISACCFVVTIIFGFVAYEPIFEYAIRDQISLHRRNFVRQFYLKYPIPLDFRVNFFNVSNP DEVENGGVPVLSEVGPYCYDLYKERIDVEDNEAEDSLTYTPYDIYLFNQERSGNLSQDDYVTI IHPLVVS LVNYVSVKTPHYLQFLNDALGFLFPEKS I FLTAKVRDI LFDGML INCTSRDFTAMAVCTQIRTKIPGIQF ESKDYLKYALLGQQNGTLPTRITVLRGIKESENLGKLVAVDNVTKSDFWSNEECNEYKGTDGWIFPPFSG RLKTIWMHATTLCQNIHADFVGPATSNGFAVNKYYSDFQNICTNCSLQEPCLPEGLIDVTKCLTAPIYIS LPHFLRSDESLIRGVKGLNPDTESHITRILLEGTLSLPMEAQIRLQFNFPVQPVKKITIMQNVSEVIHPV LWVEMGVVLNGWFLRMIKTFFYFLTALEVMKY ISLVASLLGTAYGGYHLYKNKKLYSEKENIVLSSK* 


\section{Supplemental}

\subsubsection{TcSNMP1d}

\section{cDNA sequence}

>CACTGTGGCCATGCACAAGTCGAAGAAAATACTAGCCGGTTCGGTAGTCGCGACCCTTTTCGTTGTGGT TTTCGGTTTTATTACCTTCTCAGGTTTCGTCAAGTTTGCGGTTCGAGACCAAACTGCGCTTAGGCGGAGA AATATAATGAAGAAGGTGTACTTGAAAATCCCCATGCCGTTGGATTTTCGCGTCTATTTTTTCAACATCA CGAATCCGTCTGAAGTGCAAAACGGCGAACTGCCGGTGGTTAAAGAAGTCGGACCGTACTGTTACGATGC CTTTAAGGAAAAAATCGACGTTTTGGAGAACGAGGGCGAGGACAGTCTCACTTACACCCCCTACGAAACT TATTTTTTCAATCAGGACAAATCCGGGCGCTTGACTGCCGACGATTACGTGACGGTTCTCCATCCCCTGA TTGTGGGTATTGTTAATACCGTTTCAAGAGATTCACCGCCTTTGTTACCCATCGTGGACCGAGCCATTAA ATCAATATTCAAAGACCCGCAAAATATCTACATCACAACGAAAGTTCGGGACTTTTTGTTTGACGGAATG ACGATTAACTGCAAAGTTCAGGACTTTTCGGCAACGGCTGTTTGCACGCAACTTAAAGCCCAGATTCCGG GATTGATTGAAATTGAGAAAAATGTTTACAAGTTTTCGATTCTAGGACCGCGAAATGGCACGTTACCGAA CCGGTATAAAGTCTTCAGAGGGATGAAAAAATGGCACGAATTGGGTCGTTTAGTTGAGGTGAATCACGAA AAGGAGTCAACAGTGTGGTCCACGAAAAAATGTAACAGATTTAGGGGAACTGACGGTTGGATTTTCCCCC CGTTTATCGACAGGGAAGTGGGTTTTTGGACGTATTCTTCCGATTTGTGCCGCAATATGCACCTCGTCTT CGTCGAAGAGACGAGTTTTCACGGAGTTCCCGCTGAGAAATATTACGCCGATTTGGGCGACATGTCTTCA AACCCTGACGAGAAGTGTTACTGTCCGAAGACTTGTCTCCCGAAAGGCATGATGGACTTGACCCGCTGTA TGGGGGTGCCCATCTACGCCACCCTGCCGCACTTCCTCCGAGTGGACAAAGAAGTGAGGAGAACTGTCAG AGGTTTGAAGCCAATCACCGACGAACACATCGTGCGAGTGATAATCCAACCTTTGTTGGGAACTCCACTA GAAGCACAAAAGAGAATGCAATTCAATTTGCCGATCCAACCTGTCAAGAAAATTAGTTTAATGAAAACGT TGCCTCAGGCCCTACATCCCATTTTCTGGATCGAGGAAGCGATCGTTTTGGAAGGACCGCTGTTTAAAAT GATTAAAGTCGTTTTCGTTGCTTTGAAAGTGTTTGATGTTGTTAAGTATTGCTTGCTGGCGGTTTGTCTC GCGTTTGTGGCTTTTGGGAGCTACTTGTGCTACAAGGAGAGGAAGCAGAAGAAACAGGCGGTGACGCCCG TGTCCAAAACTGCCAGTACTGAGCATTTACTTAAGTCTGAACAAAACGCTTTTGAAGAAAATAAAAAAAT TGAAGACTATATTAAAAATGACGAAAAAATTGGAAAATTTTAACGTAATTTCAACACACTTAGTAGAATC ACTGGAACTTCATCAAAGAGCACAAAATTACTCAACAACTGCCTATTATACATAAATAAATTTTTTGGTT ACATCGT

\section{Amino acid sequence}

$>$ MHKSKKILAGSVVATLFVVVFGFITFSGFVKFAVRDQTALRRRNIMKKVYLKIPMPLDFRVYFFNITNP SEVQNGELPVVKEVGPYCYDAFKEKI DVLENEGEDSLTYTPYETYFFNQDKSGRLTADDYVTVLHPLIVG IVNTVSRDSP PLLPIVDRAIKSIFKDPQNIYITTKVRDFLFDGMT INCKVQDFSATAVCTQLKAQI PGLI EIEKNVYKFS ILGPRNGTLPNRYKVFRGMKKWHELGRLVEVNHEKESTVWSTKKCNRFRGTDGWIFPPFI DREVGFWTYSSDLCRNMHLVFVEETSFHGVPAEKYYADLGDMSSNPDEKCYCPKTCLPKGMMDLTRCMGV PIYATLPHFLRVDKEVRRTVRGLKP ITDEHIVRVI I QPLLGTPLEAQKRMQFNLP I QPVKKISLMKTLPQ ALHPIFWIEEAIVLEGPLFKMIKVVFVALKVFDVVKYCLLAVCLAFVAFGSYLCYKERKQKKQAVTPVSK TASTEHLLKSEQNAFEENKKIEDYIKNDEKIGKF* 


\subsubsection{TcSNMP1z}

\section{cDNA sequence}

>ACAAAACCCTACGTTGATTTAACACTAGCACGCAAAATGCGCCTAAGTACGCCCGTAATTGTGGCCATT TCAGGCTTCTGTATCTTACTCTTAGCCCTACTTTGCTCAAGTGTGATTTTTCCTGAACTGGAAAACTACT TAATAAGTAAGGAAACCGCGTTCGTAGAAGGGAGCAAGACATTTGAGACCTGGAAAAAAATACCGTTCCC GTTCAAATTAAAAATTTATTTTCTCAATGTGACCAACGTTGATGATTTCCAAATGGGGGCCAAACCAACT TTTAGGGAAATAGGGCCCTATGTTTACGACGAATTTCGGGAACGCGAAGTTTTAGCAGTTAGTGAAGACA ACGACACTGTTAGGTATAATCAAAAAAAGACCTATTTTTTCAATAGAGAGGACTCAGGGTGTCGGACGGA AGAAGATGTAGTAACGATAATTAACATCGCCGCTGTGGGAATCGCTCATAAAATTTATAAACTCGCACCA GACGCGATGGATATAGTAAACGATGCTTTGCCATTCCTTTACCCTGGCATTAAGAACGTTTTTGTGACTA ACACAGTCCAAAACATTTTATTCGACGGTGTTACAATGTCTTGCGGATCGGACGAAGTGGCAATGATTTG TGATGGGTTAAAGAAACGACGTCCACCCAGCATTCGACCAGCTGACAACAACAAGGACTATTTGGTTGCA ATGTTCCATCATATGAATGGGTCCGTTGACGGTCCTTACGAGATACAACGTGGGCTCAAAGACTCAAGCA AAGGTCAGGTGGTCGGTTTCAAAGACAACAACATGTTGACGCTCTGGACCGGCGACTGCAACACAATTCA AGGAACCGACCTCACCCTCAACCCAAACTTGAACGACTTGCCCCCGAAAATATATTTTTTCGCATCTGAC TTCTGCCGCTCATTTTCCGTAAAATTCGACAAAGAGTTGGTTTATTTGGGGCTTAAAAGTTACAAATTCA AGAACAGTAATTTATTCCACATTGAGAAAAATTGTTTCTGTGACAAGAACCCCGAAAATGAGGTGCCGGG ATGTACACCGGCTGGAACAATGGATGTGAGTCCTTGTACCGGATCCTCGGTTGTACTCTCGCAGCCGCAT TTTTTAAACGCTGAAAAATCGCTACTTGACTATGCCCAAGGCCTCGCACCTAACGAAAATAGGCATGGGA CCTTTATCATAATGGAACCGAAAACCGGTTTGGCACTTGTCGTCAAAACACGGTTTCAAATGAACGTTTA TTTACAGGATTTTGAAGATGTTGACCTACTTGCAAATGTTAGCGCCGGCTTTTTCCCAATGCTTTGGCTC GAGAACGGCGCTGAGGTTCCCCATGATATCGTGGACGAGATTCGCGAAAATTTCAACAAACTGATGGTTT TTGACGCGGTTAAGTACTTCCTGATCGTGCTCGGGGCGATTTTGTTAGCCGTTTCCATAATGCTAGCGAT GAGTCAAGACAAAGTGTGGTGTTTCACCAACAAAAATAACTCAATTGCTAGCGACAACCAAATGGCGAAA ATTATGAATCTGAACGCAAATGCTGCACTTGCCCGGAACTGGGAAAATATCAACAACGCCACTTGGAAAA TCGTGGAATAGAGCACTTACTTTACATAAGGTGTGACCTAGATAGGAAAAAAGTGTAAACACCCGATAAA AATTCCAATTTATTCAAACATTTCGACGAACAACAAAGTTGAGATTAATCTAAATCACATTTAAACTAG AAAAACAATTATTTAGTTAAGTACCTGCGTGTTTACTAACAAATGTTTGAAAGGAACTCCTTCACTTTTT TGTGCACATTTTTTATTAAAAATCACAAATAAATAATTCGGT

\section{$\underline{\text { Amino acid sequence }}$}

>MRLSTPVIVAISGFCILLLALLCSSVIFPELENYLISKETAFVEGSKTFETWKKIPFPFKLKIYFLNVT NVDDFQMGAKPTFREIGPYVYDEFREREVLAVSEDNDTVRYNQKKTYFFNREDSGCRTEEDVVTI INIAA VGIAHKIYKLAPDAMDIVNDALPFLYPGIKNVFVTNTVQNILFDGVTMSCGSDEVAMICDGLKKRRPPS I RPADNNKDYLVAMFHHMNGSVDGPYEIQRGLKDSSKGQVVGFKDNNMLTLWTGDCNTIQGTDLTLNPNLN DLPPKIYFFASDFCRSFSVKFDKELVYLGLKSYKFKNSNLFHIEKNCFCDKNPENEVPGCTPAGTMDVSP CTGSSVVLSQPHFLNAEKSLLDYAQGLAPNENRHGTFI IMEPKTGLALVVKTRFQMNVYLQDFEDVDLLA NVSAGFF PMLWLENGAEVPHDIVDEIRENFNKLMVFDAVKYFLIVLGA ILLAVS IMLAMSQDKVWCFTNK NNS IAS DNQMAK IMNLNANAALARNWENINNATWKIVE* 


\section{Supplemental}

\subsubsection{TcSNMP2}

\section{cDNA sequence}

>AAATGGGGAGGCGCAAAATGGGGTGTTCATGTTGTACCATCAAAGTATTACTAGTTTGTGTAGTAATCA GTGTAGCCCTTTTGATAGTGTCCCTAGCGCTAGCTTTTAAGGTATTTCCCGACCTCCTGGAATCTGAAGT AAATAAGGCTGTTAGGCTCGAGGATGGCACTAAACAGTATGACAGATTTGTTGAACTACCGTTTCCAGTT GACTTCAAAGTGTACCTCTTTAACGTCTCAAACCCCCAACAAGTTCTAGATGGAACTGAAAAACCTAAAC TCGAGGAAATTGGACCCTTTGTGTACAAGCAATACAGGAAAAAGACAATACTGGGCAAAAATGAGGAAGA AGACACAATTTCATACACACAAAAGGAAACTTTCGAATTTGACGCCGAGGCATCTAAACCGCTAACAGAA GACTCAGTTGTAACGGTTTTGAATCCCGCATTGATGTCAATTTTTCAATTGGCGGAGGGTCTTCACTTGG CCGGGGCCGCAGACACTTGCACCAAGCAAACATTTGAGGCCAACCAAGGCAAAGTTTTTATAGAAGCAAA CGTGCGAAAACTGCTGTTTGACGGCTTTAGTTTCTGCAAGAACACTTCGCCTGGAATATGTGGCCTTGTA AACGACCTAATTTGTGCGATTGCAGCGACGAAAAGAAATTCGGACCTTGTCTTGCCCGACTGTTCTTTGA TATTTTCCTATTTGAACTACAAACGAAAGCCGGATGATGGCAAATATACCGTGAAGAGAGGTCTCACCAA CATTGAAAAACTGGGACATATTGTGGCATGGAATGACAGTTTGTATACGAAATTTTGGGGGGAAGGCACC ACCTGCAGTGAAGTTAAAGGGACCGATTCGACCCTTTATCCCCCTAGAGTTACAACAGATAGTGCATTTT ACATCTATTCGACGGACATCTGCAGGTTTGTGGAAATCAATTACAAAGGAGAAGAGAGTTACAAAGGCAT CGATGGCTATTTATTTGAAACCAGTGAGGACACTTTGCGAAGCTCCGCCCCTGAAGAAGACTGCTATTGC AGTAAATTGAGCAGAGACATGGAAGGCAAAAAGAGTTGTTTCTTGGATGGAGTGATAGACATGCAAACTT GTTTCGGAGTGCCAGTTCTTTTCTCATTCCCACACTTCTTGTGGGCCGATAACAAGTACTTAAGTGCAGT GGAAGGACTAAACCCTGTCGAGGAAAAACACAAAACGTATCTCGTTGTCGAGCCAAATACTGGAACTCCT CTGAAAGGAATGAAAAGGATTCAACTGAATGGAGTCATTAGGCCGATTGTTGGAATAAAATCAATGTTGC AAACCAAGCGAGCTTTATTGCCGCTTTTGTGGATAGAAGAAGGAGTGTCTCTGCCCCAAAAATACGTCGA TGAACTAAAATCGACCTACTTTGACAAGGTTCAGATAGTCGATGGTGTTAGATACGCTCTTATAGTAATA TCAGCCATATTGGTTGGTGCTTTTGGAATTATTATTTTACGAAAACGTTCACACGCTAAACACCATGTAT AATAATAGGTGCGCACAAATTAAATCATATATTTGTGTTGAAATACAATCTTACTCAGT

\section{$\underline{\text { Amino acid sequence }}$}

>MGRRKMGCSCCTIKVLLVCVVISVALLIVSLALAFKVFPDLLESEVNKAVRLEDGTKQYDRFVELPFPV DFKVYLFNVSNPQQVLDGTEKPKLEEIGPFVYKQYRKKTILGKNEEEDTISYTQKETFEFDAEASKPLTE DSVVTVLNPALMS I FQLAEGLHLAGAADTCTKQTFEANQGKVFIEANVRKLLFDGFSFCKNTSPGICGLV NDLICAIAATKRNSDLVLPDCSLIFSYLNYKRKPDDGKYTVKRGLTNIEKLGH IVAWNDSLYTKFWGEGT TCSEVKGTDSTLYP PRVTTDSAFYIYSTDICRFVEINYKGEESYKGIDGYLFETSEDTLRSSAPEEDCYC SKLSRDMEGKKSCFLDGVI DMQTCFGVPVLFSFPHFLWADNKYLSAVEGLNPVEEKHKTYLVVEPNTGTP LKGMKRIQLNGVIRPIVGIKSMLQTKRALLPLLWIEEGVSLPQKYVDELKSTYFDKVQIVDGVRYALIVI SAILVGAFGI I ILRKRSHAKHHV* 


\subsection{Comparison of the full-length TcSNMP cDNA sequences with the}

\section{respective in silico annotation}

TCSNMP1a_cDNA TCSNMP1a_calc

TcSNMP1a_cDNA TCSNMP1a_calc

TCSNMP1a CDNA TCSNMP1a_calc

TCSNMP1a_cDNA TCSNMP1a_calc

TcSNMP1a_cDNA TCSNMP1a_calc

TCSNMP1a_CDNA TCSNMP1a_calc

TCSNMP1a_cDNA TCSNMP1a_calc

TCSNMP1a_cDNA TcSNMP1a_calc

TCSNMP1a_cDNA TCSNMP1a_calc

TCSNMP1a_cDNA TCSNMP1a_calc

TCSNMP1a_cDNA TcSNMP1a_calc

TCSNMP1a_cDNA TCSNMP1a_calc

TcSNMP1a_cDNA TCSNMP1a_calc

TCSNMP1a_cDNA TCSNMP1a_calc

TCSNMP1a_cDNA TcSNMP1a_calc

TCSNMP1a_cDNA TCSNMP1a_calc

TCSNMP1a_cDNA TcSNMP1a_calc

TCSNMP1a_cDNA TCSNMP1a_calc

TcSNMP1a_cDNA TCSNMP1a_calc

TcSNMP1a_cDNA TCSNMP1a_calc

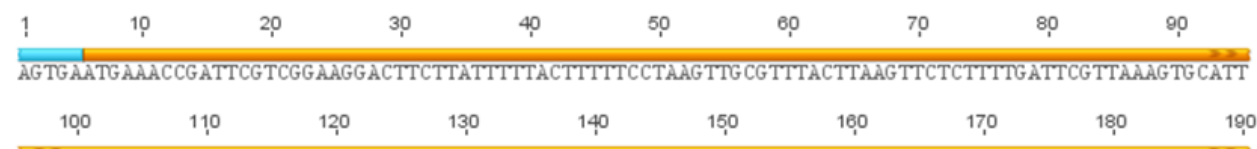

TATCATTGTTACACGAAGAAGAAAACTAAAAAGGAAATGCEGCIGCCGGTCAAAATCGCGATCEGATGCACGATTGGGCTCETTGTATCATTGT

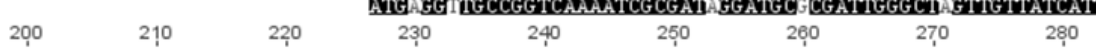

IITCGG ATTTATTGCTITCCTAAAATGATCAAAGGCAAAGTGAAGAGTATGATAAATTTGAACAAGGG GAGCGAAATCCGCCAAATGTTCGTCA ITTCGGATTTATTGCTTTTCCTAAAATG ATCAAAGGCAAAGTGAAGAGTATG ATAAATTTGAACAAGGG AGCG AAATCCGCCAAATGTTCGTCA $\begin{array}{llllllllll}290 & 300 & 310 & 320 & 330 & 340 & 350 & 360 & 370 & 380\end{array}$ AAGTCCCGTTCGCTCTAGACTITAAAATTTATATGTTTAACGTAGCAAATCCGATGGATGTCCAGAAGGGTGCTITGCCTGTCCTAAAAGAAGTG AAGTCCCGTCGCTCTAGACTTTAAAATTTATATGTTTAACGTA CAAATCCGATGGATGTCCAGAAGGG TCTTTGCCTGTCCTAAAG EAAGTG

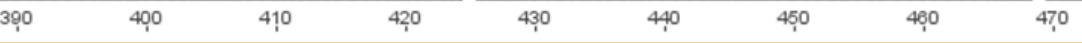

GGACCGTTCTG TTCGA GAATGGAAGGAGAAGGTTGACCTTGACGATAACGACGACGAGGATGTCATGTTTTACAATCCCAAGGACACGTTTTA

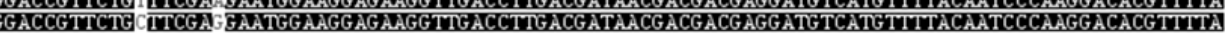

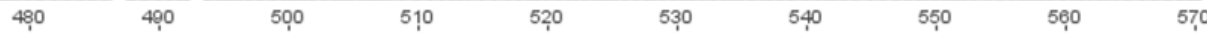

CAAAACTAACGGCCTGGATGCCTCGACGGAAGCCAGATGATTACAATGGCACATCCTTTAATACTCGGGATGGTGAACACAGTGGTGCGGACTA CAAAGC AACGGGCCTGGATGCCTCGACGGAAGCCAGATGATTACAATGGCACATCCTTTAATACTCGGGATGGTGACACAGTGGTGCGGACTA $\begin{array}{ccccccccc}580 & 590 & 6010 & 610 & 620 & 630 & 640 & 650 & 6100\end{array}$ AACCAGGCGCCATTTCCTTGATTAGCAAAGCCATTAATTCCATTTACGGCAATCCTGATTCTATTTTTATG AC I GCTTCGGCIATGGACATCCTG
AACCAGGCGCCATTTCCTTG ATTAGCAAAGCCATTAATTCCATTTACGGCAATCCTG ATTCTATTTTTATG AC GCTTCGGCA ATGGACATCCTG

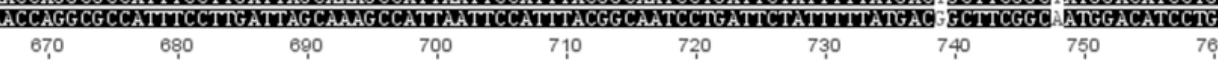
$700^{0}$
$710^{\circ}$
720

TTTGATGGTGTTETAATTAAATGCGGGGTCAAAGATTTCGCCGGAAAGGCCGTTTGTTCGCAGCTGAAAGAAGCACCAGACTTGAGGCATGTCGA ITTGATGGTGTLTAATTAAATGCGGGGTCAAAGATTCGCCGGAAAGGCCGTIGTCGCAGCTGAAGAAGCACCAGACTTGAGGCATGTCGA $\begin{array}{lllllllll}7770 & 78,0 & 7910 & 8090 & 8190 & 820 & 830 & 840 & 850\end{array}$

CGAAAATATTTGGCGTTTTCATTTATAGGGCCTAAAAATCAACTCCCGGGAAGAGATTTAAAGTTCTGAGGGGCGTCAAGAATCGCACGATG

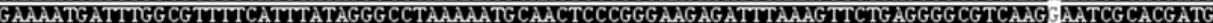
860
890
900
$910 \quad 920$
$930 \quad 940$

DCCG

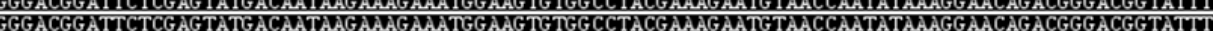

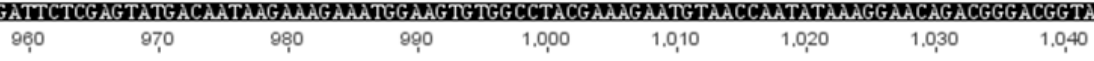
CCCCCTTATCTCACAAAGG AGGAAGGTCTTGCCTCCTATGCGCCCGACTTGTGCCGTTCGTTAGTTGCCGTCTACAGTGGA GACACAAAATACGA
CCCCCTTATCTCACAAAGGAGGAGGTCTTGCCTCCTATGCGCCCGACTTGTGCCGTTCGTTAGTTGCCGTCTACAGTGG EACACAAAATACGA

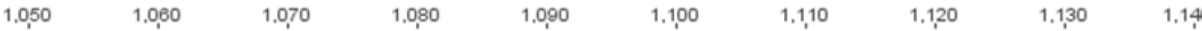

CGGTATTCCTGT ICGAATATACACGGCGACTTTAGGCGACATGTCCAAAAATGCAGACGAGAAGTGTTACTGCCCCACACCTGACACTTGCCTGA CGGTATTCCTG 1 CGAATATACACGGCGACTTTAGGCGACATGTCCAAAAATGCAGACG AGAAGTGTTACTGCCCCACACCTG ACACTTE ICTG

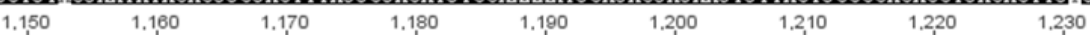

AGAAGGGCATGATGGACCTGTTCAAGTGTGCAGGAGTTCCTGTCTATGTCTCACTACCACATTTCTACGAATCGGACGAAAGCTACGTCAAAGGA W
1,240 1,250
$\begin{array}{ll}1,260 & 1,270\end{array}$
1,290
1,300
1,320
1,330

GTCGTCGGCCTTAACCCTAACAAGAAAGACCACGGAATTCAGATTTTGTTCGAATCGACAACTGG GGTCCTGTCAAAGCGCGAAACGCCTCCA HTCGTCGGCCTTAACCCTAACAAGAAAGACCACGGAATTCAGATTTTGTTCGAATCGACAACTGG GGTCCTGTCAA GCCGCGAAACGCCTCCA $\begin{array}{lllllllll}1,3,40 & 1,350 & 1,360 & 1,370 & 1,380 & 1,3,90 & 1,400 & 1,4,10 & 1,4,20\end{array}$

GTTCAACATGCCCCTGGAGCCGAATCCAAAATTGCCAATTTTTGCAAACTTGCCCAACACAGTTTTGCCACTGTTCTGGGTGGAGGAAGGCGTGG TTCAACATGCCCCTGG AGCCG AATCCAAAATTGCCAATTTTTECAAACTTGCCCAACACAGTTTTECCACTGTTCTGGGTGG AGG AAGGCGTGG $\begin{array}{llllllllll}1.430 & 1.440 & 1.450 & 1.460 & 1.470 & 1.480 & 1.490 & 1,500 & 1,5,10 & 1,520\end{array}$

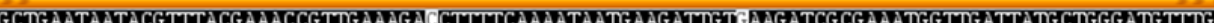

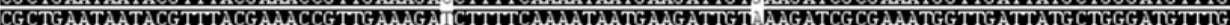
$\begin{array}{lllllllll}1.530 & 1,5,40 & 1,550 & 1,560 & 1,570 & 1,580 & 1,590 & 1,600 & 1,610\end{array}$ BGGGGTTTGGGAGCCGCCGGTTATCTCTACTTTTCCAAAAAAGGGGAAGCGAATATTACGCCGGTACATAAGGTTAAACCGGCCGAAAATGGGGT
GGGGGTTFGG AGCCGCCGGTTATCTCTACTTTCCAAAAAGGGGAAGCGAATATTACGCCGGTACATAAGGTTAACCGGCCGAAAATGGGGT

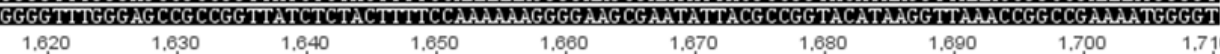
AGCACTCTCGGGGT AAGTCAACCATGCCATGTCTGATAACGAGATAGAGAAATACTG ATG ATTTGTTTAGGCAGGTTCTGTCTAGTCTTGT

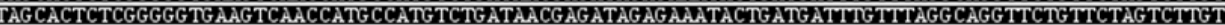
$\begin{array}{lllllllll}1.720 & 1,730 & 1,7,40 & 1,750 & 1,760 & 1,770 & 1,780 & 1,7,90 & 1,800\end{array}$

IGGAAGATTGCTAGTTTATTITATATATTTTTGTATT AAAGCGGTGTGTTAGTTAACGATTAGTTAATTATTTGTGCACGATTTTTAACTGT

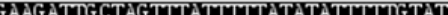

$\begin{array}{llllllllll}1.810 & 1,820 & 1,830 & 1,840 & 1,850 & 1,860 & 1,870 & 1,880 & 1,890 & 1,900\end{array}$ TTTAGTCTGTGATTAGTGATATGTATGAGATATGGTTAGTTTATTAACTTTAGAAAAATGTTATATTGACAGGGTATGCTAGTCAGAAAAAT $\begin{array}{llllllll}1,9,10 & 1,920 & 1,930 & 1,940 & 1,950 & 1,960 & 1,970 & 1,980\end{array}$ AAATTATATTATTTGTAATTTTGCTTTTATTTCAAATACACTTTATCTTGAAAAAAAAAAAAAAAAAAAAAAAAAAAAGT

Figure S1 Comparison of the obtained TcSNMP1a cDNA sequence with the in silico annotation 
Shown is the cDNA sequence received from RACE-PCR (TcSNMP1a_cDNA) compared with the computer calculated annotation (TcSNMP1a_calc). Identical nucleotides are depicted with a black background. Numbers show the nucleotide position of the obtained cDNA sequence. The ORF (open reading frame) of the obtained cDNA sequence is labelled by a yellow line above the sequence and the $5^{\prime}$ and $3^{\prime}$ UTR (untranslated region) with a blue line.

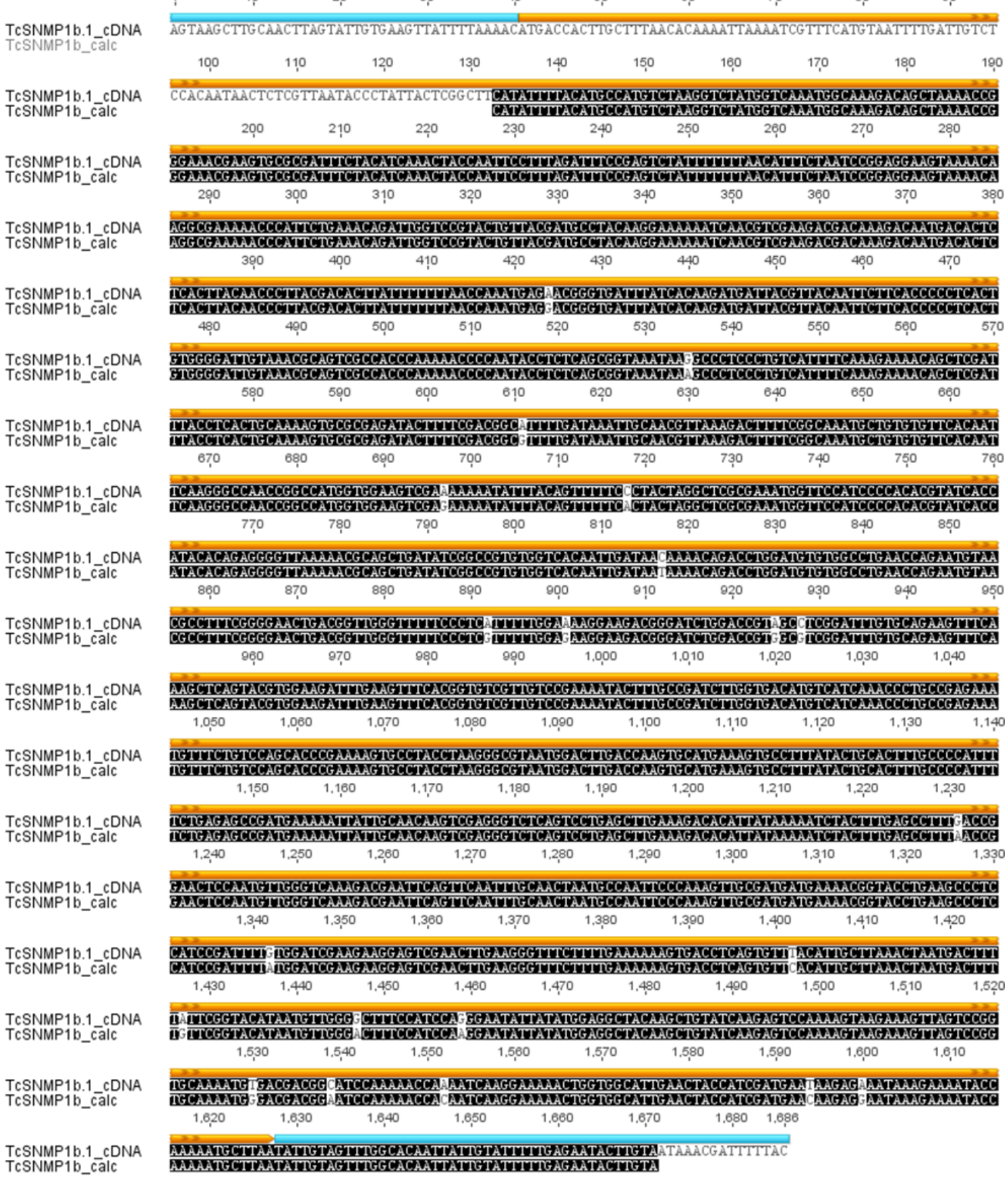

Figure S2 Comparison of the obtained TcSNMP1b.1 cDNA sequence with the in silico annotation

Shown is the cDNA sequence received from RACE-PCR (TcSNMP1b.1_cDNA) compared with the computer calculated annotation (TcSNMP1b_calc). Identical nucleotides are depicted with a black background. Numbers show the nucleotide position of the obtained cDNA sequence. The ORF (open 
reading frame) of the obtained cDNA sequence is labelled by a yellow line above the sequence and the $5^{\prime}$ and $3^{\prime}$ UTR (untranslated region) with a blue line.

TcSNMP1c_cDNA TCSNMP1c_calc

TcSNMP1c cDNA TcSNMP1c_calc

TCSNMP1C_CDNA TCSNMP1c_calc

TcSNMP1c_cDNA TCSNMP1c_calc

TCSNMP1C_CDNA TCSNMP1c calc

TCSNMP1c_cDNA TCSNMP1c_calc

TCSNMP1C_cDNA TCSNMP1c_calc

TCSNMP1C CDNA TCSNMP1c_calc

TCSNMP1c_cDNA TCSNMP1c_calc

TCSNMP1C CDNA TCSNMP1c_calc

TcSNMP1c_cDNA TCSNMP1c_calc

TCSNMP1c_cDNA TcSNMP1c_calc

TCSNMP1c_cDNA TCSNMP1c_calc

TCSNMP1c_cDNA TCSNMP1c_calc

TcSNMP1c_cDNA TCSNMP1c_calc

TCSNMP1c_cDNA TCSNMP1c_calc

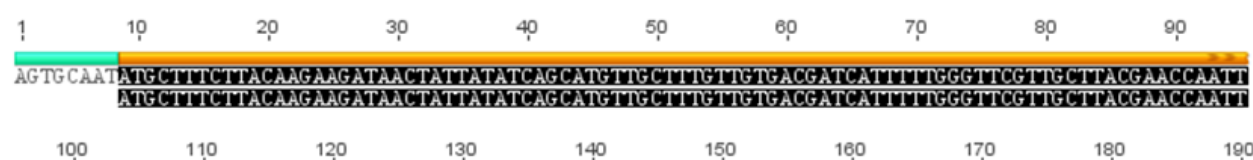

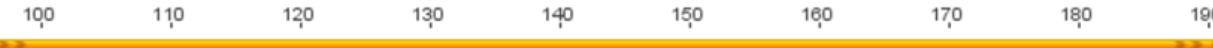

II GAATATGCAATTAGAGACCAAATTTCTCTGCATCGGAGGAACTTTGTGAGACAGTTITACCTAAAG TACCCAATACC ITGG AITTCCGTGT

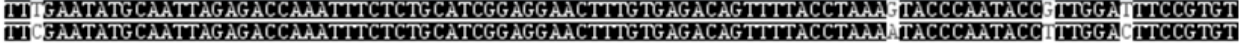

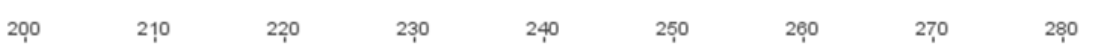

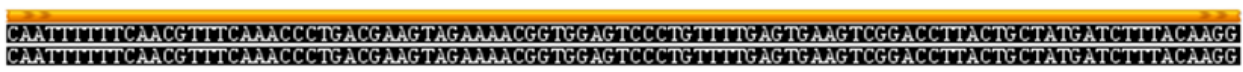
$2900 \quad 3000 \quad 310 \quad 320 \quad 330 \quad 340 \quad 350 \quad 36,0 \quad 370 \quad 380$ AGAGAATAGACGTGGAGGACAACGAGGCCGAGGACAGCCTCACCTACACCCCTTACGACATTTACTTATITAATCAGGAGAGATCGGGAAACTTA AGAGAATAG ACG TGGAGGACAACG AGGCCGAGG ACAGCCTCACCTACACCCCTTACG ACATTTACTTATTTAATCAGG AGAG ATCGGG AAACTT

$\begin{array}{llllllll}3900 & 4090 & 410 & 420 & 430 & 440 & 450 & 460\end{array}$

AGTCAGG A I GATTACGTTACAATTATTCACCCCTTGTCGTGAGCCTTGTCAATTACGT GCAGTGAAGACGCCTCACTATTTACAGTTCTAAA

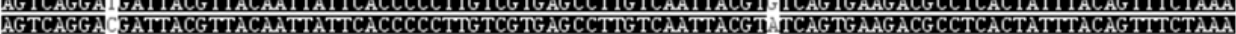

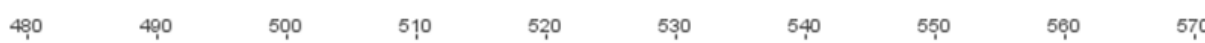
CGACGCACTTGGATTTCTGTTCCCTGAAAAATCCATCTTTCTTACCGCCAAAGTCAGAGATATTCTATTTGACGGAATGTTAATAAACTGCACGT
CGACGCACTTGG ATTTCTETTCCCTGAAAATCCATCTTTCTTACCGCCAAGGCAGAGATATTCTATTTG ACGGAATGTTATAAACTGCACGT

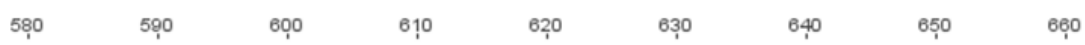

CTCGGGATTTCACAGCGATGGCAGTTIGTACACAAATACGAACAAAAATTCCCGGAATACAGTTTGAAAGTAAAGATTATTTGAAATATGCGCTC

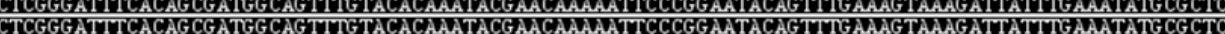

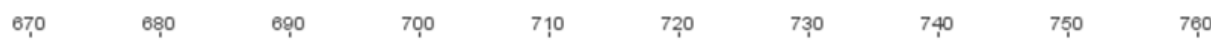

CTTGGACAACAAAATGGACTITACCTACTA BATAACGGTTTTGAGGGCATAAAAGAGTCAGAGAATTTGGGCAAATTGGTGGCTGTTGATAA

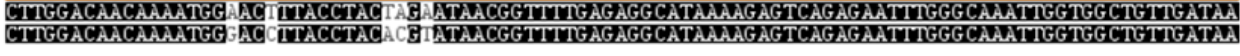
77,0
$780 \quad 790$
8010
810
$820 \quad 830$
840

850

CGTGACAAAGAGTGATTTCTGGTCAAATGAABAATGTAATGAGTATAAGGGCACCGATGGTTGGATTTTCCCCCGTTTTCTGGGAGGTTGAAGA

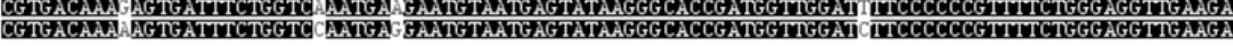

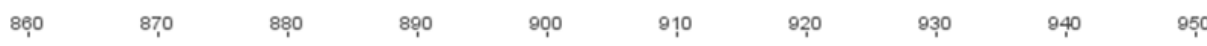

CGATCTGGATGCACGCAACAACACTGTCCAAAATATCCATGCCGATTTCGTGGTCCTGCTACAAGTAACGGTTTTGCAGTAAATAAATACTAC
GATCTG

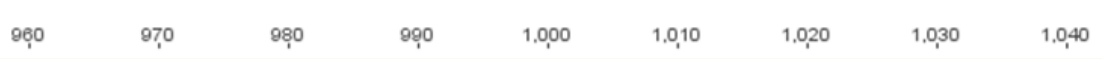

TCTGACTTTCAAAATATTTGTACGAATTGCTCGCTTCAAGAGCCTTGCCTTCCGGAAGGATTAATCGACGTCACGAAATGTCTAACAGCTCCTAT \begin{tabular}{l} 
ICTGACTTTCAAAATATTIGTACGAATTGCTCGCTTCAAGAGCCTTGCCTTCCGGAAGGATTAATCGACGTCACGAAATGTCTAACAGCTCCTAT \\
\hline ICTGACTTTCAAAATATTTETACGAATTGCTCGCTTCAAGAGCCTTGCCTTCCGGAAGGATTAATCGACGTCACG AAATETCTAACAGCTCCTAT
\end{tabular}

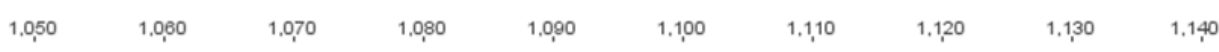

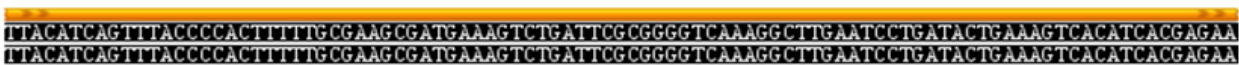

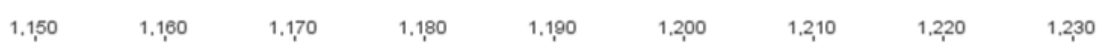

\section{ITCTCCTCGAGGGGACACTGTCGCTTCCGATGGAGGCCAGATTCGTCTCCAGTTCAATTTTCCTGTCCAACCAGTCAAGAAAATAACTATTATG} ITCTCCTCGAGGG ACACTGTCGCTTCCGATGGAGGCCCAGATTCGTCTCCAGTTCAATTTTCCCTGTCCAACCAGTCAAGAAAATAAACTATTATTG

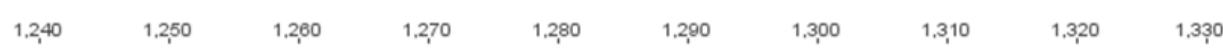
CAAAACGTGTCTGAAGTAATTCATCCGGTTTTATGGGTTGAAATGGGAGTGGTTCTGAACGGATGGTTTCTCCGAATGATTAAAACTTTCTTTTA

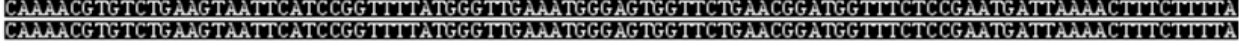

$$
\begin{array}{lllllllll}
1,3,40 & 1,350 & 1,3,30 & 1,370 & 1,3,80 & 1,390 & 1,400 & 1,4,10 & 1,420
\end{array}
$$

CTTCCTCACAGCTTTAGAAGTTATGAAATACATCTCGCTGGTCGCAAGTCTCCTTGGAACGGCCTACGGAGGCTACCACTTGTACAAAAACAAGA
$\frac{\text { CTTCCTCACAGCTTTAGAAGTTATGAAATACATCTCGCTGGTCGCAAGTCTCCTTGGAACGGCC TACGGAGGCTACCACTTGTACAAAAACAAGA }}{\text { CTTCCTCACAGCTTTAGAAGTTATGAAATACATCTCGCTGGTCGCAAGTCTCCTTGGAACGGC IACGGAGGCTACCACTTGTACAAAAACAAGA }}$

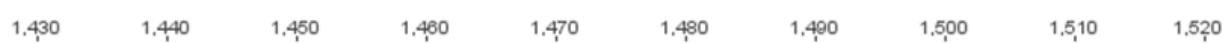

AACTTTACTCGTTCAAGGAAAATATTGTACTTAGCICAAARTAITTITTAATTAAICAAMTGATG

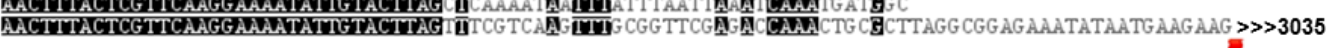

Figure S3 Comparison of the obtained TcSNMP1c cDNA sequence with the in silico annotation

Shown is the cDNA sequence received from RACE-PCR (TcSNMP1c_cDNA) compared with the computer calculated annotation (TcSNMP1c_calc). Identical nucleotides are depicted with a black background. Numbers show the nucleotide position of the obtained cDNA sequence. The ORF (open reading frame) of the obtained cDNA sequence is labelled by a yellow line above the sequence and 
the $5^{\prime}$ and 3' UTR (untranslated region) with a blue line. The red square indicates that the sequence of TcSNMP1c_calc is continued to alignment position 3335 in figure S4.

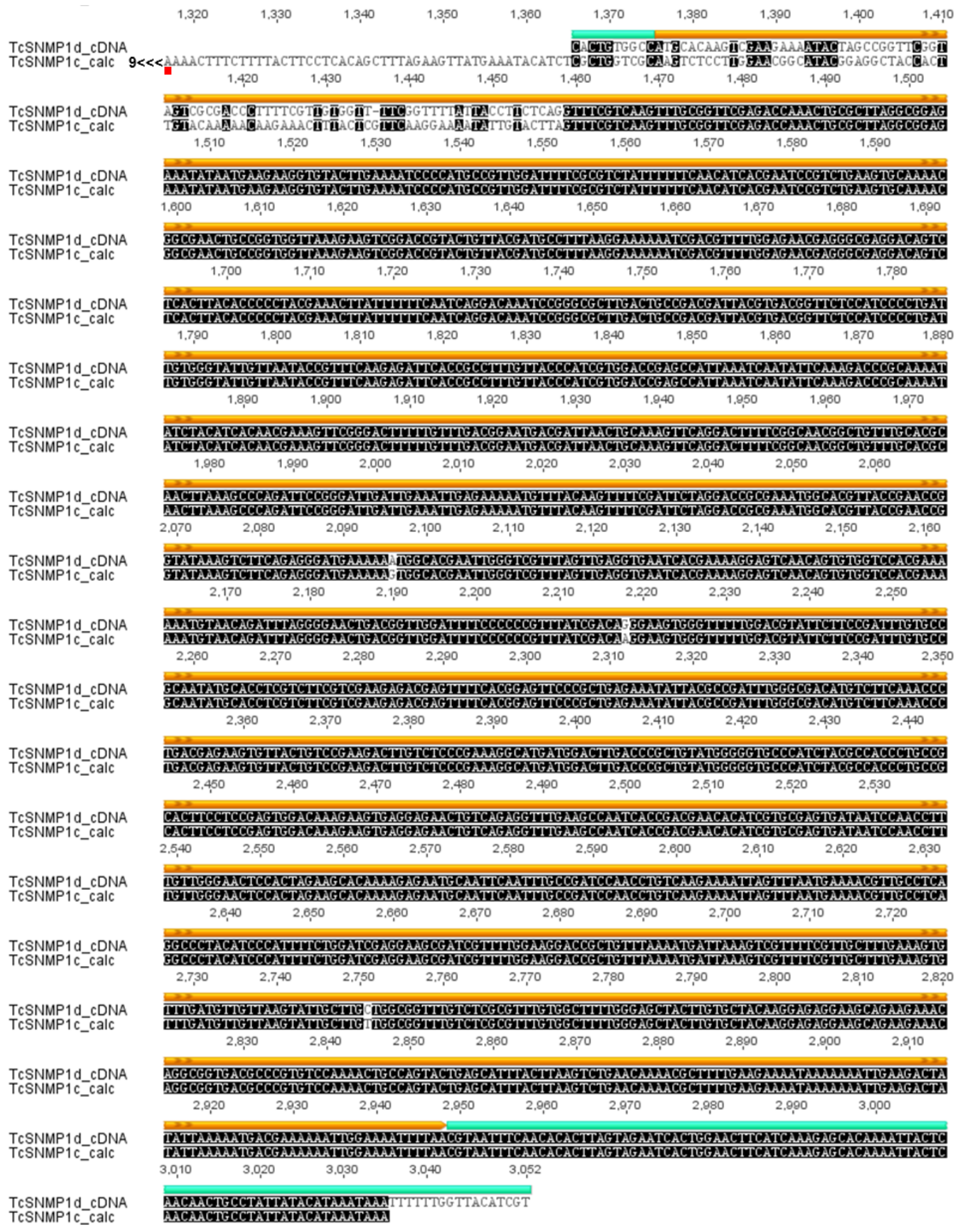

Figure S4 Comparison of the obtained TcSNMP1d cDNA sequence with the in silico annotation

Shown is the cDNA sequence received from RACE-PCR (TcSNMP1d_cDNA) compared with the computer calculated annotation (TcSNMP1c_calc). Identical nucleotides are depicted with a black background. Numbers show the nucleotide position of the calculated cDNA sequence. The ORF (open reading frame) of the obtained cDNA sequence is labelled by a yellow line above the sequence and the $5^{\prime}$ and $3^{\prime}$ UTR (untranslated region) with a blue line. The red square indicates 
that the shown sequence of TcSNMP1c_calc is the continuation of this sequence shown in figure S3, which starts at alignment position 9.

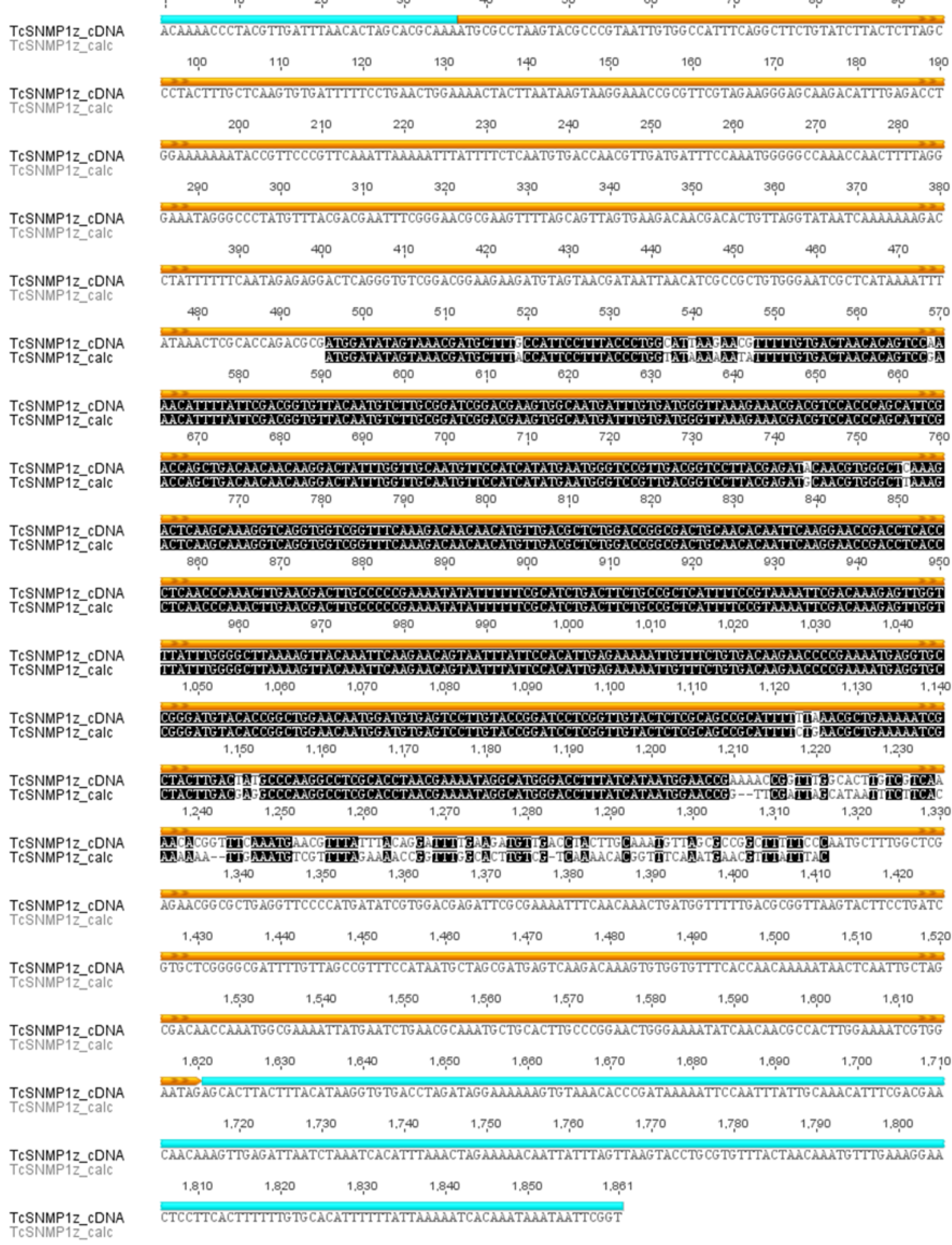

Figure S5 Comparison of the obtained TcSNMP1z cDNA sequence with the in silico annotation

Shown is the cDNA sequence received from RACE-PCR (TcSNMP1z_cDNA) compared with the computer calculated annotation (TcSNMP1z_calc). Identical nucleotides are depicted with a black background. Numbers show the nucleotide position of the obtained cDNA sequence. The ORF (open 
reading frame) of the obtained cDNA sequence is labelled by a yellow line above the sequence and the $5^{\prime}$ and $3^{\prime}$ UTR (untranslated region) with a blue line.

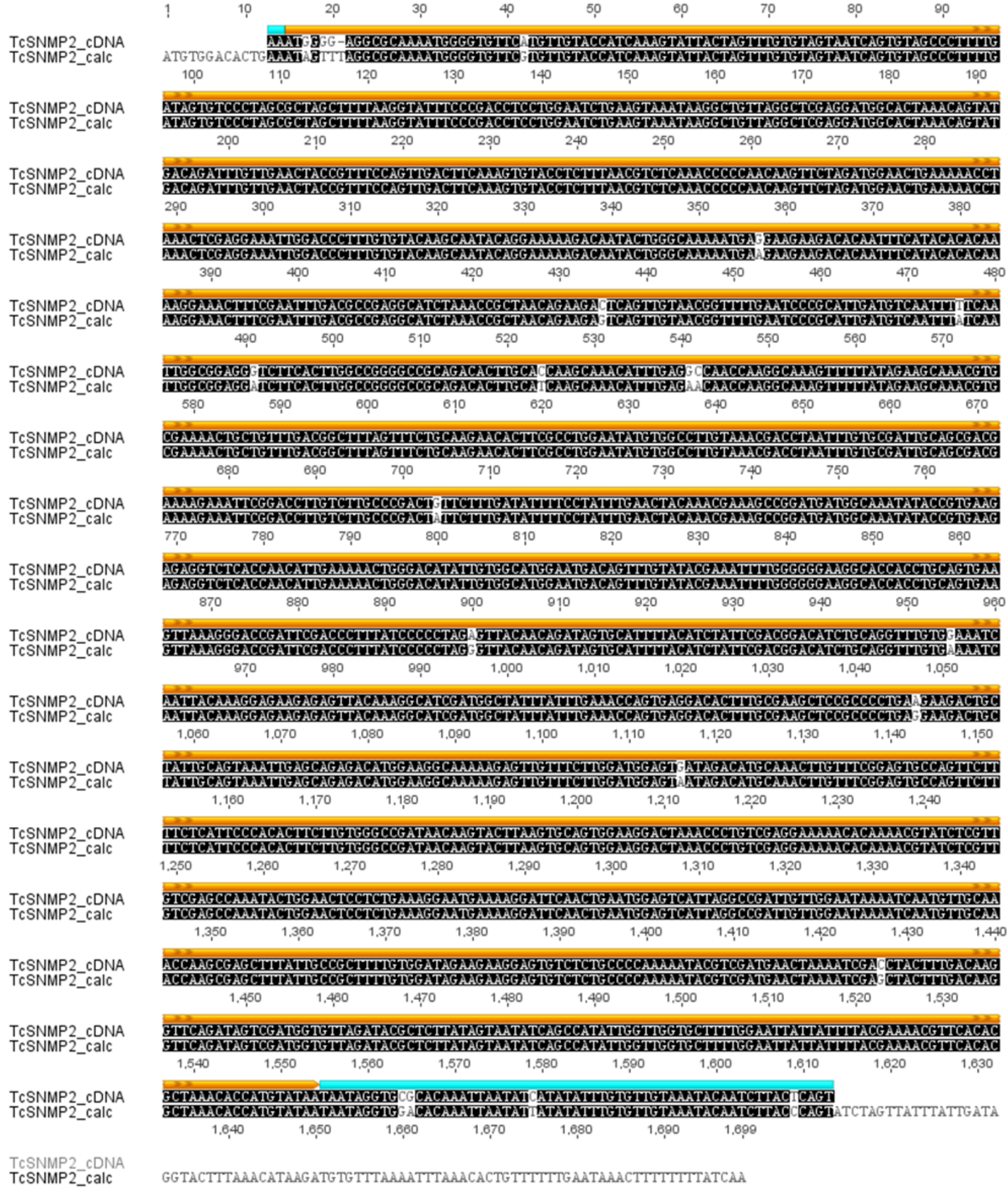

Figure S6 Comparison of the obtained TcSNMP2 cDNA sequence with the in silico annotation Shown is the cDNA sequence received from RACE-PCR (TcSNMP2_cDNA) compared with the computer calculated annotation (TcSNMP2_calc). Identical nucleotides are depicted with a black background. Numbers show the nucleotide position of the calculated cDNA sequence. The ORF (open reading frame) of the obtained cDNA sequence is labelled by a yellow line above the sequence and the $5^{\prime}$ and $3^{\prime}$ UTR (untranslated region) with a blue line. 


\section{Bibliography}

Abdullah, Z.S., and Butt, T.M. (2015). Preferences of the peripheral olfactory system of Western Flower Thrips, Frankliniella occidentalis towards stereoisomers of common plant volatiles. Chemoecology 25, 47-51.

Agarwal, G., and Isacoff, E. (2011). Specializations of a pheromonal glomerulus in the Drosophila olfactory system. J. Neurophysiol. 105, 1711-1721.

Akai, H., and King, R.C. (2012). Insect Ultrastructure (Springer Science \& Business Media).

Akerboom, J., Chen, T.-W., Wardill, T.J., Tian, L., Marvin, J.S., Mutlu, S., Calderón, N.C., Esposti, F., Borghuis, B.G., Sun, X.R., et al. (2012). Optimization of a GCaMP calcium indicator for neural activity imaging. J. Neurosci. Off. J. Soc. Neurosci. 32, 13819-13840.

Alcorta, E. (1991). Characterization of the electroantennogram in Drosophila melanogaster and its use for identifying olfactory capture and transduction mutants. J. Neurophysiol. 65, 702-714.

Andersson, M.N., Larsson, M.C., and Schlyter, F. (2009). Specificity and redundancy in the olfactory system of the bark beetle Ips typographus: single-cell responses to ecologically relevant odors. J. Insect Physiol. 55, 556-567.

Andersson, M.N., Grosse-Wilde, E., Keeling, C.I., Bengtsson, J.M., Yuen, M.M., Li, M., Hillbur, Y., Bohlmann, J., Hansson, B.S., and Schlyter, F. (2013). Antennal transcriptome analysis of the chemosensory gene families in the tree killing bark beetles, Ips typographus and Dendroctonus ponderosae (Coleoptera: Curculionidae: Scolytinae). BMC Genomics 14, 198.

Andersson, M.N., Videvall, E., Walden, K.K., Harris, M.O., Robertson, H.M., and Löfstedt, C. (2014). Sex- and tissue-specific profiles of chemosensory gene expression in a herbivorous gall-inducing fly (Diptera: Cecidomyiidae). BMC Genomics 15, 501.

Angelini, D.R., Kikuchi, M., and Jockusch, E.L. (2009). Genetic patterning in the adult capitate antenna of the beetle Tribolium castaneum. Dev. Biol. 327, 240-251.

Angerer LM, Angerer RC. (1992). In situ hybridization to cellular RNAm with radiolabeled RNA probes. In: Wilkinson DG, editor. In situ hybridization: a practical approach, the practical approach series. Oxford, UK: Oxford University Press; 1992. p. 15-32.

Anton, S., and Homberg, U. (1999). Antennal Lobe Structure. In Insect Olfaction, P.D.B.S. Hansson, ed. (Springer Berlin Heidelberg), pp. 97-124.

Atwal, A.S. (1976). Agricultural pests of India and South-East Asia. $\mathrm{x}+502$ pp. 
Aungst, J., and Spehr, M. (2005). The Tuning Properties of Antennal Lobe Projection Neurons. J. Neurosci. 25, 10339-10340.

Bargmann, C.I. (2006). Comparative chemosensation from receptors to ecology. Nature 444, 295-301.

Barth, J., Dipt, S., Pech, U., Hermann, M., Riemensperger, T., and Fiala, A. (2014). Differential Associative Training Enhances Olfactory Acuity in Drosophila melanogaster. J. Neurosci. 34, 1819-1837.

Benton, R. (2006). On the ORigin of smell: odorant receptors in insects. Cell. Mol. Life Sci. CMLS 63, 1579-1585.

Benton, R., Sachse, S., Michnick, S.W., and Vosshall, L.B. (2006). Atypical membrane topology and heteromeric function of Drosophila odorant receptors in vivo. PLoS Biol. 4, e20.

Benton, R., Vannice, K.S., and Vosshall, L.B. (2007). An essential role for a CD36-related receptor in pheromone detection in Drosophila. Nature 450, 289-293.

Berg, B.G., Galizia, C.G., Brandt, R., and Mustaparta, H. (2002). Digital atlases of the antennal lobe in two species of tobacco budworm moths, the Oriental Helicoverpa assulta (male) and the American Heliothis virescens (male and female). J. Comp. Neurol. $446,123-134$.

Binzer, M., Heuer, C.M., Kollmann, M., Kahnt, J., Hauser, F., Grimmelikhuijzen, C.J.P., and Schachtner, J. (2014). Neuropeptidome of Tribolium castaneum antennal lobes and mushroom bodies. J. Comp. Neurol. 522, 337-357.

Bischof, J., Maeda, R.K., Hediger, M., Karch, F., and Basler, K. (2007). An optimized transgenesis system for Drosophila using germ-line-specific $\varphi \mathrm{C} 31$ integrases. Proc. Natl. Acad. Sci. U. S. A. 104, 3312-3317.

Blomquist, G.J., Vogt, R., and Brody (2003). Insect Pheromone Biochemistry and Molecular Biology: The Biosynthesis and Detection of Pheromones and Plant Volatiles (Amsterdam: Academic Pr Inc).

Bucher, G., Scholten, J., and Klingler, M. (2002). Parental RNAi in Tribolium (Coleoptera). Curr. Biol. CB 12, R85-R86.

Carey, A.F., and Carlson, J.R. (2011). Insect olfaction from model systems to disease control. Proc. Natl. Acad. Sci. U. S. A. 108, 12987-12995.

Carey, A.F., Wang, G., Su, C.-Y., Zwiebel, L.J., and Carlson, J.R. (2010). Odorant reception in the malaria mosquito Anopheles gambiae. Nature 464, 66-71.

Cavener, D.R. (1987). Comparison of the consensus sequence flanking translational start sites in Drosophila and vertebrates. Nucleic Acids Res. 15, 1353-1361. 
Chapman, R.F., Simpson, S.J., and Douglas, A.E. (2013). The Insects: Structure and Function (Cambridge University Press).

Chen, M., Yang, Y., Braunstein, E., Georgeson, K.E., and Harmon, C.M. (2001). Gut expression and regulation of FAT/CD36: possible role in fatty acid transport in rat enterocytes. Am. J. Physiol. Endocrinol. Metab. 281, E916-E923.

Chintapalli, V.R., Wang, J., and Dow, J.A.T. (2007). Using FlyAtlas to identify better Drosophila melanogaster models of human disease. Nat. Genet. 39, 715-720.

Chou, Y.-H., Spletter, M.L., Yaksi, E., Leong, J.C.S., Wilson, R.I., and Luo, L. (2010). Diversity and wiring variability of olfactory local interneurons in the Drosophila antennal lobe. Nat. Neurosci. 13, 439-449.

Clyne, P., Grant, A., O'Connell, R., and Carlson, J.R. (1997). Odorant response of individual sensilla on the Drosophila antenna. Invertebr. Neurosci. IN 3, 127-135.

Clyne, P.J., Warr, C.G., Freeman, M.R., Lessing, D., Kim, J., and Carlson, J.R. (1999). A novel family of divergent seven-transmembrane proteins: candidate odorant receptors in Drosophila. Neuron 22, 327-338.

Crowson, R.A. (2013). The Biology of the Coleoptera (Academic Press).

Dacks, A.M., Green, D.S., Root, C.M., Nighorn, A.J., and Wang, J.W. (2009). Serotonin Modulates Olfactory Processing in the Antennal Lobe of Drosophila. J. Neurogenet. 23, 366-377.

Datta, S.R., Vasconcelos, M.L., Ruta, V., Luo, S., Wong, A., Demir, E., Flores, J., Balonze, K., Dickson, B.J., and Axel, R. (2008). The Drosophila pheromone cVA activates a sexually dimorphic neural circuit. Nature 452, 473-477.

De Bruyne, M., Foster, K., and Carlson, J.R. (2001). Odor Coding in the Drosophila Antenna. Neuron 30, 537-552.

Denell, R. (2008). Establishment of Tribolium as a Genetic Model System and Its Early Contributions to Evo-Devo. Genetics 180, 1779-1786.

Diehl, P.A., Vlimant, M., Guerenstein, P., and Guerin, P.M. (2003). Ultrastructure and receptor cell responses of the antennal grooved peg sensilla of Triatoma infestans (Hemiptera: Reduviidae). Arthropod Struct. Dev. 31, 271-285.

Dippel, S., Oberhofer, G., Kahnt, J., Gerischer, L., Opitz, L., Schachtner, J., Stanke, M., Schütz, S., Wimmer, E.A., and Angeli, S. (2014). Tissue-specific transcriptomics, chromosomal localization, and phylogeny of chemosensory and odorant binding proteins from the red flour beetle Tribolium castaneum reveal subgroup specificities for olfaction or more general functions. BMC Genomics 15.

Dippel, S., Kollmann, M., Oberhofer, G., Montino, A., Knoll, C., Krala, M., Rexer, K. Frank, S. , Crombach, S., Schachtner, J., Wimmer, E. (in preparation). Comprehensive 
Morphological and Transcriptomic Analysis of the Olfactory System in the Red Flour Beetle Tribolium castaneum Reveal a Novel Gnathal Olfactory Processing Center

Dönitz, J., Schmitt-Engel, C., Grossmann, D., Gerischer, L., Tech, M., Schoppmeier, M., Klingler, M., and Bucher, G. (2014). iBeetle-Base: a database for RNAi phenotypes in the red flour beetle Tribolium castaneum. Nucleic Acids Res. gku1054.

Dramane, G., Akpona, S., Besnard, P., and Khan, N.A. (2014). Cell mechanisms of gustatory lipids perception and modulation of the dietary fat preference. Biochimie 107 Pt A, 11-14.

Duehl, A.J., Arbogast, R.T., and Teal, P.E. (2011). Age and sex related responsiveness of Tribolium castaneum (Coleoptera: Tenebrionidae) in novel behavioral bioassays. Environ. Entomol. 40, 82-87.

Dweck, H.K.M., Ebrahim, S.A.M., Kromann, S., Bown, D., Hillbur, Y., Sachse, S., Hansson, B.S., and Stensmyr, M.C. (2013). Olfactory preference for egg laying on citrus substrates in Drosophila. Curr. Biol. CB 23, 2472-2480.

Febbraio, M., and Silverstein, R.L. (2007). CD36: implications in cardiovascular disease. Int. J. Biochem. Cell Biol. 39, 2012-2030.

Fleming, W.E. (1969). Attractants for the Japanese beetle (Agricultural Research Service; for sale by the Supt. of Docs., U.S. Govt. Print. Off.).

Forstner, M., Gohl, T., Breer, H., and Krieger, J. (2006). Candidate pheromone binding proteins of the silkmoth Bombyx mori. Invertebr. Neurosci. IN 6, 177-187.

Forstner, M., Gohl, T., Gondesen, I., Raming, K., Breer, H., and Krieger, J. (2008). Differential expression of SNMP-1 and SNMP-2 proteins in pheromone-sensitive hairs of moths. Chem. Senses 33, 291-299.

Franc, N.C., Heitzler, P., Ezekowitz, R.A.B., and White, K. (1999). Requirement for croquemort in phagocytosis of apoptotic cells in Drosophila. Science 284, 1991-1993.

Fushiki, T. (2014). Why fat is so preferable: from oral fat detection to inducing reward in the brain. Biosci. Biotechnol. Biochem. 78, 363-369.

Galindo, K., and Smith, D.P. (2001). A large family of divergent Drosophila odorantbinding proteins expressed in gustatory and olfactory sensilla. Genetics 159, 1059-1072.

Galizia, C.G., Sachse, S., and Mustaparta, H. (2000). Calcium responses to pheromones and plant odours in the antennal lobe of the male and female moth Heliothis virescens. J. Comp. Physiol. [A] 186, 1049-1063.

Gao, Q., and Chess, A. (1999). Identification of candidate Drosophila olfactory receptors from genomic DNA sequence. Genomics 60, 31-39. 
Gao, Q., Yuan, B., and Chess, A. (2000). Convergent projections of Drosophila olfactory neurons to specific glomeruli in the antennal lobe. Nat. Neurosci. 3, 780-785.

Gardiner, A., Barker, D., Butlin, R.K., Jordan, W.C., and Ritchie, M.G. (2008). Drosophila chemoreceptor gene evolution: selection, specialization and genome size. Mol. Ecol. 17, 1648-1657.

Getahun, M.N., Olsson, S.B., Lavista-Llanos, S., Hansson, B.S., and Wicher, D. (2013). Insect Odorant Response Sensitivity Is Tuned by Metabotropically Autoregulated Olfactory Receptors. PLoS ONE 8.

Gilles, A.F., and Averof, M. (2014). Functional genetics for all: engineered nucleases, CRISPR and the gene editing revolution. EvoDevo 5, 43.

Gnatzy, W., Mohren, W., and Steinbrecht, R.A. (1984). Pheromone receptors in Bombyx mori and Antheraea pernyi. II. Morphometric analysis. Cell Tissue Res. 235, 35-42.

Van der Goes van Naters, W., and Carlson, J.R. (2007). Receptors and Neurons for Fly Odors in Drosophila. Curr. Biol. 17, 606-612.

Gomez-Diaz, C., Reina, J.H., Cambillau, C., and Benton, R. (2013). Ligands for pheromonesensing neurons are not conformationally activated odorant binding proteins. PLoS Biol. 11, e1001546.

Greenspan, R.J. (1997). Fly Pushing: The Theory and Practice of Drosophila Genetics (Cold Spring Harbor Laboratory Press).

Grimaldi, D., and Engel, M.S. (2005). Evolution of the Insects (Cambridge University Press).

Grosse-Wilde, E., Kuebler, L.S., Bucks, S., Vogel, H., Wicher, D., and Hansson, B.S. (2011). Antennal transcriptome of Manduca sexta. Proc. Natl. Acad. Sci. U. S. A. 108, 7449-7454.

Gu, S.-H., Sun, L., Yang, R.-N., Wu, K.-M., Guo, Y.-Y., Li, X.-C., Zhou, J.-J., and Zhang, Y.-J. (2014). Molecular characterization and differential expression of olfactory genes in the antennae of the black cutworm moth Agrotis ipsilon. PloS One 9, e103420.

Gu, X.-C., Zhang, Y.-N., Kang, K., Dong, S.-L., and Zhang, L.-W. (2015). Antennal Transcriptome Analysis of Odorant Reception Genes in the Red Turpentine Beetle (RTB), Dendroctonus valens. PloS One 10, e0125159.

Guijarro, A., Fu, J., Astarita, G., and Piomelli, D. (2010). CD36 gene deletion decreases oleoylethanolamide levels in small intestine of free-feeding mice. Pharmacol. Res. Off. J. Ital. Pharmacol. Soc. 61, 27-33.

Guo, L., and Qing Li, G. (2009). Olfactory Perception of Oviposition-Deterring Fatty Acids and Their Methyl Esters by the Asian Corn Borer, Ostrinia furnacalis. J. Insect Sci. 9. 
Guven-Ozkan, T., and Davis, R.L. (2014). Functional neuroanatomy of Drosophila olfactory memory formation. Learn. Mem. Cold Spring Harb. N 21, 519-526.

Ha, T.S., and Smith, D.P. (2006). A pheromone receptor mediates 11-cis-vaccenyl acetate-induced responses in Drosophila. J. Neurosci. Off. J. Soc. Neurosci. 26, 87278733.

Hall, J.C. (1994). The mating of a fly. Science 264, 1702-1714.

Hallem, E.A., and Carlson, J.R. (2006). Coding of odors by a receptor repertoire. Cell 125, 143-160.

Hallem, E.A., Ho, M.G., and Carlson, J.R. (2004). The molecular basis of odor coding in the Drosophila antenna. Cell 117, 965-979.

Hall, T.A. (1999). BioEdit: a user-friendly biological sequence alignment editor and analysis program for Windows 95/98/NT. Nucl. Acids. Symp. Ser. 41:95-98.

Han, C., Song, Y., Xiao, H., Wang, D., Franc, N.C., Jan, L.Y., and Jan, Y.-N. (2014). Epidermal cells are the primary phagocytes in the fragmentation and clearance of degenerating dendrites in Drosophila. Neuron 81, 544-560.

Hansson, B.S., and Stensmyr, M.C. (2011). Evolution of Insect Olfaction. Neuron 72, 698711.

Hauling, T., Krautz, R., Markus, R., Volkenhoff, A., Kucerova, L., and Theopold, U. (2014). A Drosophila immune response against Ras-induced overgrowth. Biol. Open 3, 250-260.

Heimbeck, G., Bugnon, V., Gendre, N., Keller, A., and Stocker, R.F. (2001). A central neural circuit for experience-independent olfactory and courtship behavior in Drosophila melanogaster. Proc. Natl. Acad. Sci. 98, 15336-15341.

Hekmat-Scafe, D.S., Scafe, C.R., McKinney, A.J., and Tanouye, M.A. (2002). Genome-wide analysis of the odorant-binding protein gene family in Drosophila melanogaster. Genome Res. 12, 1357-1369.

Herboso, L., Talamillo, A., Pérez, C., and Barrio, R. (2011). Expression of the Scavenger Receptor Class B type I (SR-BI) family in Drosophila melanogaster. Int. J. Dev. Biol. 55, 603-611.

Hildebrand, J.G. (1996). Olfactory control of behavior in moths: central processing of odor information and the functional significance of olfactory glomeruli. J. Comp. Physiol. A 178, 5-19.

Hoebe, K., Georgel, P., Rutschmann, S., Du, X., Mudd, S., Crozat, K., Sovath, S., Shamel, L., Hartung, T., Zähringer, U., et al. (2005). CD36 is a sensor of diacylglycerides. Nature 433, 523-527. 
Hoskins, R.A., Carlson, J.W., Kennedy, C., Acevedo, D., Evans-Holm, M., Frise, E., Wan, K.H., Park, S., Mendez-Lago, M., Rossi, F., et al. (2007). Sequence finishing and mapping of Drosophila melanogaster heterochromatin. Science 316, 1625-1628.

Howard, R.W., and Blomquist, G.J. (2005). Ecological, Behavioral, and Biochemical Aspects of Insect Hydrocarbons*. Annu. Rev. Entomol. 50, 371-393.

Ignatious Raja, J.S., Katanayeva, N., Katanaev, V.L., and Galizia, C.G. (2014). Role of Go/i subgroup of $\mathrm{G}$ proteins in olfactory signaling of Drosophila melanogaster. Eur. J. Neurosci. 39, 1245-1255.

Ignell, R., Root, C.M., Birse, R.T., Wang, J.W., Nässel, D.R., and Winther, A.M. (2009). Presynaptic peptidergic modulation of olfactory receptor neurons in Drosophila. Proc. Natl. Acad. Sci. U. S. A. 106, 13070-13075.

Jin, X., Ha, T.S., and Smith, D.P. (2008). SNMP is a signaling component required for pheromone sensitivity in Drosophila. Proc. Natl. Acad. Sci. U. S. A. 105, 10996-11001.

Jones, P.L., Pask, G.M., Rinker, D.C., and Zwiebel, L.J. (2011). Functional agonism of insect odorant receptor ion channels. Proc. Natl. Acad. Sci. 108, 8821-8825.

Kaissling, K.E. (2001). Olfactory perireceptor and receptor events in moths: a kinetic model. Chem. Senses 26, 125-150.

Kartz, G.A., Holme, R.L., Nicholson, K., and Sahoo, D. (2014). SR-BI/CD36 chimeric receptors define extracellular subdomains of SR-BI critical for cholesterol transport. Biochemistry (Mosc.) 53, 6173-6182.

Kaupp, U.B. (2010). Olfactory signalling in vertebrates and insects: differences and commonalities. Nat. Rev. Neurosci. 11, 188-200.

Keil, T.A. (1997). Functional morphology of insect mechanoreceptors. Microsc. Res. Tech. 39, 506-531.

Keil, T.A. (1999). Morphology and Development of the Peripheral Olfactory Organs. In Insect Olfaction, P.D.B.S. Hansson, ed. (Springer Berlin Heidelberg), pp. 5-47.

Keil, T.A., and Steinbrecht, R.A. (1984). Mechanosensitive and Olfactory Sensilla of Insects. In Insect Ultrastructure, R.C. King, and H. Akai, eds. (Springer US), pp. 477-516.

Keilin, D. (1944). Respiratory systems and respiratory adaptations in larvae and pupae of Diptera. Parasitology 36, 1-66.

Keilin, D., Tate, P., and Vincent, M. (1935). The Perispiracular Glands of Mosquito Larvae. Parasitology 27, 257-262.

Kelley, L.A., and Sternberg, M.J.E. (2009). Protein structure prediction on the Web: a case study using the Phyre server. Nat. Protoc. 4, 363-371. 
Kelley, L.A., Mezulis, S., Yates, C.M., Wass, M.N., and Sternberg, M.J.E. (2015). The Phyre2 web portal for protein modeling, prediction and analysis. Nat. Protoc. 10, 845-858.

Kiefer, C., Sumser, E., Wernet, M.F., and Von Lintig, J. (2002). A class B scavenger receptor mediates the cellular uptake of carotenoids in Drosophila. Proc. Natl. Acad. Sci. U. S. A. 99, 10581-10586.

Kim, M.-S., and Smith, D.P. (2001). The Invertebrate Odorant-binding Protein LUSH is required for Normal Olfactory Behavior in Drosophila. Chem. Senses 26, 195-199.

Kim, H.S., Murphy, T., Xia, J., Caragea, D., Park, Y., Beeman, R.W., Lorenzen, M.D., Butcher, S., Manak, J.R., and Brown, S.J. (2010). BeetleBase in 2010: revisions to provide comprehensive genomic information for Tribolium castaneum. Nucleic Acids Res. 38, D437-D442.

Kim, J., Matsuyama, S., and Suzuki, T. (2005). 4,8-Dimethyldecanal, the Aggregation Pheromone of Tribolium castaneum, is Biosynthesized Through the Fatty Acid Pathway. J. Chem. Ecol. 31, 1381-1400.

Kleene, S.J., and Gesteland, R.C. (1991). Transmembrane currents in frog olfactory cilia. J. Membr. Biol. 120, 75-81.

Krieger, J., Grosse-Wilde, E., Gohl, T., Dewer, Y.M.E., Raming, K., and Breer, H. (2004). Genes encoding candidate pheromone receptors in a moth (Heliothis virescens). Proc. Natl. Acad. Sci. U. S. A. 101, 11845-11850.

Krieger, J., Grosse-Wilde, E., Gohl, T., and Breer, H. (2005). Candidate pheromone receptors of the silkmoth Bombyx mori. Eur. J. Neurosci. 21, 2167-2176.

Kurtovic, A., Widmer, A., and Dickson, B.J. (2007). A single class of olfactory neurons mediates behavioural responses to a Drosophila sex pheromone. Nature 446, 542-546.

Lai, W.-A., Yeh, Y.-T., Lee, M.-T., Wu, L.-S., Ke, F.-C., and Hwang, J.-J. (2013). Ovarian granulosa cells utilize scavenger receptor SR-BI to evade cellular cholesterol homeostatic control for steroid synthesis. J. Lipid Res. 54, 365-378.

Laissue, P.P., Reiter, C., Hiesinger, P.R., Halter, S., Fischbach, K.F., and Stocker, R.F. (1999). Three-dimensional reconstruction of the antennal lobe in Drosophila melanogaster. J. Comp. Neurol. 405, 543-552.

Leal, W.S. (2003). 15 - Proteins that make sense. In Insect Pheromone Biochemistry and Molecular Biology, G.B. Vogt, ed. (San Diego: Academic Press), pp. 447-476.

Leal, W.S. (2013). Odorant Reception in Insects: Roles of Receptors, Binding Proteins, and Degrading Enzymes. Annu. Rev. Entomol. 58, 373-391.

Lebreton, S., Grabe, V., Omondi, A.B., Ignell, R., Becher, P.G., Hansson, B.S., Sachse, S., and Witzgall, P. (2014). Love makes smell blind: mating suppresses pheromone attraction in Drosophila females via Or65a olfactory neurons. Sci. Rep. 4. 
Leitch, O., Papanicolaou, A., Lennard, C., Kirkbride, K.P., and Anderson, A. (2015). Chemosensory genes identified in the antennal transcriptome of the blowfly Calliphora stygia. BMC Genomics 16, 255.

Lemaitre, B. (2000). Drosophila immunity genes.

Leunissen, J. l. m., and Yi, H. (2009). Self-pressurized rapid freezing (SPRF): a novel cryofixation method for specimen preparation in electron microscopy. J. Microsc. 235, 25-35.

Li, J., Lehmann, S., Weißbecker, B., Ojeda Naharros, I., Schütz, S., Joop, G., and Wimmer, E.A. (2013). Odoriferous Defensive Stink Gland Transcriptome to Identify Novel Genes Necessary for Quinone Synthesis in the Red Flour Beetle, Tribolium castaneum. PLoS Genet. 9.

Li, Z., Ni, J.D., Huang, J., and Montell, C. (2014). Requirement for Drosophila SNMP1 for Rapid Activation and Termination of Pheromone-Induced Activity. PLoS Genet 10, e1004600.

Lindsley, D.L., and Zimm, G.G. (2012). The Genome of Drosophila melanogaster (Academic Press).

Liu, W.W., and Wilson, R.I. (2013). Glutamate is an inhibitory neurotransmitter in the Drosophila olfactory system. Proc. Natl. Acad. Sci. U. S. A. 110, 10294-10299.

Liu, S., Rao, X.-J., Li, M.-Y., Feng, M.-F., He, M.-Z., and Li, S.-G. (2015). Identification of candidate chemosensory genes in the antennal transcriptome of Tenebrio molitor (Coleoptera: Tenebrionidae). Comp. Biochem. Physiol. Part D Genomics Proteomics 13, 44-51.

Lorenzen, M.D., Brown, S.J., Denell, R.E., and Beeman, R.W. (2002). Cloning and characterization of the Tribolium castaneum eye-color genes encoding tryptophan oxygenase and kynurenine 3-monooxygenase. Genetics 160, 225-234.

Lundin, C., Käll, L., Kreher, S.A., Kapp, K., Sonnhammer, E.L., Carlson, J.R., von Heijne, G., and Nilsson, I. (2007). Membrane topology of the Drosophila OR83b odorant receptor. FEBS Lett. 581, 5601-5604.

Maddison, D.R., Schulz, K., and Maddison, W.P. The Tree of Life Web Project*.

Martin, F., and Alcorta, E. (2011). Regulation of Olfactory Transduction in the Orco Channel. Front. Cell. Neurosci. 5.

Martin, C., Chevrot, M., Poirier, H., Passilly-Degrace, P., Niot, I., and Besnard, P. (2011a). CD36 as a lipid sensor. Physiol. Behav. 105, 36-42.

Martin, J.P., Beyerlein, A., Dacks, A.M., Reisenman, C.E., Riffell, J.A., Lei, H., and Hildebrand, J.G. (2011b). The neurobiology of insect olfaction: sensory processing in a comparative context. Prog. Neurobiol. 95, 427-447. 
McKenzie, S.K., Oxley, P.R., and Kronauer, D.J.C. (2014). Comparative genomics and transcriptomics in ants provide new insights into the evolution and function of odorant binding and chemosensory proteins. BMC Genomics 15, 718.

Merritt, D.J. (1989). The cercal sensilla of the blowfly Lucilia cuprina. II. Structure of the enveloping cells and the basal regions of the sensory dendrites. Tissue Cell 21, 281-298.

Mertens, I., Meeusen, T., Huybrechts, R., De Loof, A., and Schoofs, L. (2002). Characterization of the short neuropeptide $\mathrm{F}$ receptor from Drosophila melanogaster. Biochem. Biophys. Res. Commun. 297, 1140-1148.

Meyer, A., Galizia, C.G., and Nawrot, M.P. (2013). Local interneurons and projection neurons in the antennal lobe from a spiking point of view. J. Neurophysiol. 110, 24652474.

Mikael A Carlsson, M.D. (2010). Multiple Neuropeptides in the Drosophila Antennal Lobe Suggest Complex Modulatory Circuits. J. Comp. Neurol. 518, 3359-3380.

Möller, S., Croning, M.D., and Apweiler, R. (2001). Evaluation of methods for the prediction of membrane spanning regions. Bioinforma. Oxf. Engl. 17, 646-653.

Moor, H. (1987). Theory and Practice of High Pressure Freezing. In Cryotechniques in Biological Electron Microscopy, P.D.R.A. Steinbrecht, and D.K. Zierold, eds. (Springer Berlin Heidelberg), pp. 175-191.

Mukunda, L., Lavista-Llanos, S., Hansson, B.S., and Wicher, D. (2014). Dimerisation of the Drosophila odorant coreceptor Orco. Front. Cell. Neurosci. 8.

Murao, K., Terpstra, V., Green, S.R., Kondratenko, N., Steinberg, D., and Quehenberger, O. (1997). Characterization of CLA-1, a Human Homologue of Rodent Scavenger Receptor BI, as a Receptor for High Density Lipoprotein and Apoptotic Thymocytes. J. Biol. Chem. $272,17551-17557$.

Nagai, T. (1981). Electroantennogram response gradient on the antenna of the European corn borer, Ostrinia nubilalis. J. Insect Physiol. 27, 889-894.

Nakagawa, T., and Vosshall, L.B. (2009). Controversy and Consensus: Non-Canonical Signaling Mechanisms in the Insect Olfactory System. Curr. Opin. Neurobiol. 19, 284292.

Nassir, F., Wilson, B., Han, X., Gross, R.W., and Abumrad, N.A. (2007). CD36 is important for fatty acid and cholesterol uptake by the proximal but not distal intestine. J. Biol. Chem. 282, 19493-19501.

Neuhaus, E.M., Gisselmann, G., Zhang, W., Dooley, R., Störtkuhl, K., and Hatt, H. (2005). Odorant receptor heterodimerization in the olfactory system of Drosophila melanogaster. Nat. Neurosci. 8, 15-17. 
Nichols, Z., and Vogt, R.G. (2008). The SNMP/CD36 gene family in Diptera, Hymenoptera and Coleoptera: Drosophila melanogaster, D. pseudoobscura, Anopheles gambiae, Aedes aegypti, Apis mellifera, and Tribolium castaneum. Insect Biochem. Mol. Biol. 38, 398415.

Nijland, R., and Burgess, J.G. (2010). Bacterial olfaction. Biotechnol. J. 5, 974-977.

Nixon, D.W. (1994). Chemoprevention of Cancer (CRC Press).

Obeng-Ofori, D.; T.H.C. (1990). Tribolium aggregation pheromone: monitoring, range of attraction and orientation behaviour of T. castaneum (Coleoptera: Tenebrionidae). Bull. Entomol. Res. 80, 443-451.

Olsen, S.R., and Wilson, R.I. (2008). Lateral presynaptic inhibition mediates gain control in an olfactory circuit. Nature 452, 956-960.

Pace, U., Hanski, E., Salomon, Y., and Lancet, D. (1985). Odorant-sensitive adenylate cyclase may mediate olfactory reception. Nature 316, 255-258.

Pappas, P.W., and Morrison, S.E. (1995). Benzoquinones of the beetles, Tribolium castaneum and Tribolium confusum. Prep. Biochem. 25, 155-168.

Pellegrino, M., Nakagawa, T., and Vosshall, L.B. (2010). Single sensillum recordings in the insects Drosophila melanogaster and Anopheles gambiae. J. Vis. Exp. JoVE 1-5.

Pelosi, P., and Maida, R. (1995). Odorant-binding proteins in insects. Comp. Biochem. Physiol. B Biochem. Mol. Biol. 111, 503-514.

Pelosi, P., Zhou, J.-J., Ban, L.P., and Calvello, M. (2006). Soluble proteins in insect chemical communication. Cell. Mol. Life Sci. CMLS 63, 1658-1676.

Phillips, T.W., Jiang, X.L., Burkholder, W.E., Phillips, J.K., and Tran, H.Q. (1993). Behavioral responses to food volatiles by two species of stored-product coleoptera,Sitophilus oryzae (curculionidae) andTribolium castaneum (tenebrionidae). J. Chem. Ecol. 19, 723-734.

Pifferi, S., Menini, A., and Kurahashi, T. (2010). Signal Transduction in Vertebrate Olfactory Cilia. In The Neurobiology of Olfaction, A. Menini, ed. (Boca Raton (FL): CRC Press),.

Posnien, N., Schinko, J., Grossmann, D., Shippy, T.D., Konopova, B., and Bucher, G. (2009). RNAi in the Red Flour Beetle (Tribolium). Cold Spring Harb. Protoc. 2009, pdb.prot5256.

Pregitzer, P., Schubert, M., Breer, H., Hansson, B.S., Sachse, S., and Krieger, J. (2012). Plant odorants interfere with detection of sex pheromone signals by male Heliothis virescens. Front. Cell. Neurosci. 6. 
Pregitzer, P., Greschista, M., Breer, H., and Krieger, J. (2014). The sensory neurone membrane protein SNMP1 contributes to the sensitivity of a pheromone detection system. Insect Mol. Biol. 23, 733-742.

Putri, M., Syamsunarno, M.R.A.A., Iso, T., Yamaguchi, A., Hanaoka, H., Sunaga, H., Koitabashi, N., Matsui, H., Yamazaki, C., Kameo, S., et al. (2015). CD36 is indispensable for thermogenesis under conditions of fasting and cold stress. Biochem. Biophys. Res. Commun. 457, 520-525.

Regnier, F.E., and Law, J.H. (1968). Insect pheromones. J. Lipid Res. 9, 541-551.

Richards, S., Gibbs, R.A., Weinstock, G.M., Brown, S.J., Denell, R., Beeman, R.W., Gibbs, R., Beeman, R.W., Brown, S.J., Bucher, G., et al. (2008). The genome of the model beetle and pest Tribolium castaneum. Nature 452, 949-955.

Riemensperger, T., Völler, T., Stock, P., Buchner, E., and Fiala, A. (2005). Punishment prediction by dopaminergic neurons in Drosophila. Curr. Biol. CB 15, 1953-1960.

Rizki, M.T.M. (1956). The cytophysiology of the spiracular glands of Drosophila melanogaster. J. Morphol. 98, 497-511.

Robertson, H.M., Martos, R., Sears, C.R., Todres, E.Z., Walden, K.K.O., and Nardi, J.B. (1999). Diversity of odourant binding proteins revealed by an expressed sequence tag project on male Manduca sexta moth antennae. Insect Mol. Biol. 8, 501-518.

Roelofs, W., Comeau, A., Hill, A., and Milicevic, G. (1971). Sex attractant of the codling moth: characterization with electroantennogram technique. Science 174, 297-299.

Rogers, M.E., Sun, M., Lerner, M.R., and Vogt, R.G. (1997). Snmp-1, a novel membrane protein of olfactory neurons of the silk moth Antheraea polyphemus with homology to the CD36 family of membrane proteins. J. Biol. Chem. 272, 14792-14799.

Rogers, M.E., Krieger, J., and Vogt, R.G. (2001). Antennal SNMPs (sensory neuron membrane proteins) of lepidoptera define a unique family of invertebrate CD36-like proteins. J. Neurobiol. 49, 47-61.

Ronderos, D.S., Lin, C.-C., Potter, C.J., and Smith, D.P. (2014). Farnesol-detecting olfactory neurons in Drosophila. J. Neurosci. Off. J. Soc. Neurosci. 34, 3959-3968.

Root, C.M., Masuyama, K., Green, D.S., Enell, L.E., Nässel, D.R., Lee, C.-H., and Wang, J.W. (2008). A Presynaptic Gain Control Mechanism Fine-Tunes Olfactory Behavior. Neuron $59,311-321$.

Roth, L.M., and Willis, E.R. (1951). Hygroreceptors in adults of tribolium (coleoptera, tenebrionidae). J. Exp. Zool. 116, 527-570.

Rowat, A.C., Keller, D., and Ipsen, J.H. (2005). Effects of farnesol on the physical properties of DMPC membranes. Biochim. Biophys. Acta BBA - Biomembr. 1713, 29-39. 
Sachse, S., and Galizia, C.G. (2002). Role of Inhibition for Temporal and Spatial Odor Representation in Olfactory Output Neurons: A Calcium Imaging Study. J. Neurophysiol. 87, 1106-1117.

Sachse, S., Rappert, A., and Galizia, C.G. (1999). The spatial representation of chemical structures in the antennal lobe of honeybees: steps towards the olfactory code. Eur. J. Neurosci. 11, 3970-3982.

Sachse, S., Peele, P., Silbering, A.F., Gühmann, M., and Galizia, C.G. (2006). Role of histamine as a putative inhibitory transmitter in the honeybee antennal lobe. Front. Zool. 3, 22.

Saitou, N., and Nei, M. (1987). The neighbor-joining method: a new method for reconstructing phylogenetic trees. Mol. Biol. Evol. 4, 406-425.

Sakudoh, T., Kuwazaki, S., Iizuka, T., Narukawa, J., Yamamoto, K., Uchino, K., Sezutsu, H., Banno, Y., and Tsuchida, K. (2013). CD36 homolog divergence is responsible for the selectivity of carotenoid species migration to the silk gland of the silkworm Bombyx mori. J. Lipid Res. 54, 482-495.

Sakurai, T., Namiki, S., and Kanzaki, R. (2014). Molecular and neural mechanisms of sex pheromone reception and processing in the silkmoth Bombyx mori. Integr. Physiol. 5, 125.

Sambrook, J., and Russell, D.W. (2001). Molecular Cloning: A Laboratory Manual (CSHL Press).

Sanger, F., and Coulson, A.R. (1975). A rapid method for determining sequences in DNA by primed synthesis with DNA polymerase. J. Mol. Biol. 94, 441-448.

Sato, K., Pellegrino, M., Nakagawa, T., Nakagawa, T., Vosshall, L.B., and Touhara, K. (2008). Insect olfactory receptors are heteromeric ligand-gated ion channels. Nature $452,1002-1006$.

Schachtner, J. M.S. (2005). Organization and evolutionary trends of primary olfactory brain centers in Tetraconata (Crustacea Hexapoda). Arthropod Struct. Amp Dev. ARTHROPOD STRUCT DEV 34, 257-299.

Schindelin, J., Arganda-Carreras, I., Frise, E., Kaynig, V., Longair, M., Pietzsch, T., Preibisch, S., Rueden, C., Saalfeld, S., Schmid, B., et al. (2012). Fiji: an open-source platform for biological-image analysis. Nat. Methods 9, 676-682.

Schinko, J.B., Weber, M., Viktorinova, I., Kiupakis, A., Averof, M., Klingler, M., Wimmer, E.A., and Bucher, G. (2010). Functionality of the GAL4/UAS system in Tribolium requires the use of endogenous core promoters. BMC Dev. Biol. 10, 53.

Schinko, J.B., Hillebrand, K., and Bucher, G. (2012). Heat shock-mediated misexpression of genes in the beetle Tribolium castaneum. Dev. Genes Evol. 222, 287-298. 
Schlyter, F., Birgersson, G., Byers, J.A., Löfqvist, J., and Bergström, G. (1987). Field response of spruce bark beetle,Ips typographus, to aggregation pheromone candidates. J. Chem. Ecol. 13, 701-716.

Schmidt, J.O. (1994). Attraction of reproductive honey bee swarms to artificial nests by Nasonov pheromone. J. Chem. Ecol. 20, 1053-1056.

Schneider, I. (1972). Cell lines derived from late embryonic stages of Drosophila melanogaster. J. Embryol. Exp. Morphol. 27, 353-365.

Schneider, H., Staudacher, S., Poppelreuther, M., Stremmel, W., Ehehalt, R., and Füllekrug, J. (2014). Protein mediated fatty acid uptake: synergy between CD36/FAT-facilitated transport and acyl-CoA synthetase-driven metabolism. Arch. Biochem. Biophys. 546, 818.

Schultze, A., Schymura, D., Forstner, M., and Krieger, J. (2012). Expression pattern of a "Plus-C" class odorant binding protein in the antenna of the malaria vector Anopheles gambiae. Insect Mol. Biol. 21, 187-195.

Schymura, D., Forstner, M., Schultze, A., Kröber, T., Swevers, L., Iatrou, K., and Krieger, J. (2010). Antennal expression pattern of two olfactory receptors and an odorant binding protein implicated in host odor detection by the malaria vector Anopheles gambiae. Int. J. Biol. Sci. 6, 614-626.

Seki, Y., Rybak, J., Wicher, D., Sachse, S., and Hansson, B.S. (2010). Physiological and morphological characterization of local interneurons in the Drosophila antennal lobe. J. Neurophysiol. 104, 1007-1019.

Shanbhag, S.R., Müller, B., and Steinbrecht, R.A. (2000). Atlas of olfactory organs of Drosophila melanogaster 2. Internal organization and cellular architecture of olfactory sensilla. Arthropod Struct. Dev. 29, 211-229.

Shields, V.D.C. (2008). Ultrastructure of Insect Sensilla. In Encyclopedia of Entomology, J.L. Capinera, ed. (Springer Netherlands), pp. 4009-4023.

Shukle, R.H. (2008). Hessian Fly, Mayetiola destructor (Say) (Diptera: Cecidomyiidae). In Encyclopedia of Entomology, J.L. Capinera, ed. (Springer Netherlands), pp. 1794-1797.

Silbering, A.F., Rytz, R., Grosjean, Y., Abuin, L., Ramdya, P., Jefferis, G.S.X.E., and Benton, R. (2011). Complementary function and integrated wiring of the evolutionarily distinct Drosophila olfactory subsystems. J. Neurosci. Off. J. Soc. Neurosci. 31, 13357-13375.

Sklar, P.B., Anholt, R.R., and Snyder, S.H. (1986). The odorant-sensitive adenylate cyclase of olfactory receptor cells. Differential stimulation by distinct classes of odorants. J. Biol. Chem. 261, 15538-15543.

Smart, R., Kiely, A., Beale, M., Vargas, E., Carraher, C., Kralicek, A.V., Christie, D.L., Chen, C., Newcomb, R.D., and Warr, C.G. (2008). Drosophila odorant receptors are novel seven 
transmembrane domain proteins that can signal independently of heterotrimeric $G$ proteins. Insect Biochem. Mol. Biol. 38, 770-780.

Smith, C.R., Smith, C.D., Robertson, H.M., Helmkampf, M., Zimin, A., Yandell, M., Holt, C., Hu, H., Abouheif, E., Benton, R., et al. (2011). Draft genome of the red harvester ant Pogonomyrmex barbatus. Proc. Natl. Acad. Sci. U. S. A. 108, 5667-5672.

Song, G.J., Kim, S.-M., Park, K.-H., Kim, J., Choi, I., and Cho, K.-H. (2015). SR-BI mediates high density lipoprotein (HDL)-induced anti-inflammatory effect in macrophages. Biochem. Biophys. Res. Commun. 457, 112-118.

Steinbrecht, R.A. (1996). Structure and function of insect olfactory sensilla. Ciba Found. Symp. 200, 158-174; discussion 174-177.

Steinbrecht, R.A., and Gnatzy, W. (1984). Pheromone receptors in Bombyx mori and Antheraea pernyi. I. Reconstruction of the cellular organization of the sensilla trichodea. Cell Tissue Res. 235, 25-34.

Steinbrecht, R.A., Ozaki, M., and Ziegelberger, G. (1992). Immunocytochemical localization of pheromone-binding protein in moth antennae. Cell Tissue Res. 270, 287302.

Stengl, M. (2010). Pheromone transduction in moths. Front. Cell. Neurosci. 4, 133.

Stengl, M., and Funk, N.W. (2013). The role of the coreceptor Orco in insect olfactory transduction. J. Comp. Physiol. A 199, 897-909.

Stensmyr, M.C., Dweck, H.K.M., Farhan, A., Ibba, I., Strutz, A., Mukunda, L., Linz, J., Grabe, V., Steck, K., Lavista-Llanos, S., et al. (2012). A Conserved Dedicated Olfactory Circuit for Detecting Harmful Microbes in Drosophila. Cell 151, 1345-1357.

Stewart, C.R., Stuart, L.M., Wilkinson, K., van Gils, J.M., Deng, J., Halle, A., Rayner, K.J., Boyer, L., Zhong, R., Frazier, W.A., et al. (2010). CD36 ligands promote sterile inflammation through assembly of a Toll-like receptor 4 and 6 heterodimer. Nat. Immunol. 11, 155-161.

Strutz, A., Soelter, J., Baschwitz, A., Farhan, A., Grabe, V., Rybak, J., Knaden, M., Schmuker, M., Hansson, B.S., and Sachse, S. (2014). Decoding odor quality and intensity in the Drosophila brain. eLife 3, e04147.

Stuart, L.M., Deng, J., Silver, J.M., Takahashi, K., Tseng, A.A., Hennessy, E.J., Ezekowitz, R.A.B., and Moore, K.J. (2005). Response to Staphylococcus aureus requires CD36mediated phagocytosis triggered by the $\mathrm{COOH}$-terminal cytoplasmic domain. J. Cell Biol. 170, 477-485.

Subaharan, K., Kumar, A.R.V., and Ganiger, P. (2013). Electrophysiological responses of chafer beetle, Holotrichia serrata (F.) (Coleoptera: Scarabaeidae). J. Saudi Soc. Agric. Sci. $12,155-159$. 
Sun, M., Finnemann, S.C., Febbraio, M., Shan, L., Annangudi, S.P., Podrez, E.A., Hoppe, G., Darrow, R., Organisciak, D.T., Salomon, R.G., et al. (2006). Light-induced oxidation of photoreceptor outer segment phospholipids generates ligands for CD36-mediated phagocytosis by retinal pigment epithelium: a potential mechanism for modulating outer segment phagocytosis under oxidant stress conditions. J. Biol. Chem. 281, 42224230.

Suzuki, T. (1980). 4,8-Dimethyldecanal: The Aggregation Pheromone of the Flour Beetles, Tribolium castaneum and T. confusum (Coleoptera: Tenebrionidae). Agric. Biol. Chem. 44, 2519-2520.

Tamura, K., Peterson, D., Peterson, N., Stecher, G., Nei, M., and Kumar, S. (2011). MEGA5: molecular evolutionary genetics analysis using maximum likelihood, evolutionary distance, and maximum parsimony methods. Mol. Biol. Evol. 28, 2731-2739.

Tanaka, N.K., Endo, K., and Ito, K. (2012). Organization of antennal lobe-associated neurons in adult Drosophila melanogaster brain. J. Comp. Neurol. 520, 4067-4130.

Tegoni, M., Campanacci, V., and Cambillau, C. (2004). Structural aspects of sexual attraction and chemical communication in insects. Trends Biochem. Sci. 29, 257-264.

Thompson, J.D., Higgins, D.G., and Gibson, T.J. (1994). CLUSTAL W: improving the sensitivity of progressive multiple sequence alignment through sequence weighting, position-specific gap penalties and weight matrix choice. Nucleic Acids Res. 22, 46734680.

Thurm, U., and Küppers, J. (1980). EPITHELIAL PHYSIOLOGY OF INSECT SENSILLA. In Insect Biology in the Future, M.L.S. Smith, ed. (Academic Press), pp. 735-763.

Tomoyasu, Y., and Denell, R.E. (2004). Larval RNAi in Tribolium (Coleoptera) for analyzing adult development. Dev. Genes Evol. 214, 575-578.

Touhara, K. (2013). Electroantennogram and Single Sensillum Recording in Insect Antennae - Springer (Humana Press),.

Unruh, L.M., Xu, R., and Kramer, K.J. (1998). Benzoquinone levels as a function of age and gender of the red flour beetle, Tribolium castaneum. Insect Biochem. Mol. Biol. 28, 969977.

Venkatesh, S., and Naresh Singh, R. (1984). Sensilla on the third antennal segment of Drosophila melanogaster meigen (Diptera: Drosophilidae). Int. J. Insect Morphol. Embryol. 13, 51-63.

Vieira, F.G., and Rozas, J. (2011). Comparative Genomics of the Odorant-Binding and Chemosensory Protein Gene Families across the Arthropoda: Origin and Evolutionary History of the Chemosensory System. Genome Biol. Evol. 3, 476-490.

Vogt, R.G. Biochemical diversity of odor detection: OBPs, ODEs and SNMPs. 
Vogt, R.G., and Riddiford, L.M. (1981). Pheromone binding and inactivation by moth antennae. Nature 293, 161-163.

Vogt, R.G., Rogers, M.E., Franco, M., and Sun, M. (2002). A comparative study of odorant binding protein genes: differential expression of the PBP1-GOBP2 gene cluster in Manduca sexta (Lepidoptera) and the organization of OBP genes in Drosophila melanogaster (Diptera). J. Exp. Biol. 205, 719-744.

Vogt, R.G., Miller, N.E., Litvack, R., Fandino, R.A., Sparks, J., Staples, J., Friedman, R., and Dickens, J.C. (2009). The insect SNMP gene family. Insect Biochem. Mol. Biol. 39, 448456.

Voolstra, O., Kiefer, C., Hoehne, M., Welsch, R., Vogt, K., and von Lintig, J. (2006). The Drosophila class B scavenger receptor NinaD-I is a cell surface receptor mediating carotenoid transport for visual chromophore synthesis. Biochemistry (Mosc.) 45, 13429-13437.

Vosshall, L.B. (2000). Olfaction in Drosophila. Curr. Opin. Neurobiol. 10, 498-503.

Vosshall, L.B., and Hansson, B.S. (2011). A unified nomenclature system for the insect olfactory coreceptor. Chem. Senses 36, 497-498.

Vosshall, L.B., Amrein, H., Morozov, P.S., Rzhetsky, A., and Axel, R. (1999). A Spatial Map of Olfactory Receptor Expression in the Drosophila Antenna. Cell 96, 725-736.

Wang, P. (2009). Functional Evolution of Odorants Binding Protein Genes in Drosophila Melanogaster (ProQuest).

Wang, G., Carey, A.F., Carlson, J.R., and Zwiebel, L.J. (2010). Molecular basis of odor coding in the malaria vector mosquito Anopheles gambiae. Proc. Natl. Acad. Sci. U. S. A. $107,4418-4423$.

Wang, J., Li, D.-Z., Min, S.-F., Mi, F., Zhou, S.-S., and Wang, M.-Q. (2014). Analysis of chemosensory gene families in the beetle Monochamus alternatus and its parasitoid Dastarcus helophoroides. Comp. Biochem. Physiol. Part D Genomics Proteomics 11, 1-8.

Wang, L., Wang, S., Li, Y., Paradesi, M.S.R., and Brown, S.J. (2007a). BeetleBase: the model organism database for Tribolium castaneum. Nucleic Acids Res. 35, D476-D479.

Wang, T., Jiao, Y., and Montell, C. (2007b). Dissection of the pathway required for generation of vitamin A and for Drosophila phototransduction. J. Cell Biol. 177, 305-316.

White, G.G., and Lambkin, T.A. (1988). Damage to wheat grain by larvae of Tribolium castaneum (Herbst) (Coleoptera: Tenebrionidae). J. Stored Prod. Res. 24, 61-65.

Wibe, A. (2004). How the choice of method influence on the results in electrophysiological studies of insect olfaction. J. Insect Physiol. 50, 497-503. 
Wicher, D., Schäfer, R., Bauernfeind, R., Stensmyr, M.C., Heller, R., Heinemann, S.H., and Hansson, B.S. (2008). Drosophila odorant receptors are both ligand-gated and cyclicnucleotide-activated cation channels. Nature 452, 1007-1011.

Wicher, D., Schäfer, R., Bauernfeind, R., Stensmyr, M.C., Heller, R., Heinemann, S.H., and Hansson, B.S. (2009). dOr83b--receptor or ion channel? Ann. N. Y. Acad. Sci. 1170, 164167.

Williams, I.H., Pickett, J.A., and Martin, A.P. (1981). The Nasonov pheromone of the honeybeeApis mellifera L. (Hymenoptera, Apidae). Part II. Bioassay of the components using foragers. J. Chem. Ecol. 7, 225-237.

Wilson, R.I. (2013). Early Olfactory Processing in Drosophila: Mechanisms and Principles. Annu. Rev. Neurosci. 36, 217-241.

Wilson, R.I., and Laurent, G. (2005). Role of GABAergic inhibition in shaping odorevoked spatiotemporal patterns in the Drosophila antennal lobe. J. Neurosci. Off. J. Soc. Neurosci. 25, 9069-9079.

Wojtasek, H., and Leal, W.S. (1999). Conformational change in the pheromone-binding protein from Bombyx mori induced by $\mathrm{pH}$ and by interaction with membranes. J. Biol. Chem. 274, 30950-30956.

Yao, C.A., Ignell, R., and Carlson, J.R. (2005). Chemosensory coding by neurons in the coeloconic sensilla of the Drosophila antenna. J. Neurosci. Off. J. Soc. Neurosci. 25, 83598367.

Zarzo, M. (2007). The sense of smell: molecular basis of odorant recognition. Biol. Rev. $82,455-479$.

Zettler, L.J., and Cuperus, G.W. (1990). Pesticide Resistance in Tribolium castaneum (Coleoptera: Tenebrionidae) and Rhyzopertha dominica (Coleoptera: Bostrichidae) in Wheat. J. Econ. Entomol. 83, 1677-1681.

Zhang, L., Clarke, S.R., and Sun, J. (2009). Electrophysiological and behavioral responses of Dendroctonus valens (Coleoptera: Curculionidae: Scolytinae) to four bark beetle pheromones. Environ. Entomol. 38, 472-477.

Zhang, S., Zhang, Z., Wang, H., and Kong, X. (2014a). Antennal transcriptome analysis and comparison of olfactory genes in two sympatric defoliators, Dendrolimus houi and Dendrolimus kikuchii (Lepidoptera: Lasiocampidae). Insect Biochem. Mol. Biol. 52, 6981.

Zhang, Y., Zheng, Y., Li, D., and Fan, Y. (2014b). Transcriptomics and identification of the chemoreceptor superfamily of the pupal parasitoid of the oriental fruit fly, Spalangia endius Walker (Hymenoptera: Pteromalidae). PloS One 9, e87800. 


\section{Acknowledgement}

Als erstes bedanke ich mich bei Prof. Ernst Wimmer für die Möglichkeit meine Doktorarbeit an seinem Institut $\mathrm{zu}$ bearbeiten und für die Bereitstellung des Arbeitsplatzes sowie des nötigen Laborzubehörs. Außerdem möchte ich mich dafür bedanken, dass er beratend an meiner Seite stand, wenn es wirklich nötig war.

Des Weiteren gilt mein Dank den Mitgliedern meines thesis committees, Prof. André Fiala und Prof. Martin Göpfert. Danke für die angenehme und inspirierende Atmosphäre bei den jährlichen Treffen. An dieser Stelle möchte ich ein besonderes Dankeschön an Prof. André Fiala richten, der mir erlaubte, an seinem Institut Teile dieser Arbeit zu verwirklichen und dabei als kompetenter Ansprechpartner immer zur Verfügung stand. Vielen Dank!

Bedanken möchte ich mich auch bei der ganzen entwicklungsbiologischen Abteilung. Danke für die freundliche und hilfsbereite Arbeitsatmosphäre. Danke an Stefan Dippel für die Einweisung in die Methoden, die vor allem zu Beginn meiner Promotionszeit wichtig waren und für viele inspirierende Gespräche. Ein besonderes Dankeschön geht an Elke Küster für die Hilfe bei der Fliegen- als auch Käferarbeit. Durch Dich wurde meine Frustrationstoleranz immer gesteigert. Danke an die 13:00 Uhr Mensagruppe, bestehend aus Ingrid Curril, Jianwei Li, Kefei Yang, Christian Ogaugwu und Montse Torres für entspannende Mittagspausen. Ein großes Dankeschön an Sabrina Lehmann und Janna Siemanowski dafür, dass ihr immer für Gespräche und Aktivitäten im, als auch außerhalb des Labors da wart und dadurch zu mehr als nur Kolleginnen geworden seid.

Vielen Dank auch an alle Mitglieder der Abteilung „Molekulare Neurobiologie des Verhaltens" für die angenehme Arbeitsatmosphäre in Eurer Abteilung. Ein ganz 
Acknowledgement

besonderes Dankeschön geht hierbei an Ulrike Pech für die Einweisung und die Unterstützung beim abendlichen imagen.

Danke an Karthi Balakrishnan für die Durchführung der EAGs.

Vielen Dank an Bernhard Schmid für die gemeinsam verbrachten Kaffeepausen, die sowohl gefüllt waren mit wissenschaftlichen Diskussionen als auch mit leichterer Kost. Vielen Dank für die Unterstützung während meiner gesamten Promotionszeit und die besondere Unterstützung in den letzten Wochen. Ich bin froh, dass sich zwischen uns eine so tolle Freundschaft entwickelt hat. Welcome to Tabata!

Mein größter Dank gilt meinem Ehemann Marco Montino. Die letzten Jahre der gemeinsamen Promotion waren eine aufregende Zeit, die deutlich gemacht hat, dass wir gemeinsam alles schaffen. Vielen Dank für Deine stetige Unterstützung!

Als letztes möchte ich meiner Oma Christine Kreissig danken, die meinen gegangenen Lebensweg und mein Studium erst ermöglich hat. Danke dass Du immer an mich geglaubt hast! Ich bin froh, dass es Dich gibt! 


\section{Curriculum vitae}

\section{Personal Data}

\begin{tabular}{l|l} 
Name & $\begin{array}{l}\text { Alice Christine Montino, née Metzger } \\
\text { Born }\end{array}$ \\
Marital status & $\begin{array}{l}\text { married } \\
\text { mürtingen a. N. (Baden Württemberg), Germany }\end{array}$
\end{tabular}

\section{Education}

\section{1/2012 - $\quad$ Dissertation at the Georg-August-University of Göttingen}

present $\quad$ Thesis Title: "Cellular mechanisms of odor detection in the olfactory system of the red flour beetle Tribolium castaneum" GGNB program: "Sensory and Motor Neuroscience"

Department of Developmental Biology, Johann-Friedrich Blumenbach Institute of Zoology and Anthropology, Prof. Dr. Ernst A. Wimmer

$10 / 2006-$ Studies in Biology at the University of Hohenheim (Stuttgart,
10/2011 Germany) - Diploma Major subjects: Physiology

Minor subjects: Biochemistry and Membrane Physiology

Diploma thesis: University of Hohenheim, Stuttgart, Department of Physiology, Prof. Dr. H. Breer "Charakterisierung molekularer Elemente des olfaktorischen Systems der Malariamücke Anopheles gambiae"

Diploma Grade: "sehr gut" (1.1)

\begin{tabular}{l|l}
\hline $2003-2005$ & $\begin{array}{l}\text { Apprenticeship: Veterinary Nurse } \\
\text { Dr. F. Merkt, Esslingen a. N., Germany }\end{array}$ \\
\hline $1994-2002$ & Gymnasium Neckartenzlingen, Germany (Abitur) \\
$1993-1994$ & Merzschule Stuttgart, Germany (Gymnasium) \\
$1989-1993$ & Merzschule Stuttgart, Germany (Grundschule)
\end{tabular}

